\title{
A Metal-Free Three-Component Domino Approach to Phosphonylated Triazolines and Triazoles
}

\author{
Shakir Ahamad, Ruchir Kant and Kishor Mohanan*
}

Medicinal and Process Chemistry Division,

CSIR-Central Drug Research Institute, Jankipuram Extension, Sitapur road,

Lucknow, India-226031

kishor.mohanan@cdri.res.in

\section{SUPPORTING INFORMATION}

Content:

1. General experimental information (p. S2)

2. Optimization studies (p. S3)

3. General Procedure (p. S6)

4. Characterization data for compounds $4 a-4 a g$ \& $6 a-6 m(p .56)$

5. Copies of ${ }^{1} \mathrm{H},{ }^{13} \mathrm{C}$ and ${ }^{31} \mathrm{P}$ NMR spectra for all compounds (p. S26)

6. Copies of HRMS spectra for all compounds (p. S95) 


\section{General experimental information:}

Unless otherwise specified, all reactions were carried out under air atmosphere in ovendried round-bottom flasks. Dimethyl 2-oxopropylphosphonate, aldehydes and amines were purchased from commercial sources and were used without further purification. The reactions were monitored by TLC visualized by UV $(254 \mathrm{~nm})$ and/or with iodine. Flash chromatography was performed on 100-200 mesh silica gel using the gradient system ethylacetate-hexane (0-50\%)/ Acetone-Dichloromethane (0-30\%). NMR data were recorded at Bruker AV $400 \mathrm{MHz}$ in $\mathrm{CDCl}_{3}$ using as internal standards the residual $\mathrm{CHCl}_{3}$ signal for ${ }^{1} \mathrm{H}$ $\operatorname{NMR}(\delta=7.26 \mathrm{ppm})$ and the deuterated solvent signal for ${ }^{13} \mathrm{C} \operatorname{NMR}(\delta=77.16 \mathrm{ppm})$. Coupling constants are given in $\operatorname{Hertz}(\mathrm{Hz})$ and the classical abbreviations are used to describe the signal multiplicities. Melting points were measured with a Büchi B-540 apparatus and are uncorrected. High resolution mass spectra were obtained using Q-TOF mass spectrometer. All commercially available reagents were used as received.

The Bestmann-Ohira reagent was synthesized from the corresponding Dimethyl 2oxopropylphosphonate using a known procedure. ${ }^{1}$

(1) Callant, P.; Dhaenens,L.; Vandewalle, M. synth. Commun. 1984, 14, 155 


\section{Optimization details for 1,2,3-triazoline synthesis}

Table S1: Optimization by varying stochiometry of BOR and propyl amine

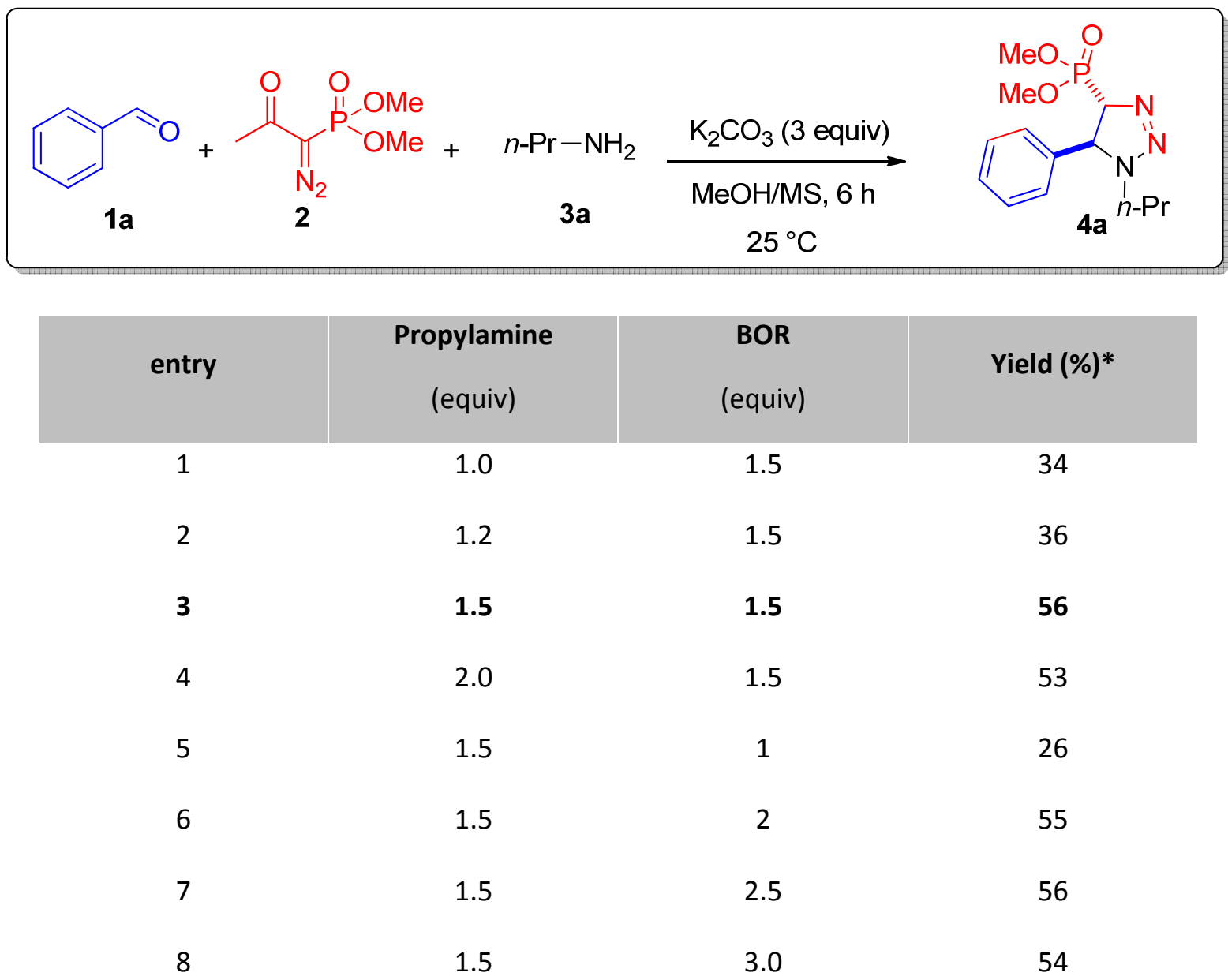

${ }^{*}{ }^{1} \mathrm{H}$ NMR yield using $\mathrm{CH}_{2} \mathrm{Br}_{2}$ as internal standard. 
Table S2: Optimization by varying bases

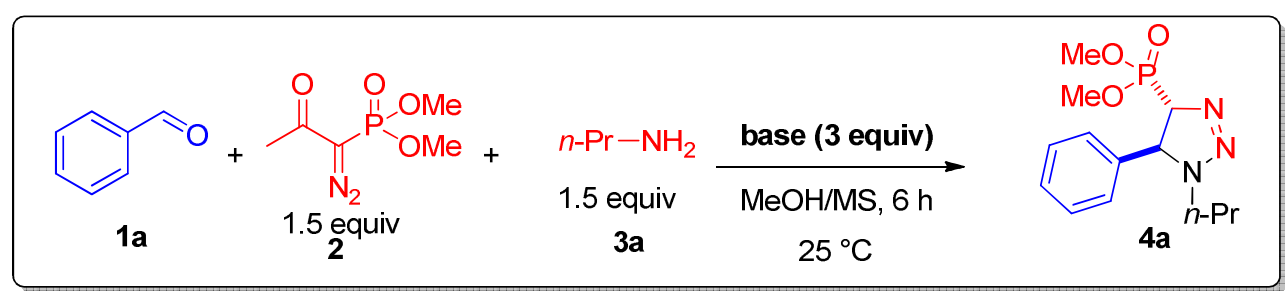

$\begin{array}{cccc}\text { entry } & \text { base } & \text { Yield (\%)* } \\ 1 & \mathrm{NaOH} & 17 \\ 2 & \mathrm{~K}^{\mathrm{t}}-\mathrm{OBu} & 23 \\ 3 & \mathrm{Et}_{3} \mathrm{~N} & 81 \\ 4 & \mathrm{DBU} & 41 \\ 5 & \mathrm{KOH} & 27 \\ 6 & \mathrm{NaOMe} & 30 \\ 7 & \mathrm{Cs}_{2} \mathrm{CO}_{3} & 55 \\ 8 & \mathrm{~K}_{2} \mathrm{CO}_{3} & 56 \\ * 9 & \mathrm{Et}_{3} \mathrm{~N}(1.5 \text { equiv }) & 82 \\ 10 & - & 82\end{array}$

$*{ }^{1} \mathrm{H}$ NMR yield using $\mathrm{CH}_{2} \mathrm{Br}_{2}$ as internal standard. 
Table S3: Optimization by varying solvents and drying agents

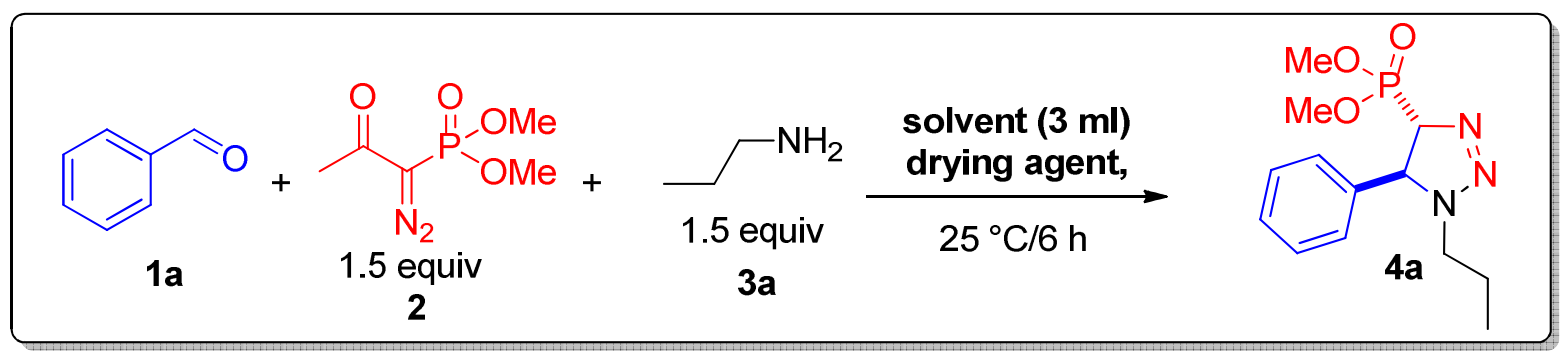

\begin{tabular}{c|c|c|c|}
\hline Entry & solvent & Drying agent & Yield (\%) \\
\hline 1 & $\mathrm{MeOH}$ & $\mathrm{MS} 4 \AA$ & 84 \\
2 & $\mathrm{MeOH}$ & $\mathrm{Na}_{2} \mathrm{SO}_{4}$ & 50 \\
3 & $\mathrm{MeOH}$ & $\mathrm{MgSO}_{4}$ & 41 \\
4 & $\mathrm{MeOH}$ & - & 83 \\
5 & $\mathrm{EtOH}$ & - & 65 \\
\hline
\end{tabular}

$*{ }^{1} \mathrm{H}$ NMR yield using $\mathrm{CH}_{2} \mathrm{Br}_{2}$ as internal standard.

\section{Procedures}

General procedure for the synthesis of phosphonyltriazolines 4

To an oven-dried round bottom flask was added benzaldehyde $1 \mathrm{a}$ (50 $\mathrm{mg}, 0.47 \mathrm{mmol}$ ), propyl amine 3a $(42 \mathrm{mg}, 0.71 \mathrm{mmol}$ ) and Bestmann-Ohira reagent 2 (136 $\mathrm{mg}, 0.71 \mathrm{mmol}$ ) and dissolved in $3 \mathrm{ml}$ of $\mathrm{MeOH}$. The reaction mixture was stirred at $25{ }^{\circ} \mathrm{C}$ for the specified time. After the completion of reaction, as indicated by TLC, solvent was evaporated off and extracted using ethyl acetate. The organic layer was dried over $\mathrm{Na}_{2} \mathrm{SO}_{4}$ and evaporated under reduced pressure. The residue was purified using column chromatography (100-200 mesh silica gel) using ethylacetate /hexane or Acetone/Dichloromethane as the eluent to afford the product 4 . 


\section{Characterization data of triazoline 4a-4ag}

Compound 4a: Dimethyl (5-phenyl-1-propyl-4,5-dihydro-1H-1,2,3-triazol-4-yl)phosphonate

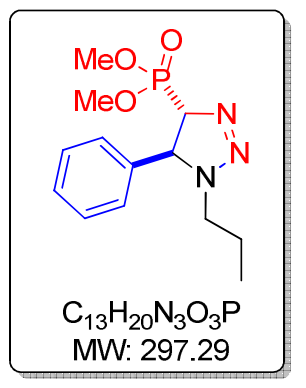

Following the general procedure, treatment of benzaldehyde $1 \mathrm{a}$ (50 mg, $0.47 \mathrm{mmol}$ ) with $\mathrm{n}$-propylamine $3 \mathrm{3a}(42 \mathrm{mg}, 0.71 \mathrm{mmol})$ and BOR (136 $\mathrm{mg}, 0.71 \mathrm{mmol}$ ) in $\mathrm{MeOH}(3 \mathrm{ml})$ at room temperature for $6 \mathrm{~h}$ followed by column chromatography afforded the product $4 \mathbf{a}$ as a colorless liquid (112 mg, 80\%). $\mathbf{R}_{\mathbf{f}}$ (Ethyl acetate/Hexane : 50/50) = 0.38. ${ }^{13} \mathbf{C}$ NMR (100 $\left.\mathrm{MHz}, \delta \mathrm{ppm} / \mathrm{CDCl}_{3}\right): 138.9\left(\mathrm{~d}, J_{C-P}=9.8 \mathrm{~Hz}, \mathrm{C}\right), 129.1(\mathrm{CH}), 129.1(\mathrm{CH})$, $128.8(\mathrm{CH}), 127.0(\mathrm{CH}), 127.0(\mathrm{CH}), 81.8\left(\mathrm{~d}, J_{C-P}=159.1 \mathrm{~Hz}, \mathrm{CH}\right), 62.5(\mathrm{CH})$, $53.9\left(\mathrm{~d}, J_{C-P}=6.5 \mathrm{~Hz}, \mathrm{CH}_{3}\right), 53.4\left(\mathrm{~d}, J_{\mathrm{C}-\mathrm{P}}=6.8 \mathrm{~Hz}, \mathrm{CH}_{3}\right), 49.7\left(\mathrm{CH}_{2}\right), 21.1\left(\mathrm{CH}_{2}\right), 11.2\left(\mathrm{CH}_{3}\right) .{ }^{1} \mathrm{H}$ NMR (400 MHz, $\left.\delta p p m / \mathrm{CDCl}_{3}\right): 7.35-7.29(\mathrm{~m}, 3 \mathrm{H}), 7.20-7.18(\mathrm{~m}, 2 \mathrm{H}), 4.68(\mathrm{dd}, J=26.0,10.8$ $\mathrm{Hz}, 1 \mathrm{H}$ ), 4.53 (dd, $J=17.2,11.2 \mathrm{~Hz}, 1 \mathrm{H}), 3.82(\mathrm{~d}, J=11.2 \mathrm{~Hz}, 3 \mathrm{H}), 3.75(\mathrm{~d}, J=10.8 \mathrm{~Hz}, 3 \mathrm{H})$, 3.60-3.56 (m, $1 \mathrm{H}), 3.29-3.22(\mathrm{~m}, 1 \mathrm{H}), 1.64-1.55(\mathrm{~m}, 2 \mathrm{H}), 0.87(\mathrm{t}, J=7.6 \mathrm{~Hz}, 3 \mathrm{H}) .{ }^{31} \mathbf{P}$ NMR (161.9 MHz, $\mathrm{CDCl}_{3}$ ): 20.89. HRMS for $\mathrm{C}_{13} \mathrm{H}_{20} \mathrm{~N}_{3} \mathrm{NaO}_{3} \mathrm{P}^{+}$: calcd. [M+Na] ${ }^{+}: 320.1134$, found: 320.1130. FTIR ( $\left.\mathrm{cm}^{-1}\right)$ : 3401, 3020, 2400, 1712, 1626, 1493, 1403, 1216, 1052, 929, 768, 669, $627,557$.

Compound 4b: Dimethyl (5-(4-methoxyphenyl)-1-propyl-4,5-dihydro-1H-1,2,3-triazol-4yl)phosphonate

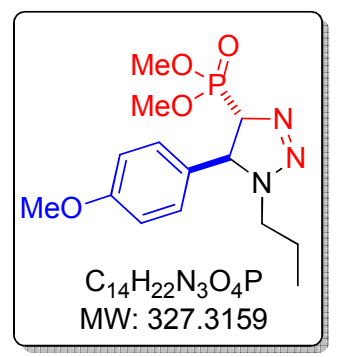

Following the general procedure, treatment of 4-methoxy benzaldehyde $\mathbf{1 b}$ (50 $\mathrm{mg}, 0.37 \mathrm{mmol}$ ) with n-propylamine $3 \mathbf{3}$ (33 $\mathrm{mg}$, $0.55 \mathrm{mmol}$ ) and BOR (106 mg, $0.55 \mathrm{mmol}$ ) in $\mathrm{MeOH}(3 \mathrm{ml})$ at room temperature for $6 \mathrm{~h}$ followed by column chromatography afforded the product $\mathbf{4 b}$ as a yellow liquid (110 $\mathrm{mg}, 91 \%)$. $\mathbf{R}_{\mathbf{f}}$ (Ethyl acetate/Hexane : $50 / 50)=0.34 .{ }^{13} \mathrm{C} \mathrm{NMR}\left(100 \mathrm{MHz}, \delta \mathrm{ppm} / \mathrm{CDCl}_{3}\right): 159.8(\mathrm{C}), 130.7\left(\mathrm{~d}, J_{C_{-}}\right.$ $p=12.2 \mathrm{~Hz}, \mathrm{C}), 128.3(\mathrm{CH}), 128.3(\mathrm{CH}), 114.5(\mathrm{CH}), 114.5(\mathrm{CH}), 81.6(\mathrm{~d}$, $\left.J_{C-p}=158.8 \mathrm{~Hz}, \mathrm{CH}\right), 62.2(\mathrm{CH}), 55.4\left(\mathrm{~s}, \mathrm{CH}_{3}\right), 53.9\left(\mathrm{~d}, J_{\mathrm{C}-\mathrm{p}}=6.6 \mathrm{~Hz}, \mathrm{CH}_{3}\right), 53.4\left(\mathrm{~d}, J_{C-p}=6.7 \mathrm{~Hz}\right.$, $\left.\mathrm{CH}_{3}\right), 49.6\left(\mathrm{CH}_{2}\right), 21.2\left(\mathrm{CH}_{2}\right), 11.3\left(\mathrm{CH}_{3}\right) .{ }^{1} \mathrm{H} \mathrm{NMR}\left(400 \mathrm{MHz}, \delta \mathrm{ppm} / \mathrm{CDCl}_{3}\right): 7.12(\mathrm{~d}, J=8.8 \mathrm{~Hz}$, $2 \mathrm{H}), 6.86(\mathrm{~d}, J=8.8 \mathrm{~Hz}, 2 \mathrm{H}), 4.65(\mathrm{dd}, J=26.0,10.8 \mathrm{~Hz}, 1 \mathrm{H}), 4.51(\mathrm{dd}, J=17.2,11.2 \mathrm{~Hz}, 1 \mathrm{H})$, $3.82(\mathrm{~d}, J=10.4 \mathrm{~Hz}, 3 \mathrm{H}), 3.77(\mathrm{~s}, 3 \mathrm{H}), 3.76(\mathrm{~d}, J=10.4 \mathrm{~Hz}, 3 \mathrm{H}), 3.60-3.53(\mathrm{~m}, 1 \mathrm{H}), 3.28-3.21$ $(\mathrm{m}, 1 \mathrm{H}), 1.64-1.55(\mathrm{~m}, 2 \mathrm{H}), 0.87(\mathrm{t}, J=7.2 \mathrm{~Hz}, 3 \mathrm{H}) .{ }^{31} \mathrm{P}$ NMR $\left(161.9 \mathrm{MHz}, \mathrm{CDCl}_{3}\right): 21.08$. HRMS for $\mathrm{C}_{14} \mathrm{H}_{22} \mathrm{~N}_{3} \mathrm{NaO}_{4} \mathrm{P}^{+}$: calcd. [M+Na] $]^{+}: 350.1240$, found: 350.1245 . FTIR (cm $\left.{ }^{-1}\right): 3388,1617$, 1512, 1402, 1219, 1057, 835, 769.

Compound 4c: Dimethyl (5-(4-(dimethylamino)phenyl)-1-propyl-4,5-dihydro-1H-1,2,3triazol-4-yl)phosphonate

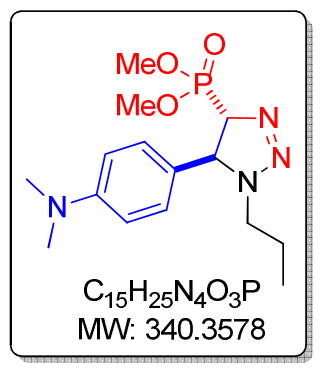

Following the general procedure, treatment of $\mathrm{N}, \mathrm{N}$ dimethyl benzaldehyde 1c (50 mg, $0.34 \mathrm{mmol}$ ) with n-propylamine $3 a(30 \mathrm{mg}$, $0.50 \mathrm{mmol}$ ) and $\mathrm{BOR}(97 \mathrm{mg}, 0.50 \mathrm{mmol}$ ) in $\mathrm{MeOH}(3 \mathrm{ml}$ ) at room 
temperature for $6 \mathrm{~h}$ followed by column chromatography afforded the product $4 \mathrm{c}$ as a yellow liquid (102 mg, 88\%). $\mathbf{R}_{\mathbf{f}}$ (Ethyl acetate/Hexane : 50/50) $=0.36 .{ }^{13} \mathrm{C} \mathbf{N M R}(100 \mathrm{MHz}, \delta$ $\mathrm{ppm} / \mathrm{CDCl}_{3}$ ): $150.7(\mathrm{C}), 128.0(\mathrm{CH}), 128.0(\mathrm{CH}), 125.9$ (d, $\left.J_{C-p}=12.8 \mathrm{~Hz}, \mathrm{C}\right), 112.6(\mathrm{CH}), 112.6$ $(\mathrm{CH}), 81.3\left(\mathrm{~d}, J_{C-P}=158.0 \mathrm{~Hz}, \mathrm{CH}\right), 62.3(\mathrm{CH}), 53.9\left(\mathrm{~d}, J_{C-P}=6.5 \mathrm{~Hz}, \mathrm{CH}_{3}\right), 53.4\left(\mathrm{~d}, J_{C-P}=6.7 \mathrm{~Hz}\right.$, $\left.\mathrm{CH}_{3}\right), 49.4\left(\mathrm{CH}_{2}\right), 40.5\left(\mathrm{~s}, \mathrm{CH}_{3}\right), 40.5\left(\mathrm{~s}, \mathrm{CH}_{3}\right), 21.3\left(\mathrm{CH}_{2}\right), 11.3\left(\mathrm{CH}_{3}\right) .{ }^{1} \mathbf{H}$ NMR $(400 \mathrm{MHz}, \delta$ $\left.\mathrm{ppm} / \mathrm{CDCl}_{3}\right): 7.05(\mathrm{~d}, J=8.8 \mathrm{~Hz}, 2 \mathrm{H}), 6.67(\mathrm{~d}, J=8.4 \mathrm{~Hz}, 2 \mathrm{H}), 4.70-4.51(\mathrm{~m}, 2 \mathrm{H}), 3.83(\mathrm{~d}, J=$ $10.4 \mathrm{~Hz}, 3 \mathrm{H}), 3.76(\mathrm{~d}, J=10.8 \mathrm{~Hz}, 3 \mathrm{H}), 3.62-3.55(\mathrm{~m}, 1 \mathrm{H}), 3.28-3.21(\mathrm{~m}, 1 \mathrm{H}), 2.94(\mathrm{~s}, 6 \mathrm{H})$, 1.66-1.56 (m, 2H), $0.88(\mathrm{t}, J=7.6 \mathrm{~Hz}, 3 \mathrm{H}) .{ }^{31} \mathrm{P}$ NMR (161.9 MHz, $\left.\mathrm{CDCl}_{3}\right): 21.47$. HRMS for $\mathrm{C}_{15} \mathrm{H}_{25} \mathrm{~N}_{4} \mathrm{NaO}_{3} \mathrm{P}^{+}$: calcd. [M+Na] $]^{+}: 363.1556$, found: 363.1545 . FTIR $\left(\mathrm{cm}^{-1}\right): 3392,1639,1403$, $1068,833,770$.

Compound 4d: (4-(4-(Dimethoxyphosphoryl)-1-propyl-4,5-dihydro-1H-1,2,3-triazol-5yl)phenyl)boronic acid

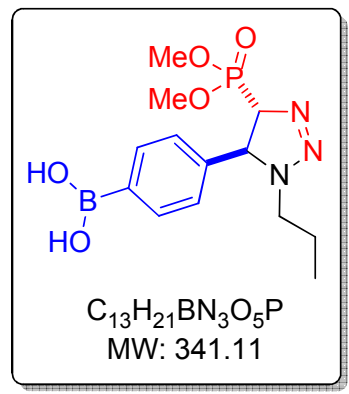

Following the general procedure, treatment of 4formylphenylboronic acid $1 \mathrm{~d}(50 \mathrm{mg}, 0.33 \mathrm{mmol}$ ) with n-propylamine $3 a$ (49 mg, $0.83 \mathrm{mmol}$ ) and BOR (96 mg, $0.50 \mathrm{mmol}$ ) in $\mathrm{MeOH}(3 \mathrm{ml})$ at room temperature for $6 \mathrm{~h}$ followed by column chromatography afforded the product $\mathbf{4} \mathbf{d}$ as a white solid (87 $\mathrm{mg}, 77 \%) . \mathbf{R}_{\mathbf{f}}$ (acetone/dichloromethane : 20/80) $=0.32 . \mathrm{Mp}=115-117{ }^{\circ} \mathrm{C} .{ }^{13} \mathrm{C}$ NMR $\left(100 \mathrm{MHz}, \delta \mathrm{ppm} / \mathrm{DMSO}-\mathrm{d}_{6}\right): 140.4\left(\mathrm{~d}, \mathrm{~J}_{C-P}=11.1 \mathrm{~Hz}, \mathrm{C}\right), 134.6$ (CH), $134.6(\mathrm{CH}), 126.4(\mathrm{CH}), 126.4(\mathrm{CH}), 80.5\left(\mathrm{~d}, J_{C-P}=157.8 \mathrm{~Hz}, \mathrm{CH}\right), 62.1(\mathrm{CH}), 53.4\left(\mathrm{~d}, J_{C-p}=\right.$ $\left.6.4 \mathrm{~Hz}, \mathrm{CH}_{3}\right), 53.1\left(\mathrm{CH}_{3}\right), 49.1\left(\mathrm{CH}_{2}\right), 20.6\left(\mathrm{CH}_{2}\right), 11.0\left(\mathrm{CH}_{3}\right) .{ }^{1} \mathrm{H}$ NMR $(400 \mathrm{MHz}, \delta \mathrm{ppm} / \mathrm{DMSO}-$ $\mathrm{d}_{6}$ ): $8.07(\mathrm{~s}, 2 \mathrm{OH}), 7.79(\mathrm{~d}, J=7.6,2 \mathrm{H}), 7.24(\mathrm{~d}, J=8.0,2 \mathrm{H}), 4.78(\mathrm{dd}, J=17.2,11.6 \mathrm{~Hz}, 1 \mathrm{H})$, $4.61(\mathrm{dd}, J=26.0,11.6 \mathrm{~Hz}, 1 \mathrm{H}), 3.72(\mathrm{~d}, J=10.8 \mathrm{~Hz}, 3 \mathrm{H}), 3.65(\mathrm{~d}, J=10.8 \mathrm{~Hz}, 3 \mathrm{H}), 3.48-3.43$ $(\mathrm{m}, 1 \mathrm{H}), 3.24-3.18(\mathrm{~m}, 1 \mathrm{H}), 1.54-1.15(\mathrm{~m}, 2 \mathrm{H}), 0.81(\mathrm{t}, J=7.6 \mathrm{~Hz}, 3 \mathrm{H}) .{ }^{31} \mathrm{P}$ NMR $(161.9 \mathrm{MHz}$, DMSO- $\left.\mathrm{d}_{6}\right):$ 21.09. HRMS for $\mathrm{C}_{13} \mathrm{H}_{21} \mathrm{BN}_{3} \mathrm{NaO}_{5} \mathrm{P}^{+}$: calcd. [M+Na] $]^{+}: 364.1204$, found: 364.1235 . FTIR $\left(\mathrm{cm}^{-1}\right)$ : 3391, 1645, 1403, 1217, 1051, 833, 771.

Compound 4e: Dimethyl (5-(3-methoxyphenyl)-1-propyl-4,5-dihydro-1H-1,2,3-triazol-4yl)phosphonate

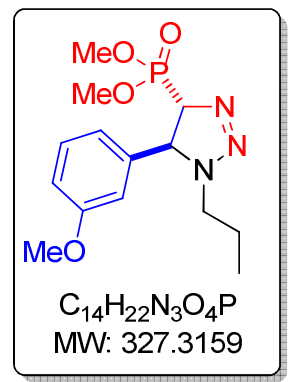

Following the general procedure, treatment of 3-methoxy benzaldehyde 1e (50 mg, $0.37 \mathrm{mmol}$ ) with n-propylamine 3 a (33 $\mathrm{mg}, 0.55 \mathrm{mmol}$ ) and BOR (106 mg, $0.55 \mathrm{mmol}$ ) in $\mathrm{MeOH}(3 \mathrm{ml}$ ) at room temperature for $6 \mathrm{~h}$ followed by column chromatography afforded the product $4 \mathbf{e}$ as a yellow liquid (89 $\mathrm{mg}, 73 \%)$. $\mathbf{R}_{\mathbf{f}}$ (Ethyl acetate/Hexane : 50/50) $=0.35 .{ }^{13} \mathbf{C} \mathbf{~ N M R}$ $\left(100 \mathrm{MHz}, \delta \mathrm{ppm} / \mathrm{CDCl}_{3}\right): 160.3$ (C), $140.6\left(\mathrm{~d}, J_{C-P}=11.9 \mathrm{~Hz}, \mathrm{C}\right), 130.3$ $(\mathrm{CH}), 119.3(\mathrm{CH}), 114.2(\mathrm{CH}), 112.4(\mathrm{CH}), 81.8\left(\mathrm{~d}, J_{\mathrm{C}-\mathrm{p}}=159.0 \mathrm{~Hz}, \mathrm{CH}\right), 62.5$

$(\mathrm{CH}), 55.4\left(\mathrm{~s}, \mathrm{CH}_{3}\right), 54.0\left(\mathrm{~d}, J_{C-P}=6.6 \mathrm{~Hz}, \mathrm{CH}_{3}\right), 53.4\left(\mathrm{~d}, J_{C-P}=6.8 \mathrm{~Hz}, \mathrm{CH}_{3}\right), 49.8\left(\mathrm{CH}_{2}\right), 21.2$ $\left(\mathrm{CH}_{2}\right), 11.3\left(\mathrm{CH}_{3}\right) .{ }^{1} \mathrm{H}$ NMR $\left(400 \mathrm{MHz}, \delta \mathrm{ppm} / \mathrm{CDCl}_{3}\right): 7.25(\mathrm{dd}, J=8.0,5.6 \mathrm{~Hz}, 1 \mathrm{H}), 6.85-6.69$ $(\mathrm{m}, 2 \mathrm{H}), 6.73(\mathrm{~d}, J=2.0 \mathrm{~Hz}, 1 \mathrm{H}), 4.71-4.52(\mathrm{~m}, 2 \mathrm{H}), 3.84(\mathrm{~d}, J=10.4 \mathrm{~Hz}, 3 \mathrm{H}), 3.78(\mathrm{~d}, J=10.4$ 
$\mathrm{Hz}, 3 \mathrm{H}), 3.77(\mathrm{~s}, 3 \mathrm{H}), 3.65-3.58(\mathrm{~m}, 1 \mathrm{H}), 3.33-3.26(\mathrm{~m}, 1 \mathrm{H}), 1.66-1.57(\mathrm{~m}, 2 \mathrm{H}), 0.89(\mathrm{t}, J=7.2$ $\mathrm{Hz}, 3 \mathrm{H}) .{ }^{31} \mathrm{P}$ NMR (161.9 MHz, $\left.\mathrm{CDCl}_{3}\right)$ : 20.88. HRMS for $\mathrm{C}_{14} \mathrm{H}_{22} \mathrm{~N}_{3} \mathrm{NaO}_{4} \mathrm{P}^{+}$: calcd. $[\mathrm{M}+\mathrm{Na}]^{+}$: 350.1240, found: 350.1234. FTIR ( $\left.\mathrm{cm}^{-1}\right)$ : 3854, 3746, 3392, 3017, 1626, 1459, 1403, 1218, $1051,831,771,666,559$.

Compound 4f: Dimethyl (5-(3-nitrophenyl)-1-propyl-4,5-dihydro-1H-1,2,3-triazol-4yl)phosphonate

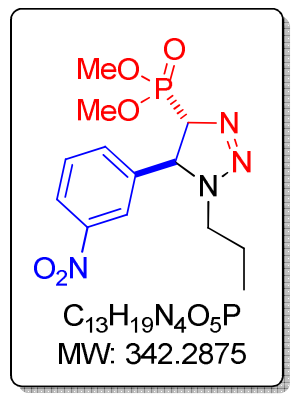

Following the general procedure, treatment of 3-nitro benzaldehyde $\mathbf{1 f}$ ( $50 \mathrm{mg}, 0.33 \mathrm{mmol}$ ) with n-propylamine $3 a(29 \mathrm{mg}, 0.50 \mathrm{mmol}$ ) and BOR (95 $\mathrm{mg}, 0.50 \mathrm{mmol}$ ) in $\mathrm{MeOH}(3 \mathrm{ml}$ ) at room temperature for $6 \mathrm{~h}$ followed by column chromatography afforded the product $\mathbf{4 f}$ as a yellow liquid (34 mg, 30\%). $\mathbf{R}_{\mathbf{f}}$ (Ethyl acetate/Hexane : 50/50) $=0.45 .{ }^{13} \mathbf{C ~ N M R}$ $\left(100 \mathrm{MHz}, \delta \mathrm{ppm} / \mathrm{CDCl}_{3}\right): 148.9(\mathrm{C}), 141.6\left(\mathrm{~d}, J_{\mathrm{C}-\mathrm{P}}=10.8 \mathrm{~Hz}, \mathrm{C}\right), 133.1(\mathrm{CH})$, $130.5(\mathrm{CH}), 123.7(\mathrm{CH}), 122.1(\mathrm{CH}), 82.6\left(\mathrm{~d}, J_{\mathrm{C}-\mathrm{p}}=160.6 \mathrm{~Hz}, \mathrm{CH}\right), 62.2(\mathrm{CH})$,

$54.3\left(\mathrm{CH}_{3}\right), 53.6\left(\mathrm{CH}_{3}\right), 50.5\left(\mathrm{CH}_{2}\right), 21.2\left(\mathrm{CH}_{2}\right), 11.3\left(\mathrm{CH}_{3}\right) .{ }^{1} \mathrm{H} \mathrm{NMR}\left(400 \mathrm{MHz}, \delta \mathrm{ppm} / \mathrm{CDCl}_{3}\right)$ : $8.20(\mathrm{~d}, J=7.2 \mathrm{~Hz}, 1 \mathrm{H}), 8.14(\mathrm{~s}, 1 \mathrm{H}), 7.63-7.56(\mathrm{~m}, 2 \mathrm{H}), 4.78(\mathrm{dd}, J=26.0,11.6 \mathrm{~Hz}, 1 \mathrm{H}), 4.51$ (dd, $J=18.0,11.6 \mathrm{~Hz}, 1 \mathrm{H}), 3.89$ (d, $J=10.8 \mathrm{~Hz}, 3 \mathrm{H}), 3.83(\mathrm{~d}, J=10.8 \mathrm{~Hz}, 3 \mathrm{H}), 3.90-3.82(\mathrm{~m}$, $1 \mathrm{H}), 3.36-3.29(\mathrm{~m}, 1 \mathrm{H}), 1.68-1.62(\mathrm{~m}, 2 \mathrm{H}), 0.95(\mathrm{t}, J=7.2 \mathrm{~Hz}, 3 \mathrm{H}) .{ }^{31} \mathbf{P}$ NMR $(161.9 \mathrm{MHz}$, $\mathrm{CDCl}_{3}$ ): 19.96. HRMS for $\mathrm{C}_{13} \mathrm{H}_{19} \mathrm{~N}_{4} \mathrm{NaO}_{5} \mathrm{P}^{+}$: calcd. [M+Na] ${ }^{+}: 365.0985$, found: 365.0977. FTIR $\left(\mathrm{cm}^{-1}\right):$ 3391, 3020, 2400, 1626, 1535, 1404, 1353, 1261, 1216, 1055, 929, 770, 669.

Compound 4g: Dimethyl (1-propyl-5-(3,4,5-trimethoxyphenyl)-4,5-dihydro-1H-1,2,3triazol-4-yl)phosphonate

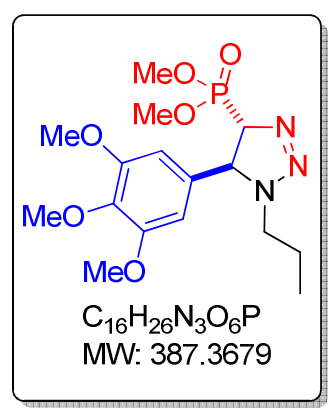

Following the general procedure, treatment of $3,4,5$ trimethoxy benzaldehyde $1 \mathrm{~g}$ (50 $\mathrm{mg}, 0.25 \mathrm{mmol}$ ) with n-propylamine $3 \mathrm{a}$ ( $23 \mathrm{mg}$, $0.38 \mathrm{mmol}$ ) and $\mathrm{BOR}(73 \mathrm{mg}, 0.38 \mathrm{mmol})$ in $\mathrm{MeOH}(3 \mathrm{ml})$ at room temperature for $6 \mathrm{~h}$ followed by column chromatography afforded the product $\mathbf{4 g}$ as a yellow liquid $(89 \mathrm{mg}, \mathbf{9 2 \%}) . \mathbf{R}_{\mathbf{f}}$ (Ethyl acetate/Hexane : $50 / 50)=0.20 .{ }^{13} \mathrm{C}$ NMR $\left(100 \mathrm{MHz}, \delta \mathrm{ppm} / \mathrm{CDCl}_{3}\right): 153.8$ (C), 153.8 (C), $138.0(\mathrm{C}), 134.6\left(\mathrm{~d}, J_{C-P}=12.1 \mathrm{~Hz}, \mathrm{C}\right), 103.6(\mathrm{CH}), 103.6(\mathrm{CH}), 81.7\left(\mathrm{~d}, J_{C-P}\right.$ $=158.6 \mathrm{~Hz}, \mathrm{CH}), 62.8(\mathrm{CH}), 60.9\left(\mathrm{~s}, \mathrm{CH}_{3}\right), 56.3\left(\mathrm{~s}, \mathrm{CH}_{3}\right), 56.3\left(\mathrm{~s}, \mathrm{CH}_{3}\right)$, $54.1\left(\mathrm{~d}, J_{C-P}=6.7 \mathrm{~Hz}, \mathrm{CH}_{3}\right), 53.5\left(\mathrm{~d}, \mathrm{~J}_{\mathrm{C}-\mathrm{P}}=6.7 \mathrm{~Hz}, \mathrm{CH}_{3}\right), 49.9\left(\mathrm{CH}_{2}\right), 21.2\left(\mathrm{CH}_{2}\right), 11.3\left(\mathrm{CH}_{3}\right) .{ }^{1} \mathbf{H}$ NMR $\left(400 \mathrm{MHz}, \delta \mathrm{ppm} / \mathrm{CDCl}_{3}\right): 6.40(\mathrm{~s}, 2 \mathrm{H}), 4.67-4.51(\mathrm{~m}, 2 \mathrm{H}), 3.85(\mathrm{~d}, J=10.8 \mathrm{~Hz}, 3 \mathrm{H}), 3.81$ (s, 9H), $3.80(\mathrm{~d}, J=10.8 \mathrm{~Hz}, 3 \mathrm{H}), 3.66-3.58(\mathrm{~m}, 1 \mathrm{H}), 3.35-3.28(\mathrm{~m}, 1 \mathrm{H}), 1.68-1.59(\mathrm{~m}, 2 \mathrm{H}), 0.91$ $(\mathrm{t}, J=7.6 \mathrm{~Hz}, 3 \mathrm{H}) .{ }^{31} \mathrm{P}$ NMR (161.9 MHz, $\mathrm{CDCl}_{3}$ ): 20.88. HRMS for $\mathrm{C}_{16} \mathrm{H}_{26} \mathrm{~N}_{3} \mathrm{NaO}_{6} \mathrm{P}^{+}$: calcd. $[\mathrm{M}+\mathrm{Na}]^{+}:$410.1451, found: 410.1454. FTIR $\left(\mathrm{cm}^{-1}\right): 3400,3019,1626,1508,1464,1404,1331$, 1217, 1129, 1054, 832, 771, 669. 
Compound 4h: Dimethyl (5-(2-hydroxyphenyl)-1-propyl-4,5-dihydro-1H-1,2,3-triazol-4yl)phosphonate

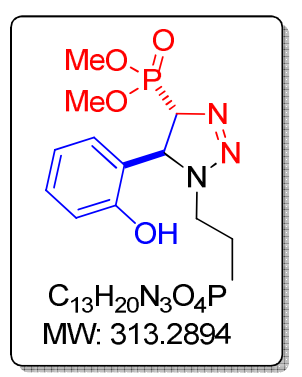

Following the general procedure, treatment of 2-hydroxy benzaldehyde 1h (50 mg, $0.41 \mathrm{mmol}$ ) with n-propylamine $3 a(36 \mathrm{mg}, 0.61 \mathrm{mmol}$ ) and BOR (118 mg, $0.61 \mathrm{mmol}$ ) in $\mathrm{MeOH}(3 \mathrm{ml})$ at room temperature for $6 \mathrm{~h}$ followed by column chromatography afforded the product $4 \mathrm{~h}$ as a yellow solid (85 mg, 66\%). $\mathbf{R}_{\mathbf{f}}$ (Ethyl acetate/Hexane : 50/50) = 0.25. $\mathbf{M p}=95-97$ ${ }^{\circ} \mathrm{C} .{ }^{13} \mathrm{C}$ NMR $\left(100 \mathrm{MHz}, \delta \mathrm{ppm} / \mathrm{CDCl}_{3}\right): 155.3(\mathrm{C}), 129.7(\mathrm{CH}), 127.3(\mathrm{CH})$, $124.7\left(\mathrm{~d}, J_{C-P}=10.5 \mathrm{~Hz}, \mathrm{C}\right), 120.9(\mathrm{CH}), 118.1(\mathrm{CH}), 80.0\left(\mathrm{~d}, J_{C-P}=158.5 \mathrm{~Hz}\right.$, $\mathrm{CH}), 57.3(\mathrm{CH}), 54.3\left(\mathrm{~d}, J_{C-P}=7.1 \mathrm{~Hz}, \mathrm{CH}_{3}\right), 54.0\left(\mathrm{~d}, J_{C-P}=6.6 \mathrm{~Hz}, \mathrm{CH}_{3}\right), 50.0\left(\mathrm{CH}_{2}\right), 21.0\left(\mathrm{CH}_{2}\right)$, $11.3\left(\mathrm{CH}_{3}\right) .{ }^{1} \mathrm{H}$ NMR $\left(400 \mathrm{MHz}, \delta \mathrm{ppm} / \mathrm{CDCl}_{3}\right): 8.36(\mathrm{~s}, \mathrm{OH}), 7.19-7.15(\mathrm{~m}, 1 \mathrm{H}), 7.07(\mathrm{~d}, J=7.6$ $\mathrm{Hz}, 1 \mathrm{H}), 6.97(\mathrm{~d}, J=8.0 \mathrm{~Hz}, 1 \mathrm{H}), 6.89(\mathrm{t}, J=7.2,1 \mathrm{H}), 5.08(\mathrm{dd}, J=27.6,11.6 \mathrm{~Hz}, 1 \mathrm{H}), 4.60$ (dd, $J=16.0,11.6 \mathrm{~Hz}, 1 \mathrm{H}), 3.89(\mathrm{~d}, J=8.8 \mathrm{~Hz}, 3 \mathrm{H}), 3.86(\mathrm{~d}, J=9.2 \mathrm{~Hz}, 3 \mathrm{H}), 3.64-3.61(\mathrm{~m}, 1 \mathrm{H}), 3.32-$ $3.25(\mathrm{~m}, 1 \mathrm{H}), 1.70-1.60(\mathrm{~m}, 2 \mathrm{H}), 0.90(\mathrm{t}, J=7.2 \mathrm{~Hz}, 3 \mathrm{H}) .{ }^{31} \mathrm{P}$ NMR $\left(161.9 \mathrm{MHz}, \mathrm{CDCl}_{3}\right): 22.66$. HRMS for $\mathrm{C}_{13} \mathrm{H}_{20} \mathrm{~N}_{3} \mathrm{NaO}_{4} \mathrm{P}^{+}$: calcd. [M+Na] $]^{+}: 336.1084$, found: 336.1093. FTIR (cm $\left.{ }^{-1}\right): 3391$, 3020, 1638, 1403, 1217, 1053, 832, 771, 669.

Compound 4i: Dimethyl (5-(3-ethoxy-4-hydroxyphenyl)-1-propyl-4,5-dihydro-1H-1,2,3triazol-4-yl)phosphonate

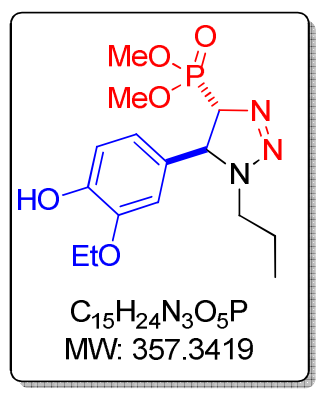

Following the general procedure, treatment of 3-ethoxy, 4hydroxybenzaldehyde $1 \mathbf{i}(50 \mathrm{mg}, 0.30 \mathrm{mmol}$ ) with n-propylamine $3 \mathbf{a}$ ( 27 $\mathrm{mg}, 0.45 \mathrm{mmol}$ ) and $\mathrm{BOR}(87 \mathrm{mg}, 0.45 \mathrm{mmol})$ in $\mathrm{MeOH}(3 \mathrm{ml})$ at room temperature for $6 \mathrm{~h}$ followed by column chromatography afforded the product $\mathbf{4} \mathbf{i}$ as a yellow solid (102 $\mathrm{mg}, 95 \%)$. $\mathbf{R}_{\mathbf{f}}$ (Ethyl acetate/Hexane : $50 / 50)=0.19 . \mathrm{Mp}=90-92{ }^{\circ} \mathrm{C} .{ }^{13} \mathrm{C}$ NMR $\left(100 \mathrm{MHz}, \delta \mathrm{ppm} / \mathrm{CDCl}_{3}\right): 146.6$ (C), $146.3(\mathrm{C}), 130.5\left(\mathrm{~d}, J_{\mathrm{C}-\mathrm{p}}=12.2 \mathrm{~Hz}, \mathrm{C}\right), 120.2(\mathrm{CH}), 114.8(\mathrm{CH}), 109.9$ $(\mathrm{CH}), 81.6\left(\mathrm{~d}, J_{C-P}=158.7 \mathrm{~Hz}, \mathrm{CH}\right), 64.7\left(\mathrm{CH}_{2}\right), 62.6(\mathrm{CH}), 54.0\left(\mathrm{~d}, J_{C-P}=6.8 \mathrm{~Hz}, \mathrm{CH}_{3}\right), 53.4\left(\mathrm{~d}, J_{C-}\right.$ $\left.p=6.8 \mathrm{~Hz}, \mathrm{CH}_{3}\right), 49.7\left(\mathrm{CH}_{2}\right), 21.2\left(\mathrm{CH}_{2}\right), 14.8\left(\mathrm{CH}_{2}\right), 11.3\left(\mathrm{CH}_{3}\right) .{ }^{1} \mathbf{H}$ NMR $(400 \mathrm{MHz}, \delta$ $\left.\mathrm{ppm} / \mathrm{CDCl}_{3}\right): 6.86(\mathrm{~d}, J=8.0 \mathrm{~Hz}, 1 \mathrm{H}), 6.70(\mathrm{dd}, J=8.4,2.0 \mathrm{~Hz}, 1 \mathrm{H}), 6.64(\mathrm{~s}, 1 \mathrm{H}), 5.94(\mathrm{~s}, \mathrm{OH})$, 4.67-4.49 (m, 2H), 4.05 (q, $J=6.8 \mathrm{~Hz}, 2 \mathrm{H}), 3.83$ (d, $J=10.4 \mathrm{~Hz}, 3 \mathrm{H}), 3.77$ (d, $J=10.8 \mathrm{~Hz}, 3 \mathrm{H}$ ), 3.59-3.56 (m, $1 \mathrm{H}), 3.31-3.24(\mathrm{~m}, 1 \mathrm{H}), 1.65-1.56(\mathrm{~m}, 2 \mathrm{H}), 1.41(\mathrm{t}, J=6.8 \mathrm{~Hz}, 3 \mathrm{H}), 0.89(\mathrm{t}, J=$ $7.2 \mathrm{~Hz}, 3 \mathrm{H}) .{ }^{31} \mathrm{P}$ NMR $\left(161.9 \mathrm{MHz}, \mathrm{CDCl}_{3}\right)$ : 21.08. HRMS for $\mathrm{C}_{15} \mathrm{H}_{24} \mathrm{~N}_{3} \mathrm{NaO}_{5} \mathrm{P}^{+}$: calcd. $[\mathrm{M}+\mathrm{Na}]^{+}$: 380.1346, found: 380.1344. FTIR ( $\left.\mathrm{cm}^{-1}\right)$ : 3172, 1638, 1403, 1068, 833, 769.

Compound 4j: Dimethyl (5-(3,4-dihydroxyphenyl)-1-propyl-4,5-dihydro-1H-1,2,3-triazol-4yl)phosphonate

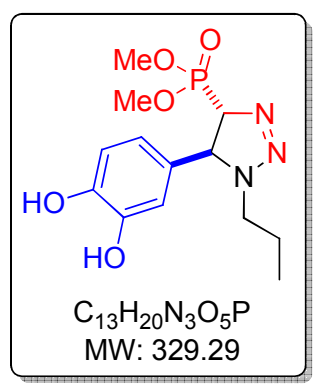

Following the general procedure, treatment of 3, 4-dihydroxy benzaldehyde $1 \mathbf{j}$ (50 mg, $0.36 \mathrm{mmol}$ ) with $\mathrm{n}$-propylamine $3 \mathbf{3}$ (32 $\mathrm{mg}$, $0.54 \mathrm{mmol}$ ) and BOR (104 $\mathrm{mg}, 0.54 \mathrm{mmol})$ in $\mathrm{MeOH}(3 \mathrm{ml})$ at room temperature for $6 \mathrm{~h}$ followed by column chromatography afforded the 
product $\mathbf{4} \mathbf{j}$ as a yellow solid (70 $\mathrm{mg}, 59 \%) . \mathbf{R}_{\mathbf{f}}$ (Ethyl acetate/Hexane : $\left.50 / 50\right)=0.15 . \mathbf{M p}=$ 110-112 ${ }^{\circ} \mathrm{C} .{ }^{13} \mathrm{C}$ NMR $\left(100 \mathrm{MHz}, \delta \mathrm{ppm} / \mathrm{CDCl}_{3}\right): 145.6(\mathrm{C}), 145.4(\mathrm{C}), 130.0$ (d, J J $\mathrm{J}_{\mathrm{p}}=13.6 \mathrm{~Hz}$, C), $119.2(\mathrm{CH}), 115.4(\mathrm{CH}), 113.2(\mathrm{CH}), 80.2\left(\mathrm{~d}, J_{C-P}=158.4 \mathrm{~Hz}, \mathrm{CH}\right), 62.7(\mathrm{CH}), 54.3\left(\mathrm{~d}, J_{C-p}=\right.$ $\left.6.8 \mathrm{~Hz}, \mathrm{CH}_{3}\right), 54.0\left(\mathrm{~d}, \mathrm{~J}_{\mathrm{C}-\mathrm{P}}=6.9 \mathrm{~Hz}, \mathrm{CH}_{3}\right), 49.4\left(\mathrm{CH}_{2}\right), 21.3\left(\mathrm{CH}_{2}\right), 11.3\left(\mathrm{CH}_{3}\right) .{ }^{1} \mathrm{H} \mathbf{N M R}(400 \mathrm{MHz}$, $\left.\delta \mathrm{ppm} / \mathrm{CDCl}_{3}\right): 8.41(\mathrm{~s}, \mathrm{OH}), 6.80(\mathrm{~d}, J=8.0 \mathrm{~Hz}, 1 \mathrm{H}), 6.68(\mathrm{~d}, J=0.8 \mathrm{~Hz}, 1 \mathrm{H}), 6.60-6.58(\mathrm{~m}, 1 \mathrm{H})$, 4.69-4.59 (m, 2H), $3.85(\mathrm{~d}, J=10.8 \mathrm{~Hz}, 3 \mathrm{H}), 3.81(\mathrm{~d}, J=10.8 \mathrm{~Hz}, 3 \mathrm{H}), 3.65-3.58(\mathrm{~m}, 1 \mathrm{H}), 3.32-$ $3.25(\mathrm{~m}, 1 \mathrm{H}), 1.64-1.58(\mathrm{~m}, 2 \mathrm{H}), 0.89(\mathrm{t}, J=7.2 \mathrm{~Hz}, 3 \mathrm{H}) .{ }^{31} \mathrm{P}$ NMR $\left(161.9 \mathrm{MHz}, \mathrm{CDCl}_{3}\right): 20.94$. HRMS for $\mathrm{C}_{13} \mathrm{H}_{20} \mathrm{~N}_{3} \mathrm{NaO}_{5} \mathrm{P}^{+}$: calcd. [M+Na] $]^{+}: 352.1033$, found: 352.1030 . FTIR $\left(\mathrm{cm}^{-1}\right)$ : 3392, 3020, 1638, 1403, 1265, 1217, 1048, 770, 669.

Compound 4k: Dimethyl (5-ferrocen-2-yl)-1-propyl-4,5-dihydro-1H-1,2,3-triazol-4yl)phosphonate

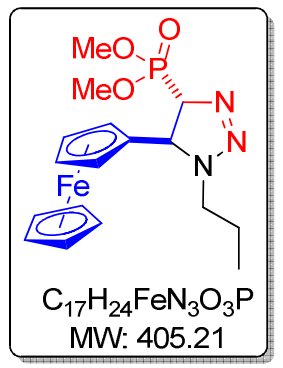

Following the general procedure, treatment of ferrocine carboxaldehyde 1k (50 $\mathrm{mg}, 0.23 \mathrm{mmol}$ ) with n-propylamine $3 a(21 \mathrm{mg}, 0.35 \mathrm{mmol}$ ) and BOR (67 mg, $0.35 \mathrm{mmol}$ ) in $\mathrm{MeOH}(3 \mathrm{ml}$ ) at room temperature for $6 \mathrm{~h}$ followed by column chromatography afforded the product $\mathbf{4 k}$ as a yellow liquid (72 mg, 77\%). $\mathbf{R}_{\mathbf{f}}$ (Ethyl acetate/Hexane : 50/50) $=0.40 .{ }^{13} \mathbf{C} \mathbf{~ N M R}$ $\left(100 \mathrm{MHz}, \delta \mathrm{ppm} / \mathrm{CDCl}_{3}\right): 86.5\left(\mathrm{~d}, J_{C-P}=21.5 \mathrm{~Hz}, \mathrm{C}\right), 79.9$ (d, $J_{C-P}=151.3 \mathrm{~Hz}$, $\mathrm{CH}), 69.4(\mathrm{CH}), 68.9(\mathrm{CH}), 68.9(\mathrm{CH}), 68.9(\mathrm{CH}), 68.9(\mathrm{CH}), 68.9(\mathrm{CH}), 68.9$ $(\mathrm{CH}), 68.2(\mathrm{CH}), 65.2(\mathrm{CH}), 57.9(\mathrm{CH}), 54.0\left(\mathrm{~d}, J_{C-P}=6.7 \mathrm{~Hz}, \mathrm{CH}_{3}\right), 53.6\left(\mathrm{~d}, J_{C-P}=6.8 \mathrm{~Hz}, \mathrm{CH}_{3}\right)$, $48.5\left(\mathrm{CH}_{2}\right), 21.6\left(\mathrm{CH}_{2}\right), 11.3\left(\mathrm{CH}_{3}\right) .{ }^{1} \mathrm{H}$ NMR $\left(400 \mathrm{MHz}, \delta \mathrm{ppm} / \mathrm{CDCl}_{3}\right): 5.17$ (dd, $J=17.2,4.8$ $\mathrm{Hz}, 1 \mathrm{H}), 4.64(\mathrm{dd}, J=26.4,5.2 \mathrm{~Hz}, 1 \mathrm{H}), 4.26(\mathrm{~s}, 5 \mathrm{H}), 4.18(\mathrm{~s}, 1 \mathrm{H}), 4.14(\mathrm{~s}, 2 \mathrm{H}), 3.91(\mathrm{~d}, J=10.8$ $\mathrm{Hz}, 3 \mathrm{H}), 3.89(\mathrm{~s}, 1 \mathrm{H}), 3.82(\mathrm{~d}, J=10.4 \mathrm{~Hz}, 3 \mathrm{H}), 3.57-3.52(\mathrm{~m}, 1 \mathrm{H}), 3.15-3.08(\mathrm{~m}, 1 \mathrm{H}), 1.65-1.58$ $(\mathrm{m}, 2 \mathrm{H}), 0.90(\mathrm{t}, J=7.2 \mathrm{~Hz}, 3 \mathrm{H}) .{ }^{31} \mathrm{P}$ NMR $\left(161.9 \mathrm{MHz}, \mathrm{CDCl}_{3}\right)$ : 21.49. HRMS for $\mathrm{C}_{17} \mathrm{H}_{24} \mathrm{FeN}_{3} \mathrm{NaO}_{3} \mathrm{P}^{+}$: calcd. [M+Na] $]^{+}: 428.0797$, found: 428.0794. FTIR $\left(\mathrm{cm}^{-1}\right): 3392,3018$, 2927, 1654, 1403, 1218, 1157, 1051, 832, 772, 669.

\section{Compound 4I: Dimethyl (1-propyl-5-(thiophen-3-yl)-4,5-dihydro-1H-1,2,3-triazol-4- yl)phosphonate}

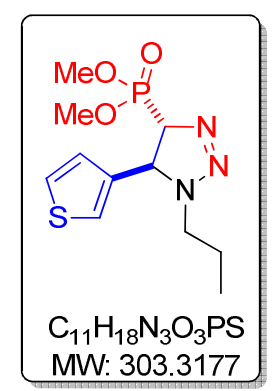

Following the general procedure, treatment of thiophene-3carboxaldehyde 1l (50 mg, $0.45 \mathrm{mmol})$ with n-propylamine 3 a $(40 \mathrm{mg}, 0.67$ $\mathrm{mmol}$ ) and $\mathrm{BOR}(129 \mathrm{mg}, 0.67 \mathrm{mmol})$ in $\mathrm{MeOH}(3 \mathrm{ml})$ at room temperature for $6 \mathrm{~h}$ followed by column chromatography afforded the product $4 \mathrm{l}$ as a greenish liquid (91 mg, 67\%). $\mathbf{R}_{\mathbf{f}}$ (Ethyl acetate/Hexane : 50/50) $=0.43 .{ }^{13} \mathbf{C}$ NMR $\left(100 \mathrm{MHz}, \delta \mathrm{ppm} / \mathrm{CDCl}_{3}\right): 139.5\left(\mathrm{~d}, J_{C-p}=12.8 \mathrm{~Hz}, \mathrm{C}\right), 127.6(\mathrm{CH}), 125.6$ $(\mathrm{CH}), 123.2(\mathrm{CH}), 80.6\left(\mathrm{~d}, J_{C-P}=158.7 \mathrm{~Hz}, \mathrm{CH}\right), 58.3(\mathrm{CH}), 54.0\left(\mathrm{~d}, J_{C-P}=6.5\right.$ $\left.\mathrm{Hz}, \mathrm{CH}_{3}\right), 53.4\left(\mathrm{~d}, \mathrm{~J}_{\mathrm{C}-\mathrm{P}}=6.7 \mathrm{~Hz}, \mathrm{CH}_{3}\right), 49.8\left(\mathrm{CH}_{2}\right), 21.3\left(\mathrm{CH}_{2}\right), 11.3\left(\mathrm{CH}_{3}\right) .{ }^{1} \mathrm{H}$ NMR $(400 \mathrm{MHz}, \delta$ ppm/ $\left.\mathrm{CDCl}_{3}\right): 7.33-7.31(\mathrm{~m}, 1 \mathrm{H}), 7.20(\mathrm{dd}, J=2.8,0.8 \mathrm{~Hz}, 1 \mathrm{H}), 6.91(\mathrm{dd}, J=3.6,1.2 \mathrm{~Hz}, 1 \mathrm{H})$, $4.83(\mathrm{dd}, J=25.6,10.4 \mathrm{~Hz}, 1 \mathrm{H}$ ), 4.56 (dd, $J=17.6,10.8 \mathrm{~Hz}, 1 \mathrm{H}$ ), 3.83 (d, $J=10.8 \mathrm{~Hz}, 3 \mathrm{H}$ ), 3.78 $(\mathrm{d}, J=10.8 \mathrm{~Hz}, 3 \mathrm{H}), 3.62-3.54(\mathrm{~m}, 1 \mathrm{H}), 3.31-3.24(\mathrm{~m}, 1 \mathrm{H}), 1.65-1.59(\mathrm{~m}, 2 \mathrm{H}), 0.89(\mathrm{t}, J=7.2$ 
$\mathrm{Hz}$, 3H). ${ }^{31} \mathrm{P}$ NMR (161.9 MHz, $\left.\mathrm{CDCl}_{3}\right)$ : 20.87. HRMS for $\mathrm{C}_{11} \mathrm{H}_{18} \mathrm{~N}_{3} \mathrm{NaO}_{3} \mathrm{PS}^{+}$: calcd. $[\mathrm{M}+\mathrm{Na}]^{+}: 326.0699$, found: 326.0691. FTIR $\left(\mathrm{cm}^{-1}\right): 3392,3019,1647,1403,1218,1052,832$, $771,668,560$.

Compound 4m: Dimethyl (1-propyl-5-(thiophen-2-yl)-4,5-dihydro-1H-1,2,3-triazol-4yl)phosphonate

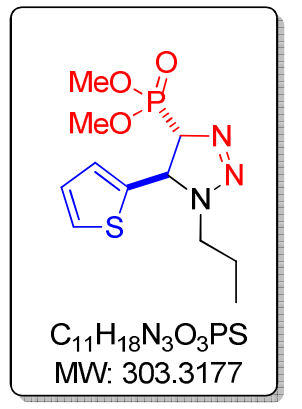

Following the general procedure, treatment of thiophene-2carboxaldehyde $1 \mathrm{~m}$ (50 mg, $0.45 \mathrm{mmol}$ ) with n-propylamine $3 a(40 \mathrm{mg}$, $0.67 \mathrm{mmol}$ ) and $\mathrm{BOR}(129 \mathrm{mg}, 0.67 \mathrm{mmol})$ in $\mathrm{MeOH}(3 \mathrm{ml})$ at room temperature for $6 \mathrm{~h}$ followed by column chromatography afforded the product $\mathbf{4 m}$ as a greenish liquid ( $82 \mathrm{mg}, 60 \%$ ). $\mathbf{R}_{\mathbf{f}}$ (Ethyl acetate/Hexane : $50 / 50)=0.42 .{ }^{13} \mathrm{C}$ NMR $\left(100 \mathrm{MHz}, \delta \mathrm{ppm} / \mathrm{CDCl}_{3}\right): 141.8\left(\mathrm{~d}, J_{C-P}=13.3 \mathrm{~Hz}\right.$, C), $127.3(\mathrm{CH}), 126.7(\mathrm{CH}), 126.2(\mathrm{CH}), 81.8\left(\mathrm{~d}, J_{C-P}=159.8 \mathrm{~Hz}, \mathrm{CH}\right), 58.6$ $(\mathrm{CH}), 54.1\left(\mathrm{~d}, J_{C-P}=6.5 \mathrm{~Hz}, \mathrm{CH}_{3}\right), 53.5\left(\mathrm{~d}, J_{C-P}=6.5 \mathrm{~Hz}, \mathrm{CH}_{3}\right), 49.9\left(\mathrm{CH}_{2}\right), 21.2\left(\mathrm{CH}_{2}\right), 11.3\left(\mathrm{CH}_{3}\right)$. ${ }^{1} \mathrm{H}$ NMR $\left(400 \mathrm{MHz}, \delta \mathrm{ppm} / \mathrm{CDCl}_{3}\right): 7.28(\mathrm{dd}, J=5.2,0.8 \mathrm{~Hz}, 1 \mathrm{H}), 7.01(\mathrm{dd}, J=3.6,1.2 \mathrm{~Hz}, 1 \mathrm{H})$, $6.96(\mathrm{dd}, J=5.2,3.6 \mathrm{~Hz}, 1 \mathrm{H}), 4.98(\mathrm{dd}, J=25.2,11.2 \mathrm{~Hz}, 1 \mathrm{H}), 4.63(\mathrm{dd}, J=17.6,11.2 \mathrm{~Hz}, 1 \mathrm{H})$, $3.85(\mathrm{~d}, J=10.8 \mathrm{~Hz}, 3 \mathrm{H}), 3.78(\mathrm{~d}, J=10.8 \mathrm{~Hz}, 3 \mathrm{H}), 3.63-3.55(\mathrm{~m}, 1 \mathrm{H}), 3.36-3.30(\mathrm{~m}, 1 \mathrm{H}), 1.69-$ $1.63(\mathrm{~m}, 2 \mathrm{H}), 0.91(\mathrm{t}, J=7.2 \mathrm{~Hz}, 3 \mathrm{H}) .{ }^{31} \mathrm{P}$ NMR (161.9 MHz, CDCl $)$ : 20.96. HRMS for $\mathrm{C}_{11} \mathrm{H}_{18} \mathrm{~N}_{3} \mathrm{NaO}_{3} \mathrm{PS}^{+}$: calcd. [M+Na] $]^{+}: 326.0699$, found: 326.0692. FTIR $\left(\mathrm{cm}^{-1}\right): 3391,3020,2400$, $1650,1403,1216,1052,929,832,771,669$.

Compound 4n: Dimethyl (5-(furan-2-yl)-1-propyl-4,5-dihydro-1H-1,2,3-triazol-4yl)phosphonate

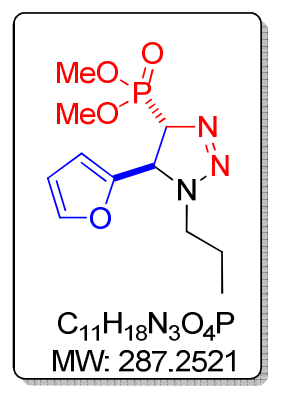

Following the general procedure, treatment of furan-2-carboxaldehyde 1n (50 $\mathrm{mg}, 0.52 \mathrm{mmol}$ ) with n-propylamine $3 a(46 \mathrm{mg}, 0.78 \mathrm{mmol}$ ) and BOR (150 $\mathrm{mg}, 0.78 \mathrm{mmol}$ ) in $\mathrm{MeOH}(3 \mathrm{ml}$ ) at room temperature for $6 \mathrm{~h}$ followed by column chromatography afforded the product $\mathbf{4 n}$ as a orange liquid (110 mg, 74\%). $\mathbf{R}_{\mathbf{f}}$ (Ethyl acetate/Hexane : 50/50) =0.39. ${ }^{13} \mathbf{C}$ NMR $\left(100 \mathrm{MHz}, \delta \mathrm{ppm} / \mathrm{CDCl}_{3}\right): 149.8\left(\mathrm{~d}, J_{C-p}=14.2 \mathrm{~Hz}, \mathrm{C}\right), 143.4(\mathrm{CH}), 110.7$ $(\mathrm{CH}), 109.4(\mathrm{CH}), 77.6\left(\mathrm{~d}, J_{C-P}=159.6 \mathrm{~Hz}, \mathrm{CH}\right), 56.1(\mathrm{CH}), 54.0\left(\mathrm{~d}, J_{C-P}=6.6\right.$ $\left.\mathrm{Hz}, \mathrm{CH}_{3}\right), 53.5\left(\mathrm{~d}, \mathrm{~J}_{\mathrm{C}-\mathrm{p}}=6.7 \mathrm{~Hz}, \mathrm{CH}_{3}\right), 49.6\left(\mathrm{CH}_{2}\right), 21.3\left(\mathrm{CH}_{2}\right), 11.2\left(\mathrm{CH}_{3}\right) .{ }^{1} \mathbf{H} \mathbf{N M R}(400 \mathrm{MHz}, \delta$ $\left.\mathrm{ppm} / \mathrm{CDCl}_{3}\right)$ : 7.36-7.35 (m, $\left.1 \mathrm{H}\right), 6.30(\mathrm{~s}, 2 \mathrm{H}), 4.85-4.72(\mathrm{~m}, 2 \mathrm{H}), 3.81(\mathrm{~d}, J=10.8 \mathrm{~Hz}, 3 \mathrm{H}), 3.76$ $(\mathrm{d}, J=10.8 \mathrm{~Hz}, 3 \mathrm{H}), 3.55-3.50(\mathrm{~m}, 1 \mathrm{H}), 3.29-3.23(\mathrm{~m}, 1 \mathrm{H}), 1.60-1.52(\mathrm{~m}, 2 \mathrm{H}), 0.86(\mathrm{t}, J=7.2$ $\mathrm{Hz}, 3 \mathrm{H}) .{ }^{31} \mathrm{P}$ NMR (161.9 MHz, $\left.\mathrm{CDCl}_{3}\right):$ 20.70. HRMS for $\mathrm{C}_{11} \mathrm{H}_{18} \mathrm{~N}_{3} \mathrm{NaO}_{4} \mathrm{P}^{+}$: calcd. $[\mathrm{M}+\mathrm{Na}]^{+}$: 310.0927, found: 310.0932. FTIR $\left(\mathrm{cm}^{-1}\right)$ : 3178, 1638, 1403, 1053, 833, 771.

Compound 40: Dimethyl (1-propyl-5-((E)-styryl)-4,5-dihydro-1H-1,2,3-triazol-4yl)phosphonate

Following the general procedure, treatment of cinnamaldehyde 10 (50 $\mathrm{mg}, 0.38 \mathrm{mmol}$ ) with n-propylamine 3a (34 mg, $0.57 \mathrm{mmol}$ ) and BOR (109 mg, $0.57 \mathrm{mmol}$ ) in $\mathrm{MeOH}(3 \mathrm{ml})$ at 


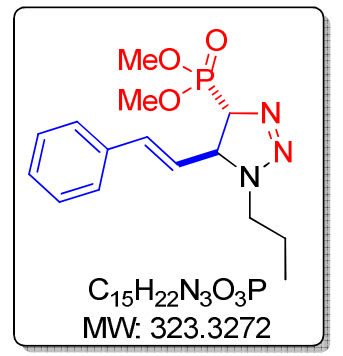

MW: 323.3272

room temperature for $6 \mathrm{~h}$ followed by column chromatography afforded the product 40 as a yellow liquid $\left(71 \mathrm{mg}, 58 \%\right.$ ). $\mathbf{R}_{\mathbf{f}}$ (Ethyl acetate/Hexane : 40/60) $=0.46 .{ }^{13} \mathrm{C}$ NMR $\left(100 \mathrm{MHz}, \delta \mathrm{ppm} / \mathrm{CDCl}_{3}\right)$ : $135.6(\mathrm{C}), 135.1(\mathrm{CH}), 128.8(\mathrm{CH}), 128.8(\mathrm{CH}), 128.6(\mathrm{CH}), 126.8(\mathrm{CH})$, $126.8(\mathrm{CH}), 125.3\left(\mathrm{~d}, J_{C-P}=11.7 \mathrm{~Hz}, \mathrm{CH}\right), 78.7\left(\mathrm{~d}, J_{C-P}=160.8 \mathrm{~Hz}, \mathrm{CH}\right)$, $62.3(\mathrm{CH}), 54.0\left(\mathrm{~d}, J_{C-P}=6.6 \mathrm{~Hz}, \mathrm{CH}_{3}\right), 53.4\left(\mathrm{~d}, J_{C-P}=6.7 \mathrm{~Hz}, \mathrm{CH}_{3}\right), 49.8$ $\left(\mathrm{CH}_{2}\right), 21.4\left(\mathrm{CH}_{2}\right), 11.4\left(\mathrm{CH}_{3}\right) .{ }^{1} \mathrm{H}$ NMR $\left(400 \mathrm{MHz}, \delta \mathrm{ppm} / \mathrm{CDCl}_{3}\right):$ 7.38$7.27(\mathrm{~m}, 5 \mathrm{H}), 6.64(\mathrm{~d}, J=15.6 \mathrm{~Hz}, 1 \mathrm{H}), 5.97(\mathrm{dd}, J=16.0,8.8 \mathrm{~Hz}, 1 \mathrm{H}), 4.46-4.27(\mathrm{~m}, 2 \mathrm{H}), 3.87$ $(\mathrm{d}, J=10.4 \mathrm{~Hz}, 3 \mathrm{H}), 3.81(\mathrm{~d}, J=10.8 \mathrm{~Hz}, 3 \mathrm{H}), 3.61-3.54(\mathrm{~m}, 1 \mathrm{H}), 3.47-3.40(\mathrm{~m}, 1 \mathrm{H}), 1.75-1.69$ $(\mathrm{m}, 2 \mathrm{H}), 0.95(\mathrm{t}, J=7.6 \mathrm{~Hz}, 3 \mathrm{H}) .{ }^{31} \mathrm{P}$ NMR $\left(161.9 \mathrm{MHz}, \mathrm{CDCl}_{3}\right)$ : 21.20. HRMS for $\mathrm{C}_{15} \mathrm{H}_{22} \mathrm{~N}_{3} \mathrm{NaO}_{3} \mathrm{P}^{+}$: calcd. [M+Na] $]^{+}: 346.1291$, found: 346.1281 . FTIR $\left(\mathrm{cm}^{-1}\right): 3391,3020,1638$, $1403,1217,1053,833,771,669$.

Compound 4p: Dimethyl (5-((E)-pent-1-en-1-yl)-1-propyl-4,5-dihydro-1H-1,2,3-triazol-4yl)phosphonate

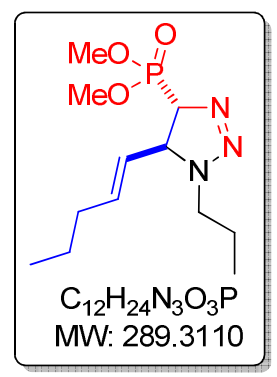

Following the general procedure, treatment of trans-1-hexen-2-al 1p (50 $\mathrm{mg}, 0.51 \mathrm{mmol}$ ) with $\mathrm{n}$-propylamine $3 \mathrm{a}(45 \mathrm{mg}, 0.76 \mathrm{mmol}$ ) and BOR (147 $\mathrm{mg}, 0.76 \mathrm{mmol}$ ) in $\mathrm{MeOH}(3 \mathrm{ml})$ at room temperature for $6 \mathrm{~h}$ followed by column chromatography afforded the product $4 p$ as a colorless liquid (78 $\mathrm{mg}, 53 \%) . \mathbf{R}_{\mathbf{f}}$ (Ethyl acetate/Hexane : 50/50) $=0.42 .{ }^{13} \mathrm{C} \mathrm{NMR}(100 \mathrm{MHz}, \delta$ $\left.\mathrm{ppm} / \mathrm{CDCl}_{3}\right): 137.3(\mathrm{CH}), 126.4\left(\mathrm{~d}, J_{C-P}=11.5 \mathrm{~Hz}, \mathrm{CH}\right), 78.4\left(\mathrm{~d}, J_{C-p}=161.2\right.$ $\mathrm{Hz}, \mathrm{CH}), 62.2(\mathrm{CH}), 54.0\left(\mathrm{~d}, J_{\mathrm{C}-\mathrm{p}}=6.6 \mathrm{~Hz}, \mathrm{CH}_{3}\right), 53.4\left(\mathrm{~d}, J_{\mathrm{C}-\mathrm{p}}=6.7 \mathrm{~Hz}, \mathrm{CH}_{3}\right)$, $49.5\left(\mathrm{CH}_{2}\right), 34.3\left(\mathrm{CH}_{2}\right), 22.1\left(\mathrm{CH}_{2}\right), 21.4\left(\mathrm{CH}_{2}\right), 13.6\left(\mathrm{CH}_{3}\right), 11.4\left(\mathrm{CH}_{3}\right) .{ }^{1} \mathrm{H}$ NMR $(400 \mathrm{MHz}, \delta$ ppm/ $\mathrm{CDCl}_{3}$ ): 5.79-5.72 (m, $\left.1 \mathrm{H}\right), 5.24$ (dd, $\left.J=15.2,8.4 \mathrm{~Hz}, 1 \mathrm{H}\right), 4.29$ (dd, $J=16.8,12.0 \mathrm{~Hz}$, $1 \mathrm{H}), 4.18-4.04(\mathrm{~m}, 1 \mathrm{H}), 3.85(\mathrm{~d}, J=10.8 \mathrm{~Hz}, 3 \mathrm{H}), 3.81(\mathrm{~d}, J=10.8 \mathrm{~Hz}, 3 \mathrm{H}), 3.56-3.49(\mathrm{~m}, 1 \mathrm{H})$, 3.41-3.34 (m, 1H), 2.06-2.01 (m, 2H), 1.71-167 (m, 2H), 1.43-1.37 (m, 2H), $0.94(\mathrm{t}, J=7.2 \mathrm{~Hz}$, $3 \mathrm{H}), 0.89(\mathrm{t}, J=7.6 \mathrm{~Hz}, 3 \mathrm{H}) .{ }^{31} \mathrm{P}$ NMR $\left(161.9 \mathrm{MHz}, \mathrm{CDCl}_{3}\right): 21.57$. HRMS for $\mathrm{C}_{12} \mathrm{H}_{24} \mathrm{~N}_{3} \mathrm{NaO}_{3} \mathrm{P}^{+}$: calcd. $[\mathrm{M}+\mathrm{Na}]^{+}:$312.1447, found: 312.1448. FTIR $\left(\mathrm{cm}^{-1}\right): 3391,3020,2400,1638,1403$, 1216, 1067, 929, 832, 778, 669.

\section{Compound 4q: Dimethyl (1-(tert-butyl)-5-((E)-styryl)-4,5-dihydro-1H-1,2,3-triazol-4-} yl)phosphonate

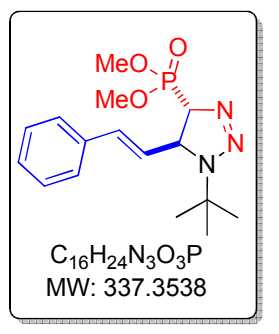

Following the general procedure, treatment of cinnamaldehyde 1q $(50 \mathrm{mg}$, $0.38 \mathrm{mmol}$ ) with tert-butylamine $3 \mathbf{b}(42 \mathrm{mg}, 0.57 \mathrm{mmol}$ ) and BOR (109 mg, $0.57 \mathrm{mmol}$ ) in $\mathrm{MeOH}(3 \mathrm{ml}$ ) at room temperature for $6 \mathrm{~h}$ followed by column chromatography afforded the product $\mathbf{4 q}$ as a white solid (86 $\mathbf{m g}$, 67\%). $\mathbf{M p}=132-134{ }^{\circ} \mathrm{C} . \mathbf{R}_{\mathbf{f}}$ (Ethyl acetate/Hexane : 40/60) $=0.40 .{ }^{13} \mathrm{C} \mathrm{NMR}$ (100 MHz, $\delta p p m / \mathrm{CDCl}_{3}$ ): $136.0(\mathrm{C}), 132.6(\mathrm{CH}), 129.2$ (d, $\left.J_{C-p}=13.5 \mathrm{~Hz}, \mathrm{CH}\right)$, $128.8(\mathrm{CH}), 128.8(\mathrm{CH}), 128.3(\mathrm{CH}), 126.6(\mathrm{CH}), 126.6(\mathrm{CH}), 79.9\left(\mathrm{~d}, J_{C-P}=156.3 \mathrm{~Hz}, \mathrm{CH}\right), 59.0$ $(\mathrm{CH}), 58.0(\mathrm{C}), 54.0\left(\mathrm{~d}, \mathrm{~J}_{\mathrm{C}-\mathrm{P}}=6.6 \mathrm{~Hz}, \mathrm{CH}_{3}\right), 53.5\left(\mathrm{~d}, \mathrm{~J}_{\mathrm{C}-\mathrm{P}}=6.7 \mathrm{~Hz}, \mathrm{CH}_{3}\right), 29.2\left(\mathrm{CH}_{3}\right), 29.2\left(\mathrm{CH}_{3}\right)$, 
$29.2\left(\mathrm{CH}_{3}\right) .{ }^{1} \mathrm{H}$ NMR $\left(400 \mathrm{MHz}, \delta \mathrm{ppm} / \mathrm{CDCl}_{3}\right)$ : 7.36-7.27 (m, 5H), $6.57(\mathrm{~d}, J=16.0 \mathrm{~Hz}, 1 \mathrm{H})$, 6.07 (dd, $J=16.0,8.4 \mathrm{~Hz}, 1 \mathrm{H}), 4.58-4.41(\mathrm{~m}, 2 \mathrm{H}), 3.85(\mathrm{~d}, J=10.8 \mathrm{~Hz}, 3 \mathrm{H}), 3.82(\mathrm{~d}, J=10.8$ $\mathrm{Hz}, 3 \mathrm{H}), 1.41$ (s, 9H). ${ }^{31} \mathrm{P}$ NMR (161.9 MHz, CDCl $)$ : 20.87. HRMS for $\mathrm{C}_{16} \mathrm{H}_{24} \mathrm{~N}_{3} \mathrm{NaO}_{3} \mathrm{P}^{+}$: calcd. $[\mathrm{M}+\mathrm{Na}]^{+}:$360.1447, found: 360.1459. FTIR $\left(\mathrm{cm}^{-1}\right): 3391,1651,14023,1051,833,772$.

\section{Compound 4r: Dimethyl (5-benzyl-1-propyl-4,5-dihydro-1H-1,2,3-triazol-4-yl)phosphonate}

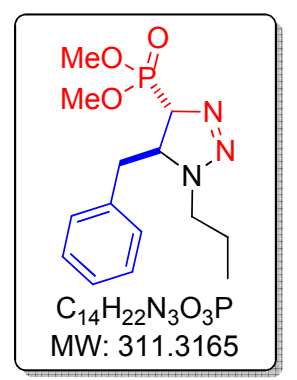

Following the general procedure, treatment of phenylacetaldehyde $\mathbf{1 r}$ (50 $\mathrm{mg}, 0.42 \mathrm{mmol}$ ) with $\mathrm{n}$-propylamine $3 \mathrm{a}(37 \mathrm{mg}, 0.62 \mathrm{mmol}$ ) and BOR (120 $\mathrm{mg}, 0.62 \mathrm{mmol}$ ) in $\mathrm{MeOH}(3 \mathrm{ml})$ at room temperature for $6 \mathrm{~h}$ followed by column chromatography afforded the product $4 r$ as a colorless liquid (52mg, 40\%). $\mathbf{R}_{\mathbf{f}}$ (Ethyl acetate/Hexane : 50/50) $=0.47 .{ }^{13} \mathbf{C}$ NMR (100 MHz, $\left.\delta \mathrm{ppm} / \mathrm{CDCl}_{3}\right): 135.7(\mathrm{C}), 129.7(\mathrm{CH}), 129.7(\mathrm{CH}), 128.9$ $(\mathrm{CH}), 128.9(\mathrm{CH}), 127.2(\mathrm{CH}), 76.2\left(\mathrm{~d}, J_{C-P}=158.4 \mathrm{~Hz}, \mathrm{CH}\right), 59.0(\mathrm{CH}), 53.8$ $\left(d, J_{C-P}=6.0 \mathrm{~Hz}, \mathrm{CH}_{3}\right), 53.3\left(\mathrm{~d}, J_{C-P}=6.0 \mathrm{~Hz}, \mathrm{CH}_{3}\right), 49.9\left(\mathrm{CH}_{2}\right), 38.4\left(\mathrm{~d}, J_{C-P}=13.7 \mathrm{~Hz}, \mathrm{CH}_{2}\right), 21.3$ $\left(\mathrm{CH}_{2}\right), 11.4\left(\mathrm{CH}_{3}\right) .{ }^{1} \mathrm{H}$ NMR $\left(400 \mathrm{MHz}, \delta \mathrm{ppm} / \mathrm{CDCl}_{3}\right): 7.33-7.25(\mathrm{~m}, 3 \mathrm{H}), 7.15(\mathrm{~d}, J=7.2 \mathrm{~Hz}$, $2 \mathrm{H}), 4.38(\mathrm{dd}, J=17.6,9.6 \mathrm{~Hz}, 1 \mathrm{H}), 4.10-4.02(\mathrm{~m}, 1 \mathrm{H}), 3.80-3.70(\mathrm{~m}, 1 \mathrm{H}), 3.73(\mathrm{~d}, J=10.4 \mathrm{~Hz}$, $3 \mathrm{H}), 3.67(\mathrm{~d}, J=10.8 \mathrm{~Hz}, 3 \mathrm{H}), 3.32-3.25(\mathrm{~m}, 1 \mathrm{H}), 2.94(\mathrm{dd}, J=14.0,5.6 \mathrm{~Hz}, 1 \mathrm{H}), 2.81(\mathrm{dd}, J=$ 14.0, $6.4 \mathrm{~Hz}, 1 \mathrm{H}), 1.75-1.64(\mathrm{~m}, 2 \mathrm{H}), 0.93(\mathrm{t}, J=7.6 \mathrm{~Hz}, 3 \mathrm{H}) .{ }^{31} \mathrm{P}$ NMR $\left(161.9 \mathrm{MHz}, \mathrm{CDCl}_{3}\right)$ : 20.88. HRMS for $\mathrm{C}_{14} \mathrm{H}_{22} \mathrm{~N}_{3} \mathrm{NaO}_{3} \mathrm{P}^{+}$: calcd. [M+Na] $]^{+}: 334.1291$, found: 334.1278 . FTIR $\left(\mathrm{cm}^{-1}\right)$ : 3390, 1650, 1403, 1067, 833, 771.

\section{Compound 4s:}

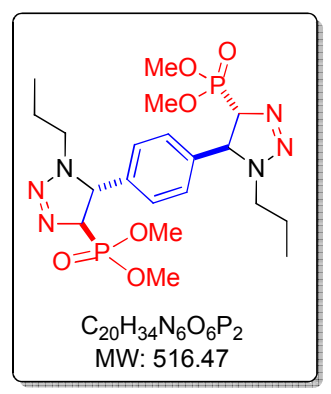

Following the general procedure, treatment of terephthaldehyde $1 \mathrm{~s}$ (50 $\mathrm{mg}, 0.37 \mathrm{mmol}$ ) with $\mathrm{n}$-propylamine $3 \mathrm{a}(88 \mathrm{mg}, 1.49 \mathrm{mmol})$ and BOR (286 $\mathrm{mg}, 1.49 \mathrm{mmol}$ ) in $\mathrm{MeOH}(3 \mathrm{ml}$ ) at room temperature for $6 \mathrm{~h}$ followed by column chromatography afforded the product $4 \mathrm{~s}$ as a white solid (150 $\mathrm{mg}, 78 \%)$. $\mathbf{R}_{\mathbf{f}}$ (acetone/dichloromethane : 30/70) = 0.41. $\mathrm{Mp}=140-142{ }^{\circ} \mathrm{C} .{ }^{13} \mathrm{C}$ NMR $\left(100 \mathrm{MHz}, \delta \mathrm{ppm} / \mathrm{CDCl}_{3}\right): 139.6\left(\mathrm{~d}, J_{C-P}\right.$ $=12.0 \mathrm{~Hz}, \mathrm{C}), 139.6\left(\mathrm{~d}, J_{C-P}=12.0 \mathrm{~Hz}, \mathrm{C}\right), 127.8(\mathrm{CH}), 127.8(\mathrm{CH}), 127.8$

$(\mathrm{CH}), 127.8(\mathrm{CH}), 82.0\left(\mathrm{~d}, J_{C-P}=157.7 \mathrm{~Hz}, \mathrm{CH}\right), 82.0\left(\mathrm{~d}, J_{C-P}=157.7 \mathrm{~Hz}, \mathrm{CH}\right), 62.1(\mathrm{CH}), 62.1$ $(\mathrm{CH}), 54.0\left(\mathrm{~d}, J_{C-P}=6.7 \mathrm{~Hz}, \mathrm{CH}_{3}\right), 54.0\left(\mathrm{~d}, J_{C-P}=6.7 \mathrm{~Hz}, \mathrm{CH}_{3}\right), 53.5\left(\mathrm{~d}, J_{C-P}=6.6 \mathrm{~Hz}, \mathrm{CH}_{3}\right), 53.5(\mathrm{~d}$, $\left.J_{C-P}=6.6 \mathrm{~Hz}, \mathrm{CH}_{3}\right), 49.9\left(\mathrm{CH}_{2}\right), 49.9\left(\mathrm{CH}_{2}\right), 21.2\left(\mathrm{CH}_{2}\right), 21.2\left(\mathrm{CH}_{2}\right), 11.3\left(\mathrm{CH}_{3}\right), 11.3\left(\mathrm{CH}_{3}\right) .{ }^{1} \mathbf{H}$ NMR $\left(400 \mathrm{MHz}, \delta \mathrm{ppm} / \mathrm{CDCl}_{3}\right): 7.21(\mathrm{~s}, 4 \mathrm{H}), 4.68(\mathrm{dd}, J=26.4,10.8 \mathrm{~Hz}, 2 \mathrm{H}), 4.52(\mathrm{dd}, J=$ 17.6, $10.8 \mathrm{~Hz}, 2 \mathrm{H}$ ), 3.84 (d, $J=10.8 \mathrm{~Hz}, 6 \mathrm{H}), 3.80$ (d, $J=10.8 \mathrm{~Hz}, 6 \mathrm{H}), 3.65-3.58(\mathrm{~m}, 2 \mathrm{H}), 3.29-$ $3.23(\mathrm{~m}, 2 \mathrm{H}), 1.63-1.58(\mathrm{~m}, 4 \mathrm{H}), 0.89(\mathrm{t}, J=7.6 \mathrm{~Hz}, 6 \mathrm{H}) .{ }^{31} \mathrm{P} \mathbf{N M R}\left(161.9 \mathrm{MHz}, \mathrm{CDCl}_{3}\right): 20.57$ (s, 2P). HRMS for $\mathrm{C}_{20} \mathrm{H}_{35} \mathrm{~N}_{6} \mathrm{O}_{6} \mathrm{P}_{2}{ }^{+}$: calcd. [M+H] $]^{+}: 517.2088$, found: 517.2101 . FTIR $\left(\mathrm{cm}^{-1}\right)$ : 3677, 3391, 3020, 2400, 1651, 1523, 1404, 1265, 1216, 1047, 929, 760, 669. 


\section{Compound 4t: Dimethyl (1-butyl-5-phenyl-4,5-dihydro-1H-1,2,3-triazol-4-yl)phosphonate}

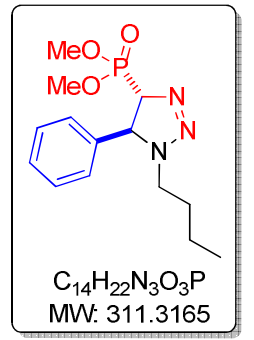

Following the general procedure, treatment of benzaldehyde $1 \mathrm{a}$ (50 $\mathrm{mg}$, $0.47 \mathrm{mmol}$ ) with $n$-butylamine $3 c(52 \mathrm{mg}, 0.71 \mathrm{mmol})$ and BOR (136 mg, $0.71 \mathrm{mmol})$ in $\mathrm{MeOH}(3 \mathrm{ml})$ at room temperature for $6 \mathrm{~h}$ followed by column chromatography afforded the product $\mathbf{4 t}$ as a colorless liquid (112 $\mathrm{mg}, 76 \%) . \mathbf{R}_{\mathbf{f}}$ (Ethyl acetate/Hexane : 50/50) $=0.40 .{ }^{13} \mathrm{C} \mathrm{NMR}(100 \mathrm{MHz}, \delta$ $\mathrm{ppm} / \mathrm{CDCl}_{3}$ ): $139.0\left(\mathrm{~d}, J_{C-P}=11.7 \mathrm{~Hz}, \mathrm{C}\right), 129.2(\mathrm{CH}), 129.2(\mathrm{CH}), 128.7(\mathrm{CH})$,

$127.1(\mathrm{CH}), 127.1(\mathrm{CH}), 81.9\left(\mathrm{~d}, J_{C-P}=158.9 \mathrm{~Hz}, \mathrm{CH}\right), 62.7(\mathrm{CH}), 54.0\left(\mathrm{~d}, J_{C-P}=6.4 \mathrm{~Hz}, \mathrm{CH}_{3}\right)$, $53.4\left(\mathrm{~d}, \mathrm{~J}_{\mathrm{C}-\mathrm{p}}=6.8 \mathrm{~Hz}, \mathrm{CH}_{3}\right), 47.8\left(\mathrm{CH}_{2}\right), 29.9\left(\mathrm{CH}_{2}\right) 19.9\left(\mathrm{CH}_{2}\right), 13.7\left(\mathrm{CH}_{3}\right) .{ }^{1} \mathrm{H}$ NMR $(400 \mathrm{MHz}, \delta$ $\left.\mathrm{ppm} / \mathrm{CDCl}_{3}\right):$ 7.37-7.31 (m, 3H), 7.22-7.21 (d, $\left.J=6.8 \mathrm{~Hz}, 2 \mathrm{H}\right), 4.70(\mathrm{dd}, J=26.0,11.2 \mathrm{~Hz}, 1 \mathrm{H})$, $4.55(\mathrm{dd}, J=17.2,11.2 \mathrm{~Hz}, 1 \mathrm{H}), 3.85(\mathrm{~d}, J=10.8 \mathrm{~Hz}, 3 \mathrm{H}), 3.78(\mathrm{~d}, J=10.8 \mathrm{~Hz}, 3 \mathrm{H}), 3.67-3.62$ $(\mathrm{m}, 1 \mathrm{H}), 3.32-3.25(\mathrm{~m}, 1 \mathrm{H}), 1.59-1.54(\mathrm{~m}, 2 \mathrm{H}), 1.33-1.27(\mathrm{~m}, 2 \mathrm{H}), 0.86(\mathrm{t}, J=7.2 \mathrm{~Hz}, 3 \mathrm{H}) .{ }^{31} \mathbf{P}$ NMR (161.9 MHz, CDCl $)$ : 20.79. HRMS for $\mathrm{C}_{14} \mathrm{H}_{22} \mathrm{~N}_{3} \mathrm{NaO}_{3} \mathrm{P}^{+}$: calcd. [M+Na] $]^{+}: 334.1291$, found: 334.1295. FTIR (cm ${ }^{-1}$ ): 3399, 3019, 1644, 1403, 1216, 1049, 929, 771, 669.

\section{Compound 4u: Dimethyl (1-octyl-5-phenyl-4,5-dihydro-1H-1,2,3-triazol-4-yl)phosphonate}

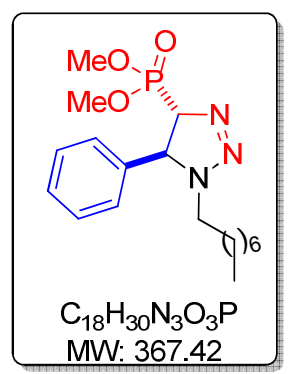

Following the general procedure, treatment of benzaldehyde $1 \mathrm{a}$ (50 $\mathrm{mg}$, $0.47 \mathrm{mmol}$ ) with $n$-octylamine $3 \mathrm{~d}(91 \mathrm{mg}, 0.71 \mathrm{mmol}$ ) and BOR (136 mg, $0.71 \mathrm{mmol}$ ) in $\mathrm{MeOH}(3 \mathrm{ml})$ at room temperature for $6 \mathrm{~h}$ followed by column chromatography afforded the product $\mathbf{4} \mathbf{u}$ as a colorless liquid (120 mg, 69\%). $\mathbf{R}_{\mathbf{f}}$ (Ethyl acetate/Hexane : 50/50) = 0.46. ${ }^{13} \mathrm{C}$ NMR (100 $\left.\mathrm{MHz}, \delta \mathrm{ppm} / \mathrm{CDCl}_{3}\right): 139.0\left(\mathrm{~d}, J_{C-p}=11.7 \mathrm{~Hz}, \mathrm{C}\right), 129.2(\mathrm{CH}), 129.2(\mathrm{CH})$, $128.7(\mathrm{CH}), 127.1(\mathrm{CH}), 127.1(\mathrm{CH}), 81.90\left(\mathrm{~d}, J_{C-P}=159.0 \mathrm{~Hz}, \mathrm{CH}\right), 62.7$ $(\mathrm{CH}), 54.0\left(\mathrm{~d}, J_{C-P}=6.7 \mathrm{~Hz}, \mathrm{CH}_{3}\right), 53.4\left(\mathrm{~d}, J_{C-P}=6.8 \mathrm{~Hz}, \mathrm{CH}_{3}\right), 48.1\left(\mathrm{CH}_{2}\right), 31.8\left(\mathrm{CH}_{2}\right), 29.2\left(\mathrm{CH}_{2}\right)$, $29.1\left(\mathrm{CH}_{2}\right), 27.8\left(\mathrm{CH}_{2}\right), 26.7\left(\mathrm{CH}_{2}\right), 22.7\left(\mathrm{CH}_{2}\right), 14.1\left(\mathrm{CH}_{3}\right) .{ }^{1} \mathrm{H} \mathbf{N M R}\left(400 \mathrm{MHz}, \delta \mathrm{ppm} / \mathrm{CDCl}_{3}\right)$ : 7.37-7.30 (m, 3H), 7.22-7.20 (m, 2H), 4.69 (dd, J = 26.0, $10.8 \mathrm{~Hz}, 1 \mathrm{H}), 4.55$ (dd, J = 17.2, 11.2 $\mathrm{Hz}, 1 \mathrm{H}), 3.84(\mathrm{~d}, J=10.8 \mathrm{~Hz}, 3 \mathrm{H}), 3.77(\mathrm{~d}, J=10.8 \mathrm{~Hz}, 3 \mathrm{H}), 3.64-3.59(\mathrm{~m}, 1 \mathrm{H}), 3.32-3.25(\mathrm{~m}$, $1 \mathrm{H}), 1.59-1.54(\mathrm{~m}, 2 \mathrm{H}), 1.27-1.21(\mathrm{~m}, 10 \mathrm{H}), 0.85(\mathrm{t}, J=6.8 \mathrm{~Hz}, 3 \mathrm{H}) .{ }^{31} \mathbf{P}$ NMR $(161.9 \mathrm{MHz}$, $\mathrm{CDCl}_{3}$ ): 20.91. HRMS for $\mathrm{C}_{18} \mathrm{H}_{30} \mathrm{~N}_{3} \mathrm{NaO}_{3} \mathrm{P}^{+}$: calcd. [M+Na] ${ }^{+}: 390.1917$, found: 390.1922. FTIR $\left(\mathrm{cm}^{-1}\right)$ : 3399, 1637, 1403, 1219, 1053, 832, 772.

\section{Compound 4v: Dimethyl (1-nonyl-5-phenyl-4,5-dihydro-1H-1,2,3-triazol-4-yl)phosphonate}

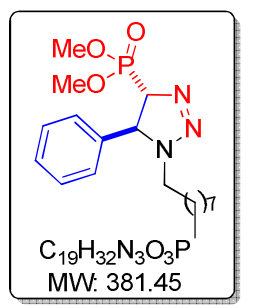

Following the general procedure, treatment of benzaldehyde $1 \mathrm{a}(50 \mathrm{mg}$, $0.47 \mathrm{mmol}$ ) with $n$-nonylamine 3 e $(101 \mathrm{mg}, 0.71 \mathrm{mmol})$ and BOR (136 mg, $0.71 \mathrm{mmol}$ ) in $\mathrm{MeOH}(3 \mathrm{ml}$ ) at room temperature for $6 \mathrm{~h}$ followed by column chromatography afforded the product $\mathbf{4} \mathbf{v}$ as a colorless liquid (117 $\mathrm{mg}, 65 \%) . \mathbf{R}_{\mathbf{f}}$ (Ethyl acetate/Hexane : 50/50) $=0.42 .{ }^{13} \mathrm{C} \mathrm{NMR}(100 \mathrm{MHz}, \delta$ $\left.\mathrm{ppm} / \mathrm{CDCl}_{3}\right): 139.0\left(\mathrm{~d}, J_{\mathrm{C}-\mathrm{p}}=11.6 \mathrm{~Hz}, \mathrm{C}\right), 129.3(\mathrm{CH}), 129.3(\mathrm{CH}), 128.7(\mathrm{CH})$, $127.1(\mathrm{CH}), 127.1(\mathrm{CH}), 82.0\left(\mathrm{~d}, J_{C-P}=159.1 \mathrm{~Hz}, \mathrm{CH}\right), 62.8(\mathrm{CH}), 54.0\left(\mathrm{~d}, J_{C-P}=6.5 \mathrm{~Hz}, \mathrm{CH}_{3}\right)$, $53.5\left(\mathrm{~d}, J_{C-p}=6.6 \mathrm{~Hz}, \mathrm{CH}_{3}\right), 48.2\left(\mathrm{CH}_{2}\right), 32.0\left(\mathrm{CH}_{2}\right), 29.6\left(\mathrm{CH}_{2}\right), 29.3\left(\mathrm{CH}_{2}\right), 29.3\left(\mathrm{CH}_{2}\right), 27.9$ 
$\left(\mathrm{CH}_{2}\right), 26.8\left(\mathrm{CH}_{2}\right), 22.8\left(\mathrm{CH}_{2}\right), 14.2\left(\mathrm{CH}_{3}\right) .{ }^{1} \mathrm{H}$ NMR $\left(400 \mathrm{MHz}, \delta \mathrm{ppm} / \mathrm{CDCl}_{3}\right):$ 7.39-7.30 $(\mathrm{m}, 3 \mathrm{H})$, 7.24-7.22 (m, 2H), $4.70(\mathrm{dd}, J=26.0,11.2 \mathrm{~Hz}, 1 \mathrm{H}), 4.56(\mathrm{dd}, J=17.2,10.8 \mathrm{~Hz}, 1 \mathrm{H}), 3.85$ (d, $J=$ $10.8 \mathrm{~Hz}, 3 \mathrm{H}), 3.79(\mathrm{~d}, J=10.8 \mathrm{~Hz}, 3 \mathrm{H}), 3.68-3.60(\mathrm{~m}, 1 \mathrm{H}), 3.33-3.26(\mathrm{~m}, 1 \mathrm{H}), 1.61-1.56(\mathrm{~m}$, $2 \mathrm{H}), 1.29-1.23(\mathrm{~m}, 12 \mathrm{H}), 0.87(\mathrm{t}, J=6.8 \mathrm{~Hz}, 3 \mathrm{H}) .{ }^{31} \mathrm{P} \mathbf{N M R}\left(161.9 \mathrm{MHz}, \mathrm{CDCl}_{3}\right): 20.92$. HRMS for $\mathrm{C}_{19} \mathrm{H}_{32} \mathrm{~N}_{3} \mathrm{NaO}_{3} \mathrm{P}^{+}$: calcd. [M+Na] $]^{+}: 404.2073$, found: 404.2073. FTIR (cm $\left.{ }^{-1}\right): 3391,2927,1625$, $1403,1219,1054,1054,832,772$.

\section{Compound 4w: Dimethyl (1-decyl-5-phenyl-4,5-dihydro-1H-1,2,3-triazol-4-yl)phosphonate}

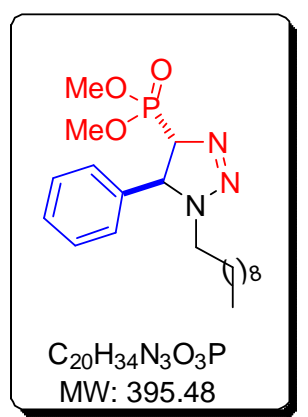

Following the general procedure, treatment of benzaldehyde $1 \mathrm{a}(50 \mathrm{mg}$, $0.47 \mathrm{mmol}$ ) with $n$-decylamine $\mathbf{3 f}$ (111 $\mathrm{mg}, 0.71 \mathrm{mmol}$ ) and BOR (136 mg, $0.71 \mathrm{mmol}$ ) in $\mathrm{MeOH}(3 \mathrm{ml})$ at room temperature for $6 \mathrm{~h}$ followed by column chromatography afforded the product $\mathbf{4 w}$ as a colorless liquid (114 $\mathbf{m g}, 61 \%)$. $\mathbf{R}_{\mathbf{f}}$ (Ethyl acetate/Hexane : 50/50) =0.45. ${ }^{13} \mathbf{C} \mathbf{~ N M R}(100$ $\left.\mathrm{MHz}, \delta \mathrm{ppm} / \mathrm{CDCl}_{3}\right): 139.0\left(\mathrm{~d}, J_{\mathrm{C}-\mathrm{P}}=11.7 \mathrm{~Hz}, \mathrm{C}\right), 129.2(\mathrm{CH}), 129.2(\mathrm{CH})$, $128.7(\mathrm{CH}), 127.1(\mathrm{CH}), 127.1(\mathrm{CH}), 81.9\left(\mathrm{~d}, J_{C-p}=159.1 \mathrm{~Hz}, \mathrm{CH}\right), 62.8(\mathrm{CH})$, $54.0\left(\mathrm{~d}, J_{C-P}=6.7 \mathrm{~Hz}, \mathrm{CH}_{3}\right), 53.5\left(\mathrm{~d}, J_{C-p}=6.7 \mathrm{~Hz}, \mathrm{CH}_{3}\right), 48.2\left(\mathrm{CH}_{2}\right), 32.0\left(\mathrm{CH}_{2}\right) 29.6\left(\mathrm{CH}_{2}\right), 29.6$ $\left(\mathrm{CH}_{2}\right), 29.4\left(\mathrm{CH}_{2}\right), 29.2\left(\mathrm{CH}_{2}\right), 27.8\left(\mathrm{CH}_{2}\right), 26.7\left(\mathrm{CH}_{2}\right), 22.7\left(\mathrm{CH}_{2}\right), 14.2\left(\mathrm{CH}_{3}\right) .{ }^{1} \mathbf{H}$ NMR (400 $\left.\mathrm{MHz}, \delta \mathrm{ppm} / \mathrm{CDCl}_{3}\right): 7.37-7.29(\mathrm{~m}, 3 \mathrm{H}), 7.23-7.20(\mathrm{~m}, 2 \mathrm{H}), 4.69(\mathrm{dd}, J=26.0,10.8 \mathrm{~Hz}, 1 \mathrm{H})$, 4.55 (dd, $J=17.2,11.2 \mathrm{~Hz}, 1 \mathrm{H}$ ), $3.84(\mathrm{~d}, J=10.8 \mathrm{~Hz}, 3 \mathrm{H}), 3.78(\mathrm{~d}, J=10.8 \mathrm{~Hz}, 3 \mathrm{H}$ ), 3.65-3.61 $(\mathrm{m}, 1 \mathrm{H}), 3.32-3.25(\mathrm{~m}, 1 \mathrm{H}), 1.60-1.54(\mathrm{~m}, 2 \mathrm{H}), 1.27-1.22(\mathrm{~m}, 14 \mathrm{H}), 0.86(\mathrm{t}, J=6.8 \mathrm{~Hz}, 3 \mathrm{H}) .{ }^{31} \mathbf{P}$ NMR (161.9 MHz, CDCl 3 ): 20.92. HRMS for $\mathrm{C}_{20} \mathrm{H}_{34} \mathrm{~N}_{3} \mathrm{NaO}_{3} \mathrm{P}^{+}$: calcd. [M+Na] $]^{+}:$418.2230, found: 418.2251. FTIR (cm $\left.{ }^{-1}\right): 3391,3020,2929,2400,1625,1523,1404,1216,1054,929$, $758,669,627$.

\section{Compound 4x: Dimethyl (1-(tert-butyl)-5-phenyl-4,5-dihydro-1H-1,2,3-triazol-4- yl)phosphonate}

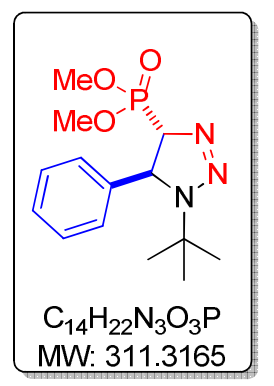

Following the general procedure, treatment of benzaldehyde $1 \mathrm{a}(50 \mathrm{mg}$, $0.47 \mathrm{mmol}$ ) with tert-butylamine $\mathbf{3 b}(52 \mathrm{mg}, 0.71 \mathrm{mmol}$ ) and BOR (136 mg, $0.71 \mathrm{mmol}$ ) in $\mathrm{MeOH}(3 \mathrm{ml})$ at room temperature for $6 \mathrm{~h}$ followed by column chromatography afforded the product $\mathbf{4 x}$ as a colorless liquid (92 $\mathrm{mg}, 63 \%)$. $\mathbf{R}_{\mathbf{f}}$ (Ethyl acetate/Hexane : 50/50) $=0.38 .{ }^{13} \mathbf{C ~ N M R ~}(100 \mathrm{MHz}, \delta$ $\left.\mathrm{ppm} / \mathrm{CDCl}_{3}\right): 143.4\left(\mathrm{~d}, J_{C-p}=12.2 \mathrm{~Hz}, \mathrm{C}\right), 129.1(\mathrm{CH}), 129.1(\mathrm{CH}), 128.0(\mathrm{CH})$, $126.6(\mathrm{CH}), 126.6(\mathrm{CH}), 83.6\left(\mathrm{~d}, J_{C-P}=155.9 \mathrm{~Hz}, \mathrm{CH}\right), 59.5(\mathrm{CH}), 58.2(\mathrm{C})$, $53.9\left(\mathrm{~d}, J_{C-P}=6.8 \mathrm{~Hz}, \mathrm{CH}_{3}\right), 53.5\left(\mathrm{~d}, J_{\mathrm{C}-\mathrm{P}}=6.7 \mathrm{~Hz}, \mathrm{CH}_{3}\right), 29.1\left(\mathrm{CH}_{3}\right), 29.1\left(\mathrm{CH}_{3}\right), 29.1\left(\mathrm{CH}_{3}\right) .{ }^{1} \mathrm{H}$ NMR $\left(400 \mathrm{MHz}, \delta \mathrm{ppm} / \mathrm{CDCl}_{3}\right): 7.34-7.27(\mathrm{~m}, 3 \mathrm{H}), 7.22-7.20(\mathrm{~m}, 2 \mathrm{H}), 4.77(\mathrm{dd}, J=28.0,10.0$ $\mathrm{Hz}, 1 \mathrm{H}), 4.50$ (dd, $J=17.6,10.0 \mathrm{~Hz}, 1 \mathrm{H}), 3.84$ (d, $J=10.8 \mathrm{~Hz}, 3 \mathrm{H}), 3.80$ (d, $J=10.8 \mathrm{~Hz}, 3 \mathrm{H}$ ), $1.28(\mathrm{~s}, 9 \mathrm{H}) .{ }^{31} \mathrm{P}$ NMR $\left(161.9 \mathrm{MHz}, \mathrm{CDCl}_{3}\right): 20.58$. HRMS for $\mathrm{C}_{14} \mathrm{H}_{22} \mathrm{~N}_{3} \mathrm{NaO}_{3} \mathrm{P}^{+}$: calcd. $[\mathrm{M}+\mathrm{Na}]^{+}$: 334.1296, found: 334.1287. FTIR ( $\left.\mathrm{cm}^{-1}\right)$ : 3400, 3019, 2400, 1633, 1403, 1216, 1059, 933, $831,770,669$. 


\section{Compound 4y: Dimethyl (1-allyl-5-phenyl-4,5-dihydro-1H-1,2,3-triazol-4-yl)phosphonate}

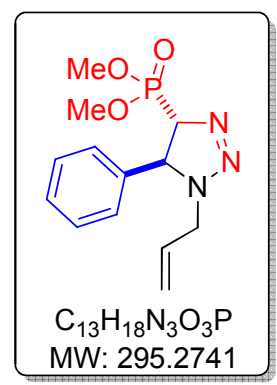

Following the general procedure, treatment of benzaldehyde $1 \mathrm{a}$ (50 mg, $0.47 \mathrm{mmol})$ with allylamine $3 \mathrm{~g}(40 \mathrm{mg}, 0.71 \mathrm{mmol})$ in the presence of $\mathrm{K}_{2} \mathrm{CO}_{3}$ (130 mg, $0.94 \mathrm{mmol}$ ) and $\mathrm{BOR}(136 \mathrm{mg}, 0.71 \mathrm{mmol})$ in $\mathrm{MeOH}(3 \mathrm{ml})$ at room temperature for $6 \mathrm{~h}$ followed by column chromatography afforded the product $4 \mathbf{y}$ as a yellow liquid $(69 \mathrm{mg}, 50 \%)$. $\mathbf{R}_{\mathbf{f}}$ (Ethyl acetate/Hexane : 50/50) $=0.45 .{ }^{13} \mathrm{C} \mathrm{NMR}\left(100 \mathrm{MHz}, \delta \mathrm{ppm} / \mathrm{CDCl}_{3}\right): 138.7$ $\left(d, J_{C-P}=10.0 \mathrm{~Hz}, \mathrm{C}\right), 132.0(\mathrm{CH}), 129.3(\mathrm{CH}), 129.3(\mathrm{CH}), 128.8(\mathrm{CH}), 127.3$ $(\mathrm{CH}), 127.3(\mathrm{CH}), 119.5\left(\mathrm{CH}_{2}\right), 82.4\left(\mathrm{~d}, J_{C-P}=159.5 \mathrm{~Hz}, \mathrm{CH}\right), 61.8(\mathrm{CH}), 54.1\left(\mathrm{~d}, J_{C-P}=6.7 \mathrm{~Hz}\right.$, $\left.\mathrm{CH}_{3}\right), 53.5\left(\mathrm{~d}, J_{C-p}=6.7 \mathrm{~Hz}, \mathrm{CH}_{3}\right), 50.6\left(\mathrm{CH}_{2}\right) .{ }^{1} \mathrm{H}$ NMR $\left(400 \mathrm{MHz}, \delta \mathrm{ppm} / \mathrm{CDCl}_{3}\right):$ 7.38-7.32 (m, $3 \mathrm{H}), 7.23-7.21(\mathrm{~m}, 2 \mathrm{H}), 5.82-5.73(\mathrm{~m}, 1 \mathrm{H}), 5.18(\mathrm{dd}, J=21.6,10.0 \mathrm{~Hz}, 2 \mathrm{H}), 4.76-4.49(\mathrm{~m}, 3 \mathrm{H})$, $3.86(\mathrm{~d}, J=10.8 \mathrm{~Hz}, 3 \mathrm{H}), 3.79(\mathrm{~d}, J=10.8 \mathrm{~Hz}, 3 \mathrm{H}), 3.82-3.64(\mathrm{~m}, 1 \mathrm{H}) .{ }^{31} \mathbf{P}$ NMR $(161.9 \mathrm{MHz}$, $\mathrm{CDCl}_{3}$ ): 20.71. HRMS for $\mathrm{C}_{13} \mathrm{H}_{18} \mathrm{~N}_{3} \mathrm{NaO}_{3} \mathrm{P}^{+}$: calcd. [M+Na] $]^{+}$318.0978, found: 318.0979. FTIR $\left(\mathrm{cm}^{-1}\right): 3684,3020,2927,2400,1625,1523,1475,1420,1216,1052,929,849,758,669$, 627.

Compound 4z: Dimethyl (1-isopropyl-5-phenyl-4,5-dihydro-1H-1,2,3-triazol-4yl)phosphonate

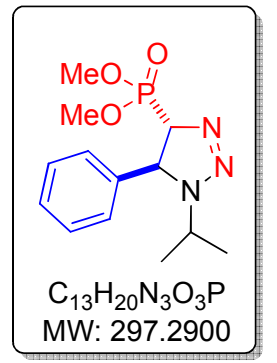

Following the general procedure, treatment of benzaldehyde $1 \mathrm{a}(50 \mathrm{mg}$, $0.47 \mathrm{mmol}$ ) with 2-aminopropane $3 \mathrm{~h}(42 \mathrm{mg}, 0.71 \mathrm{mmol}$ ) and BOR (136 $\mathrm{mg}, 0.71 \mathrm{mmol})$ in $\mathrm{MeOH}(3 \mathrm{ml})$ at room temperature for $6 \mathrm{~h}$ followed by column chromatography afforded the product $\mathbf{4 z}$ as a colorless liquid (91 mg, 65\%). $\mathbf{R}_{\mathbf{f}}$ (Ethyl acetate/Hexane : 50/50) $=0.35 .{ }^{13} \mathrm{C} \mathrm{NMR} \mathrm{(100} \mathrm{MHz,}$ $\left.\delta \mathrm{ppm} / \mathrm{CDCl}_{3}\right): 140.12\left(\mathrm{~d}, J_{C-p}=10.1 \mathrm{~Hz}, \mathrm{C}\right), 129.1(\mathrm{CH}), 129.1(\mathrm{CH}), 128.5$ $(\mathrm{CH}), 127.0(\mathrm{CH}), 127.0(\mathrm{CH}), 81.9\left(\mathrm{~d}, J_{C-P}=159.4 \mathrm{~Hz}, \mathrm{CH}\right), 61.4(\mathrm{CH}), 54.0(\mathrm{~d}$, $\left.J_{C-p}=6.5 \mathrm{~Hz}, \mathrm{CH}_{3}\right), 53.4\left(\mathrm{~d}, J_{C-P}=6.7 \mathrm{~Hz}, \mathrm{CH}_{3}\right), 50.5(\mathrm{CH}), 21.5\left(\mathrm{CH}_{3}\right), 20.8\left(\mathrm{CH}_{3}\right) .{ }^{1} \mathrm{H}$ NMR (400 $\left.\mathrm{MHz}, \delta \mathrm{ppm} / \mathrm{CDCl}_{3}\right): 7.24-7.14(\mathrm{~m}, 5 \mathrm{H}), 4.30(\mathrm{dd}, J=26.8,11.6 \mathrm{~Hz}, 1 \mathrm{H}), 4.40(\mathrm{dd}, J=17.2$, $11.2 \mathrm{~Hz}, 1 \mathrm{H}), 3.76(\mathrm{~d}, J=10.8 \mathrm{~Hz}, 3 \mathrm{H}), 3.69(\mathrm{~d}, J=10.8 \mathrm{~Hz}, 3 \mathrm{H}), 3.63-3.57(\mathrm{~m}, 1 \mathrm{H}), 1.31(\mathrm{~d}, J=$ $6.4 \mathrm{~Hz}, 3 \mathrm{H}), 1.07$ (d, $J=6.8 \mathrm{~Hz}, 3 \mathrm{H}) .{ }^{31} \mathrm{P}$ NMR (161.9 MHz, $\mathrm{CDCl}_{3}$ ): 20.96. HRMS for $\mathrm{C}_{13} \mathrm{H}_{21} \mathrm{~N}_{3} \mathrm{O}_{3} \mathrm{P}^{+}$: calcd. [M+H] $]^{+}: 298.1315$, found: 298.1317. FTIR $\left(\mathrm{cm}^{-1}\right): 3400,3019,2400$, $1645,1401,1216,1051,928,770,669,561$.

\section{Compound 4aa: Dimethyl (1-benzyl-5-phenyl-4,5-dihydro-1H-1,2,3-triazol-4- yl)phosphonate}

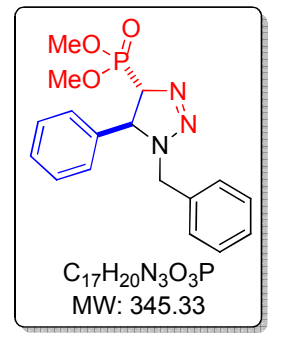

Following the general procedure, treatment of benzaldehyde $1 \mathrm{a}(50 \mathrm{mg}$, $0.47 \mathrm{mmol}$ ) with benzylamine $\mathbf{3 i}(76 \mathrm{mg}, 0.71 \mathrm{mmol})$ and BOR (136 $\mathrm{mg}$, $0.71 \mathrm{mmol})$ in $\mathrm{MeOH}(3 \mathrm{ml})$ at room temperature for $6 \mathrm{~h}$ followed by column chromatography afforded the product 4 aa as a colorless liquid (95 $\mathrm{mg}, 59 \%) . \mathbf{R}_{\mathbf{f}}$ (Ethyl acetate/Hexane : 50/50) $=0.45 .{ }^{13} \mathrm{C} \mathbf{N M R}(100 \mathrm{MHz}, \delta$ $\left.\mathrm{ppm} / \mathrm{CDCl}_{3}\right): 138.4(\mathrm{C}), 135.0(\mathrm{C}), 129.3(\mathrm{CH}), 129.3(\mathrm{CH}), 128.8(\mathrm{CH}), 128.8$ 
$(\mathrm{CH}), 128.7(\mathrm{CH}), 128.7(\mathrm{CH}), 128.2(\mathrm{CH}), 128.0(\mathrm{CH}), 127.3(\mathrm{CH}), 127.3(\mathrm{CH}), 82.3\left(\mathrm{~d}, J_{C-p}=\right.$ $160.0 \mathrm{~Hz}, \mathrm{CH}), 61.1(\mathrm{CH}), 53.8\left(\mathrm{~d}, J_{C-P}=6.7 \mathrm{~Hz}, \mathrm{CH}_{3}\right), 53.7\left(\mathrm{~d}, J_{C-P}=7.0 \mathrm{~Hz}, \mathrm{CH}_{3}\right), 51.8\left(\mathrm{CH}_{2}\right) .{ }^{1} \mathrm{H}$ NMR (400 MHz, $\left.\delta p p m / \mathrm{CDCl}_{3}\right): 7.36-7.30(\mathrm{~m}, 7 \mathrm{H}), 7.19-7.15(\mathrm{~m}, 3 \mathrm{H}), 5.24(\mathrm{~d}, J=15.2 \mathrm{~Hz}, 1 \mathrm{H})$, 4.64-4.43 (m, $2 \mathrm{H}), 4.19$ (d, $J=15.2 \mathrm{~Hz}, 1 \mathrm{H}), 3.74(\mathrm{~d}, J=10.9 \mathrm{~Hz}, 3 \mathrm{H}), 3.67$ (d, $J=10.7 \mathrm{~Hz}, 3 \mathrm{H})$.

${ }^{31} \mathrm{P}$ NMR (161.9 MHz, $\mathrm{CDCl}_{3}$ ): 20.33. HRMS for $\mathrm{C}_{17} \mathrm{H}_{20} \mathrm{~N}_{3} \mathrm{NaO}_{3} \mathrm{P}+$ : calcd. $[\mathrm{M}+\mathrm{Na}]^{+}:$368.1134, found: 368.1111. FTIR ( $\left.\mathrm{cm}^{-1}\right)$ : 3391, 3019, 2400, 1645, 1403, 1265, 1217, 1048, 927, 772, 669.

Compound 4ab: Dimethyl (1-phenethyl-5-phenyl-4,5-dihydro-1H-1,2,3-triazol-4yl)phosphonate

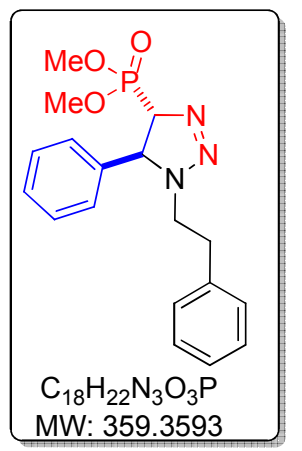

Following the general procedure, treatment of benzaldehyde $1 \mathrm{a}(50 \mathrm{mg}$, $0.47 \mathrm{mmol}$ ) with 2-phenylethylamine $3 \mathbf{j}(86 \mathrm{mg}, 0.71 \mathrm{mmol})$ and BOR (136 $\mathrm{mg}, 0.71 \mathrm{mmol}$ ) in $\mathrm{MeOH}(3 \mathrm{ml}$ ) at room temperature for $6 \mathrm{~h}$ followed by column chromatography afforded the product $4 \mathbf{a b}$ as a colorless liquid (108 $\mathrm{mg}, 64 \%$ ). $\mathbf{R}_{\mathbf{f}}$ (Ethyl acetate/Hexane : 50/50) $=0.46$. ${ }^{13} \mathrm{C}$ NMR (100 MHz, $\left.\delta \mathrm{ppm} / \mathrm{CDCl}_{3}\right): 139.7$ (C), $138.5(\mathrm{C}), 129.3(\mathrm{CH}), 129.3$ $(\mathrm{CH}), 128.9(\mathrm{CH}), 128.9(\mathrm{CH}), 128.8(\mathrm{CH}), 128.7(\mathrm{CH}), 128.7(\mathrm{CH}), 127.2$ $(\mathrm{CH}), 127.2(\mathrm{CH}), 126.6(\mathrm{CH}), 82.2\left(\mathrm{~d}, J_{C-P}=159.3 \mathrm{~Hz}, \mathrm{CH}\right), 63.1(\mathrm{CH}), 54.0$ $\left(d, J_{C-P}=6.5 \mathrm{~Hz}, \mathrm{CH}_{3}\right), 53.5\left(\mathrm{~d}, J_{C-P}=6.6 \mathrm{~Hz}, \mathrm{CH}_{3}\right), 49.5\left(\mathrm{CH}_{2}\right), 34.7\left(\mathrm{CH}_{2}\right) .{ }^{1} \mathrm{H} \mathrm{NMR}(400 \mathrm{MHz}, \delta$ $\left.\mathrm{ppm} / \mathrm{CDCl}_{3}\right)$ : 7.34-7.20 (m, 6H), 7.14-7.11 (m, 4H), 4.67 (dd, J = 25.6, $\left.11.2 \mathrm{~Hz}, 1 \mathrm{H}\right), 4.56$ (dd, $J$ $=17.2,11.2 \mathrm{~Hz}, 1 \mathrm{H}), 3.89-3.85(\mathrm{~m}, 1 \mathrm{H}), 3.82(\mathrm{~d}, J=10.8 \mathrm{~Hz}, 3 \mathrm{H}), 3.77(\mathrm{~d}, J=10.8 \mathrm{~Hz}, 3 \mathrm{H})$, 3.57-3.51 (m, 1H), 3.00-2.91 (m, 2H). ${ }^{31} \mathbf{P}$ NMR (161.9 MHz, $\left.\mathrm{CDCl}_{3}\right)$ : 20.74. HRMS for $\mathrm{C}_{18} \mathrm{H}_{22} \mathrm{~N}_{3} \mathrm{NaO}_{3} \mathrm{P}^{+}$: calcd. [M+Na] $]^{+}: 382.1291$, found: 382.1291. FTIR (cm $\left.{ }^{-1}\right): 3378,3019,2399$, $1650,1403,1265,1217,1049,928,771,669$.

\section{Compound 4ac: Dimethyl (1-cyclohexyl-5-phenyl-4,5-dihydro-1H-1,2,3-triazol-4- yl)phosphonate}

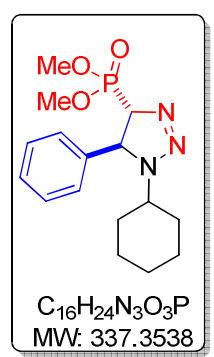

Following the general procedure, treatment of benzaldehyde 1a $(50 \mathrm{mg}, 0.47$ $\mathrm{mmol}$ ) with cyclohexylamine $3 \mathbf{k}(70 \mathrm{mg}, 0.71 \mathrm{mmol}$ ) and BOR (136 mg, 0.71 $\mathrm{mmol})$ in $\mathrm{MeOH}(3 \mathrm{ml})$ at room temperature for $6 \mathrm{~h}$ followed by column chromatography afforded the product $\mathbf{4 c}$ as a colorless liquid (91 $\mathrm{mg}, 57 \%$ ). $\mathbf{R}_{\mathbf{f}}$ (Ethyl acetate/Hexane : 50/50) =0.42. ${ }^{13} \mathrm{C} \mathrm{NMR}\left(100 \mathrm{MHz}, \delta \mathrm{ppm} / \mathrm{CDCl}_{3}\right)$ : $140.4\left(\mathrm{~d}, J_{C-P}=11.8 \mathrm{~Hz}, \mathrm{C}\right), 129.1(\mathrm{CH}), 129.1(\mathrm{CH}), 128.4(\mathrm{CH}), 126.9(\mathrm{CH})$, $126.9(\mathrm{CH}), 81.8\left(\mathrm{~d}, J_{C-P}=158.3 \mathrm{~Hz}, \mathrm{CH}\right), 61.1(\mathrm{CH}), 58.0(\mathrm{CH}), 54.0\left(\mathrm{~d}, J_{C-p}=6.5\right.$ $\left.\mathrm{Hz}, \mathrm{CH}_{3}\right), 53.4\left(\mathrm{~d}, J_{C-P}=6.7 \mathrm{~Hz}, \mathrm{CH}_{3}\right), 31.9\left(\mathrm{CH}_{2}\right), 31.5\left(\mathrm{CH}_{2}\right), 25.5\left(\mathrm{CH}_{2}\right), 25.5\left(\mathrm{CH}_{2}\right), 25.2\left(\mathrm{CH}_{2}\right)$. ${ }^{1} \mathrm{H}$ NMR $\left(400 \mathrm{MHz}, \delta \mathrm{ppm} / \mathrm{CDCl}_{3}\right): 7.35-7.27(\mathrm{~m}, 3 \mathrm{H}), 7.21(\mathrm{~d}, J=6.8 \mathrm{~Hz}, 2 \mathrm{H}), 4.76(\mathrm{dd}, J=$ 27.6, 11.2 Hz, 1H), 4.47 (dd, J=17.6, $11.2 \mathrm{~Hz}, 1 \mathrm{H}$ ), 3.83 (d, $J=10.8 \mathrm{~Hz}, 3 \mathrm{H}$ ), 3.77 (d, J = 10.8 $\mathrm{Hz}, 3 \mathrm{H}), 3.33-3.26(\mathrm{~m}, 1 \mathrm{H}), 1.93-1.70(\mathrm{~m}, 5 \mathrm{H}), 1.59-1.58(\mathrm{~m}, 1 \mathrm{H}), 1.25-1.14(\mathrm{~m}, 4 \mathrm{H}) .{ }^{31} \mathbf{P}$ NMR (161.9 MHz, CDCl 3 ): 20.90. HRMS for $\mathrm{C}_{16} \mathrm{H}_{24} \mathrm{~N}_{3} \mathrm{NaO}_{3} \mathrm{P}^{+}$: calcd. [M+Na] $]^{+}$:360.1447, found: 360.1443. FTIR (cm $\left.{ }^{-1}\right): 3400,3020,2400,1645,1523,1404,1216,1052,929,758,669,627$. 
Compound 4ad: Dimethyl (1-((3R,5R,7R)-adamantan-1-yl)-5-phenyl-4,5-dihydro-1H-1,2,3triazol-4-yl)phosphonate

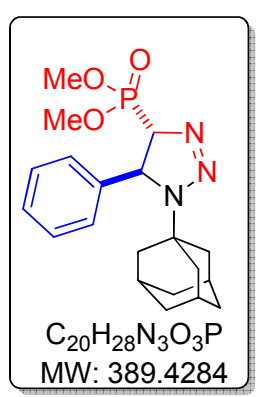

Following the general procedure, treatment of benzaldehyde $1 \mathrm{a}(50 \mathrm{mg}$, $0.47 \mathrm{mmol}$ ) with 1-adamantylamine 31 (107 $\mathrm{mg}, 0.71 \mathrm{mmol}$ ) and BOR (136 $\mathrm{mg}, 0.71 \mathrm{mmol})$ in $\mathrm{MeOH}(3 \mathrm{ml})$ at room temperature for $6 \mathrm{~h}$ followed by column chromatography afforded the product $4 \mathrm{ad}$ as a colorless liquid (119 mg, 65\%). $\mathbf{R}_{\mathbf{f}}$ (Ethyl acetate/Hexane : 50/50) = 0.45. ${ }^{13} \mathbf{C}$ NMR (100 $\left.\mathrm{MHz}, \delta \mathrm{ppm} / \mathrm{CDCl}_{3}\right): 143.6\left(\mathrm{~d}, J_{C-P}=13.1 \mathrm{~Hz}, \mathrm{C}\right), 129.1(\mathrm{CH}), 129.1(\mathrm{CH})$, $127.9(\mathrm{CH}), 126.5(\mathrm{CH}), 126.5(\mathrm{CH}), 83.0\left(\mathrm{~d}, J_{C-p}=155.2 \mathrm{~Hz}, \mathrm{CH}\right), 58.1(\mathrm{CH})$, $54.0\left(\mathrm{~d}, J_{C-P}=6.6 \mathrm{~Hz}, \mathrm{CH}_{3}\right), 53.5\left(\mathrm{~d}, J_{C-P}=6.7 \mathrm{~Hz} \mathrm{CH}\right.$ ), $43.7(\mathrm{C}), 42.1\left(\mathrm{CH}_{2}\right)$, $42.1\left(\mathrm{CH}_{2}\right), 42.1\left(\mathrm{CH}_{2}\right), 36.2\left(\mathrm{CH}_{2}\right), 36.2\left(\mathrm{CH}_{2}\right), 36.2\left(\mathrm{CH}_{2}\right), 29.5(\mathrm{CH}), 29.5(\mathrm{CH}), 29.5(\mathrm{CH}) .{ }^{1} \mathrm{H}$ NMR $\left(400 \mathrm{MHz}, \delta \mathrm{ppm} / \mathrm{CDCl}_{3}\right): 7.34-7.20(\mathrm{~m}, 5 \mathrm{H}), 4.88(\mathrm{dd}, J=28.4,9.6 \mathrm{~Hz}, 1 \mathrm{H}), 4.47(\mathrm{dd}, J=$ 17.6, $9.6 \mathrm{~Hz}, 1 \mathrm{H}), 3.85(\mathrm{~d}, J=10.8 \mathrm{~Hz}, 3 \mathrm{H}), 3.80(\mathrm{~d}, J=10.8 \mathrm{~Hz}, 3 \mathrm{H}), 2.04-2.00(\mathrm{~m}, 6 \mathrm{H}), 1.80-$ $1.58(\mathrm{~m}, 9 \mathrm{H}) .{ }^{31} \mathrm{P}$ NMR $\left(161.9 \mathrm{MHz}, \mathrm{CDCl}_{3}\right):$ 20.69. HRMS for $\mathrm{C}_{20} \mathrm{H}_{28} \mathrm{~N}_{3} \mathrm{NaO}_{3} \mathrm{P}^{+}$: calcd. [M+Na] $]^{+}$: 412.1760, found: 412.1760. FTIR ( $\left.\mathrm{cm}^{-1}\right): 3393,3020,2920,2400,1633,1403,1216,1050$, 929, 771, 669.

Compound 4ae: Dimethyl (1-(3-hydroxypropyl)-5-phenyl-4,5-dihydro-1H-1,2,3-triazol-4yl)phosphonate

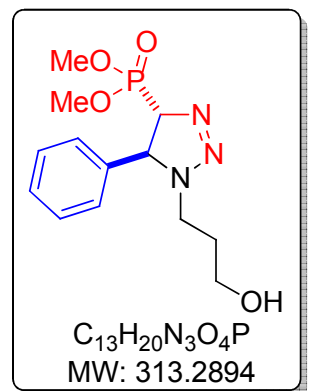

Following the general procedure, treatment of benzaldehyde $1 \mathbf{1 a}(50 \mathrm{mg}$, $0.47 \mathrm{mmol}$ ) with 3-amino-1-propanol $3 \mathrm{~m}(53 \mathrm{mg}, 0.71 \mathrm{mmol})$ and BOR (136 $\mathrm{mg}, 0.71 \mathrm{mmol}$ ) in $\mathrm{MeOH}(3 \mathrm{ml})$ at room temperature for $6 \mathrm{~h}$ followed by column chromatography afforded the product 4ae as a yellow solid ( $88 \mathrm{mg}, 60 \%$ ). $\mathbf{R}_{\mathbf{f}}$ (acetone/dichloromethane : 80/20) $=0.15$. $\mathrm{Mp}=138-140{ }^{\circ} \mathrm{C} .{ }^{13} \mathrm{C}$ NMR $\left(100 \mathrm{MHz}, \delta \mathrm{ppm} / \mathrm{CDCl}_{3}\right): 138.7\left(\mathrm{~d}, \mathrm{~J}_{\mathrm{C}-\mathrm{p}}=12.7\right.$ $\mathrm{Hz}, \mathrm{C}), 129.3(\mathrm{CH}), 129.3(\mathrm{CH}), 128.8(\mathrm{CH}), 127.0(\mathrm{CH}), 127.0(\mathrm{CH}), 81.7$ $\left(\mathrm{d}, J_{C-P}=158.5 \mathrm{~Hz}, \mathrm{CH}\right), 62.7(\mathrm{CH}), 59.6\left(\mathrm{CH}_{2}\right), 54.2\left(\mathrm{~d}, J_{C-P}=6.6 \mathrm{~Hz}, \mathrm{CH}_{3}\right), 53.4\left(\mathrm{~d}, J_{C-P}=6.8 \mathrm{~Hz}\right.$, $\left.\mathrm{CH}_{3}\right), 47.9\left(\mathrm{CH}_{2}\right), 30.7\left(\mathrm{CH}_{2}\right) .{ }^{1} \mathrm{H}$ NMR $\left(400 \mathrm{MHz}, \delta \mathrm{ppm} / \mathrm{CDCl}_{3}\right)$ : 7.37-7.30 (m, 3H), 7.21-7.19 $(\mathrm{m}, 2 \mathrm{H}), 4.75(\mathrm{dd}, J=26.4,10.4 \mathrm{~Hz}, 1 \mathrm{H}), 4.58(\mathrm{dd}, J=17.2,10.4 \mathrm{~Hz}, 1 \mathrm{H}), 3.85(\mathrm{~d}, J=10.8 \mathrm{~Hz}$, $3 \mathrm{H}), 3.83-3.77(\mathrm{~m}, 1 \mathrm{H}), 3.76(\mathrm{~d}, J=10.8 \mathrm{~Hz}, 3 \mathrm{H}), 3.67-3.62(\mathrm{~m}, 2 \mathrm{H}), 3.43-3.36(\mathrm{~m}, 1 \mathrm{H}), 2.63$ (OH), 1.88-1.82 (m, 2H). ${ }^{31} \mathrm{P}$ NMR (161.9 MHz, CDCl 3 ): 20.93. HRMS for $\mathrm{C}_{13} \mathrm{H}_{20} \mathrm{~N}_{3} \mathrm{NaO}_{4} \mathrm{P}^{+}$: calcd. $[\mathrm{M}+\mathrm{Na}]^{+}$: 336.1084 , found: 336.1082. FTIR $\left(\mathrm{cm}^{-1}\right): 3681,3397,3021,2402,2107$, $1627,1521,1414,1216,1050,928,761,670,564,496$.

Compound 4af: Dimethyl (1-(2-(diethylamino)ethyl)-5-phenyl-4,5-dihydro-1H-1,2,3-triazol4-yl)phosphonate

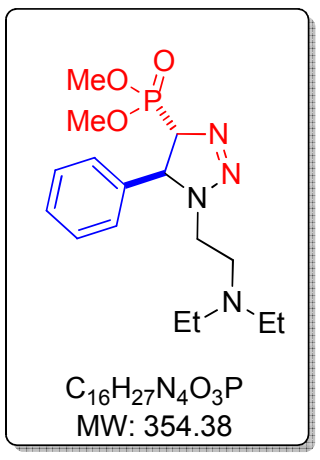

Following the general procedure, treatment of benzaldehyde 1a (50 $\mathrm{mg}, 0.47 \mathrm{mmol}$ ) with $\mathrm{N}, \mathrm{N}$, diethylethylenediamine $3 \mathrm{n}(82 \mathrm{mg}, 0.71$ $\mathrm{mmol}$ ) and $\mathrm{BOR}(136 \mathrm{mg}, 0.71 \mathrm{mmol})$ in $\mathrm{MeOH}(3 \mathrm{ml})$ at room temperature for $6 \mathrm{~h}$ followed by column chromatography afforded the 
product 4af as a yellow solid (129 $\mathrm{mg}, 77 \%$ ). $\mathbf{R}_{\mathbf{f}}$ (acetone/dichloromethane : 40/60) $=0.38$. $\mathrm{Mp}=145-147^{\circ} \mathrm{C} .{ }^{13} \mathrm{C} \mathbf{N M R}\left(100 \mathrm{MHz}, \delta \mathrm{ppm} / \mathrm{CDCl}_{3}\right): 138.9(\mathrm{C}), 129.3(\mathrm{CH}), 129.3(\mathrm{CH}), 128.8$ $(\mathrm{CH}), 127.3(\mathrm{CH}), 127.3(\mathrm{CH}), 82.0\left(\mathrm{~d}, J_{C-p}=159.6 \mathrm{~Hz}, \mathrm{CH}\right), 63.3(\mathrm{CH}), 54.0\left(\mathrm{~d}, J_{C-p}=6.9 \mathrm{~Hz}\right.$, $\left.\mathrm{CH}_{3}\right), 53.5\left(\mathrm{~d}, J_{C-P}=6.9 \mathrm{~Hz}, \mathrm{CH}_{3}\right), 51.2\left(\mathrm{CH}_{2}\right), 47.1\left(\mathrm{CH}_{2}\right), 47.1\left(\mathrm{CH}_{2}\right), 45.9\left(\mathrm{CH}_{2}\right), 11.6\left(\mathrm{CH}_{3}\right)$, $11.6\left(\mathrm{CH}_{3}\right) .{ }^{1} \mathbf{H}$ NMR $\left(400 \mathrm{MHz}, \delta \mathrm{ppm} / \mathrm{CDCl}_{3}\right):$ 7.38-7.32 (m, 3H), 7.24-7.22 (m, 2H), 4.85 (dd, $J=26.4,11.6 \mathrm{~Hz}, 1 \mathrm{H}), 4.56(\mathrm{dd}, J=18.0,11.6 \mathrm{~Hz}, 1 \mathrm{H}), 3.86-3.78(\mathrm{~m}, 1 \mathrm{H}), 3.85(\mathrm{~d}, J=10.8 \mathrm{~Hz}$, $3 \mathrm{H}), 3.79(\mathrm{~d}, J=10.8 \mathrm{~Hz}, 3 \mathrm{H}), 3.41-3.34(\mathrm{~m}, 1 \mathrm{H}), 3.76-2.70(\mathrm{~m}, 1 \mathrm{H}), 2.65-2.60(\mathrm{~m}, 1 \mathrm{H}), 2.48$ $(q, J=7.2,4 \mathrm{H}), 0.96(\mathrm{t}, J=7.2 \mathrm{~Hz}, 6 \mathrm{H}) .{ }^{31} \mathrm{P}$ NMR (161.9 MHz, $\left.\mathrm{CDCl}_{3}\right): 20.79$. HRMS for $\mathrm{C}_{16} \mathrm{H}_{28} \mathrm{~N}_{2} \mathrm{O}_{3} \mathrm{P}^{+}$: calcd. [M+H-N $]^{+}:$327.1832, found: 327.1831. FTIR $\left(\mathrm{cm}^{-1}\right)$ : 3392, 3019, 2400, $1647,1523,1404,1216,1049,929,758,669,627$.

Compound 4ag: Dimethyl (1-(3-morpholinopropyl)-5-phenyl-4,5-dihydro-1H-1,2,3-triazol4-yl)phosphonate

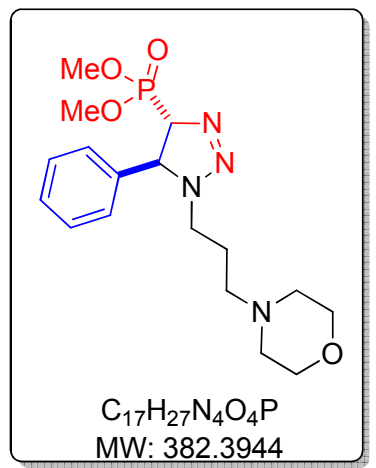

Following the general procedure, treatment of benzaldehyde $1 \mathbf{a}$ (50 $\mathrm{mg}, 0.47 \mathrm{mmol}$ ) with 4-(3-aminopropyl) morpholine 30 (52 $\mathrm{mg}, 0.71$ $\mathrm{mmol}$ ) and $\mathrm{BOR}(102 \mathrm{mg}, 0.71 \mathrm{mmol})$ in $\mathrm{MeOH}(3 \mathrm{ml})$ at room temperature for $6 \mathrm{~h}$ followed by column chromatography afforded the product $4 \mathrm{ag}$ as a white solid (126 $\mathrm{mg}, 70 \%) . \quad \mathbf{R}_{\mathbf{f}}$ (acetone/dichloromethane : 40/60) $=0.31 . \mathrm{Mp}=136-138{ }^{\circ} \mathrm{C} .{ }^{13} \mathrm{C}$ NMR $\left(100 \mathrm{MHz}, \delta \mathrm{ppm} / \mathrm{CDCl}_{3}\right): 138.6\left(\mathrm{~d}, J_{C-P}=12.7 \mathrm{~Hz}, \mathrm{C}\right), 129.3$ $(\mathrm{CH}), 129.3(\mathrm{CH}), 128.8(\mathrm{CH}), 127.2(\mathrm{CH}), 127.2(\mathrm{CH}), 82.0\left(\mathrm{~d}, J_{\mathrm{C}-p}=\right.$ $159.1 \mathrm{~Hz}, \mathrm{CH}), 67.0\left(\mathrm{CH}_{2}\right), 67.0\left(\mathrm{CH}_{2}\right), 63.0(\mathrm{CH}), 55.7\left(\mathrm{CH}_{2}\right), 54.1(\mathrm{~d}$, $\left.J_{C-P}=6.6 \mathrm{~Hz}, \mathrm{CH}_{3}\right), 53.7\left(\mathrm{CH}_{2}\right), 53.7\left(\mathrm{CH}_{2}\right), 53.4\left(\mathrm{~d}, \mathrm{~J}_{\mathrm{C}-\mathrm{P}}=6.8 \mathrm{~Hz}, \mathrm{CH}_{3}\right), 46.1\left(\mathrm{CH}_{2}\right), 25.0\left(\mathrm{CH}_{2}\right) .{ }^{1} \mathrm{H}$ NMR $\left(400 \mathrm{MHz}, \delta \mathrm{ppm} / \mathrm{CDCl}_{3}\right): 7.37-7.31(\mathrm{~m}, 3 \mathrm{H}), 7.23-7.21(\mathrm{~m}, 2 \mathrm{H}), 4.70(\mathrm{dd}, J=26.0,11.2$ $\mathrm{Hz}, 1 \mathrm{H}), 4.56(\mathrm{dd}, J=17.2,10.8 \mathrm{~Hz}, 1 \mathrm{H}), 3.85(\mathrm{~d}, J=10.4 \mathrm{~Hz}, 3 \mathrm{H}), 3.77(\mathrm{~d}, J=10.8 \mathrm{~Hz}, 3 \mathrm{H})$, 3.71-3.68 $(\mathrm{m}, 1 \mathrm{H}), 3.64(\mathrm{t}, J=4.8,4 \mathrm{H}), 3.39-3.33(\mathrm{~m}, 1 \mathrm{H}), 2.40-2.27(\mathrm{~m}, 6 \mathrm{H}), 1.84-1.76(\mathrm{~m}$, 2H). ${ }^{31} \mathrm{P}$ NMR (161.9 MHz, $\left.\mathrm{CDCl}_{3}\right)$ : 20.90. HRMS for $\mathrm{C}_{17} \mathrm{H}_{27} \mathrm{~N}_{4} \mathrm{NaO}_{4} \mathrm{P}^{+}$: calcd. [M+Na] $]^{+}$: 405.1662, found: 405.1661. FTIR ( $\left.\mathrm{cm}^{-1}\right)$ : 3391, 3020, 2400, 1651, 1403, 1216, 1049, 929,768, 669. 


\section{Optimization details for synthesis of 1,4,5-trisubstituted 1,2,3-triazoles}

Table S4: Optimization varying stoichiometry of $\mathrm{K}_{2} \mathrm{CO}_{3}$

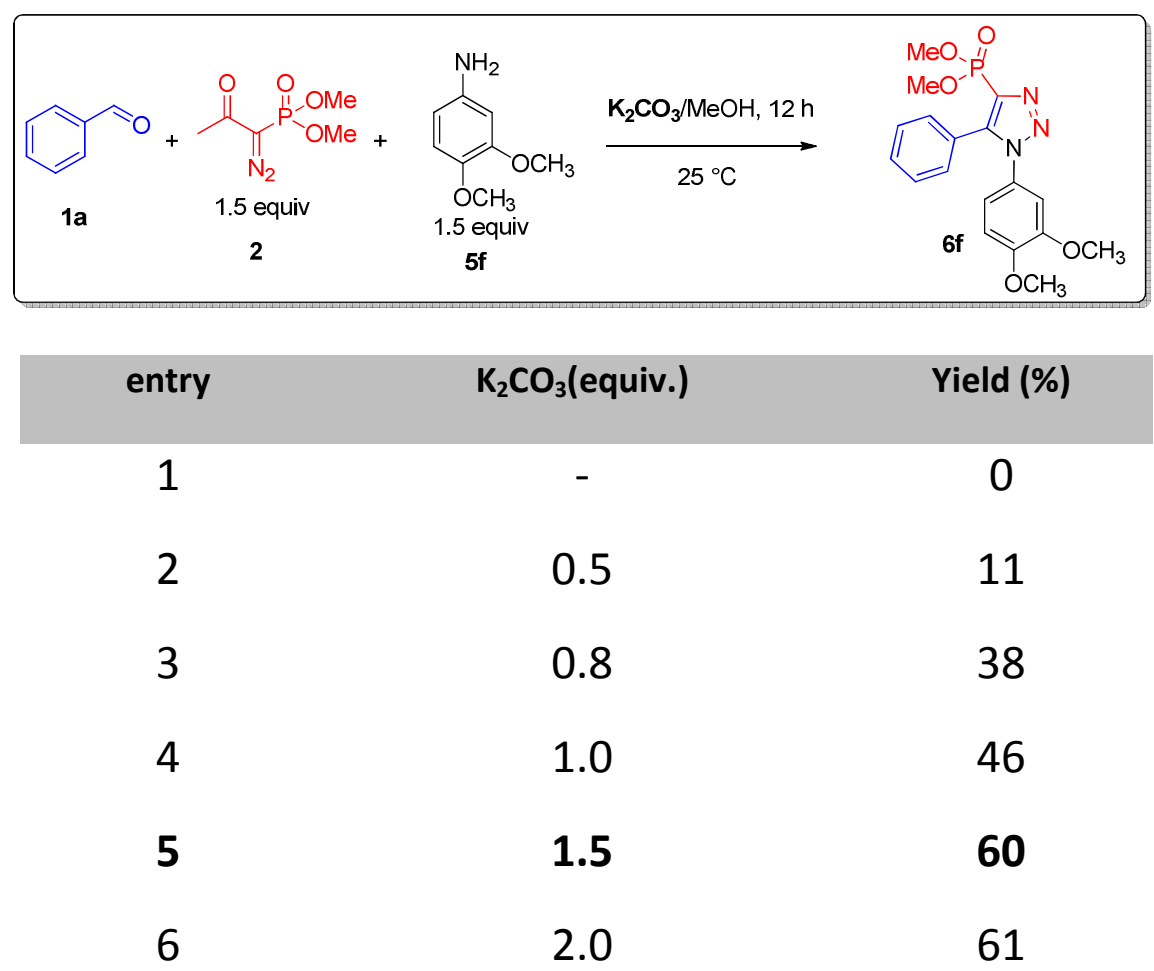

General procedure for the synthesis of phosphonyltriazoles 6

To an oven-dried round bottom flask was added benzaldehyde $1 \mathrm{a}(50 \mathrm{mg}, 0.47 \mathrm{mmol}$ ), aniline $5 \mathrm{a}(66 \mathrm{mg}, 0.71 \mathrm{mmol}), \mathrm{K}_{2} \mathrm{CO}_{3}(98 \mathrm{mg}, 0.71 \mathrm{mmol}$ ) and Bestmann-Ohira reagent 2 (136 mg, $0.71 \mathrm{mmol}$ ) and dissolved in $3 \mathrm{ml}$ of $\mathrm{MeOH}$. The reaction mixture was stirred at 25 ${ }^{\circ} \mathrm{C}$ for the specified time. After the completion of reaction, as indicated by TLC, solvent was evaporated off and extracted using ethyl acetate. The organic layer was dried over $\mathrm{Na}_{2} \mathrm{SO}_{4}$ and evaporated under reduced pressure. The residue was purified using column chromatography (100-200 mesh silica gel) using ethylacetate /hexane or Acetone/Dichloromethane as the eluent to afford the product 4.

\section{Characterization data of triazoline 6a-6I}

\section{Compound 6a: Dimethyl (1,5-diphenyl-1H-1,2,3-triazol-4-yl)phosphonate}

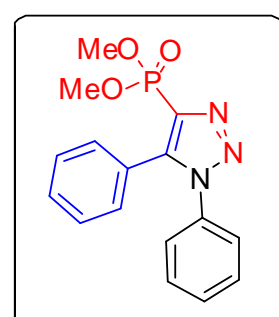

$\mathrm{C}_{16} \mathrm{H}_{16} \mathrm{~N}_{3} \mathrm{O}_{3} \mathrm{P}$ $\mathrm{MW}: 329.2903$
Following the general procedure, treatment of benzaldehyde 1 a $(50 \mathrm{mg}$, $0.47 \mathrm{mmol}$ ) with aniline $5 \mathrm{a}(66 \mathrm{mg}, 0.71 \mathrm{mmol})$ and BOR (136 mg, 0.71 $\mathrm{mmol})$ in the presence of $\mathrm{K}_{2} \mathrm{CO}_{3}(98 \mathrm{mg}, 0.71 \mathrm{mmol})$ in $\mathrm{MeOH}(3 \mathrm{ml})$ at room temperature for $12 \mathrm{~h}$ followed by column chromatography afforded the product $6 \mathbf{a}$ as a white solid (70 $\mathrm{mg}, 45 \%)$. $\mathbf{R}_{\mathbf{f}}$ (Ethyl acetate/Hexane : $50 / 50)=0.40 . \mathrm{Mp}=100-102{ }^{\circ} \mathrm{C} .{ }^{13} \mathrm{C}$ NMR $\left(100 \mathrm{MHz}, \delta \mathrm{ppm} / \mathrm{CDCl}_{3}\right): 143.2$ $\left(\mathrm{d}, J_{C-P}=33.2 \mathrm{~Hz}, \mathrm{C}\right), 136.4$ (C), 135.2 (d, $\left.J_{C-P}=239.4 \mathrm{~Hz}, \mathrm{C}\right), 130.2$ (C), 
$130.2(\mathrm{CH}), 130.2(\mathrm{CH}), 130.2(\mathrm{CH}), 129.7(\mathrm{CH}), 129.5(\mathrm{CH}), 129.5(\mathrm{CH}), 128.7(\mathrm{CH}), 128.7$ $(\mathrm{CH}), 125.4(\mathrm{CH}), 125.4(\mathrm{CH}), 53.6\left(\mathrm{~d}, J_{C-P}=5.8 \mathrm{~Hz}, \mathrm{CH}_{3}\right), 53.6\left(\mathrm{~d}, J_{C-P}=5.8 \mathrm{~Hz}, \mathrm{CH}_{3}\right) .{ }^{1} \mathrm{H} \mathbf{N M R}$ $\left(400 \mathrm{MHz}, \delta \mathrm{ppm} / \mathrm{CDCl}_{3}\right): 7.32-7.18(\mathrm{~m}, 10 \mathrm{H}), 3.72(\mathrm{~d}, J=11.2 \mathrm{~Hz}, 6 \mathrm{H}) .{ }^{31} \mathbf{P}$ NMR $(161.9 \mathrm{MHz}$, $\mathrm{CDCl}_{3}$ ): 9.77. HRMS for $\mathrm{C}_{16} \mathrm{H}_{17} \mathrm{~N}_{3} \mathrm{O}_{3} \mathrm{P}^{+}$: calcd. $[\mathrm{M}+\mathrm{H}]^{+}: 330.1002$, found: 330.0997 . FTIR $\left(\mathrm{cm}^{-1}\right)$ : $3390,2925,1642,1402,1047,759$.

Compound 6b: Dimethyl (1-(4-methoxyphenyl)-5-phenyl-1H-1,2,3-triazol-4yl)phosphonate

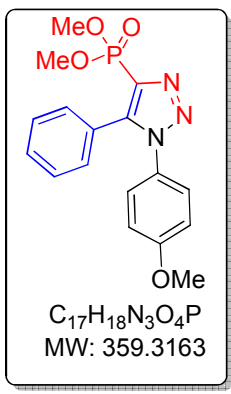

Following the general procedure, treatment of benzaldehyde 1a $(50 \mathrm{mg}$, $0.47 \mathrm{mmol}$ ) with 4-methoxyaniline $5 \mathrm{~b}(87 \mathrm{mg}, 0.71 \mathrm{mmol})$ and BOR (136 mg, $0.71 \mathrm{mmol})$ in the presence of $\mathrm{K}_{2} \mathrm{CO}_{3}(98 \mathrm{mg}, 0.71 \mathrm{mmol})$ in $\mathrm{MeOH}(3 \mathrm{ml})$ at room temperature for $12 \mathrm{~h}$ followed by column chromatography afforded the product $\mathbf{6 b}$ as a white solid $(79 \mathrm{mg}, 47 \%)$. $\mathbf{R}_{\mathbf{f}}$ (Ethyl acetate/Hexane : $50 / 50)=0.22 . \mathrm{Mp}=110-112{ }^{\circ} \mathrm{C} .{ }^{13} \mathrm{C}$ NMR $\left(100 \mathrm{MHz}, \delta \mathrm{ppm} / \mathrm{CDCl}_{3}\right): 160.4$ (C), $143.1\left(\mathrm{~d}, J_{C-P}=33.2 \mathrm{~Hz}, \mathrm{C}\right), 134.9\left(\mathrm{~d}, J_{C-P}=239.2 \mathrm{~Hz}, \mathrm{C}\right), 130.2(\mathrm{CH}), 130.2$ $(\mathrm{CH}), 130.1(\mathrm{CH}), 128.8(\mathrm{C}), 128.7(\mathrm{CH}), 128.7(\mathrm{CH}), 126.8(\mathrm{CH}), 126.8(\mathrm{CH}), 125.6(\mathrm{C}), 114.2$ $(\mathrm{CH}), 114.2(\mathrm{CH}), 55.7\left(\mathrm{CH}_{3}\right), 53.6\left(\mathrm{~d}, J_{C-P}=5.8 \mathrm{~Hz}, \mathrm{CH}_{3}\right), 53.6\left(\mathrm{~d}, J_{C-P}=5.8 \mathrm{~Hz}, \mathrm{CH}_{3}\right) .{ }^{1} \mathrm{H} \mathbf{N M R}$ $\left(400 \mathrm{MHz}, \delta \mathrm{ppm} / \mathrm{CDCl}_{3}\right): 7.41-7.35(\mathrm{~m}, 5 \mathrm{H}), 7.19(\mathrm{~d}, J=8.8 \mathrm{~Hz}, 2 \mathrm{H}), 6.87(\mathrm{~d}, J=8.8 \mathrm{~Hz}, 2 \mathrm{H})$, $3.81(\mathrm{~s}, 3 \mathrm{H}), 3.80(\mathrm{~d}, J=10.4 \mathrm{~Hz}, 6 \mathrm{H}) .{ }^{31} \mathrm{P}$ NMR (161.9 MHz, CDCl 3 ): 9.93. HRMS for $\mathrm{C}_{17} \mathrm{H}_{19} \mathrm{~N}_{3} \mathrm{O}_{4} \mathrm{P}^{+}$: calcd. $[\mathrm{M}+\mathrm{H}]^{+}: 360.1108$, found: 360.1103. FTIR $\left(\mathrm{cm}^{-1}\right): 3392,3020,2400$, $1646,1515,1404,1216,1047,928,835,758,669,627,576$.

\section{Compound 6c: Dimethyl (1-(4-bromophenyl)-5-phenyl-1H-1,2,3-triazol-4-yl)phosphonate}

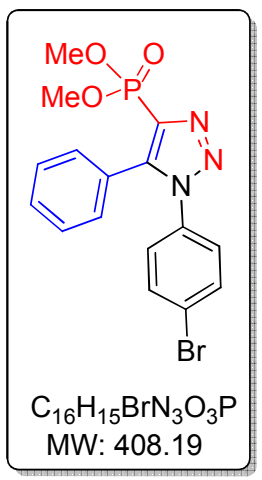

Following the general procedure, treatment of benzaldehyde $1 \mathrm{a}(50 \mathrm{mg}$, $0.47 \mathrm{mmol}$ ) with 4-bromoaniline $5 \mathrm{c}(122 \mathrm{mg}, 0.71 \mathrm{mmol})$ and BOR (136 mg, $0.71 \mathrm{mmol}$ ) in the presence of $\mathrm{K}_{2} \mathrm{CO}_{3}(98 \mathrm{mg}, 0.71 \mathrm{mmol})$ in $\mathrm{MeOH}(3 \mathrm{ml})$ at room temperature for $12 \mathrm{~h}$ followed by column chromatography afforded the product $6 \mathrm{c}$ as a white solid (100 $\mathrm{mg}, 52 \%)$. $\mathbf{R}_{\mathbf{f}}$ (Ethyl acetate/Hexane : $50 / 50)=0.46 . \mathrm{Mp}=148-150{ }^{\circ} \mathrm{C} .{ }^{13} \mathrm{C} \mathrm{NMR}\left(100 \mathrm{MHz}, \delta \mathrm{ppm} / \mathrm{CDCl}_{3}\right): 143.1$ $\left(d, J_{C-p}=26.9 \mathrm{~Hz}, \mathrm{C}\right), 135.4\left(\mathrm{~d}, J_{C-P}=191.3 \mathrm{~Hz}, \mathrm{C}\right), 134.8(\mathrm{C}), 132.7(\mathrm{CH}), 132.7$ $(\mathrm{CH}), 130.4(\mathrm{CH}), 130.1(\mathrm{CH}), 130.1(\mathrm{CH}), 128.9(\mathrm{CH}), 128.9(\mathrm{CH}), 126.7(\mathrm{CH})$, $126.7(\mathrm{CH}), 124.4\left(\mathrm{~d}, \mathrm{~J}_{\mathrm{C}-\mathrm{p}}=130.1 \mathrm{~Hz}, \mathrm{C}\right), 115.6(\mathrm{C}), 53.7\left(\mathrm{~d}, \mathrm{~J}_{\mathrm{C}-\mathrm{p}}=4.6 \mathrm{~Hz}, \mathrm{CH}_{3}\right)$, $53.7\left(\mathrm{~d}, J_{C-P}=4.6 \mathrm{~Hz}, \mathrm{CH}_{3}\right) .{ }^{1} \mathrm{H}$ NMR $\left(400 \mathrm{MHz}, \delta \mathrm{ppm} / \mathrm{CDCl}_{3}\right): 7.52(\mathrm{dd}, J=8.0,1.6 \mathrm{~Hz}, 2 \mathrm{H})$, 7.43-7.37 (m, 3H), 7.33-7.32 (m, 2H), 7.15 (dd, J=5.6, 1.6 Hz, 2H), 3.79 (d, J=9.2 Hz, 6H). ${ }^{31} \mathbf{P}$ NMR (161.9 MHz, $\mathrm{CDCl}_{3}$ ): 9.38. HRMS for $\mathrm{C}_{16} \mathrm{H}_{16} \mathrm{BrN}_{3} \mathrm{O}_{3} \mathrm{P}^{+}$: calcd. [M+H] $]^{+}: 408.0107$, found: 408.0104. FTIR (cm $\left.{ }^{-1}\right): 3392,3019,2957,2854,2400,1645,1493,1402,1041,995,928,669$, $633,576$. 


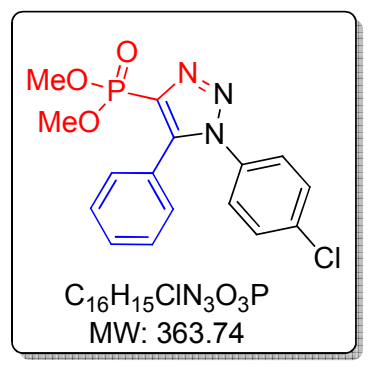

Following the general procedure, treatment of benzaldehyde 1a (50 $\mathrm{mg}, 0.47 \mathrm{mmol}$ ) with 4-chloroaniline $5 \mathbf{d}(90 \mathrm{mg}, 0.71 \mathrm{mmol}$ ) and BOR (136 mg, $0.71 \mathrm{mmol}$ ) in the presence of $\mathrm{K}_{2} \mathrm{CO}_{3}(98 \mathrm{mg}, 0.71 \mathrm{mmol}$ ) in $\mathrm{MeOH}(3 \mathrm{ml})$ at room temperature for $12 \mathrm{~h}$ followed by column chromatography afforded the product $\mathbf{6 d}$ as a white solid $(88 \mathrm{mg}$, 51\%). $\mathbf{R}_{\mathbf{f}}$ (Ethyl acetate/Hexane : 50/50) $=0.38 . \mathbf{M p}=140-142{ }^{\circ} \mathrm{C} .{ }^{13} \mathrm{C}$

NMR $\left(100 \mathrm{MHz}, \delta \mathrm{ppm} / \mathrm{CDCl}_{3}\right): 143.1\left(\mathrm{~d}, J_{C-P}=27.6 \mathrm{~Hz}, \mathrm{C}\right), 135.4(\mathrm{~d}$, $\left.J_{C-P}=191.2 \mathrm{~Hz}, \mathrm{C}\right), 135.0\left(\mathrm{~d}, J_{C-P}=142.1 \mathrm{~Hz}, \mathrm{C}\right), 130.4(\mathrm{CH}), 130.1(\mathrm{CH}), 130.1(\mathrm{CH}), 129.8$ $(\mathrm{CH}), 129.8(\mathrm{CH}), 128.9(\mathrm{CH}), 128.9(\mathrm{CH}), 126.5(\mathrm{CH}), 126.5(\mathrm{CH}), 125.1(\mathrm{C}), 115.1(\mathrm{C}), 53.7(\mathrm{~d}$, $\left.J_{C-P}=4.6 \mathrm{~Hz}, \mathrm{CH}_{3}\right), 53.7\left(\mathrm{~d}, J_{C-P}=4.6 \mathrm{~Hz}, \mathrm{CH}_{3}\right) .{ }^{1} \mathrm{H}$ NMR $\left(400 \mathrm{MHz}, \delta \mathrm{ppm} / \mathrm{CDCl}_{3}\right):$ 7.44-7.32 (m, 7H), $7.22(\mathrm{~d}, J=7.2 \mathrm{~Hz}, 2 \mathrm{H}), 3.79(\mathrm{~d}, J=9.2 \mathrm{~Hz}, 6 \mathrm{H}) .{ }^{31} \mathrm{P}$ NMR $\left(161.9 \mathrm{MHz}, \mathrm{CDCl}_{3}\right):$ 9.41. HRMS for $\mathrm{C}_{16} \mathrm{H}_{16} \mathrm{ClN}_{3} \mathrm{O}_{3} \mathrm{P}^{+}$: calcd. [M+H] $]^{+}: 364.0612$, found: 364.0650. FTIR $\left(\mathrm{cm}^{-1}\right): 3683,3019$, 2956, 2853, 2400, 1896, 1602, 1496, 1094, 1000, 928, 833, 669, 639, 577.

\section{Compound 6e: Dimethyl (1-(4-fluorophenyl)-5-phenyl-1H-1,2,3-triazol-4-yl)phosphonate}

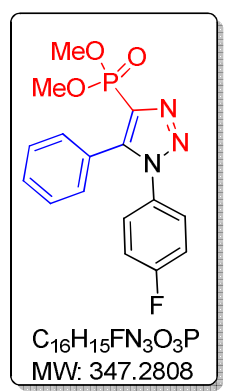

Following the general procedure, treatment of benzaldehyde $1 \mathrm{1a}(50 \mathrm{mg}, 0.47$ mmol) with 4-fluoroaniline 5e $(79 \mathrm{mg}, 0.71 \mathrm{mmol})$ and BOR (136 mg, 0.71 $\mathrm{mmol})$ in the presence of $\mathrm{K}_{2} \mathrm{CO}_{3}(98 \mathrm{mg}, 0.71 \mathrm{mmol})$ in $\mathrm{MeOH}(3 \mathrm{ml})$ at room temperature for $12 \mathrm{~h}$ followed by column chromatography afforded the product $\mathbf{6 e}$ as a white solid (103 $\mathrm{mg}, 63 \%) . \mathbf{R}_{\mathbf{f}}$ (Ethyl acetate/Hexane : 50/50) $=0.20 . \mathrm{Mp}=112-114{ }^{\circ} \mathrm{C} .{ }^{13} \mathrm{C}$ NMR $\left(100 \mathrm{MHz}, \delta \mathrm{ppm} / \mathrm{CDCl}_{3}\right): 162.9$ (d, $J_{C-F}=$ $249.3 \mathrm{~Hz}, \mathrm{C}), 143.2$ (d, $\left.J_{C-P}=33.3 \mathrm{~Hz}, \mathrm{C}\right), 135.3$ (d, $\left.J_{C-P}=239.3 \mathrm{~Hz}, \mathrm{C}\right), 132.0$

(C), $130.3(\mathrm{CH}), 130.1(\mathrm{CH}), 130.1(\mathrm{CH}), 128.8(\mathrm{CH}), 128.8(\mathrm{CH}), 127.3(\mathrm{CH}), 127.3(\mathrm{CH}), 125.2$ (C), $116.7(\mathrm{CH}), 116.5(\mathrm{CH}), 53.6\left(\mathrm{~d}, J_{C-P}=5.8 \mathrm{~Hz}, \mathrm{CH}_{3}\right), 53.6\left(\mathrm{~d}, J_{C-P}=5.8 \mathrm{~Hz}, \mathrm{CH}_{3}\right) .{ }^{1} \mathrm{H} \mathbf{N M R}$ $\left(400 \mathrm{MHz}, \delta \mathrm{ppm} / \mathrm{CDCl}_{3}\right): 7.33-7.32(\mathrm{~m}, 3 \mathrm{H}), 7.27(\mathrm{~d}, J=1.2 \mathrm{~Hz}, 2 \mathrm{H}), 7.21(\mathrm{q}, J=4.0 \mathrm{~Hz}, 2 \mathrm{H})$, 7.04-7.00 (m, 2H), $3.74(\mathrm{~d}, J=8.8 \mathrm{~Hz}, 6 \mathrm{H}) .{ }^{31} \mathrm{P}$ NMR $\left(161.9 \mathrm{MHz}, \mathrm{CDCl}_{3}\right)$ : 9.53. HRMS for $\mathrm{C}_{16} \mathrm{H}_{16} \mathrm{FN}_{3} \mathrm{O}_{3} \mathrm{P}^{+}$: calcd. [M+H] $]^{+}: 348.0908$, found: 348.0913. FTIR $\left(\mathrm{cm}^{-1}\right): 3854,3686,3019$, 2928, 2400, 1603, 1514, 1476, 1420, 1216, 1037, 929, 758, 669, 627.

\section{Compound 6f: Dimethyl (1-(3,4-dimethoxyphenyl)-5-phenyl-1H-1,2,3-triazol-4- yl)phosphonate}

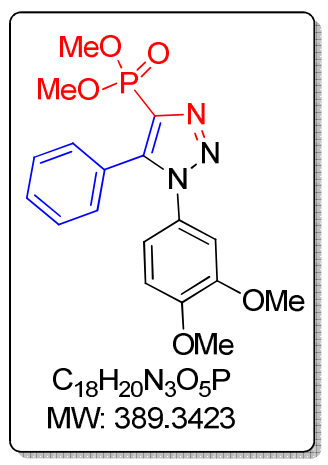

Following the general procedure, treatment of benzaldehyde 1a (50 $\mathrm{mg}, 0.47 \mathrm{mmol}$ ) with 3,4-dimethoxyaniline $5 \mathbf{f}(108 \mathrm{mg}, 0.71 \mathrm{mmol})$ and BOR (136 mg, $0.71 \mathrm{mmol})$ in the presence of $\mathrm{K}_{2} \mathrm{CO}_{3}(98 \mathrm{mg}, 0.71 \mathrm{mmol})$ in $\mathrm{MeOH}(3 \mathrm{ml})$ at room temperature for $12 \mathrm{~h}$ followed by column chromatography afforded the product $6 \mathbf{f}$ as a white solid $(110 \mathrm{mg}$, 60\%). $\mathbf{R}_{\mathbf{f}}$ (Ethyl acetate/Hexane : 50/50) $=0.18 . \mathbf{M p}=136-138{ }^{\circ} \mathrm{C} .{ }^{13} \mathbf{C}$ NMR (100 MHz, $\delta \mathrm{ppm} / \mathrm{CDCl}_{3}$ ): 149.8 (C), 149.2 (C), 142.5 (d, J $J_{C-p}=26.6$ $\mathrm{Hz}, \mathrm{C}), 134.9\left(\mathrm{~d}, J_{C-P}=191.3 \mathrm{~Hz}, \mathrm{C}\right), 130.1(\mathrm{C}), 130.1(\mathrm{CH}), 130.1(\mathrm{CH})$, 
$128.7(\mathrm{CH}), 128.6(\mathrm{CH}), 128.6(\mathrm{CH}), 125.6(\mathrm{C}) 117.7(\mathrm{CH}), 110.9(\mathrm{CH}), 108.8(\mathrm{CH}), 56.1\left(\mathrm{CH}_{3}\right)$, $56.0\left(\mathrm{CH}_{3}\right), 53.5\left(\mathrm{~d}, \mathrm{~J}_{\mathrm{C}-\mathrm{P}}=4.5 \mathrm{~Hz}, \mathrm{CH}_{3}\right), 53.5\left(\mathrm{~d}, \mathrm{~J}_{\mathrm{C}-\mathrm{P}}=4.5 \mathrm{~Hz}, \mathrm{CH}_{3}\right) .{ }^{1} \mathbf{H}$ NMR $(400 \mathrm{MHz}, \delta$ $\left.\mathrm{ppm} / \mathrm{CDCl}_{3}\right)$ : 7.37-7.34 (m, 5H), $6.80(\mathrm{~s}, 2 \mathrm{H}), 6.74(\mathrm{~s}, 1 \mathrm{H}), 3.85(\mathrm{~s}, 3 \mathrm{H}), 3.77(\mathrm{~d}, J=11.2 \mathrm{~Hz}$, $6 \mathrm{H}), 3.67$ (s, 3H). ${ }^{31} \mathrm{P}$ NMR (161.9 MHz, $\mathrm{CDCl}_{3}$ ): 9.80. HRMS for $\mathrm{C}_{18} \mathrm{H}_{21} \mathrm{~N}_{3} \mathrm{O}_{5} \mathrm{P}^{+}$: calcd. $[\mathrm{M}+\mathrm{H}]^{+}$: 390.1213, found: 390.1213. FTIR ( $\left.\mathrm{cm}^{-1}\right): 3392,3019,2957,2853,2400,1602,1518,1465$, 1403, 1254, 1134, 1040, 928, 879, 669.

\section{Compound 6g: Dimethyl (1-(3-methoxyphenyl)-5-phenyl-1H-1,2,3-triazol-4- yl)phosphonate}

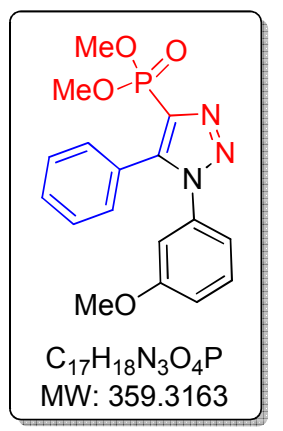

Following the general procedure, treatment of benzaldehyde 1a $(50 \mathrm{mg}$, $0.47 \mathrm{mmol}$ ) with 3-methoxyaniline $5 \mathrm{~g}(87 \mathrm{mg}, 0.71 \mathrm{mmol})$ and BOR (136 $\mathrm{mg}, 0.71 \mathrm{mmol})$ in the presence of $\mathrm{K}_{2} \mathrm{CO}_{3}(98 \mathrm{mg}, 0.71 \mathrm{mmol})$ in $\mathrm{MeOH}$ $(3 \mathrm{ml}$ ) at room temperature for $12 \mathrm{~h}$ followed by column chromatography afforded the product $\mathbf{6 g}$ as a white solid (90 mg, 53\%). $\mathbf{R}_{\mathbf{f}}$ (Ethyl acetate/Hexane : 50/50) $=0.20 . \mathrm{Mp}=138-140{ }^{\circ} \mathrm{C} .{ }^{13} \mathrm{C}$ NMR $(100 \mathrm{MHz}, \delta$ $\mathrm{ppm} / \mathrm{CDCl}_{3}$ ): 160.2 (C), 143.1 (d, $\left.J_{C-P}=26.6 \mathrm{~Hz}, \mathrm{C}\right), 136.7$ (C), 135.1 (d, $J_{C-P}=$ $191.1 \mathrm{~Hz}, \mathrm{C}), 130.2(\mathrm{CH}), 130.2(\mathrm{CH}), 130.1(\mathrm{CH}), 130.1(\mathrm{CH}), 128.7(\mathrm{CH})$, $128.7(\mathrm{CH}), 125.5(\mathrm{C}), 117.4(\mathrm{CH}), 115.8(\mathrm{CH}), 110.8(\mathrm{CH}), 55.5\left(\mathrm{CH}_{3}\right), 53.6\left(\mathrm{~d}, J_{C-p}=4.6 \mathrm{~Hz}\right.$, $\left.\mathrm{CH}_{3}\right), 53.6\left(\mathrm{~d}, \mathrm{~J}_{\mathrm{C}-\mathrm{P}}=4.6 \mathrm{~Hz}, \mathrm{CH}_{3}\right) .{ }^{1} \mathrm{H}$ NMR $\left(400 \mathrm{MHz}, \delta \mathrm{ppm} / \mathrm{CDCl}_{3}\right): 7.41-7.36(\mathrm{~m}, 5 \mathrm{H})$, 7.27$7.23(\mathrm{~m}, 1 \mathrm{H}), 6.93(\mathrm{~d}, J=8.4 \mathrm{~Hz}, 1 \mathrm{H}), 6.83-6.80(\mathrm{~m}, 2 \mathrm{H}), 3.80(\mathrm{~d}, J=11.2 \mathrm{~Hz}, 6 \mathrm{H}) 0.3 .69(\mathrm{~s}$, 3H). ${ }^{31} \mathrm{P}$ NMR (161.9 MHz, $\mathrm{CDCl}_{3}$ ): 9.71. HRMS for $\mathrm{C}_{17} \mathrm{H}_{19} \mathrm{~N}_{3} \mathrm{O}_{4} \mathrm{P}^{+}$: calcd. $[\mathrm{M}+\mathrm{H}]^{+}: 360.1108$, found: 360.1112 . FTIR $\left(\mathrm{cm}^{-1}\right)$ : 3682, 3020, 2400, 1609, 1523, 1497, 1474, 1405, 1331, 1216, $1043,928,758,699,627$.

\section{Compound 6h: Dimethyl (1-(3,4-dimethylphenyl)-5-phenyl-1H-1,2,3-triazol-4- yl)phosphonate}

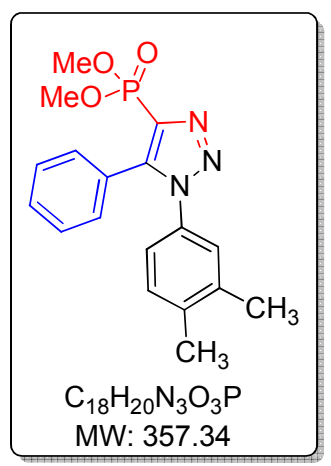

Following the general procedure, treatment of benzaldehyde 1a (50 $\mathrm{mg}, 0.47 \mathrm{mmol}$ ) with 3, 4-dimethylaniline $5 \mathrm{~h}(91 \mathrm{mg}, 0.71 \mathrm{mmol})$ and BOR (136 mg, $0.71 \mathrm{mmol}$ ) in the presence of $\mathrm{K}_{2} \mathrm{CO}_{3}(98 \mathrm{mg}, 0.71 \mathrm{mmol}$ ) in $\mathrm{MeOH}(3 \mathrm{ml})$ at room temperature for $12 \mathrm{~h}$ followed by column chromatography afforded the product $6 \mathrm{~h}$ as a white solid $(79 \mathrm{mg}$, 47\%). $\mathbf{R}_{\mathbf{f}}$ (Ethyl acetate/Hexane : 50/50) $=0.44 . \mathbf{M p}=135-137{ }^{\circ} \mathrm{C} .{ }^{13} \mathbf{C}$ NMR $\left(100 \mathrm{MHz}, \delta \mathrm{ppm} / \mathrm{CDCl}_{3}\right): 143.0\left(\mathrm{~d}, J_{C-P}=33.3 \mathrm{~Hz}, \mathrm{C}\right), 138.5$ (C), $138.2(\mathrm{C}), 134.9$ (d, $\left.J_{C-p}=239.2 \mathrm{~Hz}, \mathrm{C}\right), 133.6(\mathrm{C}), 130.3(\mathrm{CH}), 130.2(\mathrm{CH})$, $130.2(\mathrm{CH}), 130.0(\mathrm{CH}), 128.6(\mathrm{CH}), 128.6(\mathrm{CH}), 126.3(\mathrm{CH}), 125.7(\mathrm{C})$, $122.5(\mathrm{CH}), 53.6\left(\mathrm{~d}, J_{C-p}=5.9 \mathrm{~Hz}, \mathrm{CH}_{3}\right), 53.6\left(\mathrm{~d}, J_{\mathrm{C}-\mathrm{P}}=5.9 \mathrm{~Hz}, \mathrm{CH}_{3}\right), 19.8\left(\mathrm{CH}_{3}\right), 19.6\left(\mathrm{CH}_{3}\right) .{ }^{1} \mathrm{H}$ NMR $\left(400 \mathrm{MHz}, \delta \mathrm{ppm} / \mathrm{CDCl}_{3}\right): 7.40-7.35(\mathrm{~m}, 5 \mathrm{H}), 7.14(\mathrm{~d}, J=1.2 \mathrm{~Hz}, 1 \mathrm{H}), 7.08(\mathrm{~d}, J=6.8 \mathrm{~Hz}$, $1 \mathrm{H}), 6.87(\mathrm{dd}, J=6.4,1.6 \mathrm{~Hz}, 1 \mathrm{H}), 3.80(\mathrm{~d}, J=9.2 \mathrm{~Hz}, 6 \mathrm{H}), 2.26(\mathrm{~s}, 3 \mathrm{H}), 2.22(\mathrm{~s}, 3 \mathrm{H}) .{ }^{31} \mathrm{P} \mathrm{NMR}$ (161.9 MHz, $\mathrm{CDCl}_{3}$ ): 9.99. HRMS for $\mathrm{C}_{18} \mathrm{H}_{21} \mathrm{~N}_{3} \mathrm{O}_{3} \mathrm{P}^{+}$: calcd. $[\mathrm{M}+\mathrm{H}]^{+}$: 358.1315, found: 358.1319. FTIR (cm $\left.{ }^{-1}\right):$ 3391, 3019, 2927, 2398, 1651, 1517, 1403, 1216, 1043, 928, 770, 669. 


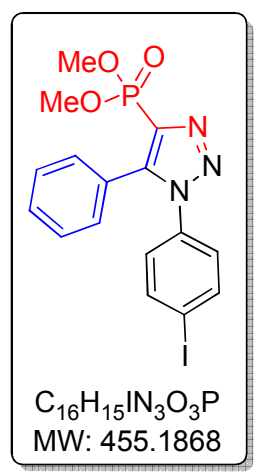

Following the general procedure, treatment of benzaldehyde $1 \mathrm{a}(50 \mathrm{mg}$, $0.47 \mathrm{mmol}$ ) with 4-iodoaniline $5 \mathbf{i}(155 \mathrm{mg}, 0.71 \mathrm{mmol})$ and BOR $(136 \mathrm{mg}$, $0.71 \mathrm{mmol})$ in the presence of $\mathrm{K}_{2} \mathrm{CO}_{3}(98 \mathrm{mg}, 0.71 \mathrm{mmol})$ in $\mathrm{MeOH}(3 \mathrm{ml})$ at room temperature for $12 \mathrm{~h}$ followed by column chromatography afforded the product $\mathbf{6 i}$ as a white solid (135 $\mathrm{mg}, 63 \%)$. $\mathbf{R}_{\mathbf{f}}$ (Ethyl acetate/Hexane : $50 / 50)=0.38 . \mathrm{Mp}=165-167{ }^{\circ} \mathrm{C} .{ }^{13} \mathrm{C}$ NMR $\left(100 \mathrm{MHz}, \delta \mathrm{ppm} / \mathrm{CDCl}_{3}\right): 143.0$ (d, $\left.J_{C-P}=27.4 \mathrm{~Hz}, \mathrm{C}\right), 138.7(\mathrm{CH}), 138.7(\mathrm{CH}), 135.5\left(\mathrm{~d}, J_{C-P}=190.9 \mathrm{~Hz}, \mathrm{C}\right)$, $135.5(\mathrm{C}), 130.4(\mathrm{CH}), 130.1(\mathrm{CH}), 130.1(\mathrm{CH}), 128.9(\mathrm{CH}), 128.9(\mathrm{CH}), 126.8$ $(\mathrm{CH}), 126.8(\mathrm{CH}), 125.1(\mathrm{C}), 95.3(\mathrm{C}), 53.6\left(\mathrm{~d}, \mathrm{~J}_{\mathrm{C}-\mathrm{P}}=4.5 \mathrm{~Hz}, \mathrm{CH}_{3}\right), 53.6\left(\mathrm{~d}, \mathrm{~J}_{\mathrm{C}-\mathrm{P}}=\right.$

$\left.4.5 \mathrm{~Hz}, \mathrm{CH}_{3}\right) .{ }^{1} \mathrm{H}$ NMR $\left(400 \mathrm{MHz}, \delta \mathrm{ppm} / \mathrm{CDCl}_{3}\right): 7.72(\mathrm{~d}, J=8.0 \mathrm{~Hz}, 2 \mathrm{H}), 7.41-7.34(\mathrm{~m}, 5 \mathrm{H})$, 7.03-7.01 (d, $J=7.6 \mathrm{~Hz}, 2 \mathrm{H}), 3.79(\mathrm{~d}, J=11.2 \mathrm{~Hz}, 6 \mathrm{H}) .{ }^{31} \mathrm{P}$ NMR $\left(161.9 \mathrm{MHz}, \mathrm{CDCl}_{3}\right): 9.35$. HRMS for $\mathrm{C}_{16} \mathrm{H}_{16} \mathrm{IN}_{3} \mathrm{O}_{3} \mathrm{P}^{+}$: calcd. [M+H] $]^{+}$455.9966, found: 455.9978. FTIR (cm $\left.{ }^{-1}\right): 3392,3020$, 2927, 2400, 1651, 1403, 1216, 1055, 929, 679, 669.

\section{Compound 6j: Dimethyl (1-(4-iodophenyl)-5-(4-methoxyphenyl)-1H-1,2,3-triazol-4- yl)phosphonate}

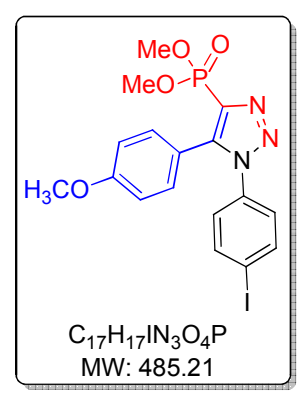

Following the general procedure, treatment of 4-methoxy benzaldehyde 1b $(50 \mathrm{mg}, 0.37 \mathrm{mmol}$ ) with 4-iodoaniline $5 \mathbf{i}(121 \mathrm{mg}, 0.55 \mathrm{mmol})$ and BOR (106 mg, $0.55 \mathrm{mmol}$ ) in the presence of $\mathrm{K}_{2} \mathrm{CO}_{3}(76 \mathrm{mg}, 0.55 \mathrm{mmol})$ in $\mathrm{MeOH}(3 \mathrm{ml})$ at room temperature for $12 \mathrm{~h}$ followed by column chromatography afforded the product $\mathbf{6} \mathbf{j}$ as a white solid (55 $\mathrm{mg}, 31 \%$ ). $\mathbf{R}_{\mathbf{f}}$ (Ethyl acetate/Hexane : 50/50) $=0.15 . \mathrm{Mp}=150-152{ }^{\circ} \mathrm{C} .{ }^{13} \mathrm{C}$ NMR $(100$ $\left.\mathrm{MHz}, \delta \mathrm{ppm} / \mathrm{CDCl}_{3}\right): 161.1(\mathrm{C}), 143.1\left(\mathrm{~d}, J_{C-P}=33.0 \mathrm{~Hz}, \mathrm{C}\right) 138.7(\mathrm{CH}), 138.7$

$(\mathrm{CH}), 135.7(\mathrm{C}), 131.6(\mathrm{CH}), 131.6(\mathrm{CH}), 129.5(\mathrm{C}), 126.9(\mathrm{CH}), 126.9(\mathrm{CH}), 116.9(\mathrm{C}), 114.4$ $(\mathrm{CH}), 114.4(\mathrm{CH}), 95.2(\mathrm{C}), 55.4\left(\mathrm{CH}_{3}\right), 53.7\left(\mathrm{~d}, \mathrm{~J}_{C-P}=6.1 \mathrm{~Hz}, \mathrm{CH}_{3}\right), 53.7\left(\mathrm{~d}, \mathrm{~J}_{C-P}=6.1 \mathrm{~Hz}, \mathrm{CH}_{3}\right) .{ }^{1} \mathrm{H}$ NMR $\left(400 \mathrm{MHz}, \delta \mathrm{ppm} / \mathrm{CDCl}_{3}\right): 7.74(\mathrm{~d}, J=8.8 \mathrm{~Hz}, 2 \mathrm{H}), 7.27(\mathrm{~d}, J=8.4 \mathrm{~Hz}, 2 \mathrm{H}), 7.04(\mathrm{~d}, J=8.8$ $\mathrm{Hz}, 2 \mathrm{H}), 6.90(\mathrm{~d}, J=8.8 \mathrm{~Hz}, 2 \mathrm{H}), 3.83(\mathrm{~s}, 3 \mathrm{H}), 3.81(\mathrm{~d}, J=11.2 \mathrm{~Hz}, 6 \mathrm{H}) .{ }^{31} \mathrm{P}$ NMR $(161.9 \mathrm{MHz}$, $\mathrm{CDCl}_{3}$ ): 9.71. HRMS for $\mathrm{C}_{17} \mathrm{H}_{18} \mathrm{IN}_{3} \mathrm{O}_{4} \mathrm{P}^{+}$: calcd. $[\mathrm{M}+\mathrm{H}]^{+}:$486.0074, found: 486.0064. FTIR (cm $\left.{ }^{1}\right)$ : 3678, 3399, 3020, 2400, 1609, 1522, 1404, 1216, 1043, 929, 669, 627.

\section{Compound 6k: Dimethyl (1-(4-iodophenyl)-5-(p-tolyl)-1H-1,2,3-triazol-4-yl)phosphonate}

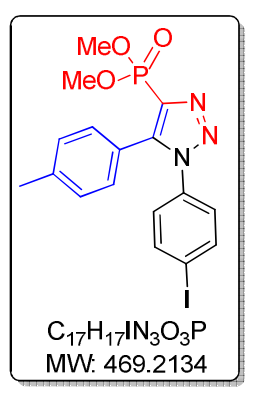

Following the general procedure, treatment of 4-methyl benzaldehyde 1t (50 mg, $0.42 \mathrm{mmol}$ ) with 4-iodoaniline $5 \mathbf{i}(137 \mathrm{mg}, 0.62 \mathrm{mmol})$ and BOR $(120 \mathrm{mg}, 0.62 \mathrm{mmol})$ in the presence of $\mathrm{K}_{2} \mathrm{CO}_{3}(86 \mathrm{mg}, 0.62 \mathrm{mmol})$ in $\mathrm{MeOH}$ $(3 \mathrm{ml})$ at room temperature for $12 \mathrm{~h}$ followed by column chromatography afforded the product $\mathbf{6 j}$ as a white solid (90 mg, 46\%). $\mathbf{R}_{\mathbf{f}}$ (Ethyl acetate/Hexane : 50/50) $=0.46 . \mathbf{M p}=127-129{ }^{\circ} \mathrm{C} .{ }^{13} \mathrm{C}$ NMR $(100 \mathrm{MHz}, \delta$ $\mathrm{ppm} / \mathrm{CDCl}_{3}$ ): $143.2\left(\mathrm{~d}, J_{\mathrm{C}-\mathrm{p}}=34.1 \mathrm{~Hz}, \mathrm{C}\right), 140.7(\mathrm{C}), 138.7(\mathrm{CH}), 138.7(\mathrm{CH})$, $135.7(\mathrm{C}), 135.3\left(\mathrm{~d}, J_{C-P}=238.9 \mathrm{~Hz}, \mathrm{C}\right), 130.0(\mathrm{CH}), 130.0(\mathrm{CH}), 129.6(\mathrm{CH}), 129.6(\mathrm{CH}), 126.8$ 
$(\mathrm{CH}), 126.8(\mathrm{CH}), 122.0(\mathrm{C}), 95.2(\mathrm{C}), 53.6\left(\mathrm{~d}, J_{C-P}=5.6 \mathrm{~Hz}, \mathrm{CH}_{3}\right), 53.6\left(\mathrm{~d}, J_{C-P}=5.6 \mathrm{~Hz}, \mathrm{CH}_{3}\right)$, $21.6\left(\mathrm{CH}_{3}\right) .{ }^{1} \mathrm{H}$ NMR $\left(400 \mathrm{MHz}, \delta \mathrm{ppm} / \mathrm{CDCl}_{3}\right): 7.72(\mathrm{~d}, J=8.8 \mathrm{~Hz}, 2 \mathrm{H}), 7.23-7.17(\mathrm{~m}, 4 \mathrm{H}), 7.02$ (d, $J=8.4 \mathrm{~Hz}, 2 \mathrm{H}), 3.79$ (d, $J=11.6 \mathrm{~Hz}, 6 \mathrm{H}), 2.37$ (s, 3H). ${ }^{31} \mathrm{P}$ NMR $\left(161.9 \mathrm{MHz}, \mathrm{CDCl}_{3}\right): 9.56$. HRMS for $\mathrm{C}_{17} \mathrm{H}_{18} \mathrm{IN}_{3} \mathrm{O}_{3} \mathrm{P}^{+}$: calcd. [M+H] $]^{+}$470.0125, found: 470.0171. FTIR (cm $\left.{ }^{-1}\right): 3853,3745$, 3684, 3019, 2957, 2854, 2400, 1602, 1518, 1490, 1216, 1038, 994, 758, 669.

Compound 6I: Dimethyl (5-(4-bromophenyl)-1-(4-iodophenyl)-1H-1,2,3-triazol-4yl)phosphonate

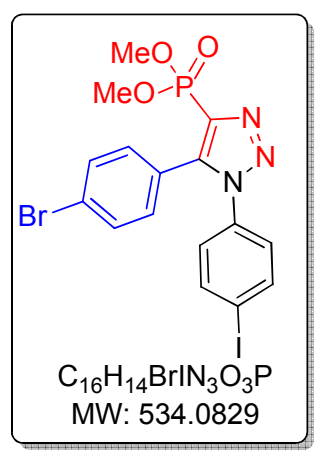

Following the general procedure, treatment of 4-bromobenzaldehyde 1u (50 mg, $0.27 \mathrm{mmol}$ ) with 4-iodo aniline $5 i$ ( $89 \mathrm{mg}, 0.40 \mathrm{mmol}$ ) and BOR (78 mg, $0.40 \mathrm{mmol})$ in the presence of $\mathrm{K}_{2} \mathrm{CO}_{3}(56 \mathrm{mg}, 0.40 \mathrm{mmol})$ in $\mathrm{MeOH}(3 \mathrm{ml})$ at room temperature for $12 \mathrm{~h}$ followed by column chromatography afforded the product $6 \mathrm{l}$ as a white solid ( $81 \mathrm{mg}, 56 \%)$. $\mathbf{R}_{\mathbf{f}}$ (Ethyl acetate/Hexane : 50/50) $=0.46 . \mathbf{M p}=170-172{ }^{\circ} \mathrm{C} .{ }^{13} \mathbf{C} \mathbf{~ N M R}$ $\left(100 \mathrm{MHz}, \delta \mathrm{ppm} / \mathrm{CDCl}_{3}\right): 142.1\left(\mathrm{~d}, J_{C-P}=33.1 \mathrm{~Hz}, \mathrm{C}\right), 138.9(\mathrm{CH}), 138.9$ $(\mathrm{CH}), 135.6\left(\mathrm{~d}, J_{\mathrm{C}-\mathrm{P}}=238.9 \mathrm{~Hz}, \mathrm{C}\right), 135.3(\mathrm{C}), 132.3(\mathrm{CH}), 132.3(\mathrm{CH})$, $131.7(\mathrm{CH}), 131.7(\mathrm{CH}), 126.8(\mathrm{CH}), 126.8(\mathrm{CH}), 125.3(\mathrm{C}), 124.03(\mathrm{C}), 95.7(\mathrm{C}), 53.8\left(\mathrm{~d}, J_{\mathrm{C}-\mathrm{p}}=\right.$ $\left.5.9 \mathrm{~Hz}, \mathrm{CH}_{3}\right), 53.8\left(\mathrm{~d}, \mathrm{~J}_{\mathrm{C}-\mathrm{P}}=5.9 \mathrm{~Hz}, \mathrm{CH}_{3}\right) .{ }^{1} \mathrm{H} \mathrm{NMR}\left(400 \mathrm{MHz}, \delta \mathrm{ppm} / \mathrm{CDCl}_{3}\right): 7.76(\mathrm{~d}, J=8.8 \mathrm{~Hz}$, $2 \mathrm{H}), 7.54(\mathrm{~d}, J=8.4 \mathrm{~Hz}, 2 \mathrm{H}), 7.22(\mathrm{~d}, J=8.8 \mathrm{~Hz}, 2 \mathrm{H}), 7.01(\mathrm{~d}, J=8.4 \mathrm{~Hz}, 2 \mathrm{H}), 3.83$ (d, $J=11.2$ $\mathrm{Hz}, 6 \mathrm{H}) .{ }^{31} \mathrm{P}$ NMR (161.9 MHz, $\left.\mathrm{CDCl}_{3}\right)$ : 8.94. HRMS for $\mathrm{C}_{16} \mathrm{H}_{15} \mathrm{BrIN}_{3} \mathrm{O}_{3} \mathrm{P}^{+}$: calcd. $[\mathrm{M}+\mathrm{H}]^{+}$: 533.9074, found: 533.9124. FTIR ( $\left.\mathrm{cm}^{-1}\right): 3683,3020,2956,2855,2401,1606,1487,1411$, 1216, 1046, 927, 763, 671, 626, 574.

Compound 6m: Dimethyl (5-(furan-2-yl)-1-(4-iodophenyl)-1H-1,2,3-triazol-4yl)phosphonate

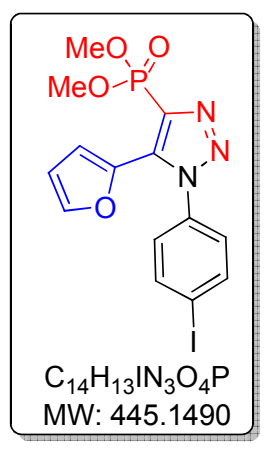

Following the general procedure, treatment of furfural $1 \mathrm{~m}(50 \mathrm{mg}, 0.52$ mmol) with 4-iodoaniline $5 \mathbf{i}(171 \mathrm{mg}, 0.78 \mathrm{mmol})$ and BOR (150 mg, 0.78 $\mathrm{mmol})$ in the presence of $\mathrm{K}_{2} \mathrm{CO}_{3}(108 \mathrm{mg}, 0.78 \mathrm{mmol})$ in $\mathrm{MeOH}(3 \mathrm{ml})$ at room temperature for $12 \mathrm{~h}$ followed by column chromatography afforded the product $6 \mathrm{~m}$ as a orange solid $(100 \mathrm{mg}, 43 \%)$. $\mathbf{R}_{\mathbf{f}}$ (Ethyl acetate/Hexane $: 50 / 50)=0.46 . \mathrm{Mp}=133-135{ }^{\circ} \mathrm{C} .{ }^{13} \mathrm{C}$ NMR $\left(100 \mathrm{MHz}, \delta \mathrm{ppm} / \mathrm{CDCl}_{3}\right)$ : $145.1(\mathrm{CH}), 138.7(\mathrm{CH}), 138.7(\mathrm{CH}), 136.3(\mathrm{C}), 135.4(\mathrm{C}), 134.2$ (d, $J_{C-P}=$ $32.0 \mathrm{~Hz}, \mathrm{C}) 134.3(\mathrm{C}), 127.1(\mathrm{CH}), 127.1(\mathrm{CH}), 115.9(\mathrm{CH}), 112.2(\mathrm{CH}), 95.9$ (C), $54.0\left(\mathrm{~d}, J_{C-P}=5.9 \mathrm{~Hz}, \mathrm{CH}_{3}\right), 54.0\left(\mathrm{~d}, J_{C-P}=5.9 \mathrm{~Hz}, \mathrm{CH}_{3}\right) .{ }^{1} \mathrm{H} \mathrm{NMR}\left(400 \mathrm{MHz}, \delta \mathrm{ppm} / \mathrm{CDCl}_{3}\right)$ : $7.84(\mathrm{dd}, J=6.8,2.0 \mathrm{~Hz}, 2 \mathrm{H}), 7.45(\mathrm{~d}, J=1.2 \mathrm{~Hz}, 1 \mathrm{H}), 7.14(\mathrm{dd}, J=8.8,2.0 \mathrm{~Hz}, 2 \mathrm{H}), 7.08(\mathrm{~d}, J=$ $1.6 \mathrm{~Hz}, 1 \mathrm{H}), 6.51(\mathrm{dd}, J=3.6,2.0 \mathrm{~Hz}, 1 \mathrm{H}), 3.91(\mathrm{~d}, J=11.6 \mathrm{~Hz}, 6 \mathrm{H}) .{ }^{31} \mathbf{P}$ NMR $(161.9 \mathrm{MHz}$, $\mathrm{CDCl}_{3}$ ): 8.90. HRMS for $\mathrm{C}_{14} \mathrm{H}_{14} \mathrm{IN}_{3} \mathrm{O}_{3} \mathrm{P}^{+}$: calcd. $[\mathrm{M}+\mathrm{H}]^{+}: 445.9761$, found: 445.9756. FTIR (cm $\left.{ }^{1}\right):$ 3392, 3019, 2926, 2854, 1644, 1491, 1403, 1216, 1054, 827, 771, 669, 576. 


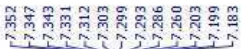

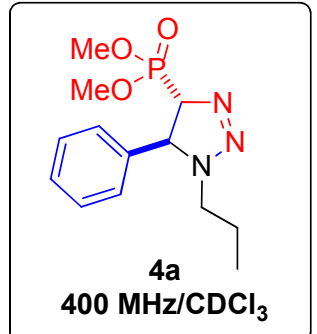


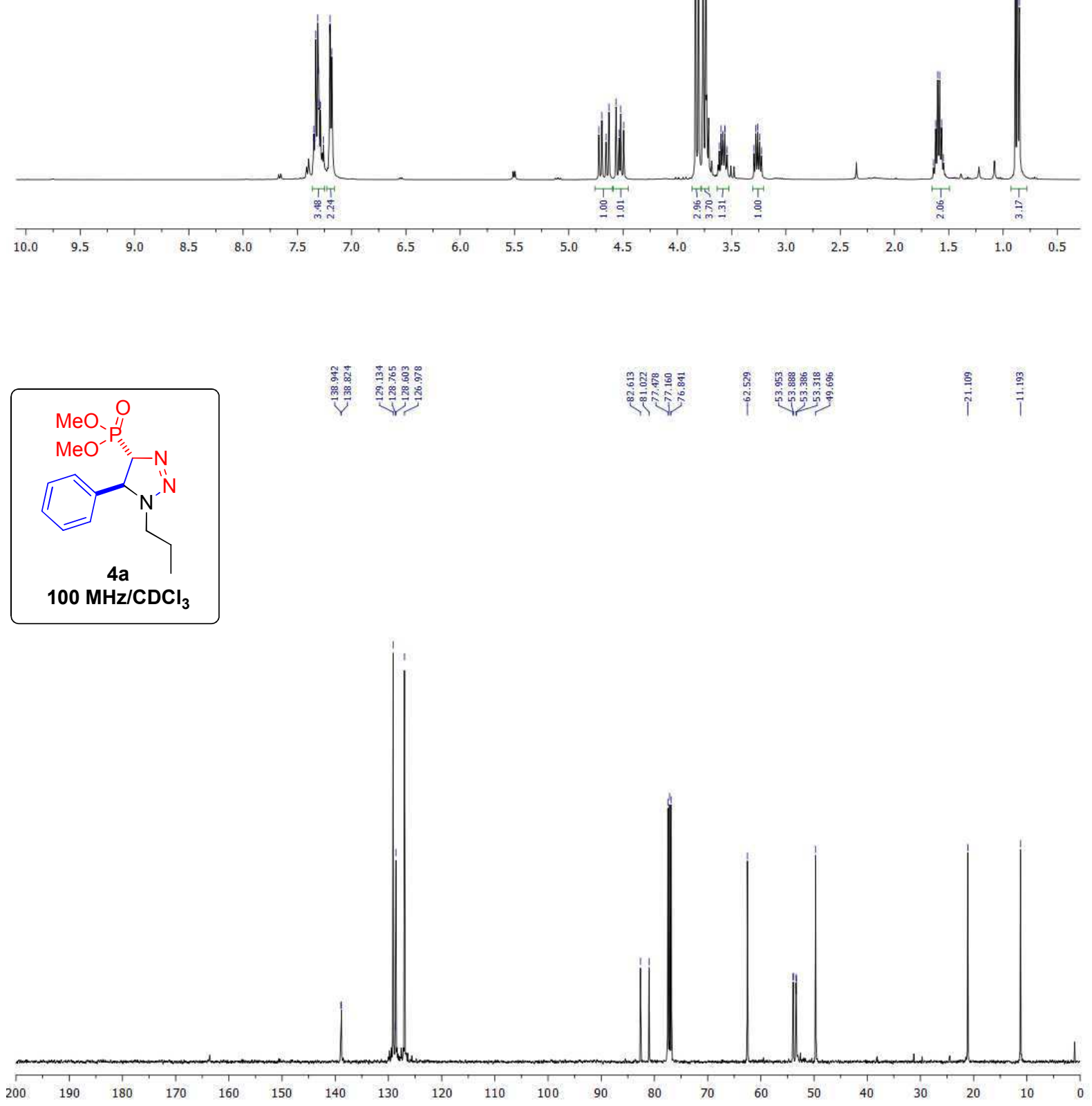

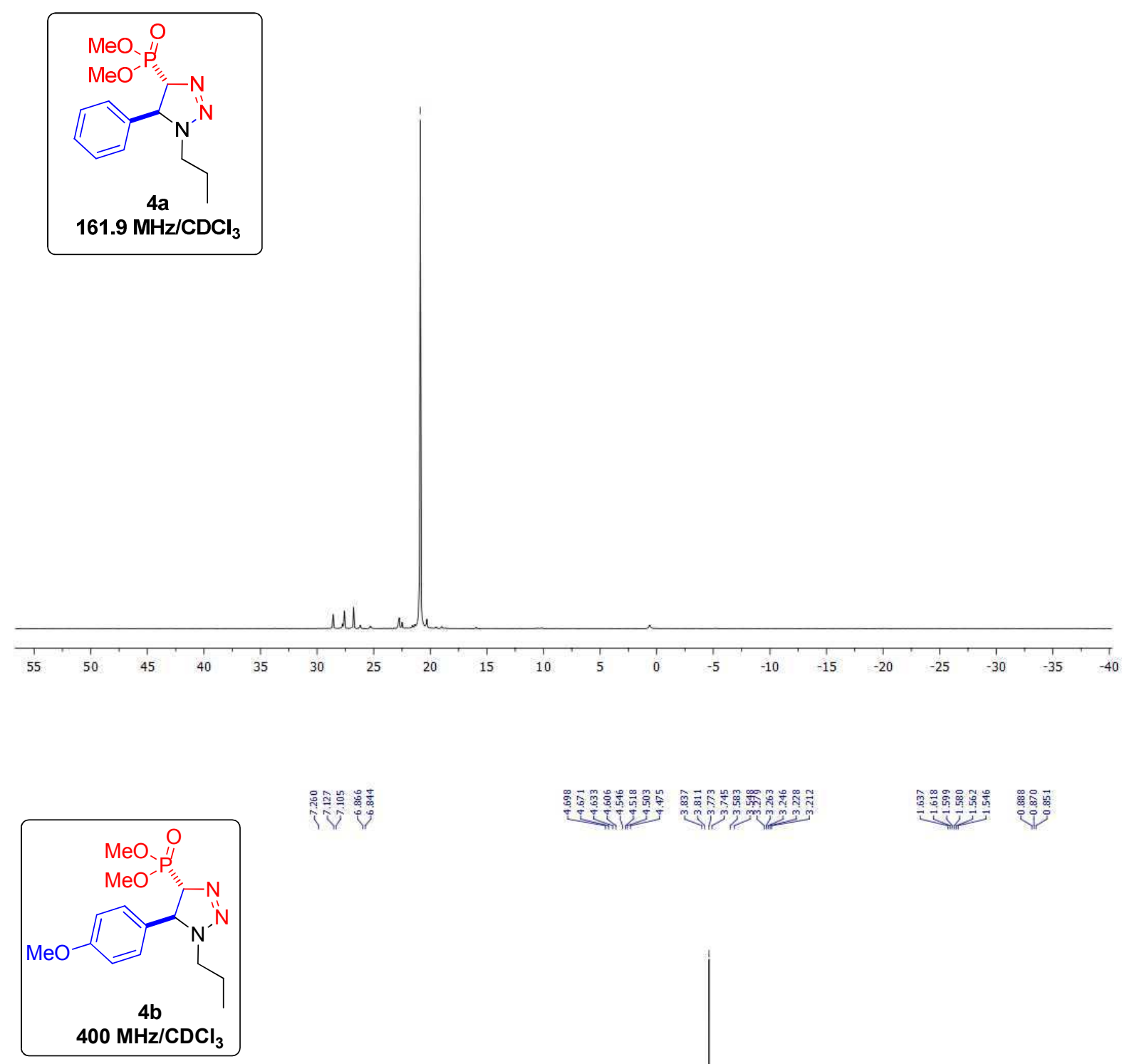

ำํํำ

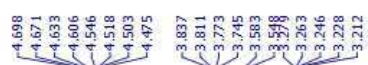

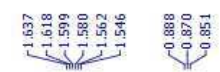

$400 \mathrm{MHz} / \mathrm{CDCl}_{3}$ 

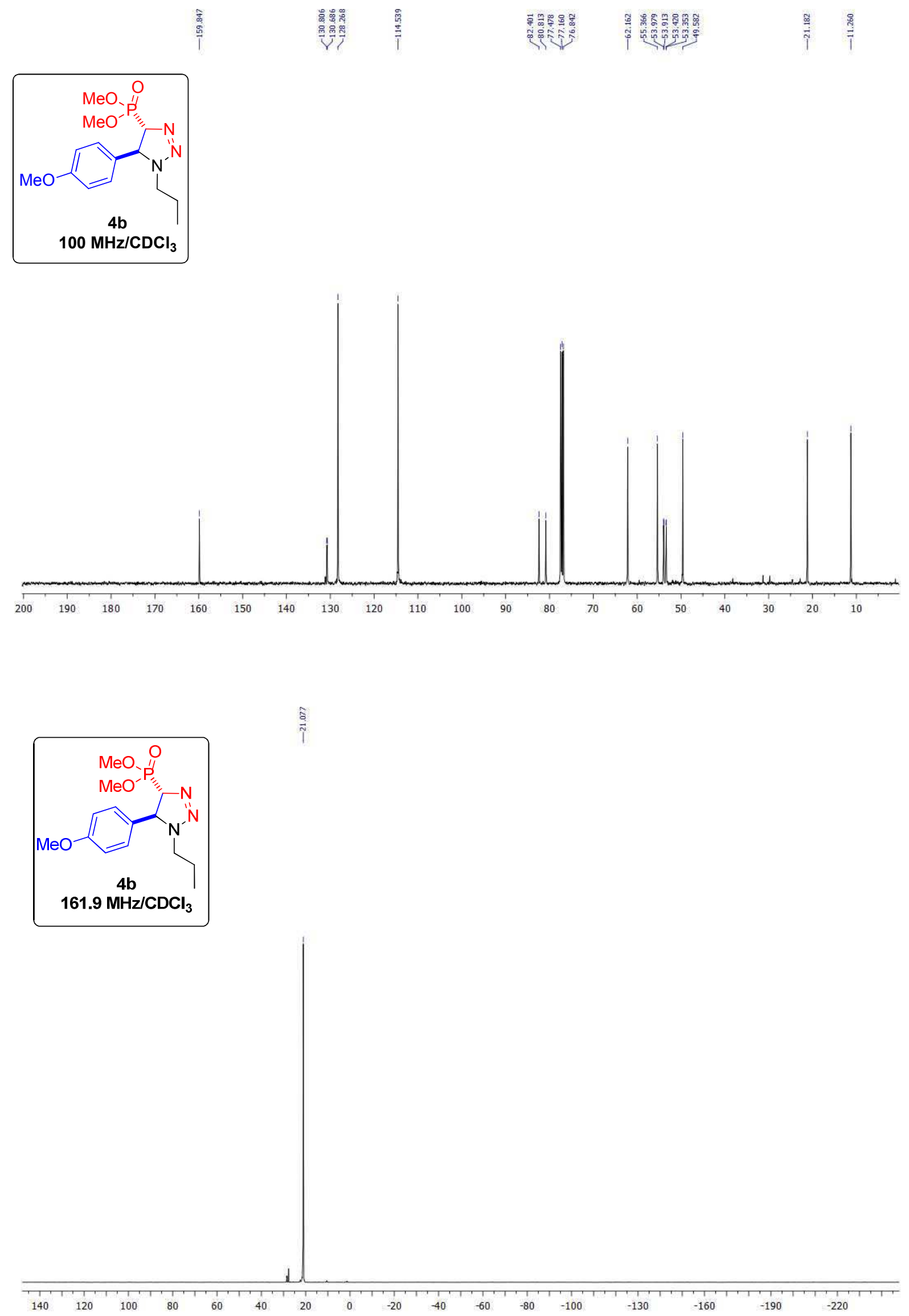

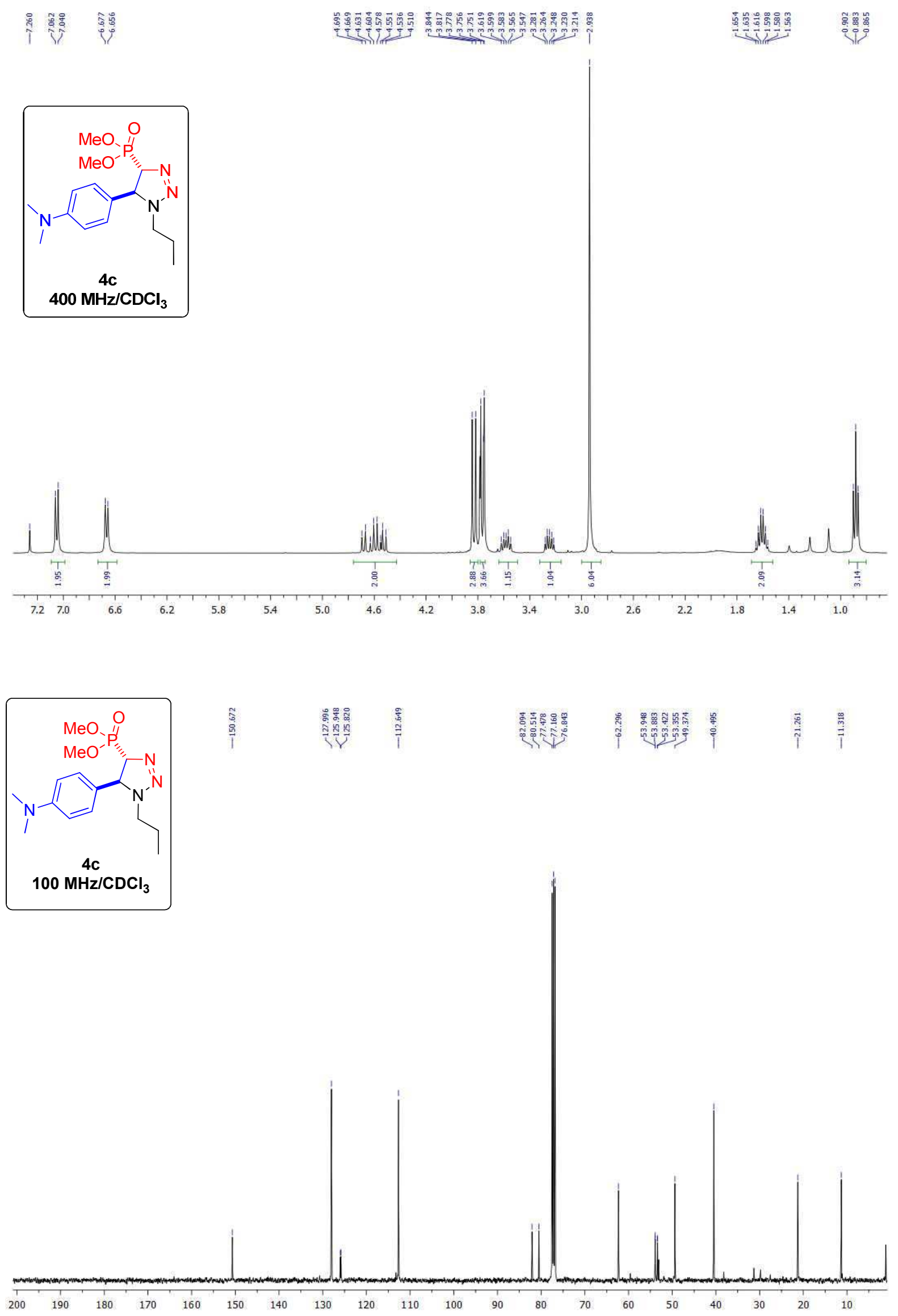

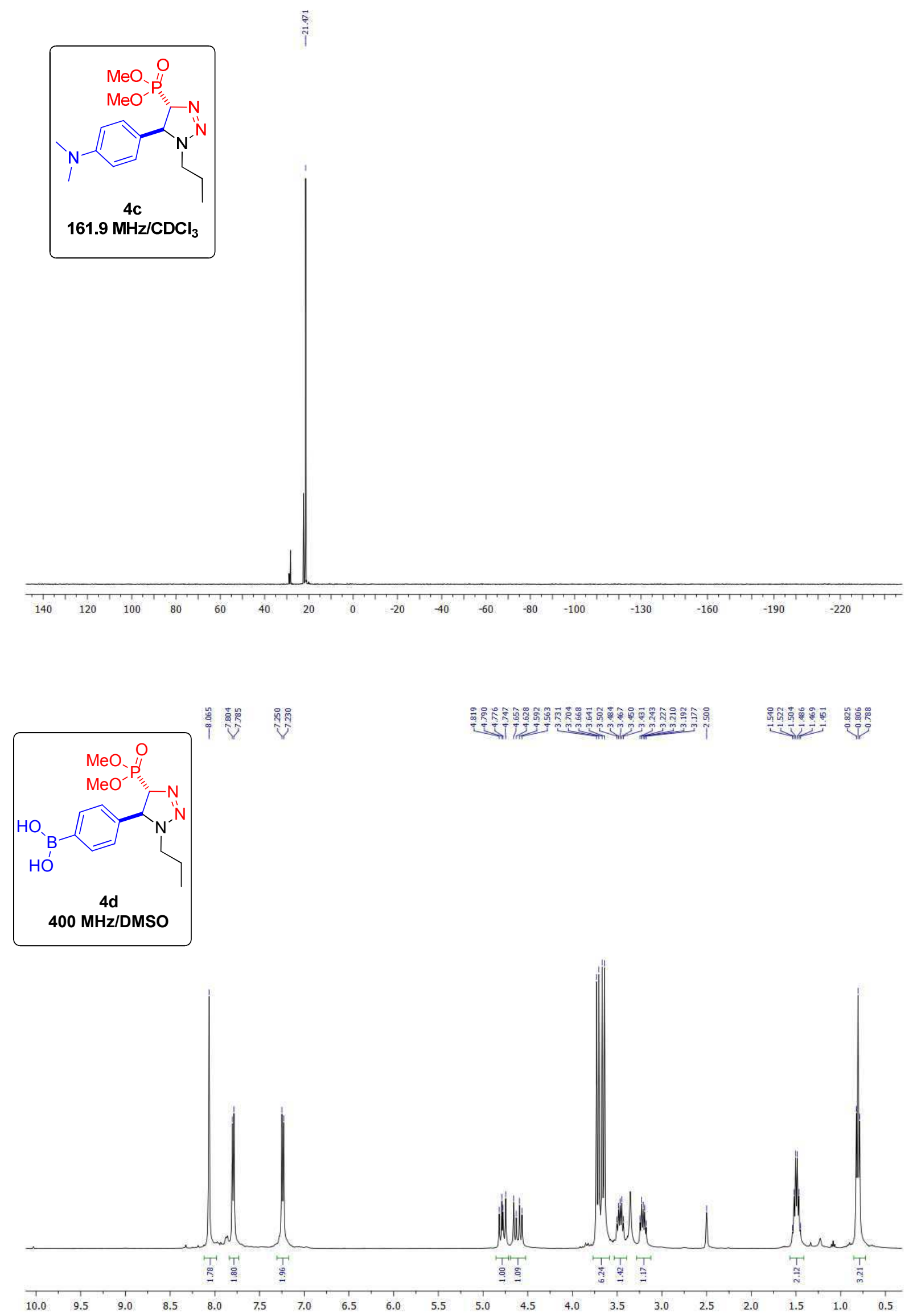


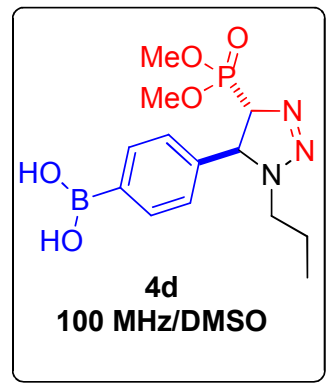

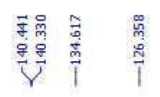

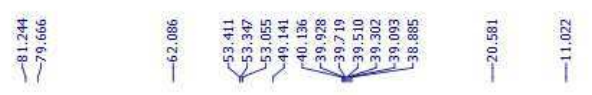
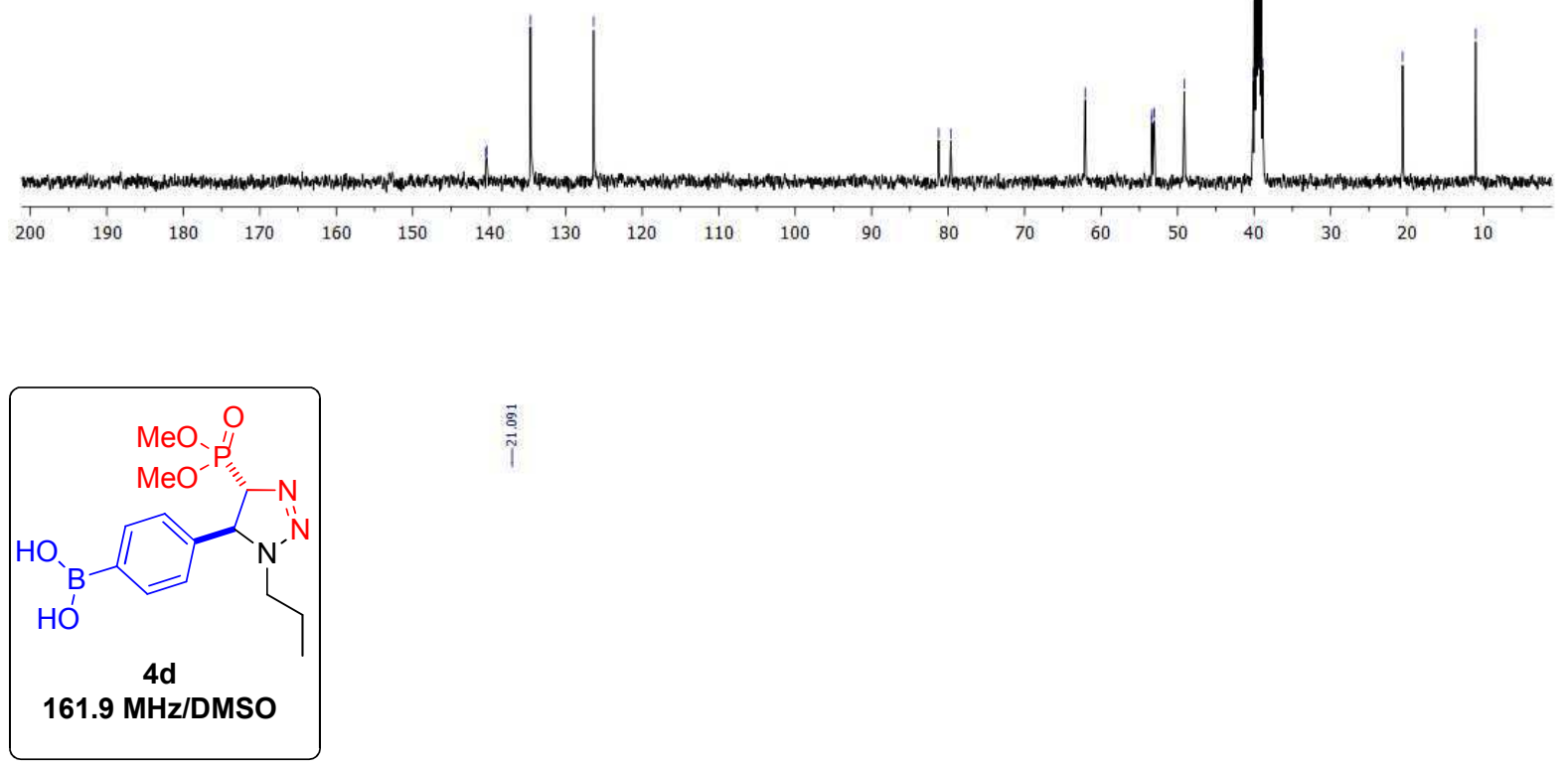

$\frac{\vec{s}}{\hat{\imath}}$

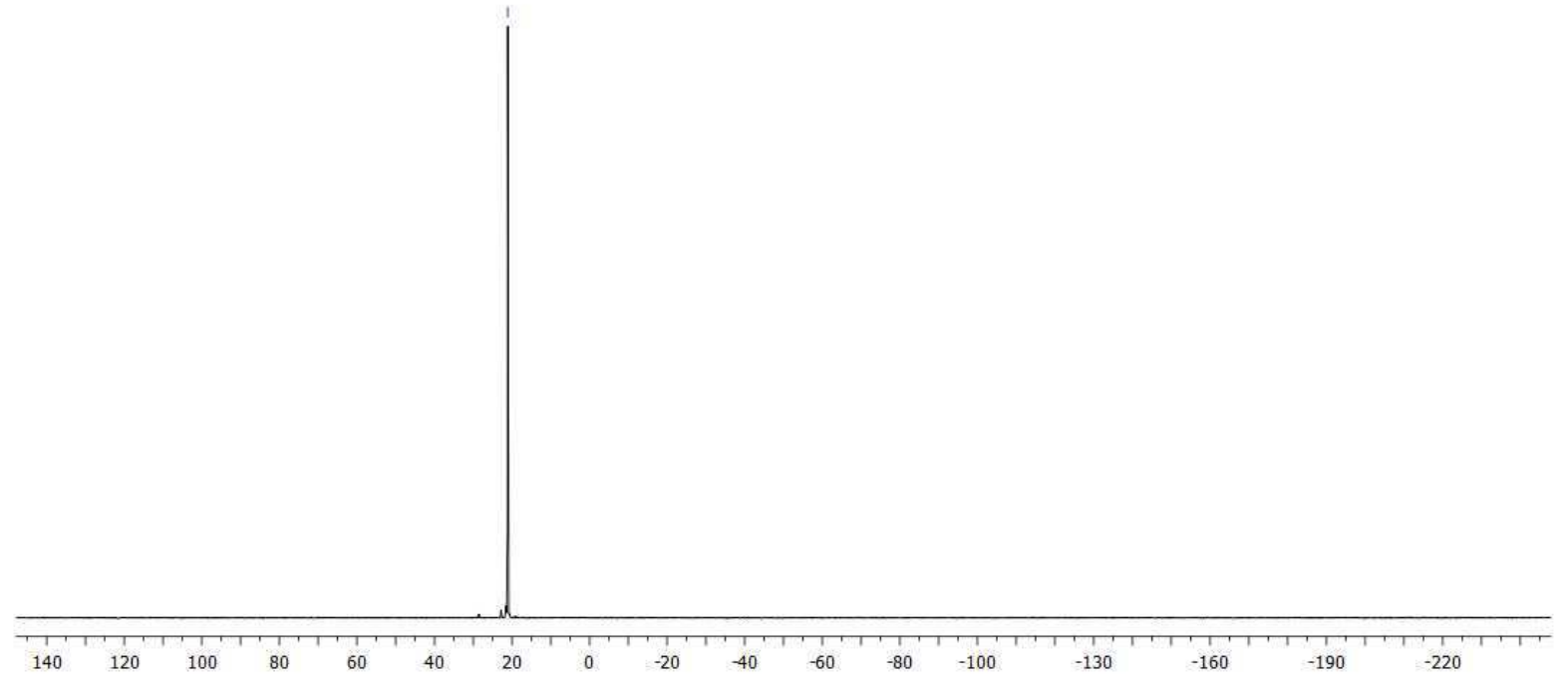



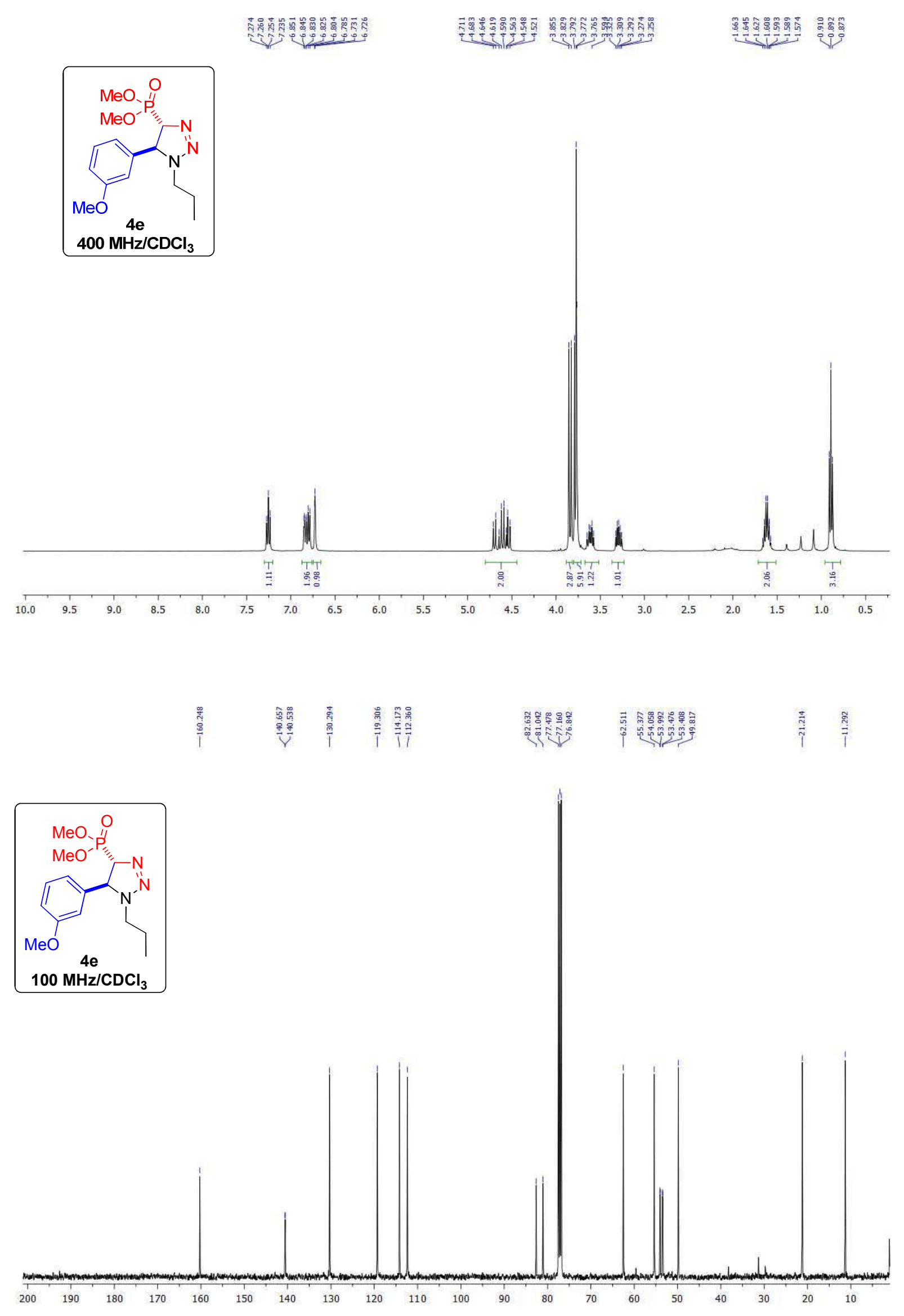


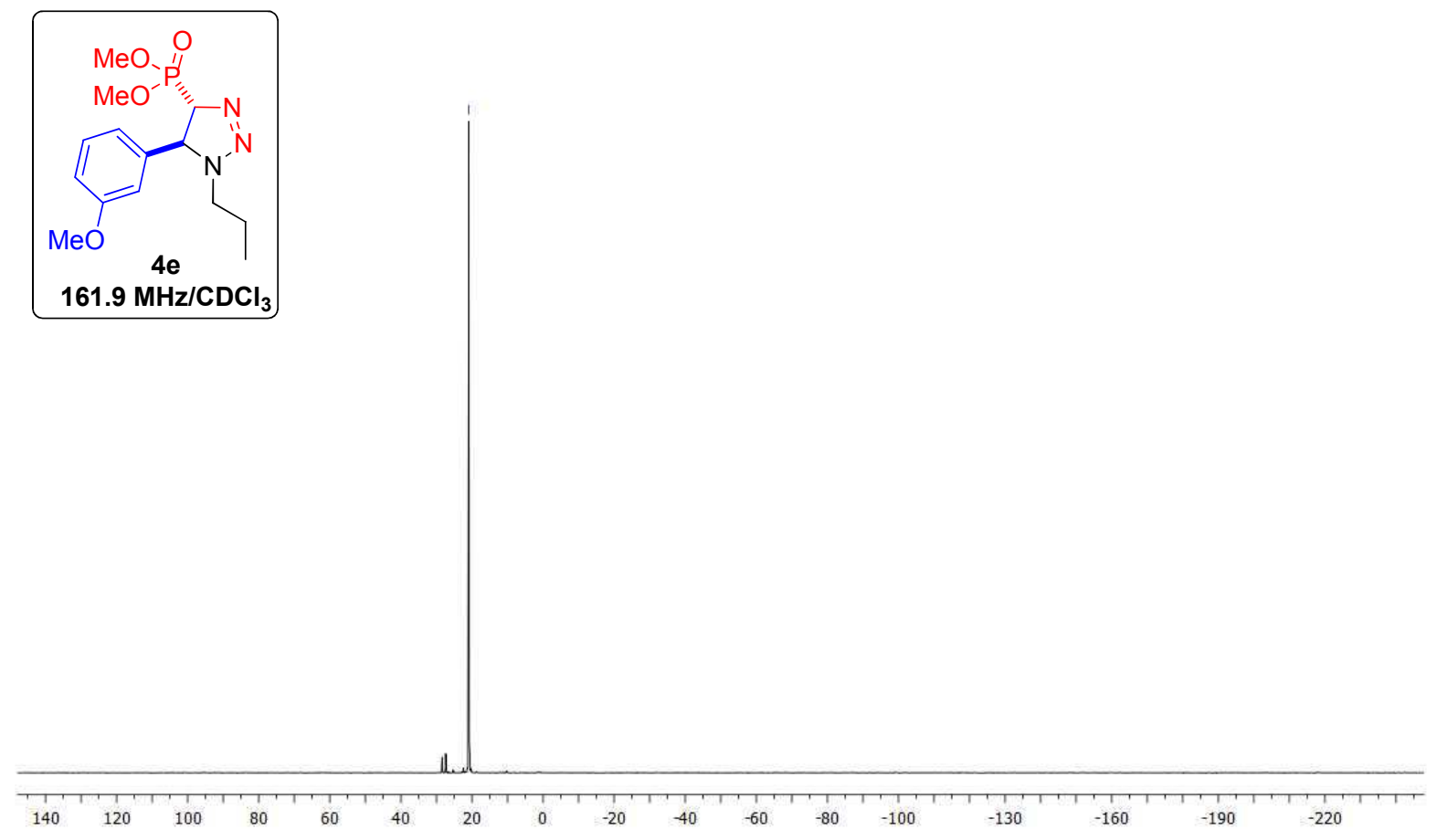

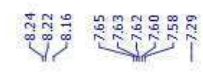
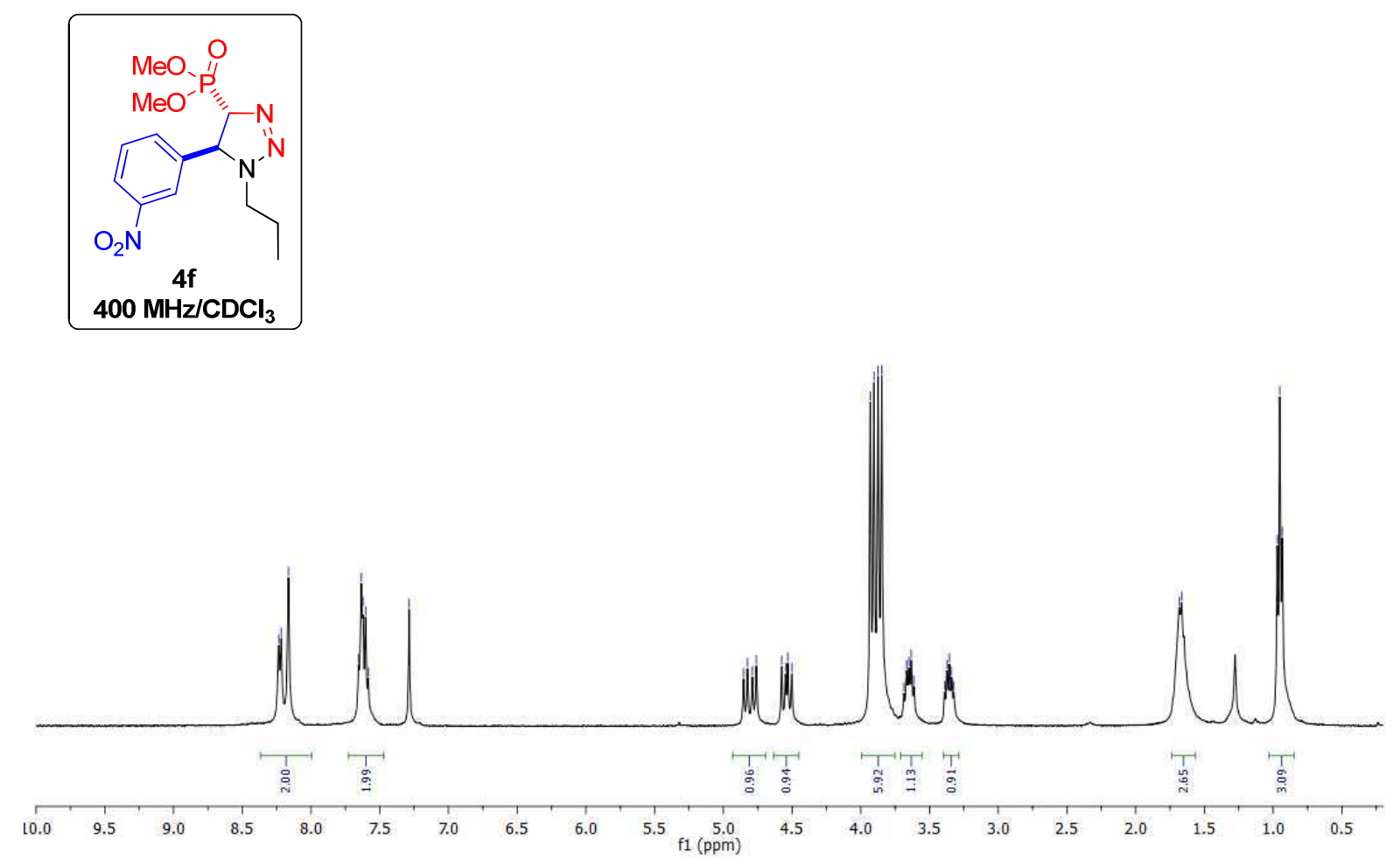

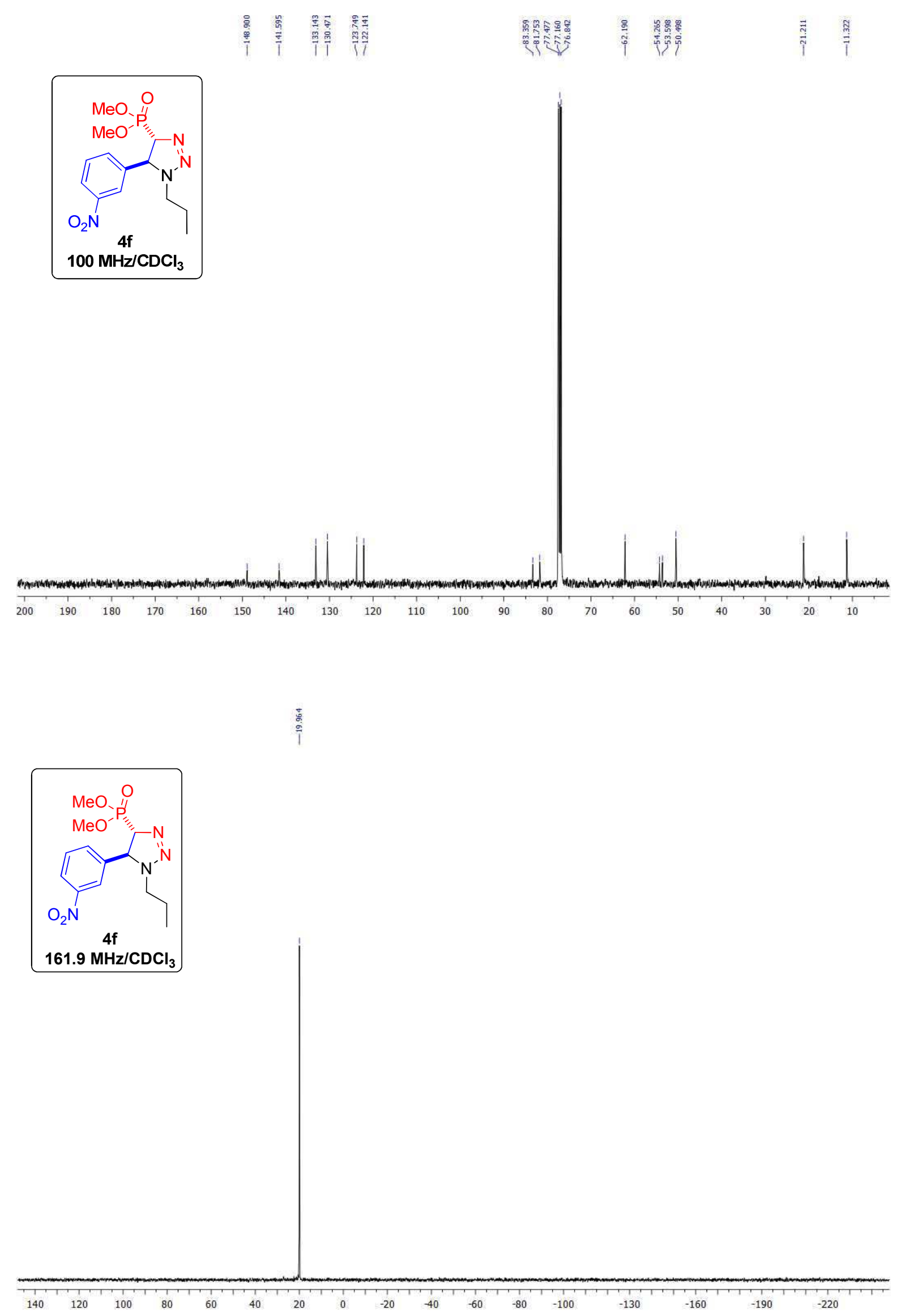

34 


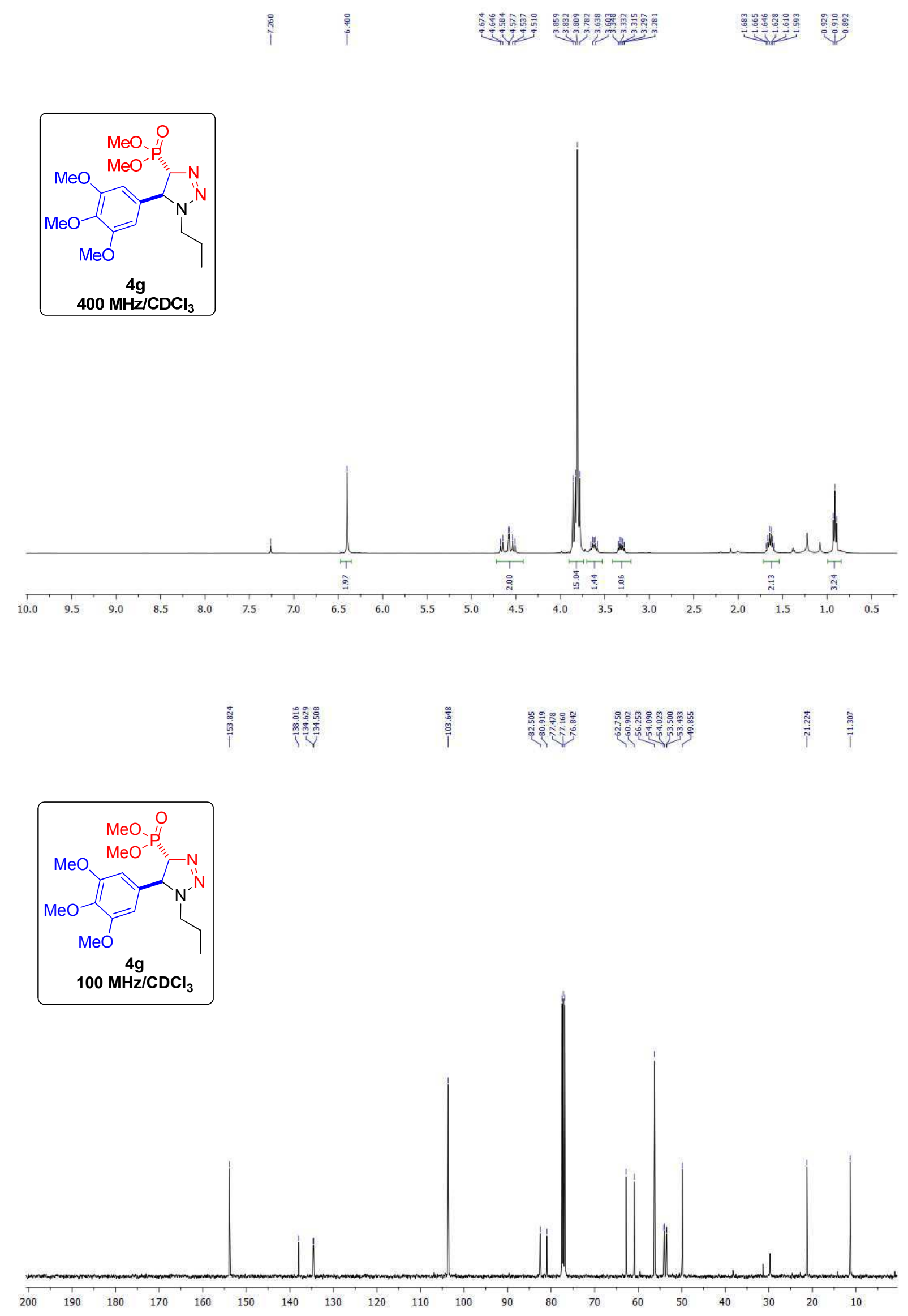



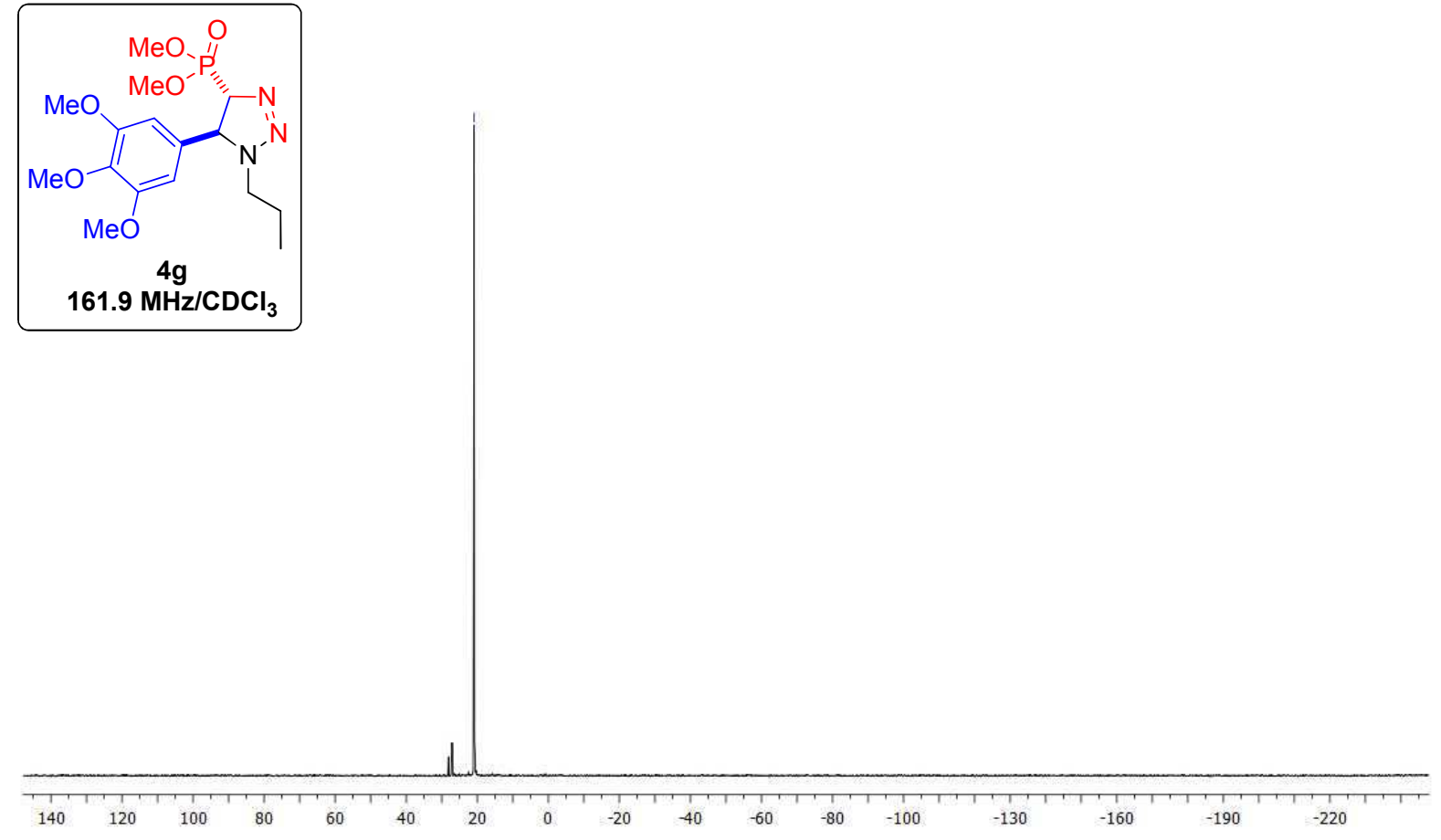

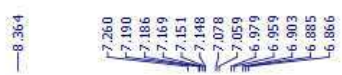

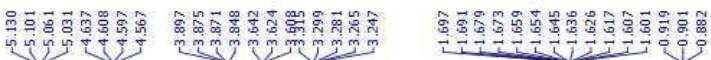
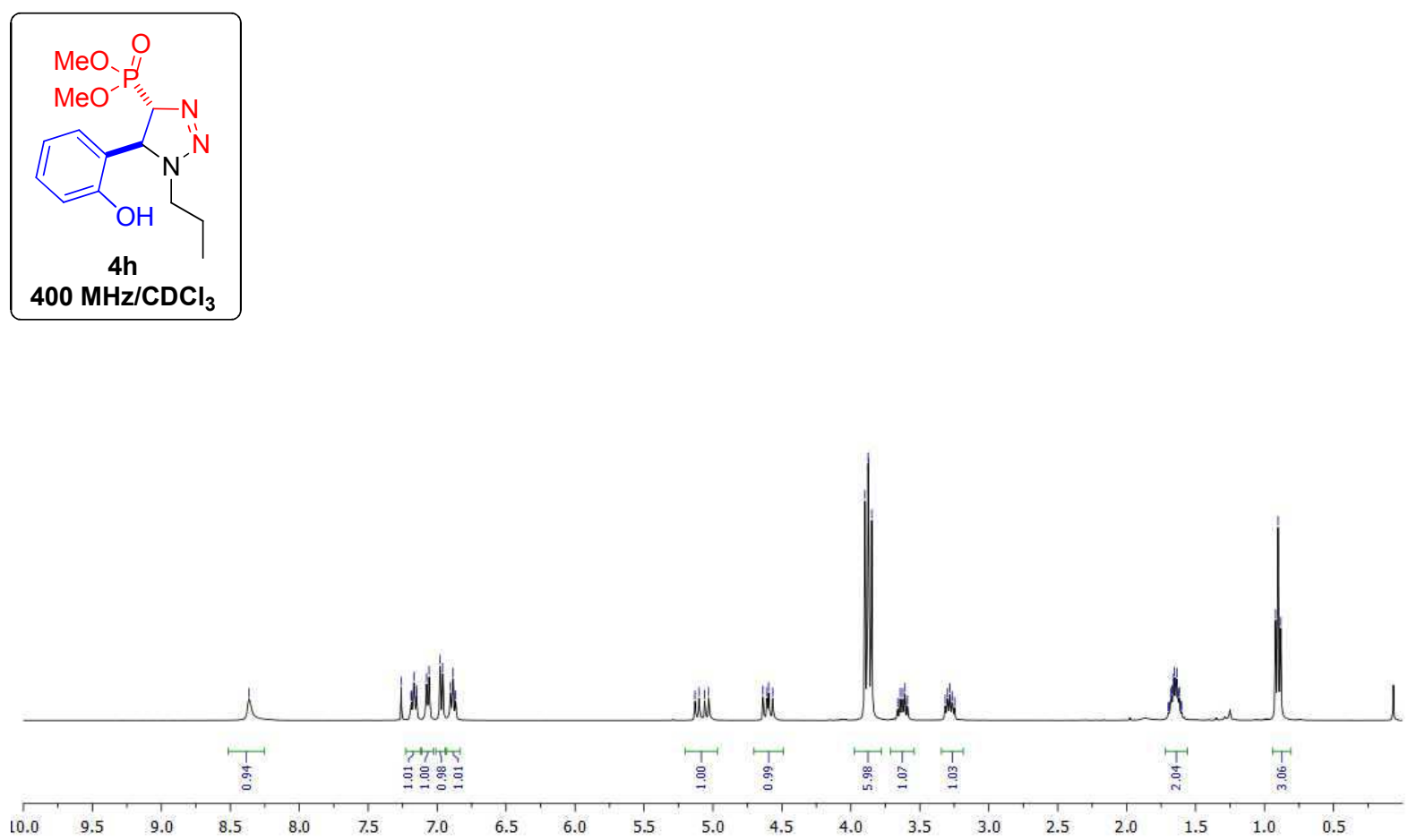


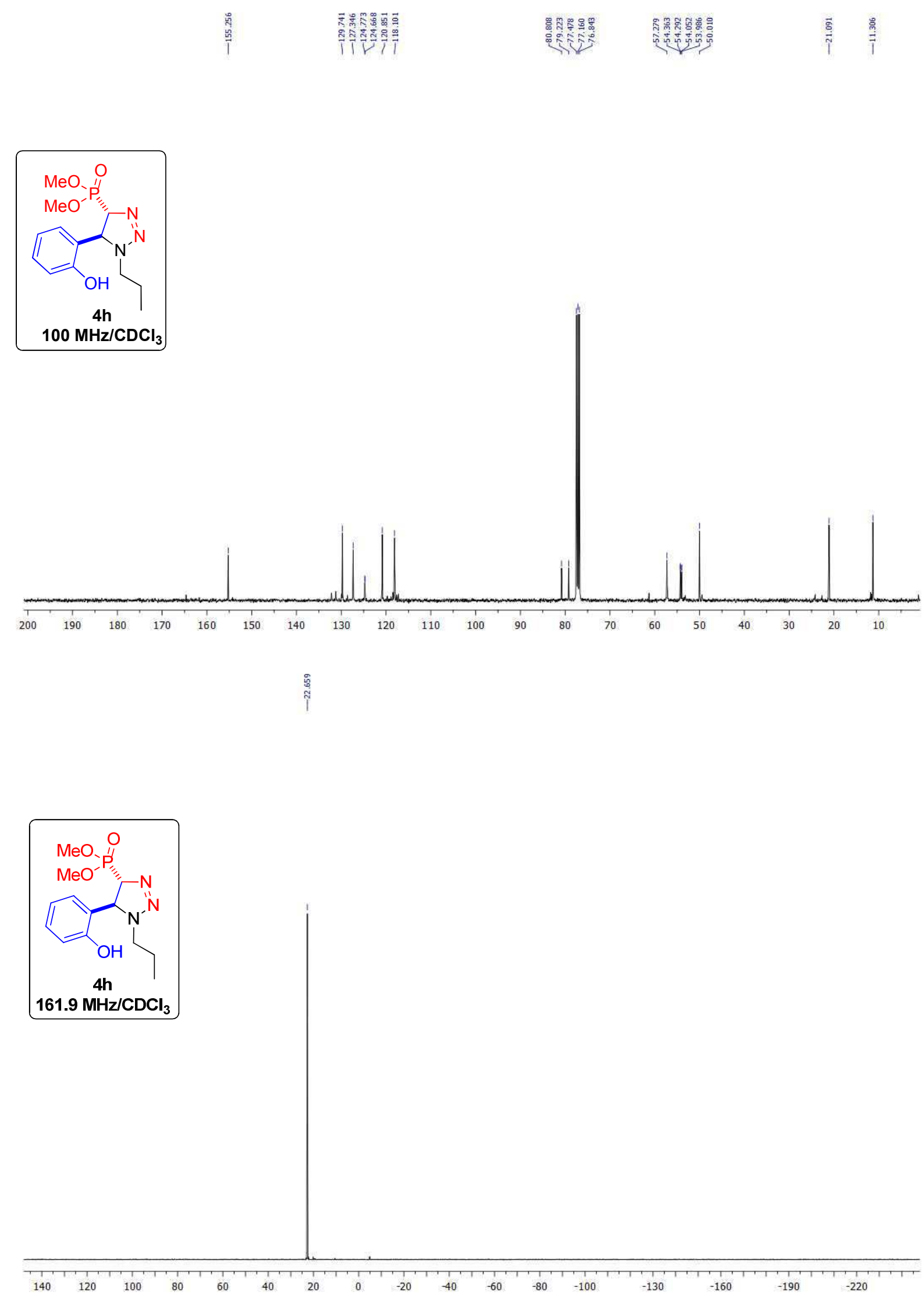




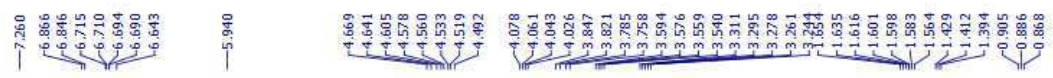
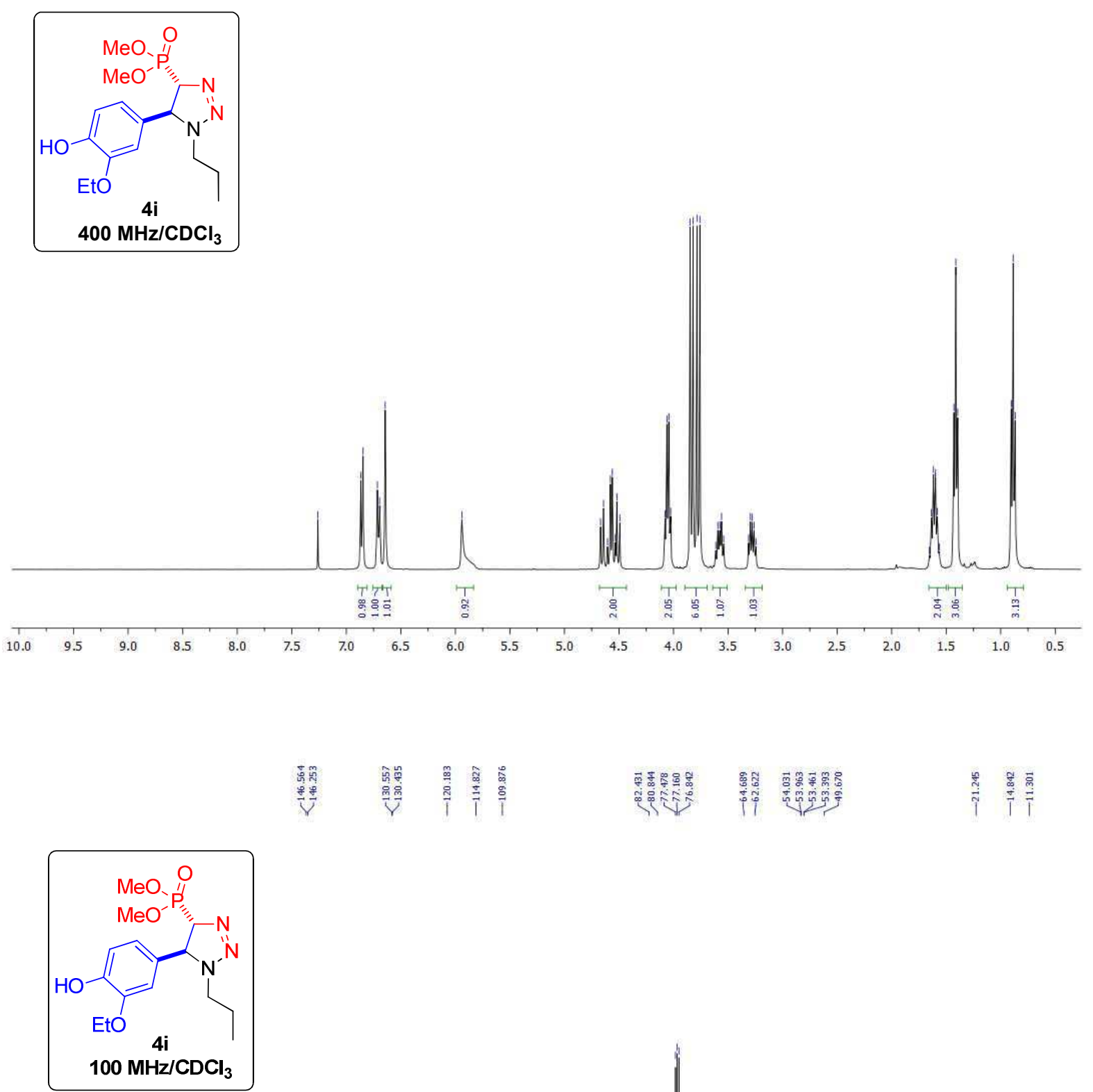

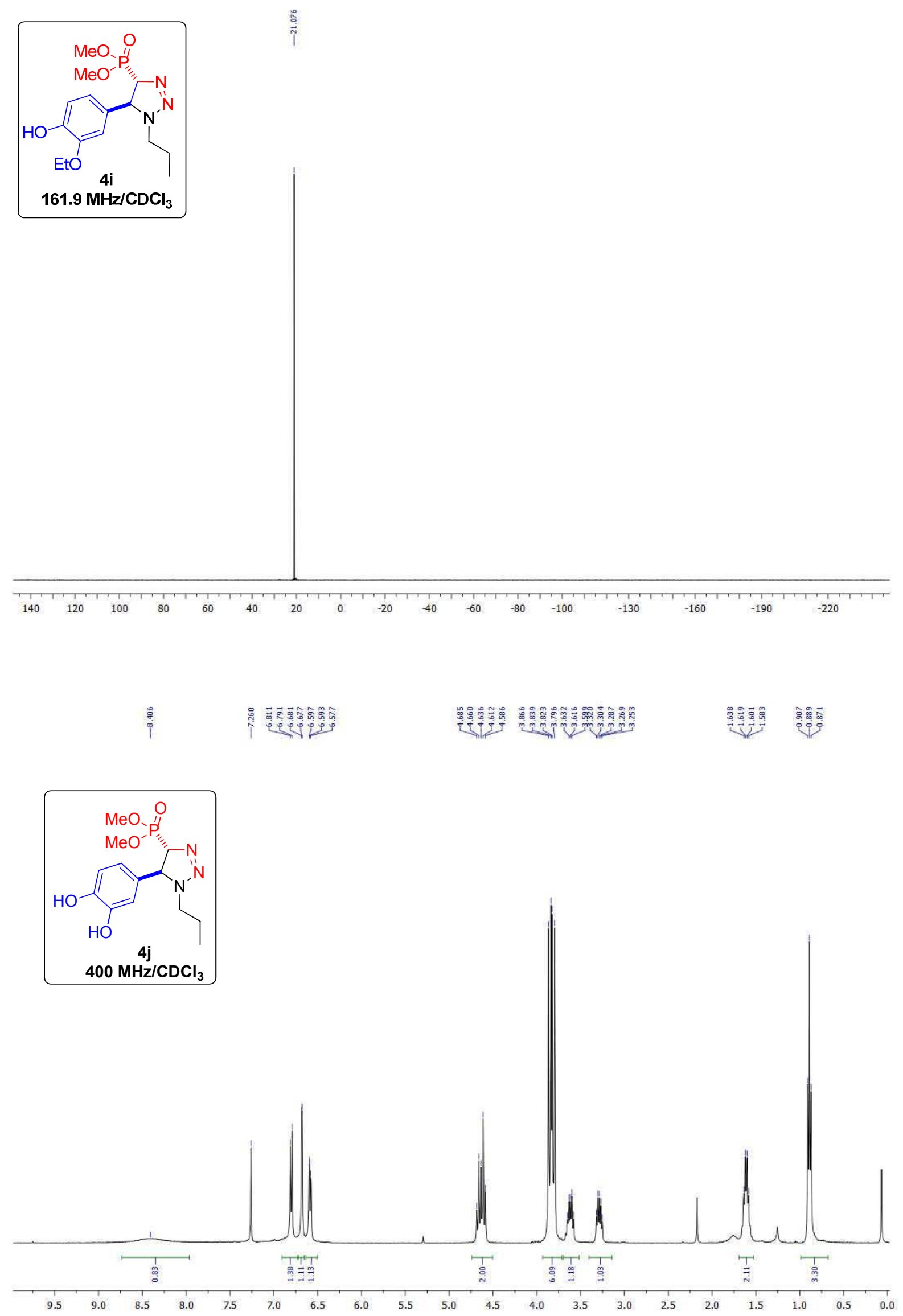

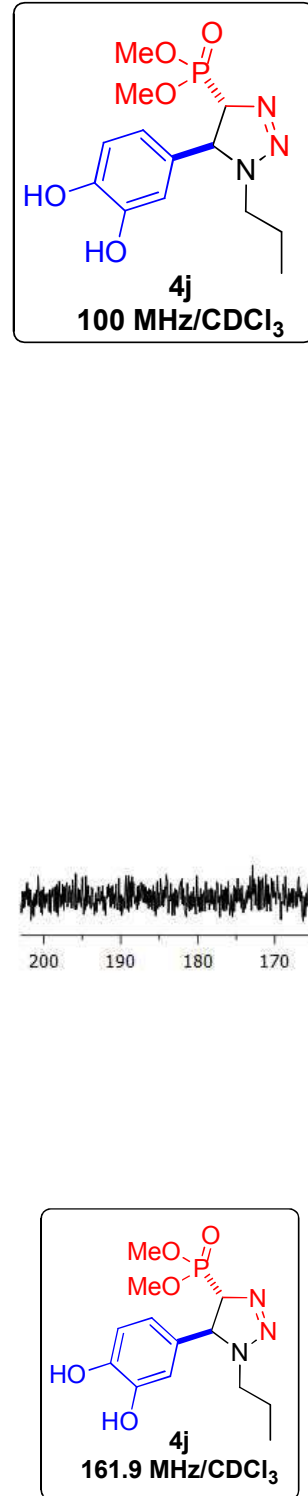

$\begin{array}{llllllllllll}140 & 120 & 100 & 80 & 60 & 40 & 20 & 0 & -20 & -40 & -60 & -80\end{array}$ 


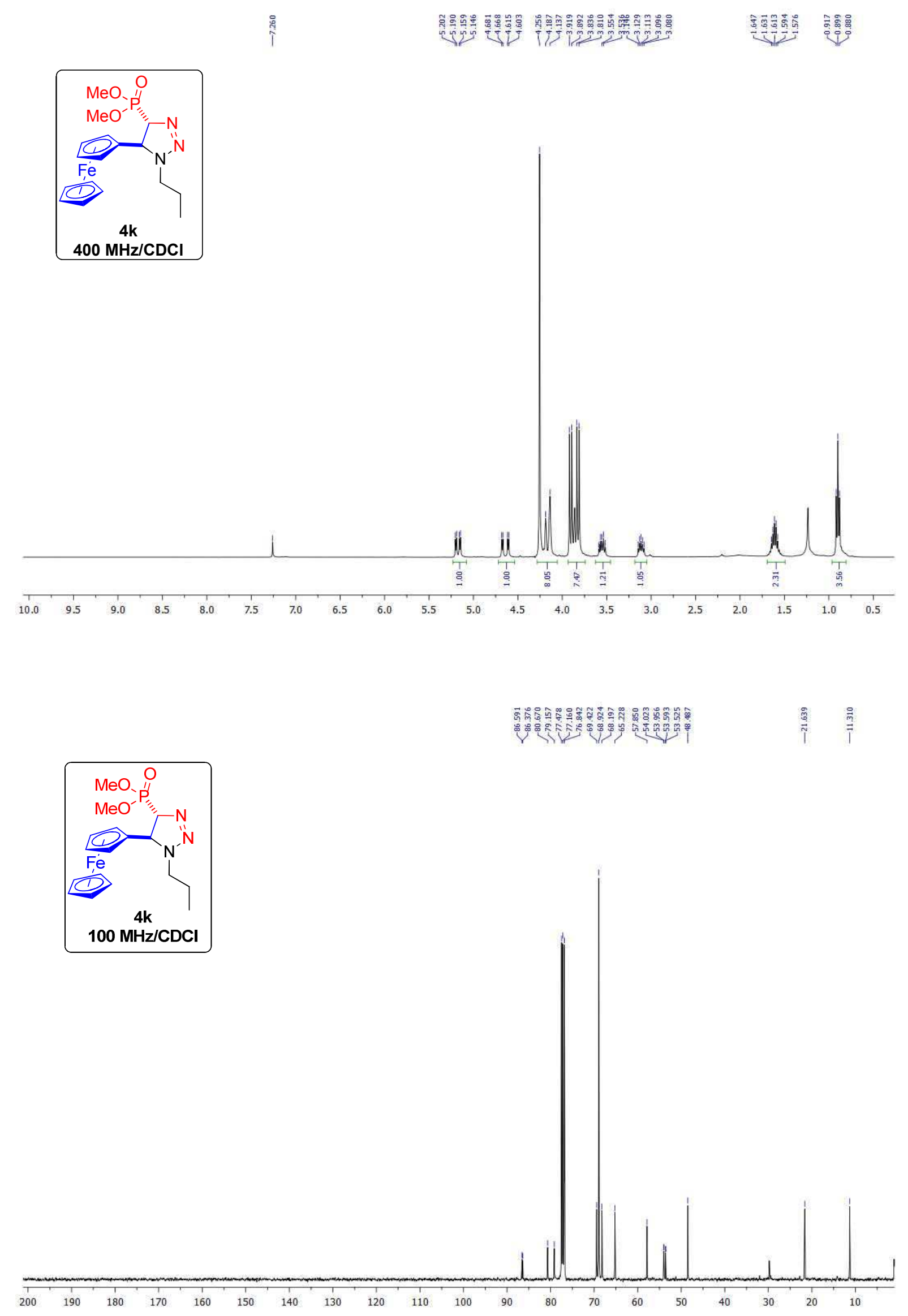




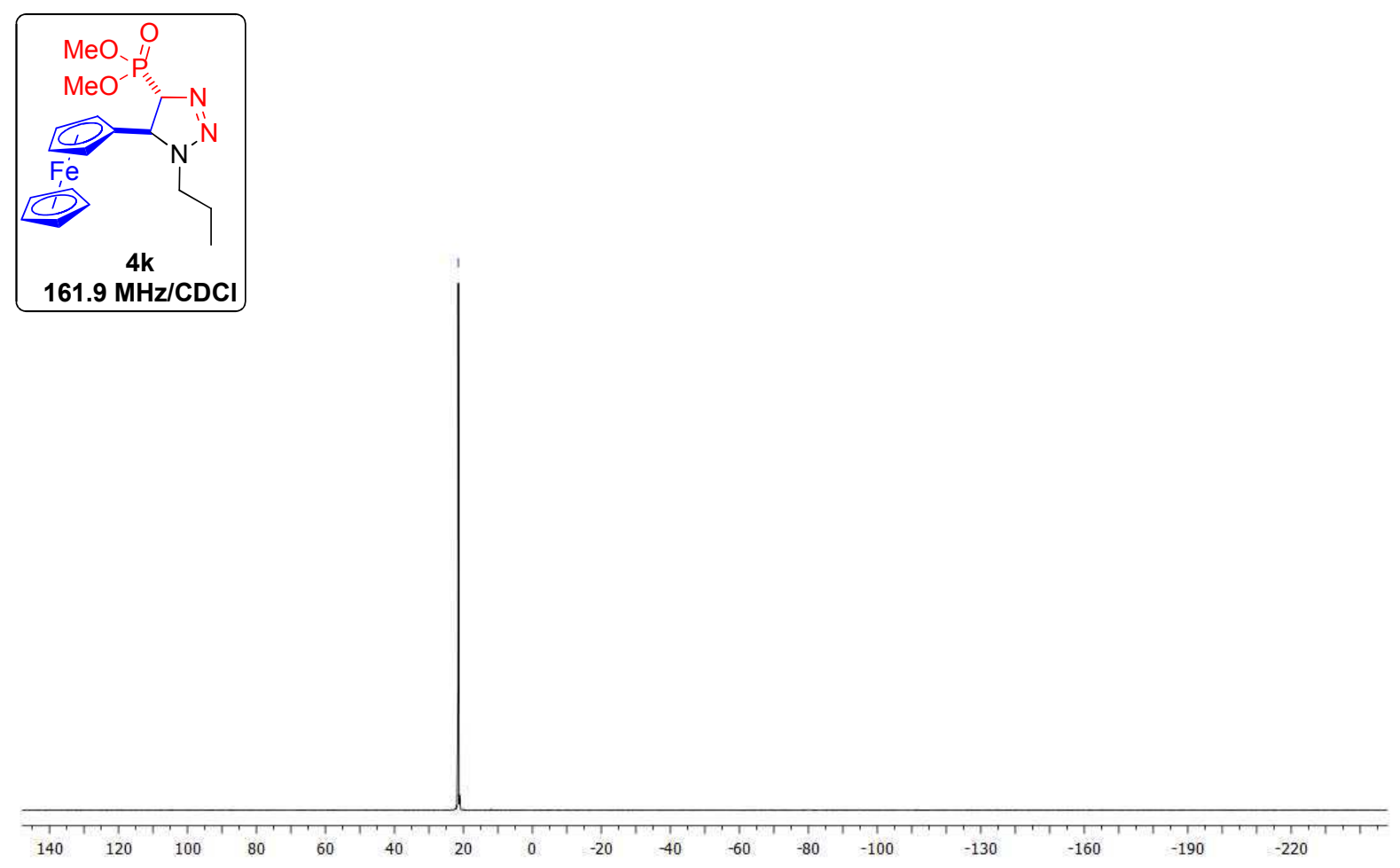

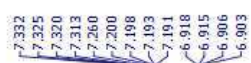

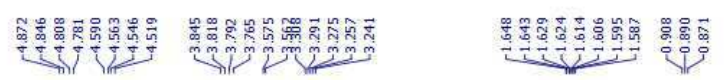
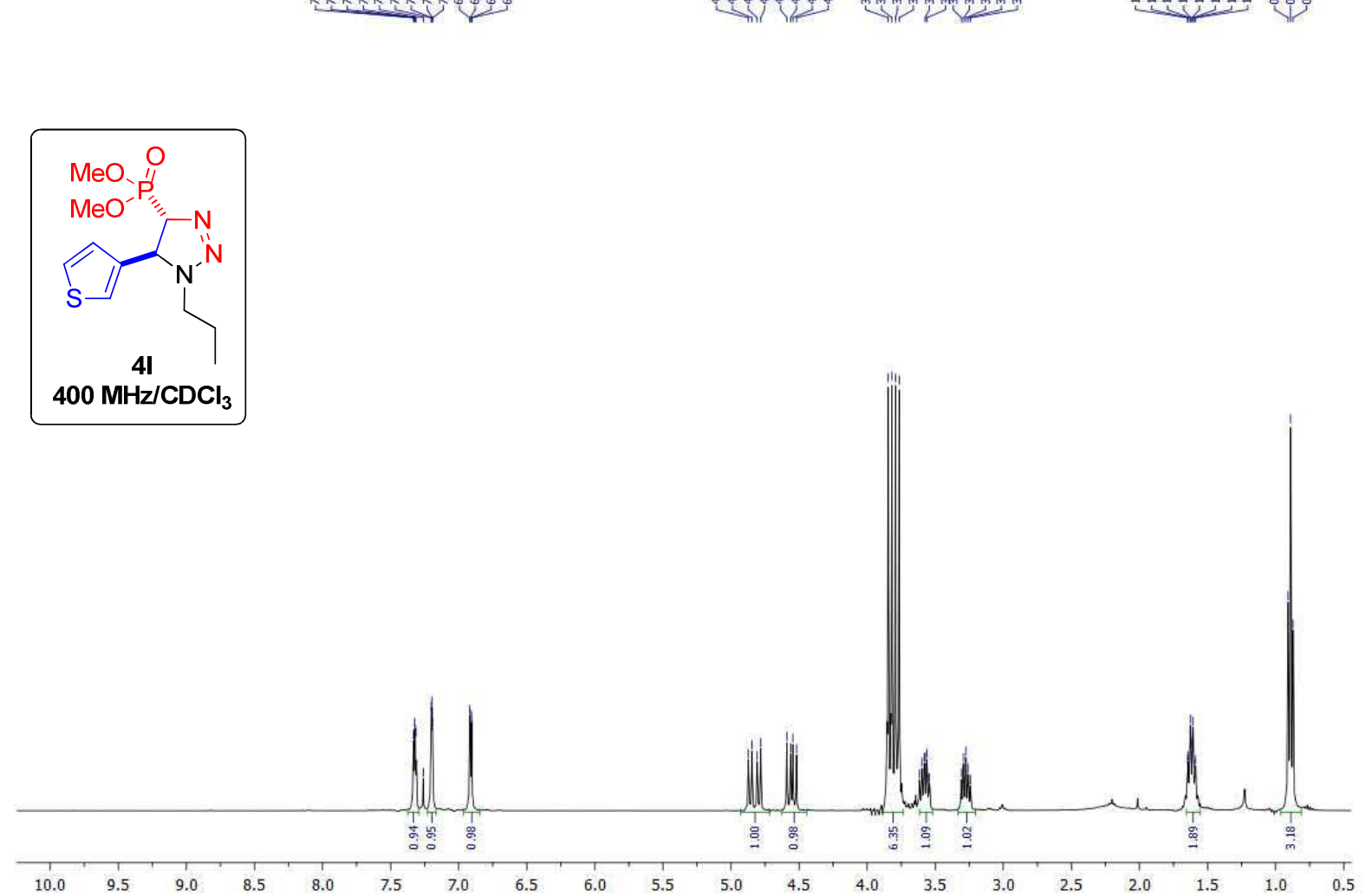


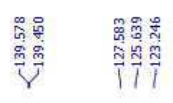

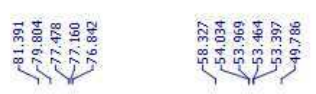

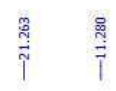
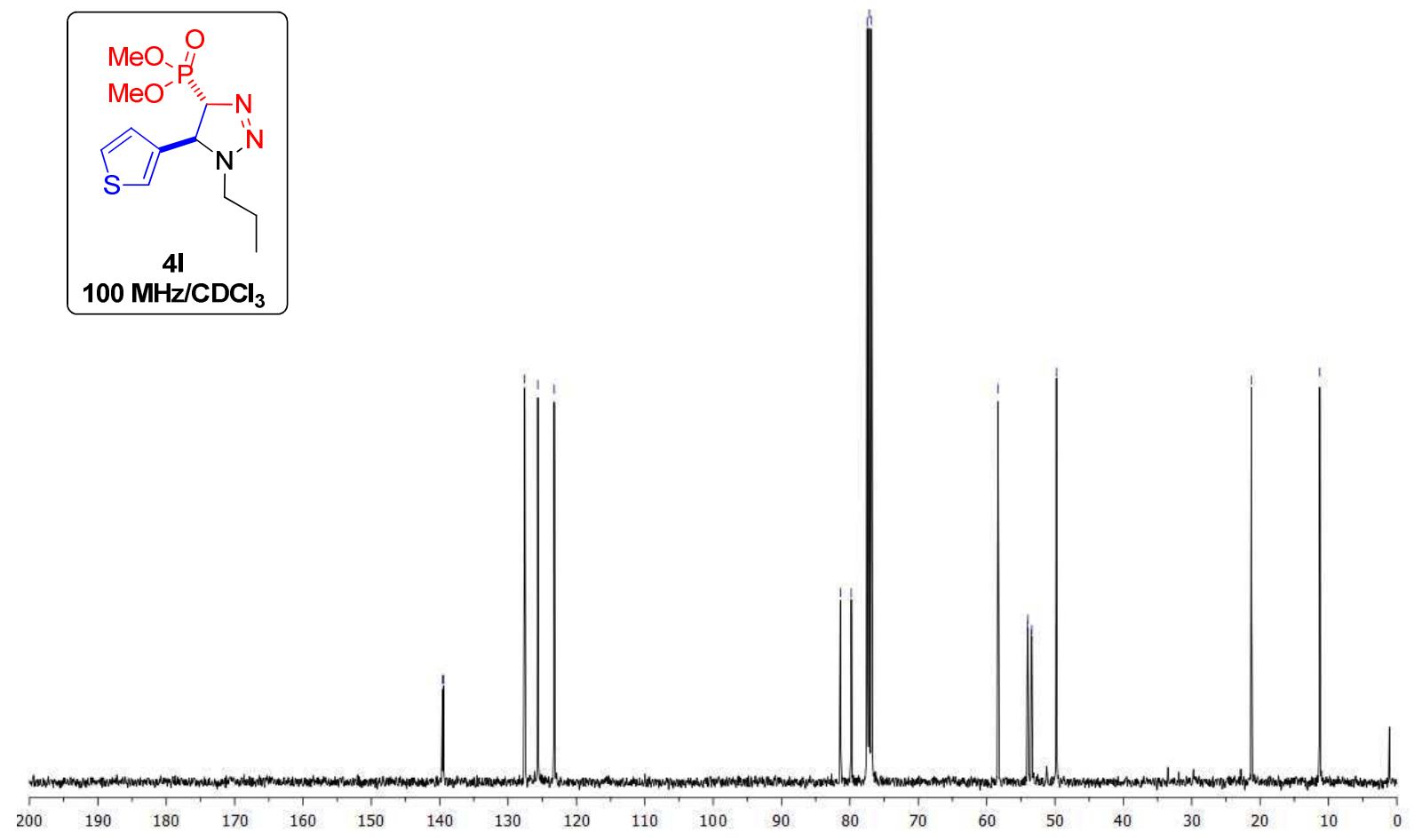

离

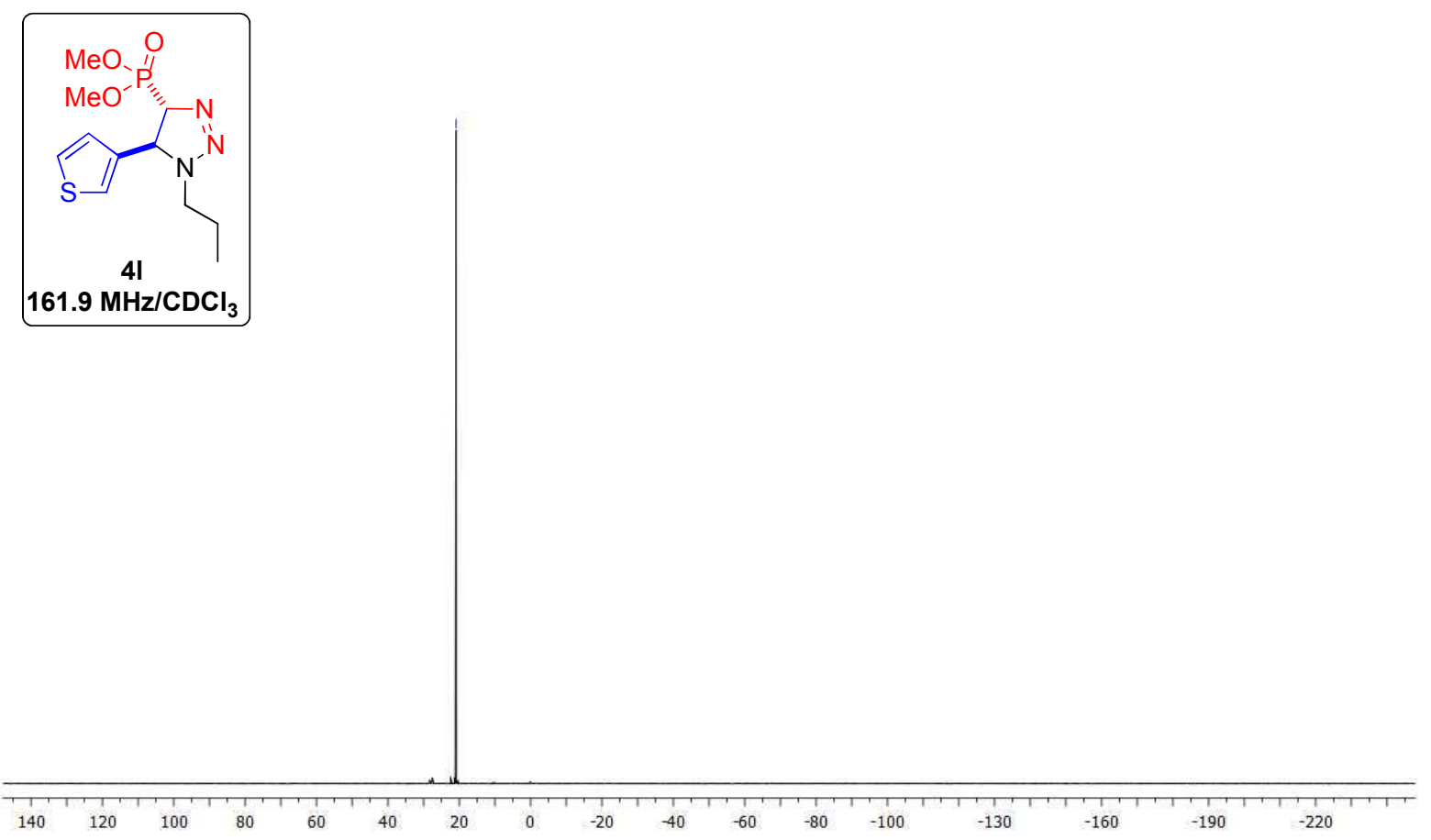

43 


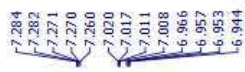

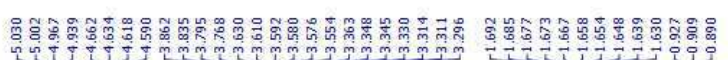
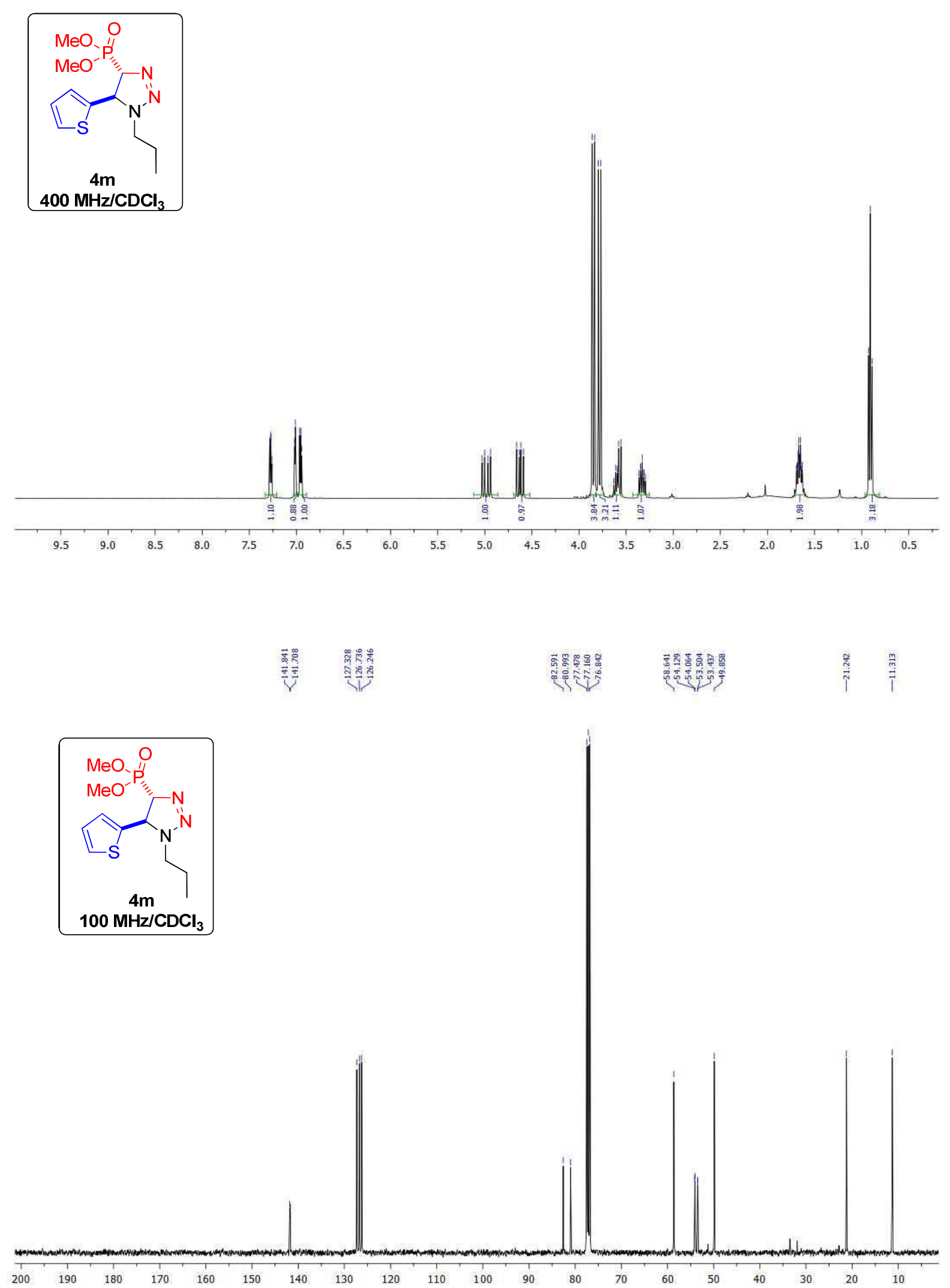

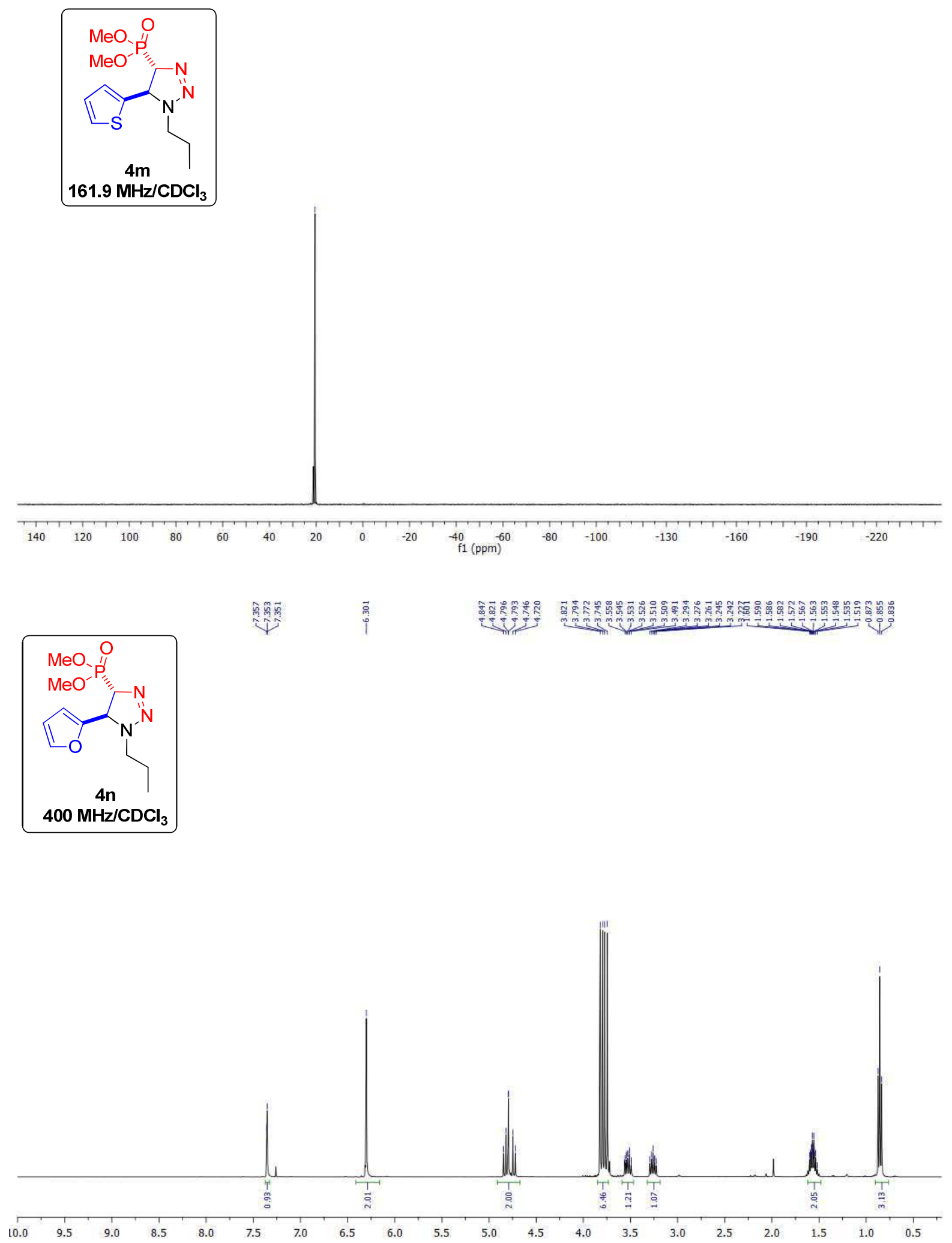


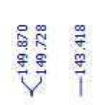

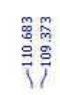

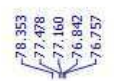

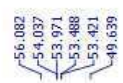

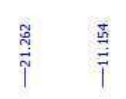
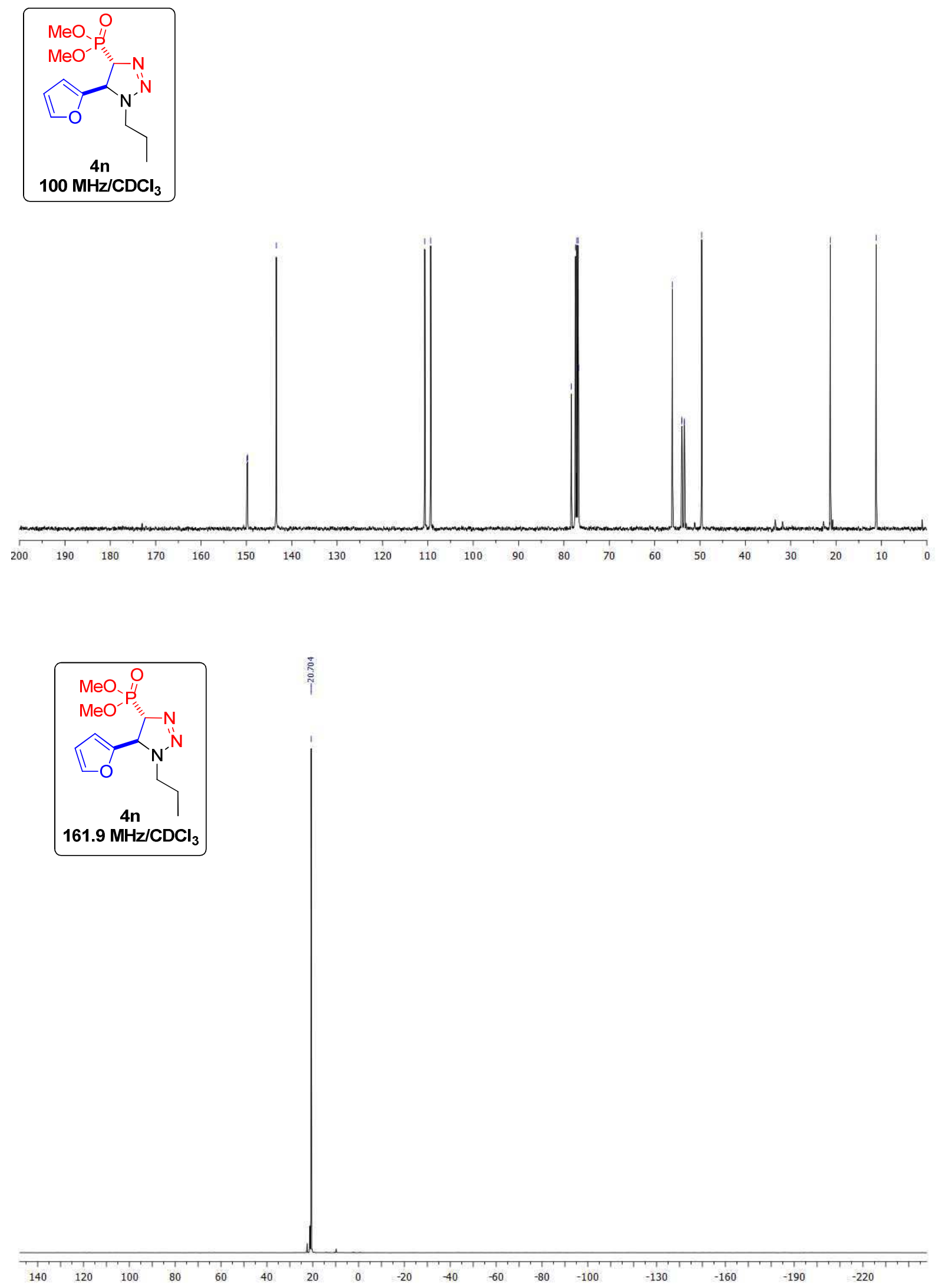

46 

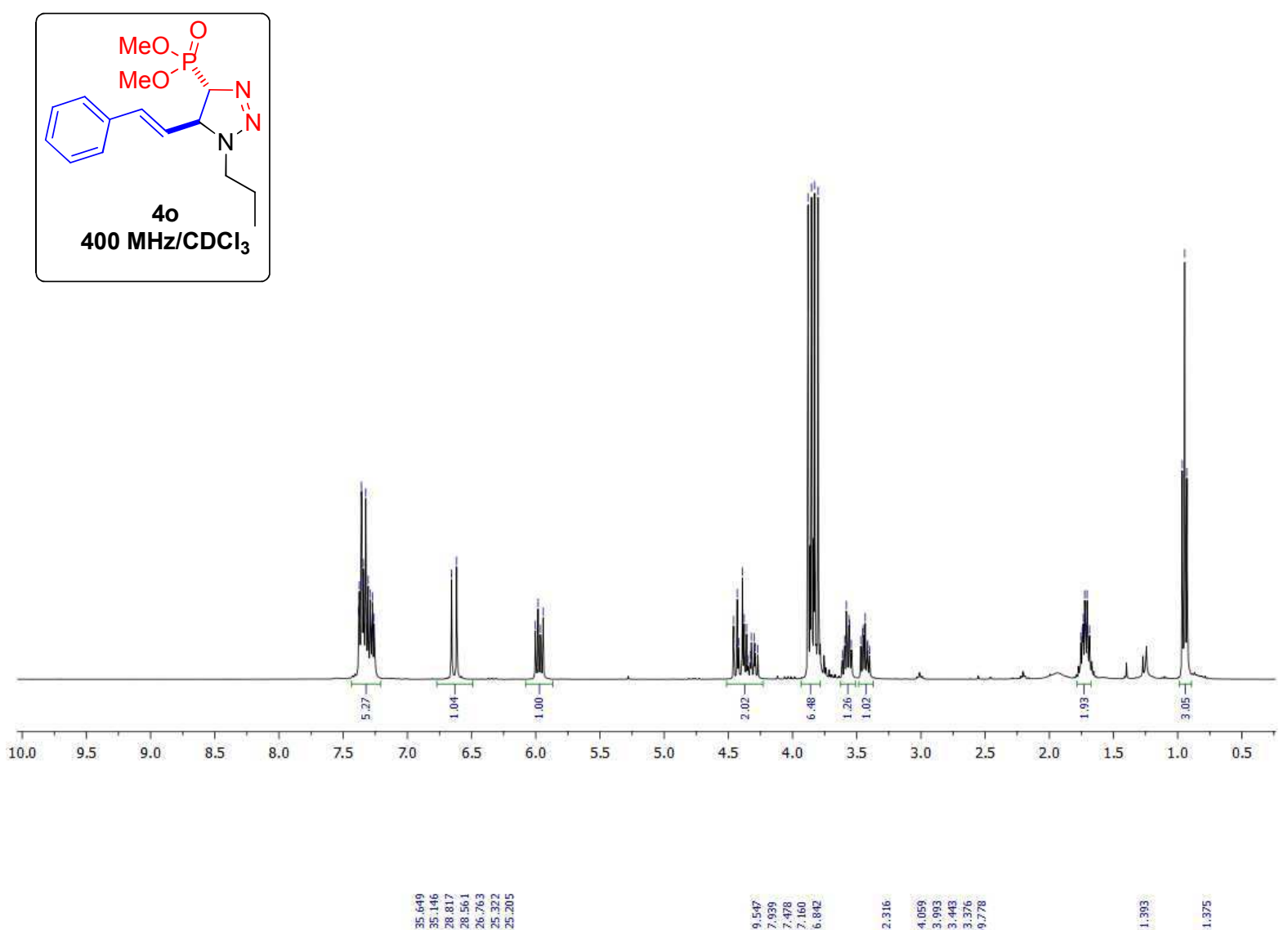

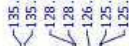

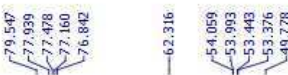

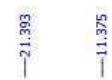
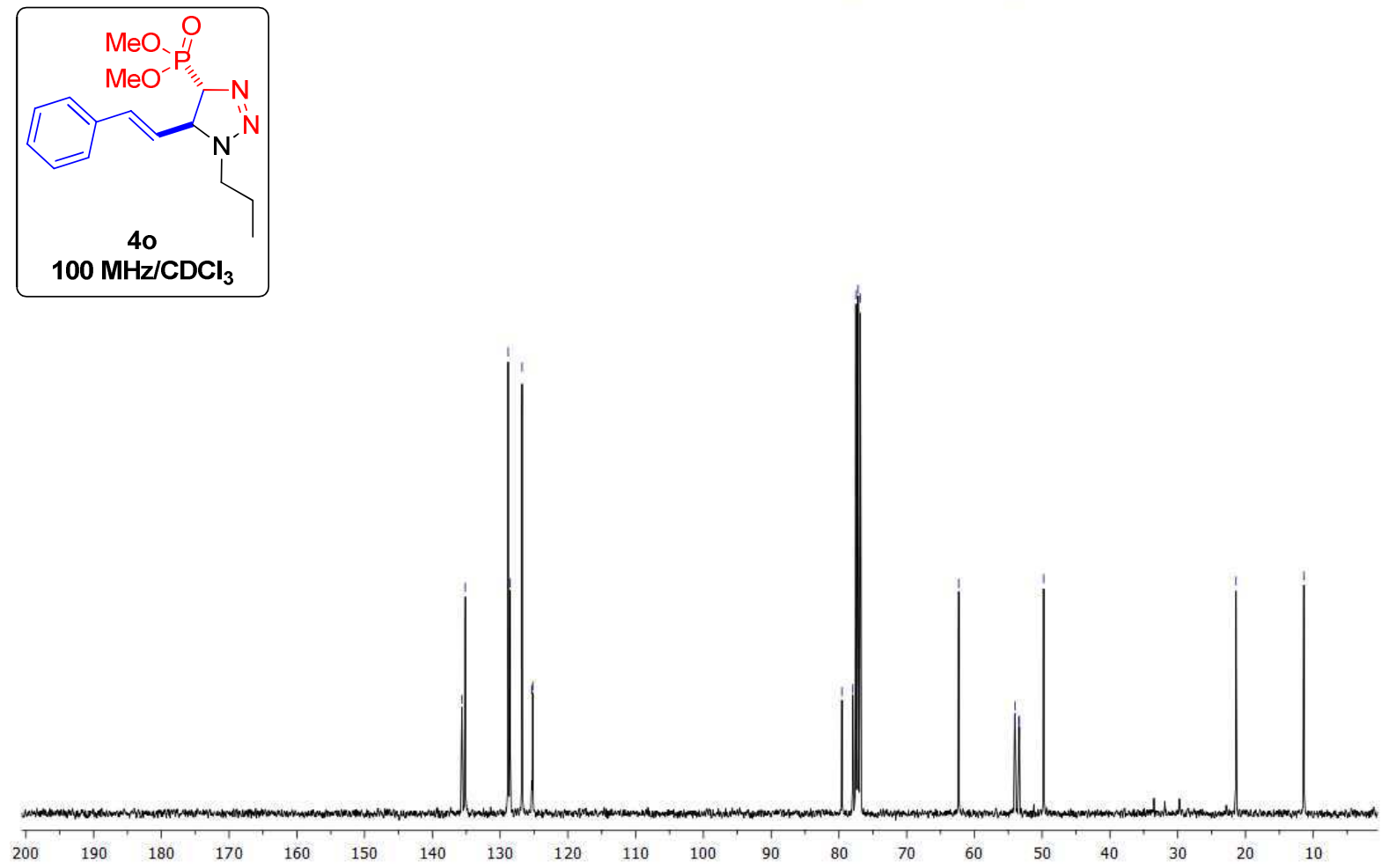

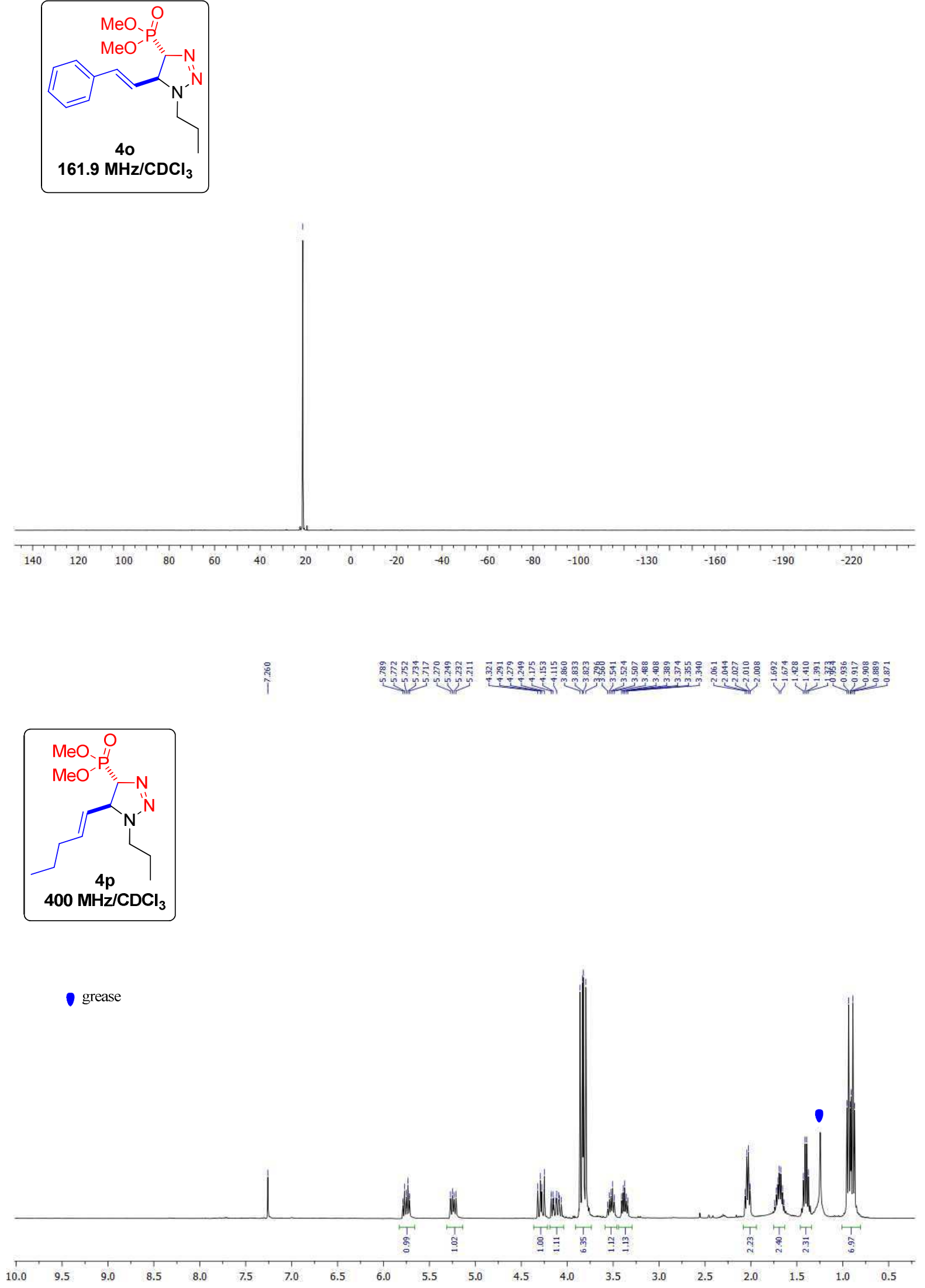

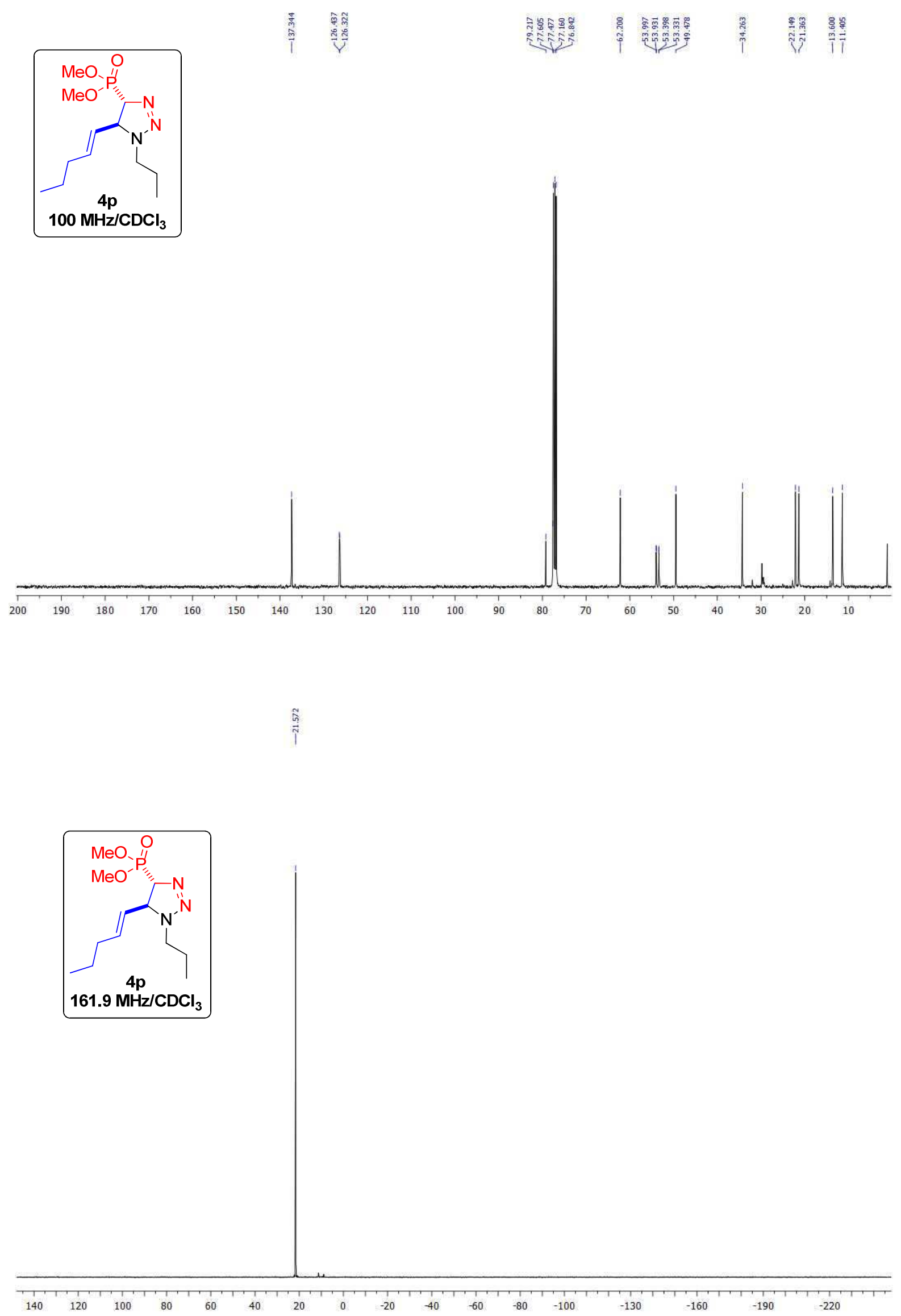


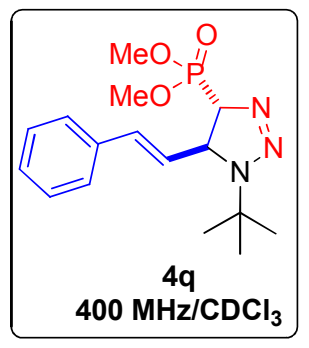

$400 \mathrm{MHz} / \mathrm{CDCl}_{3}$
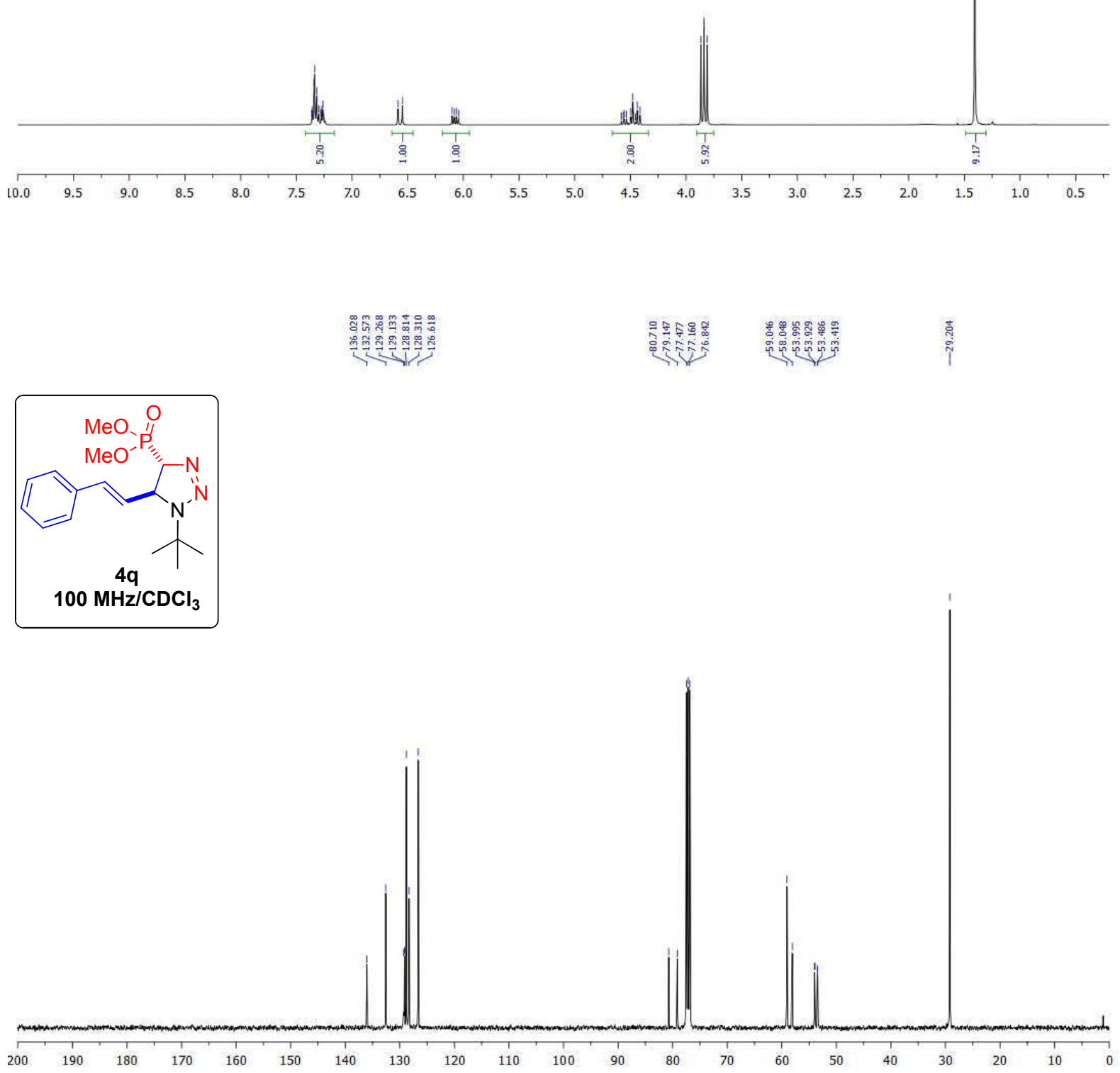


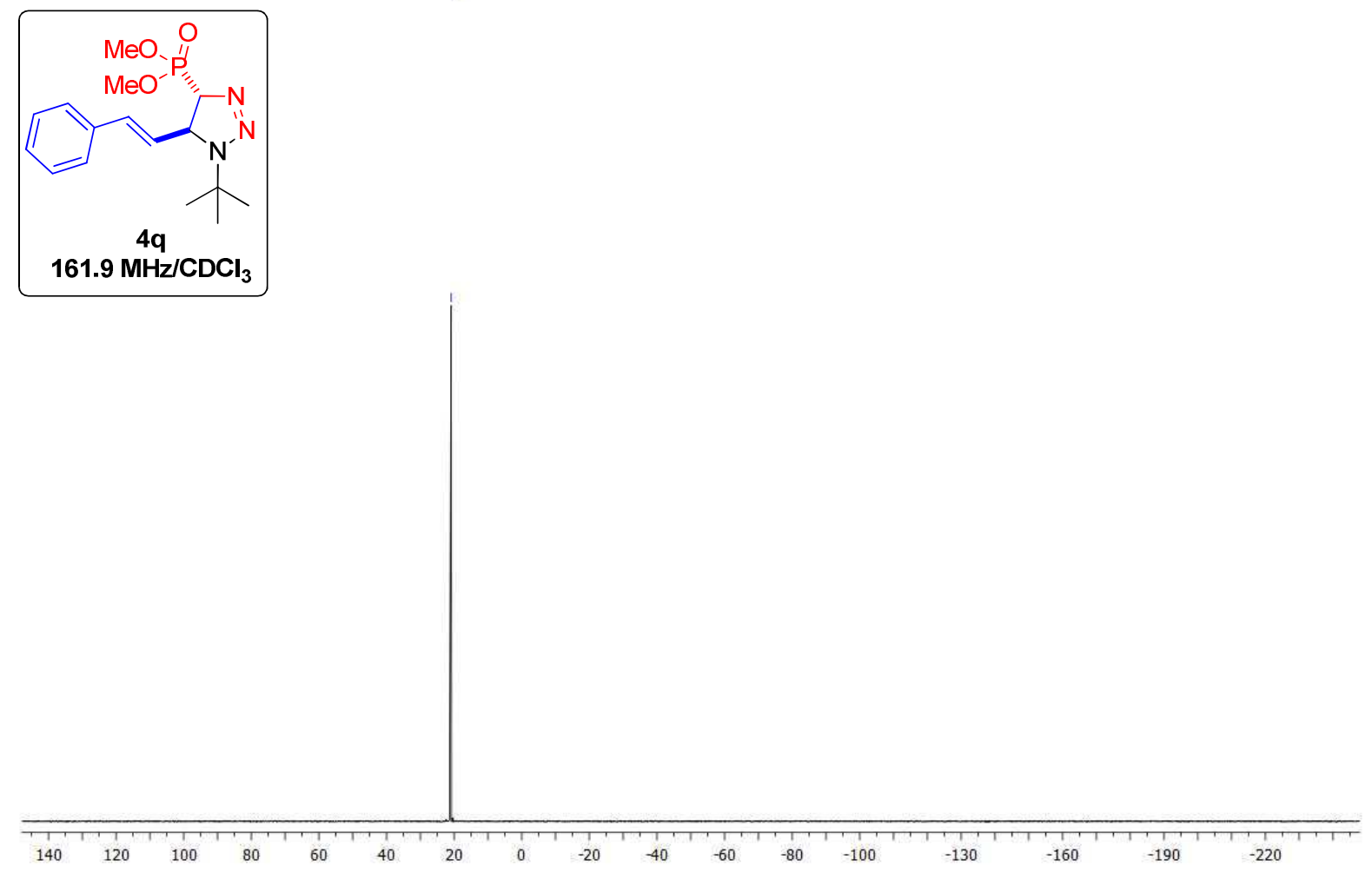

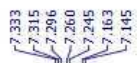

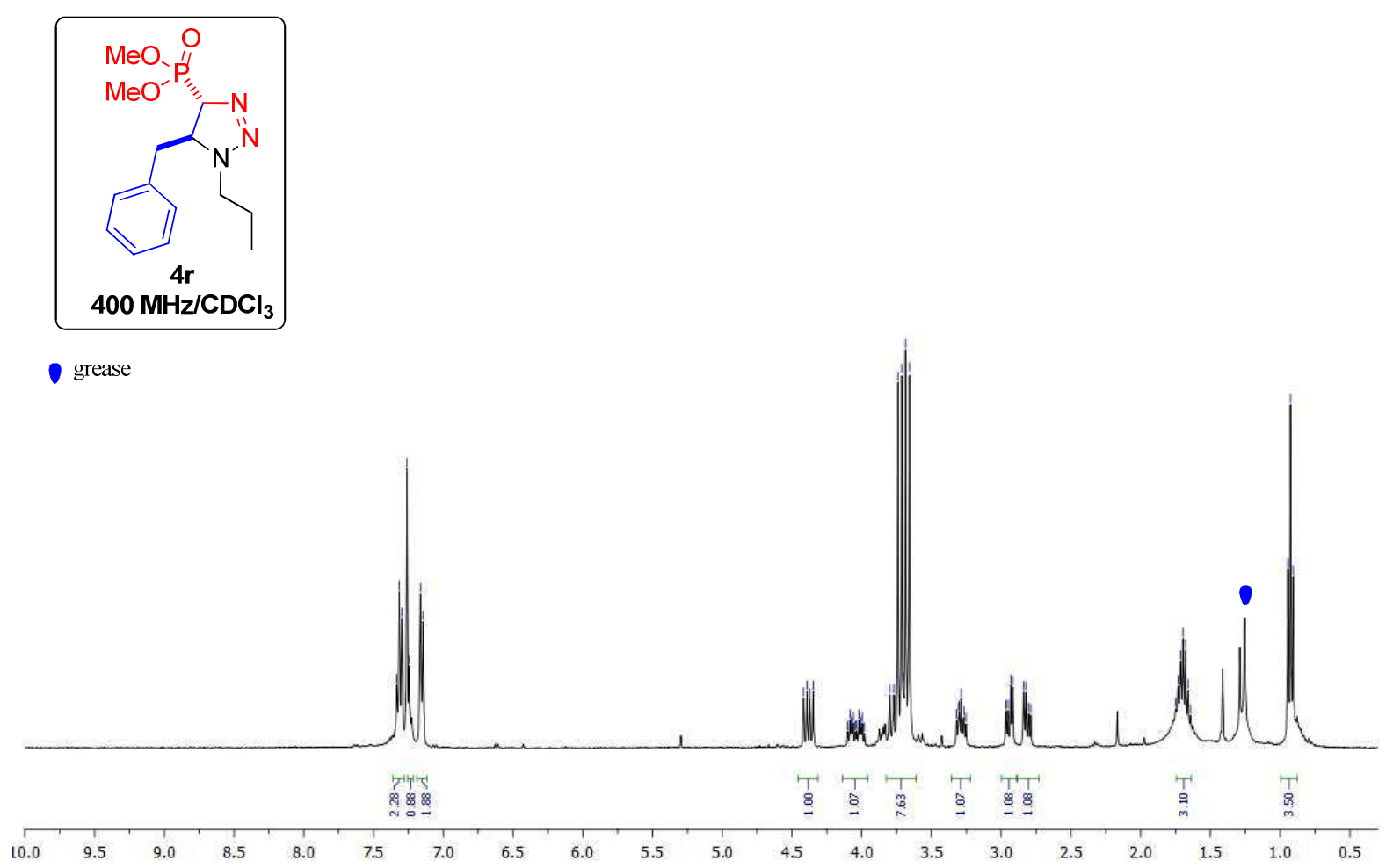



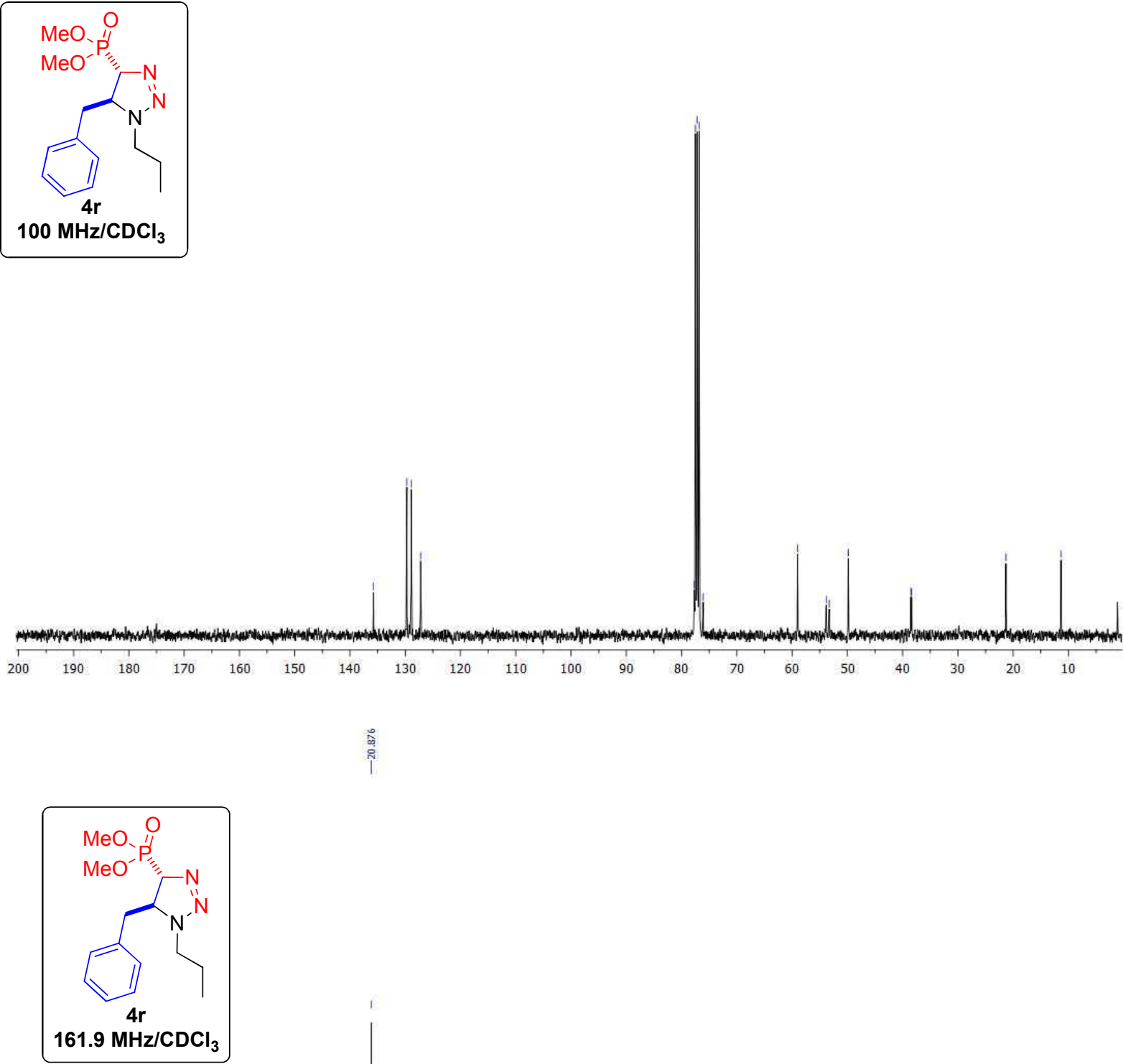

$161.9 \mathrm{MHz} / \mathrm{CDCl}_{3}$ 

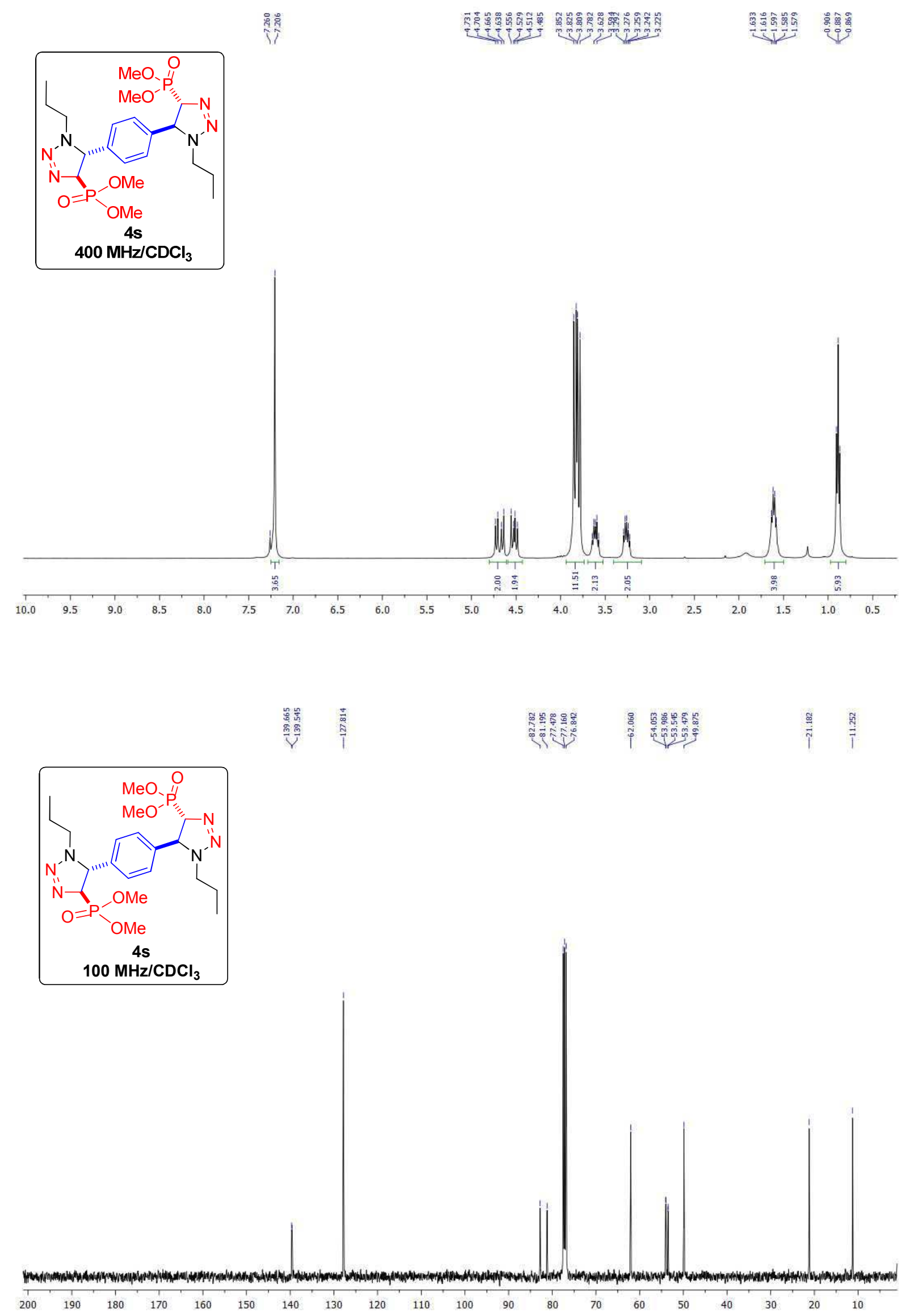

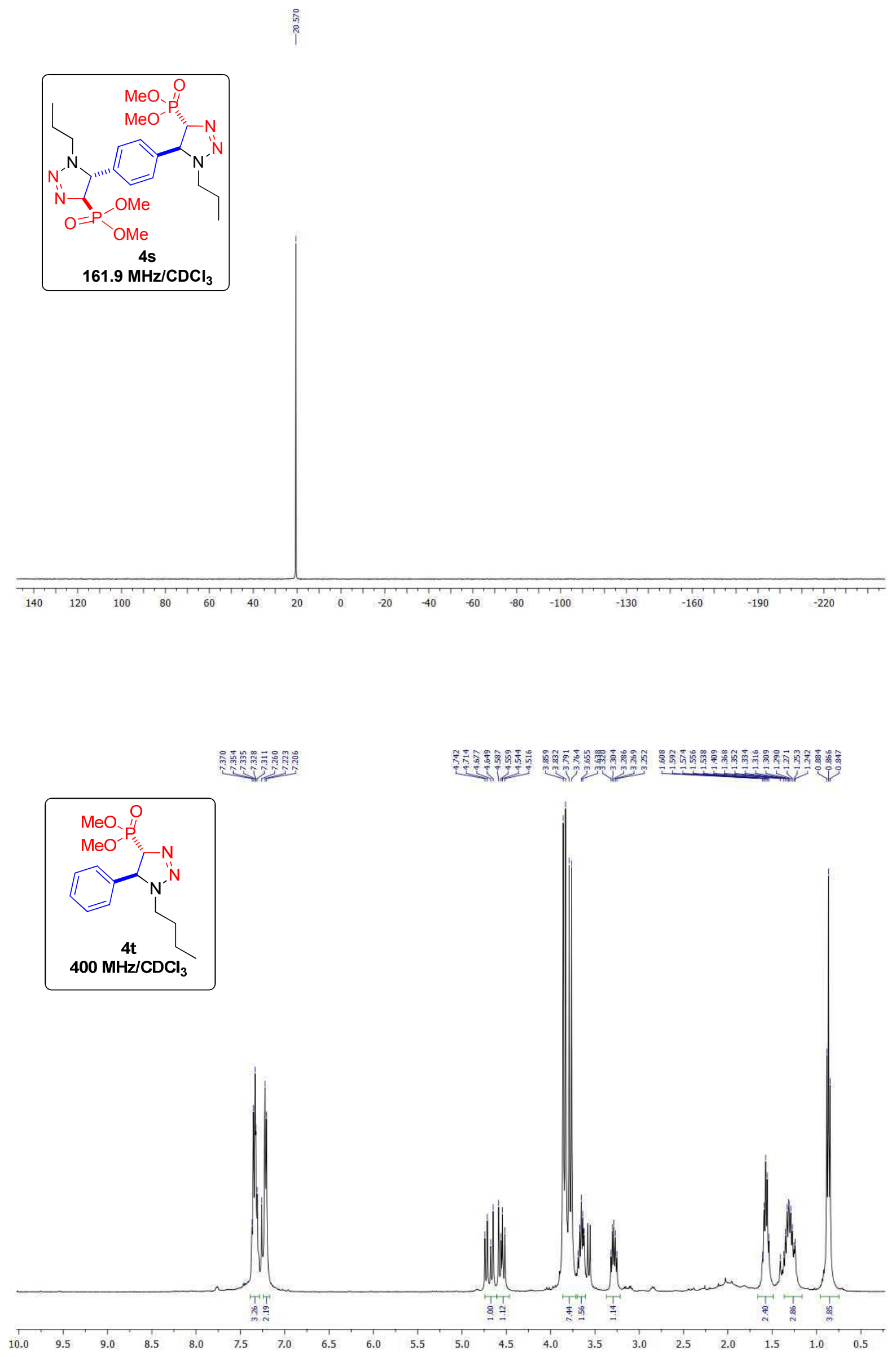


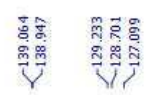

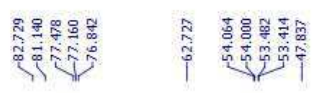

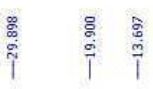
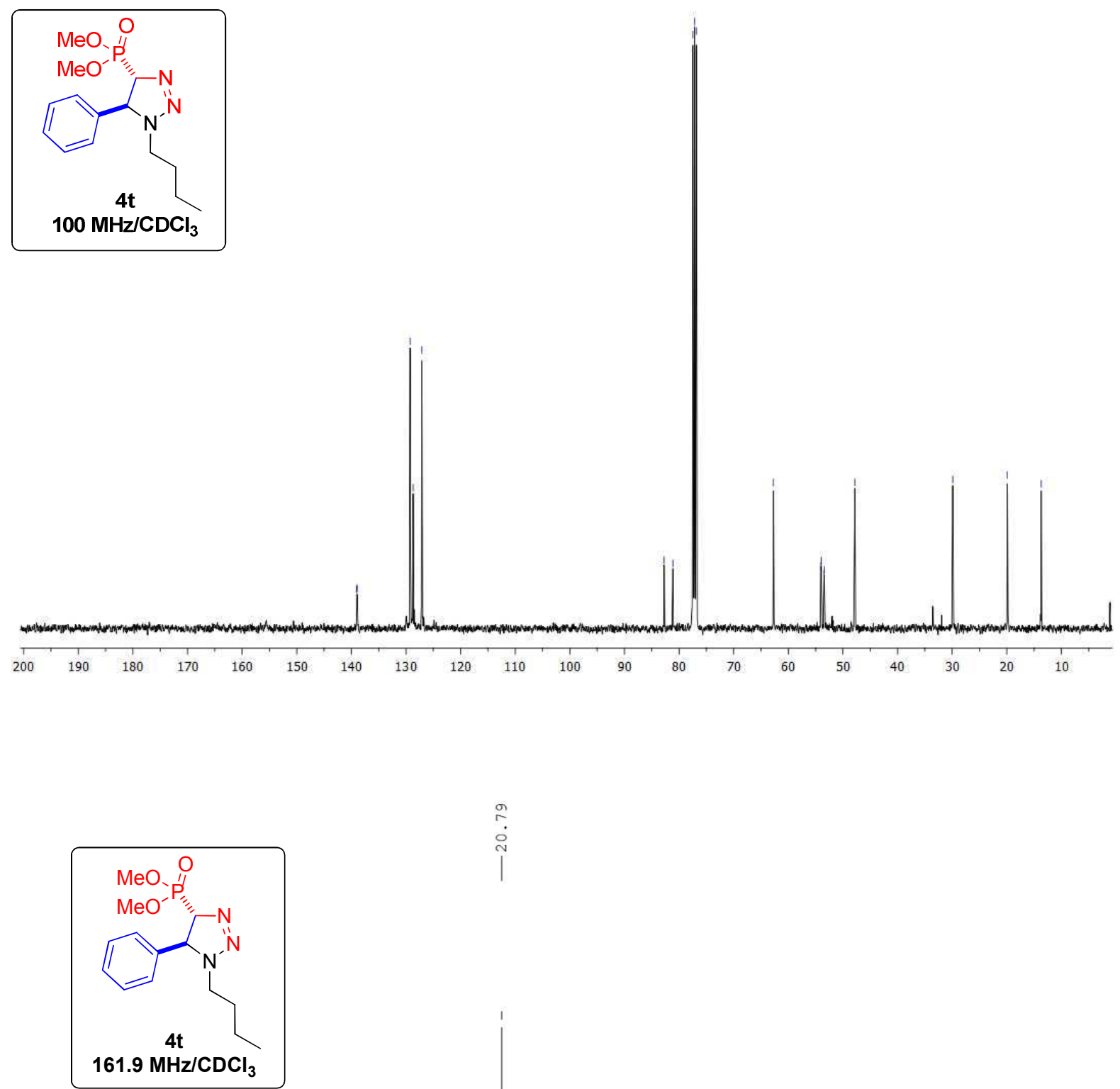

$\stackrel{0}{r}$
$\stackrel{2}{1}$

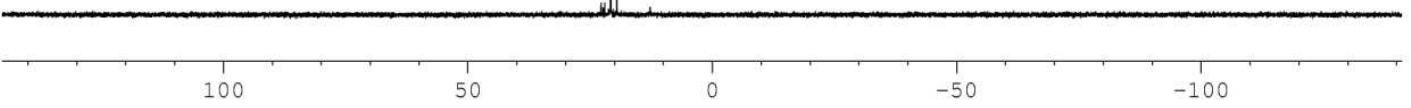

55 

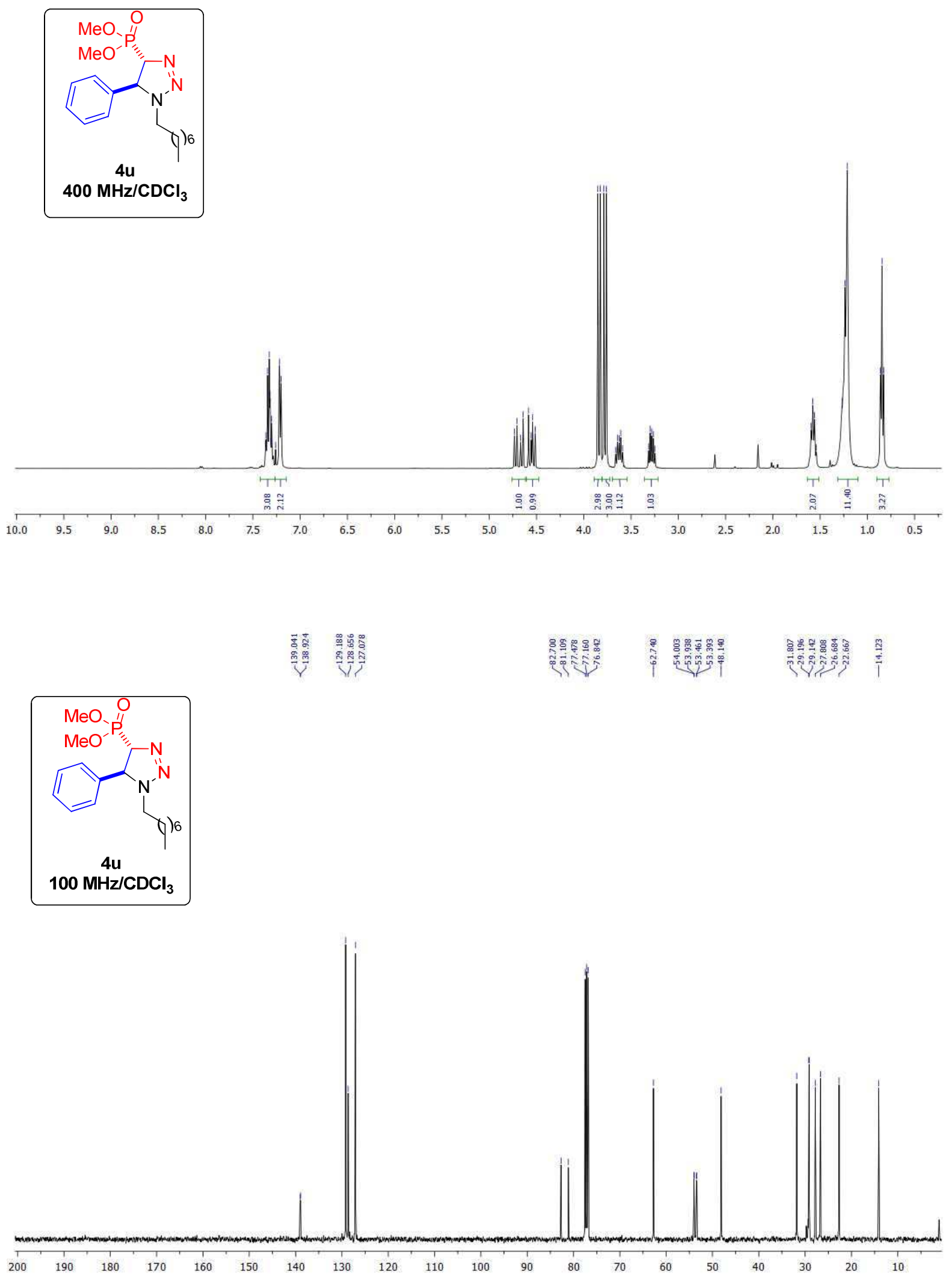


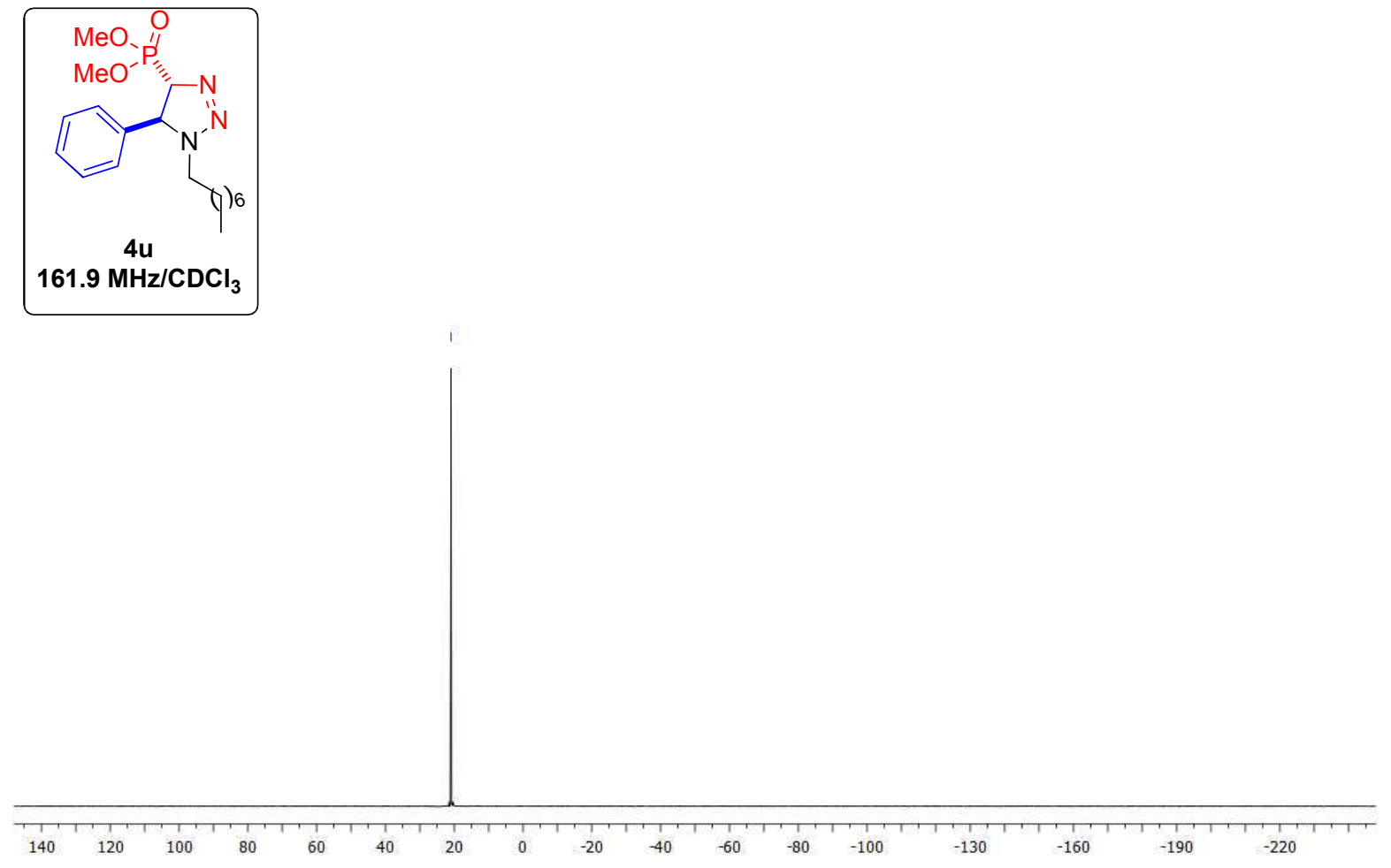

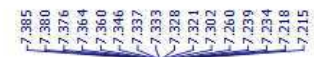

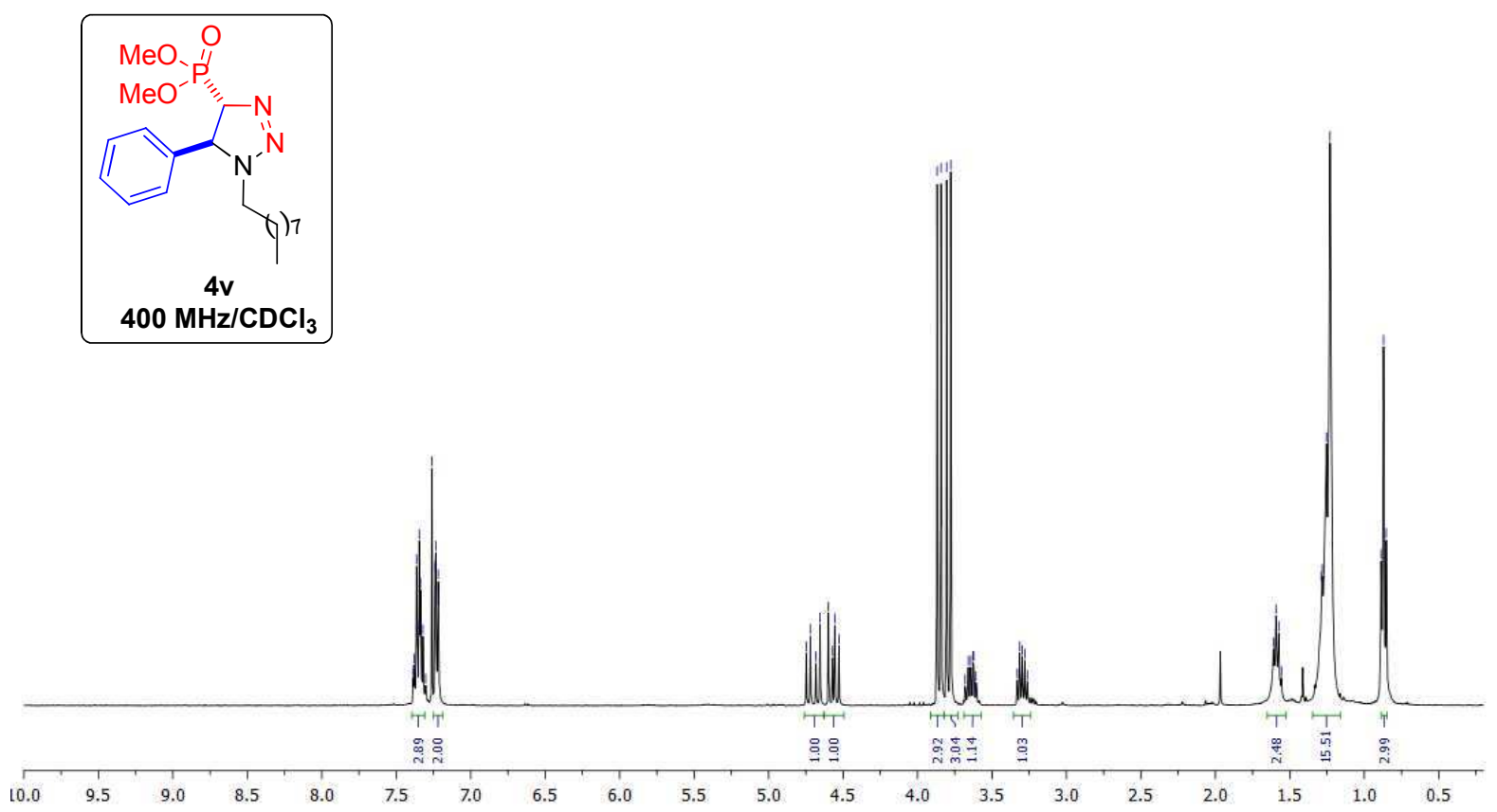



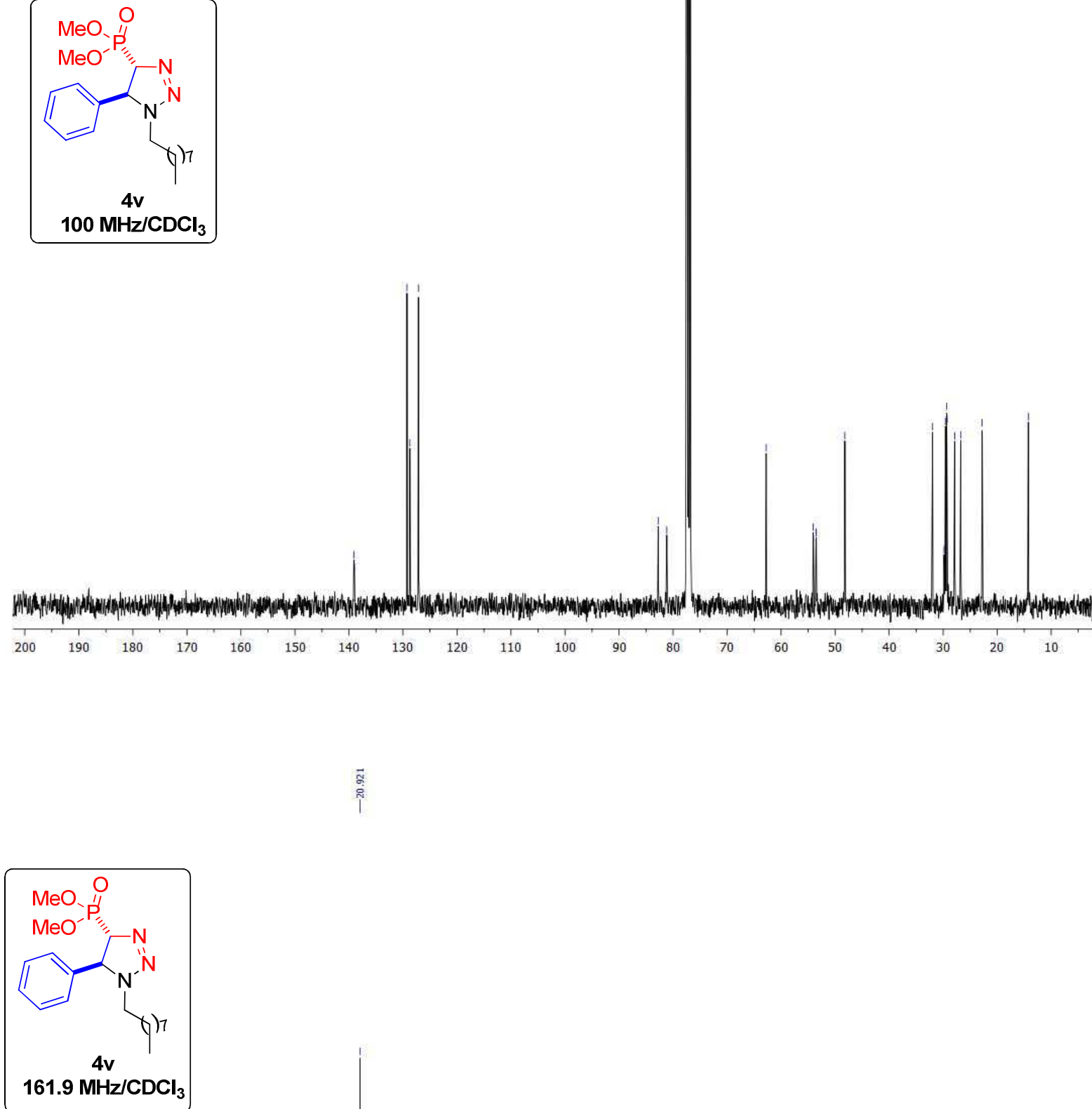

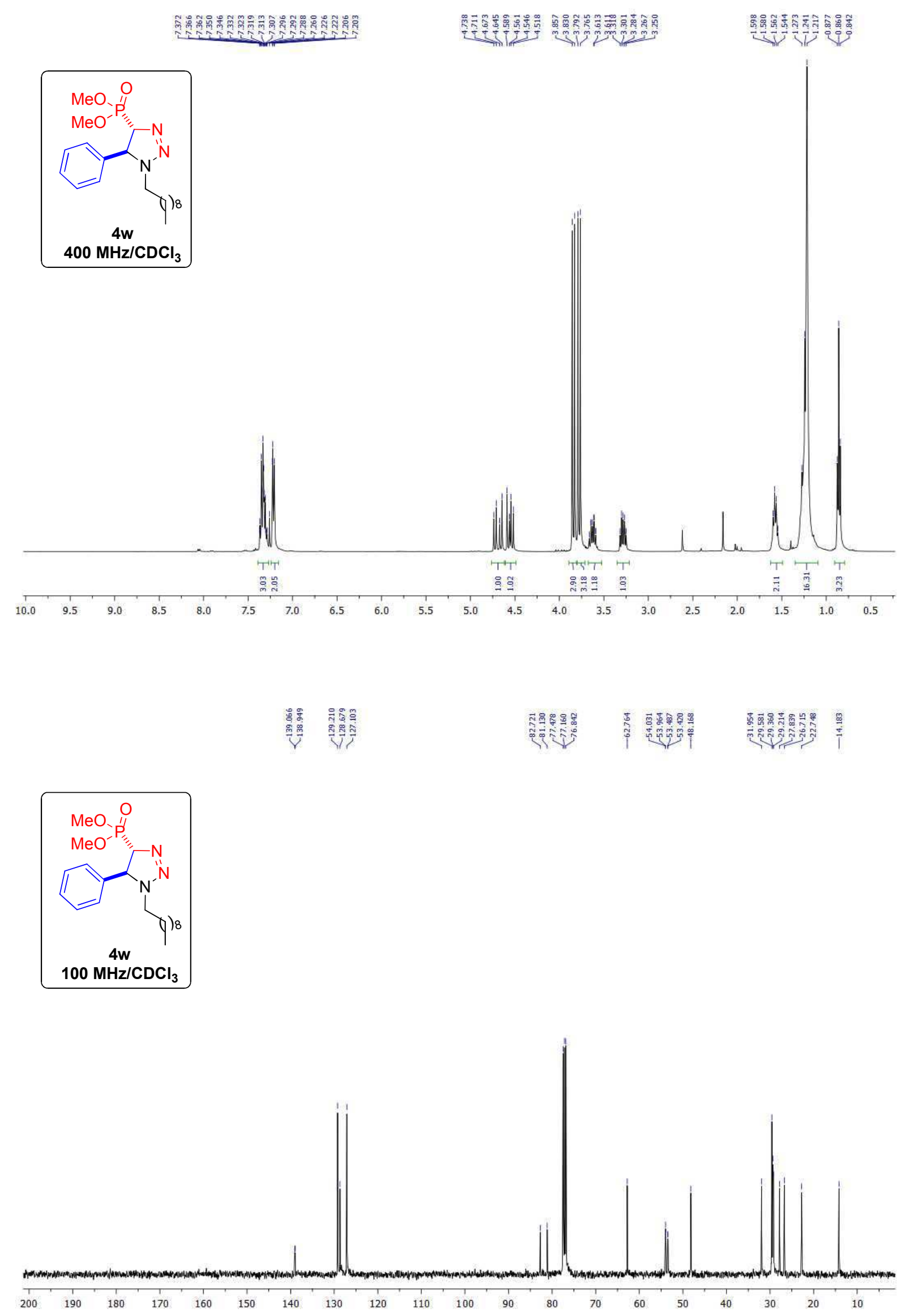

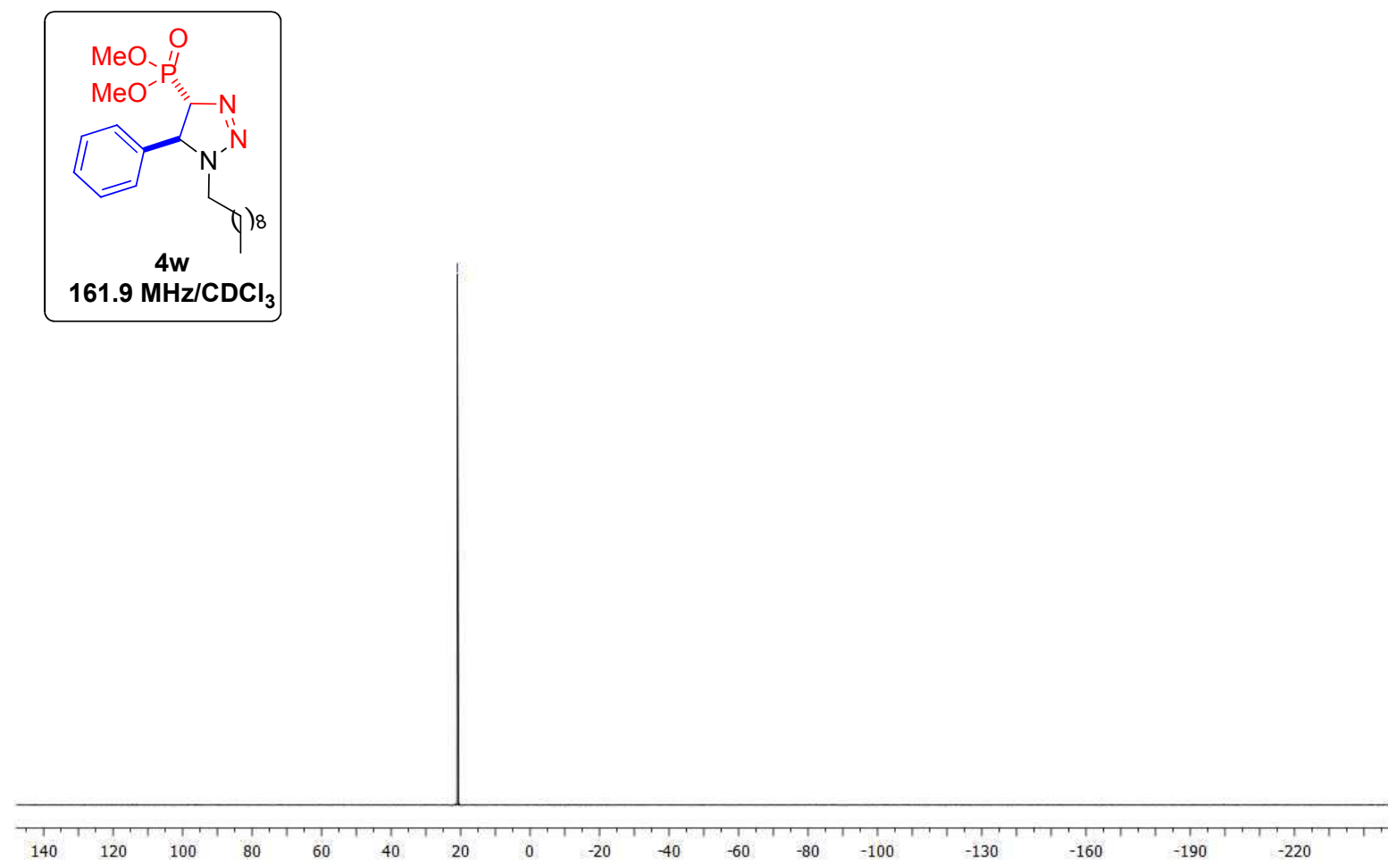

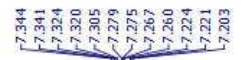

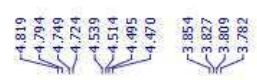
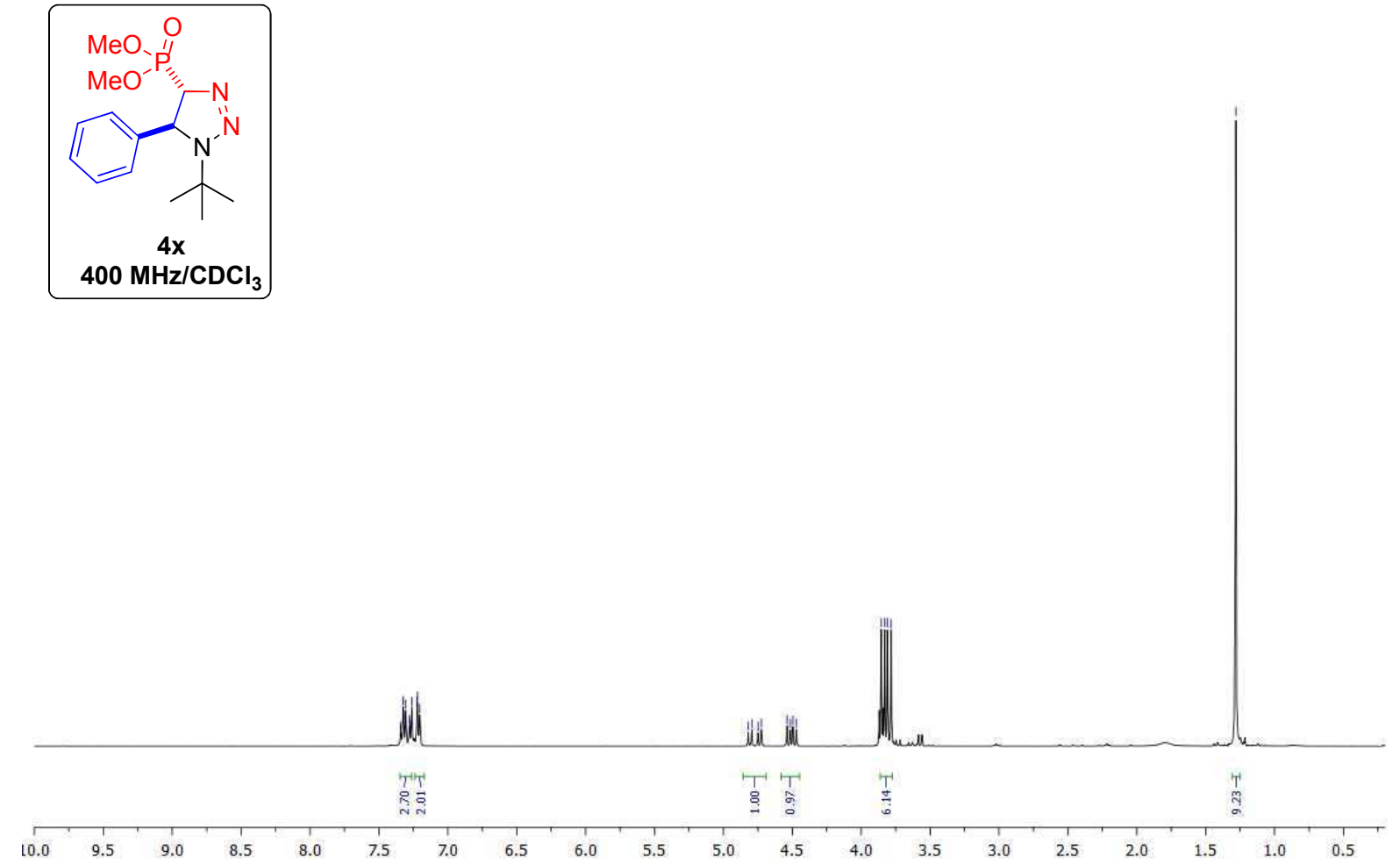


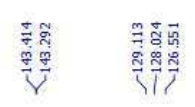

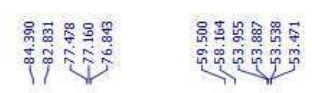

iุ

$\stackrel{0}{i}$
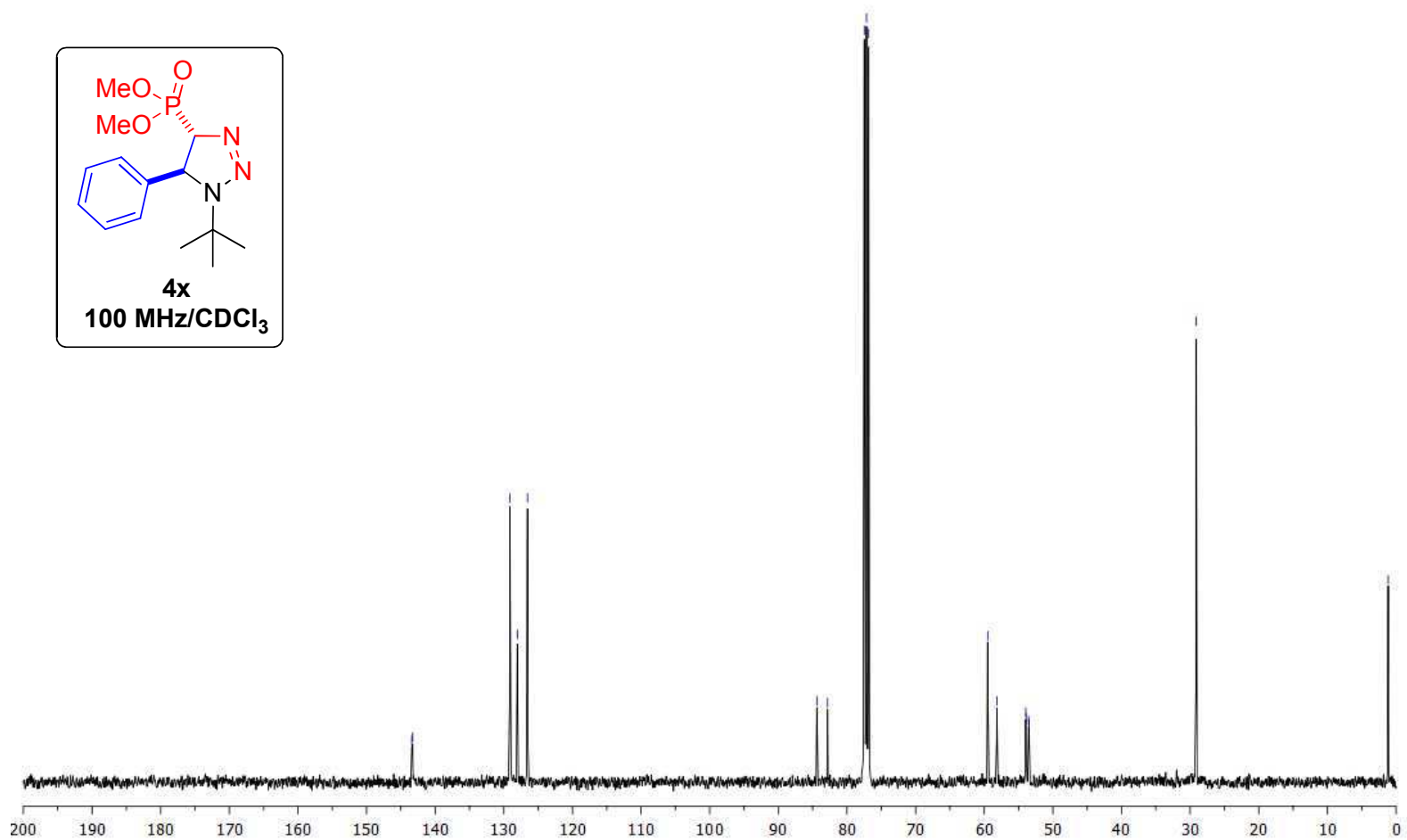

i⿱

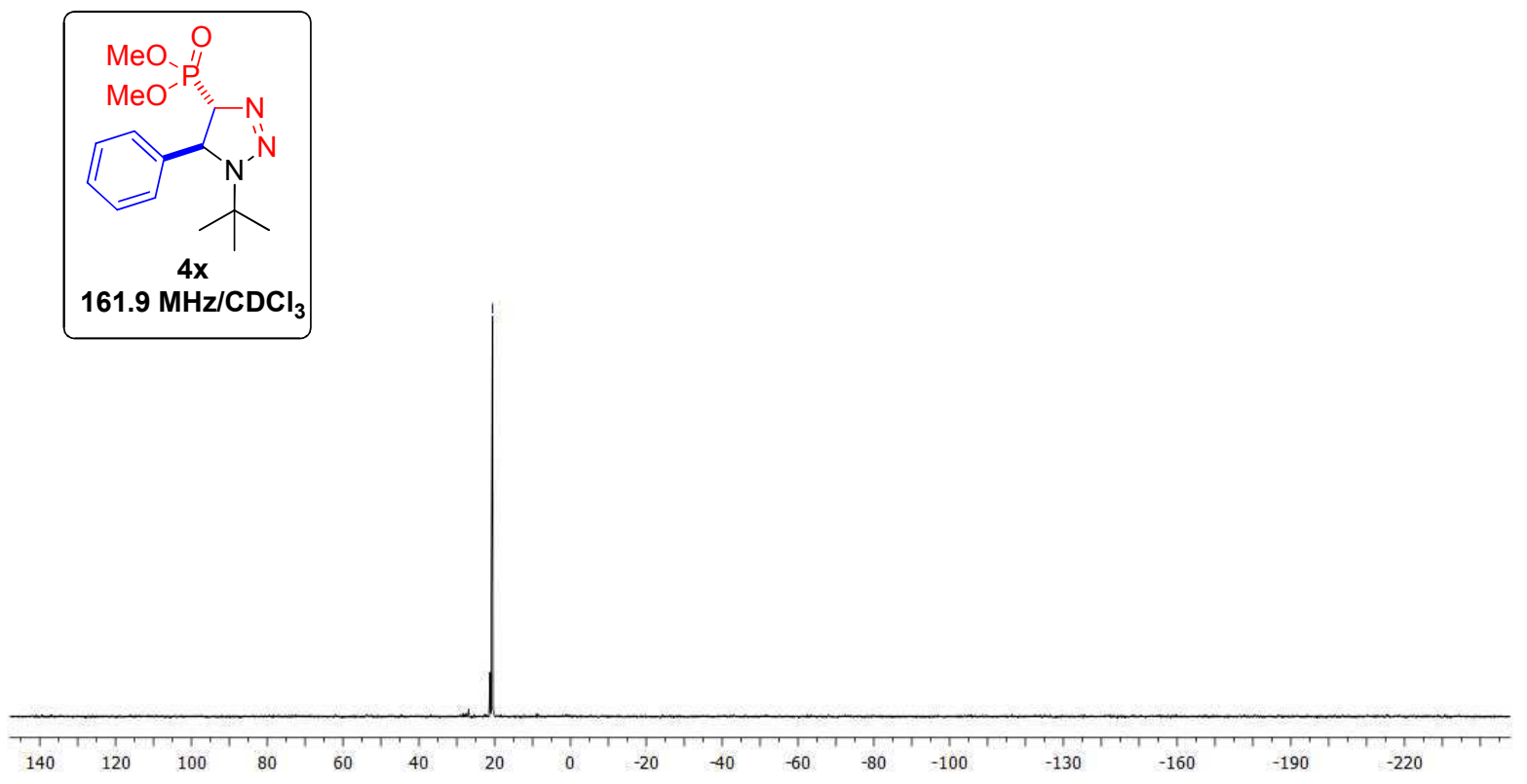

61 


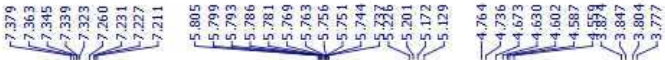
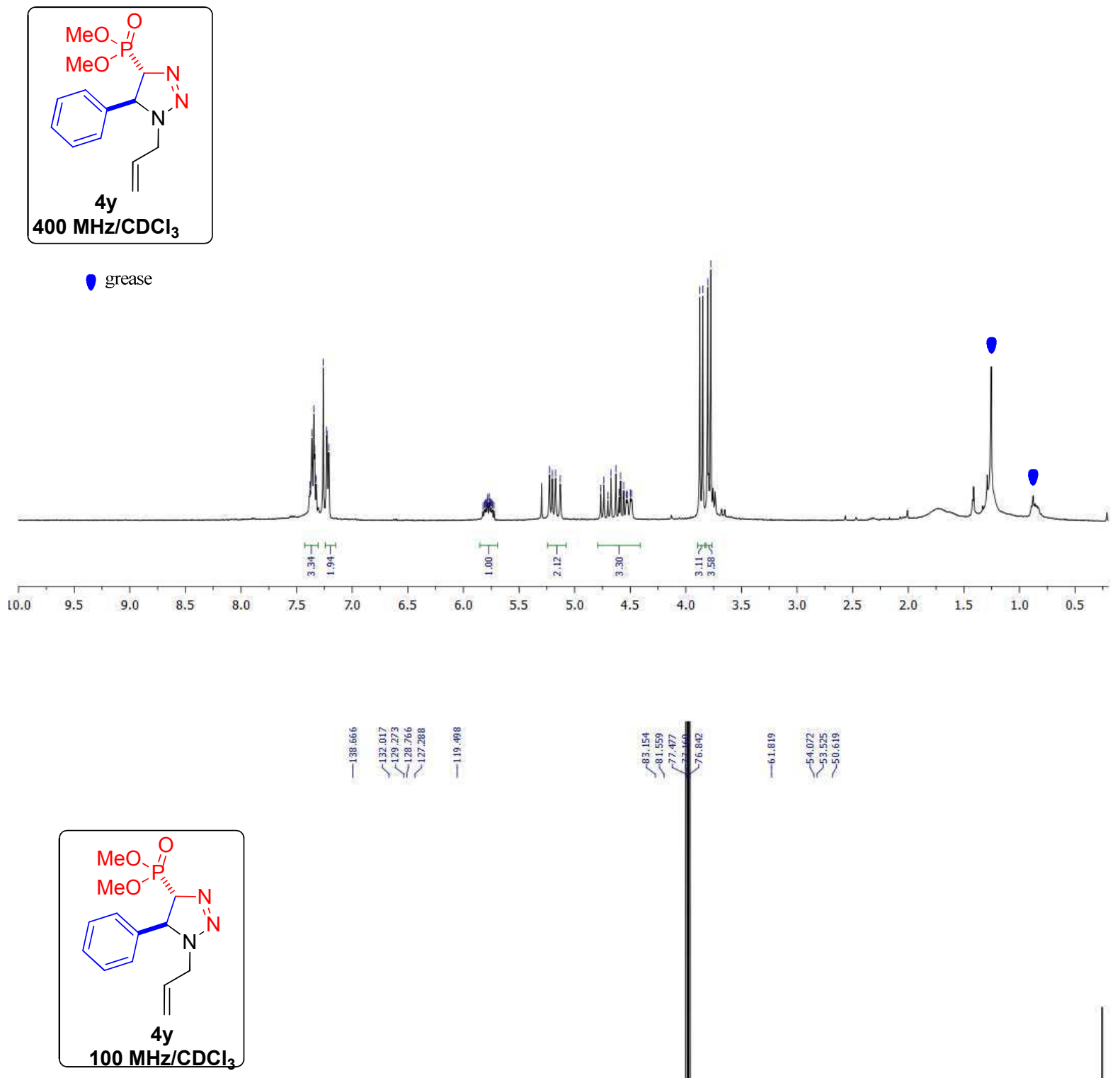

grease

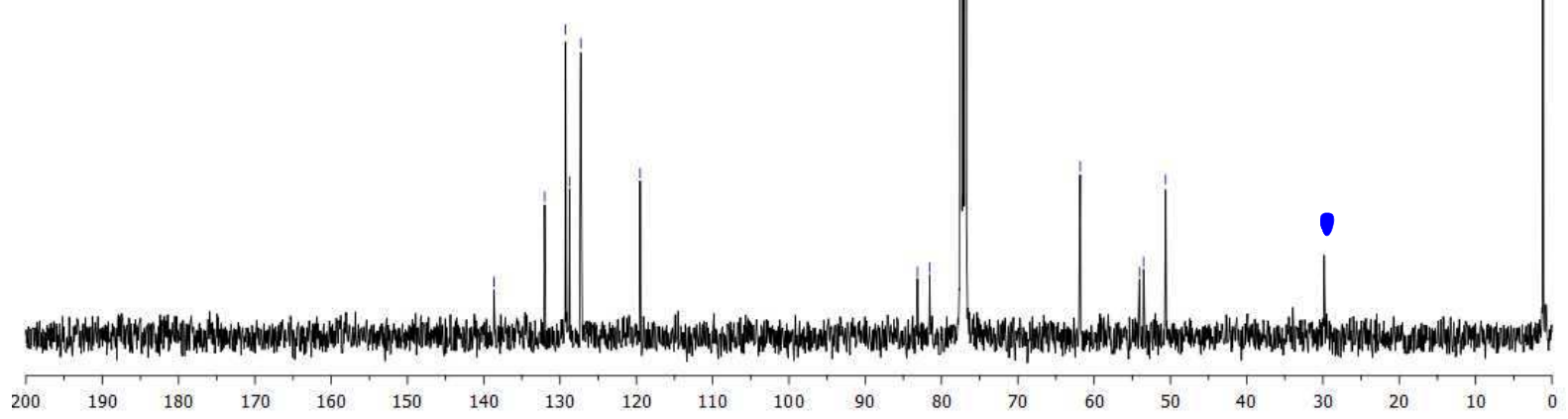



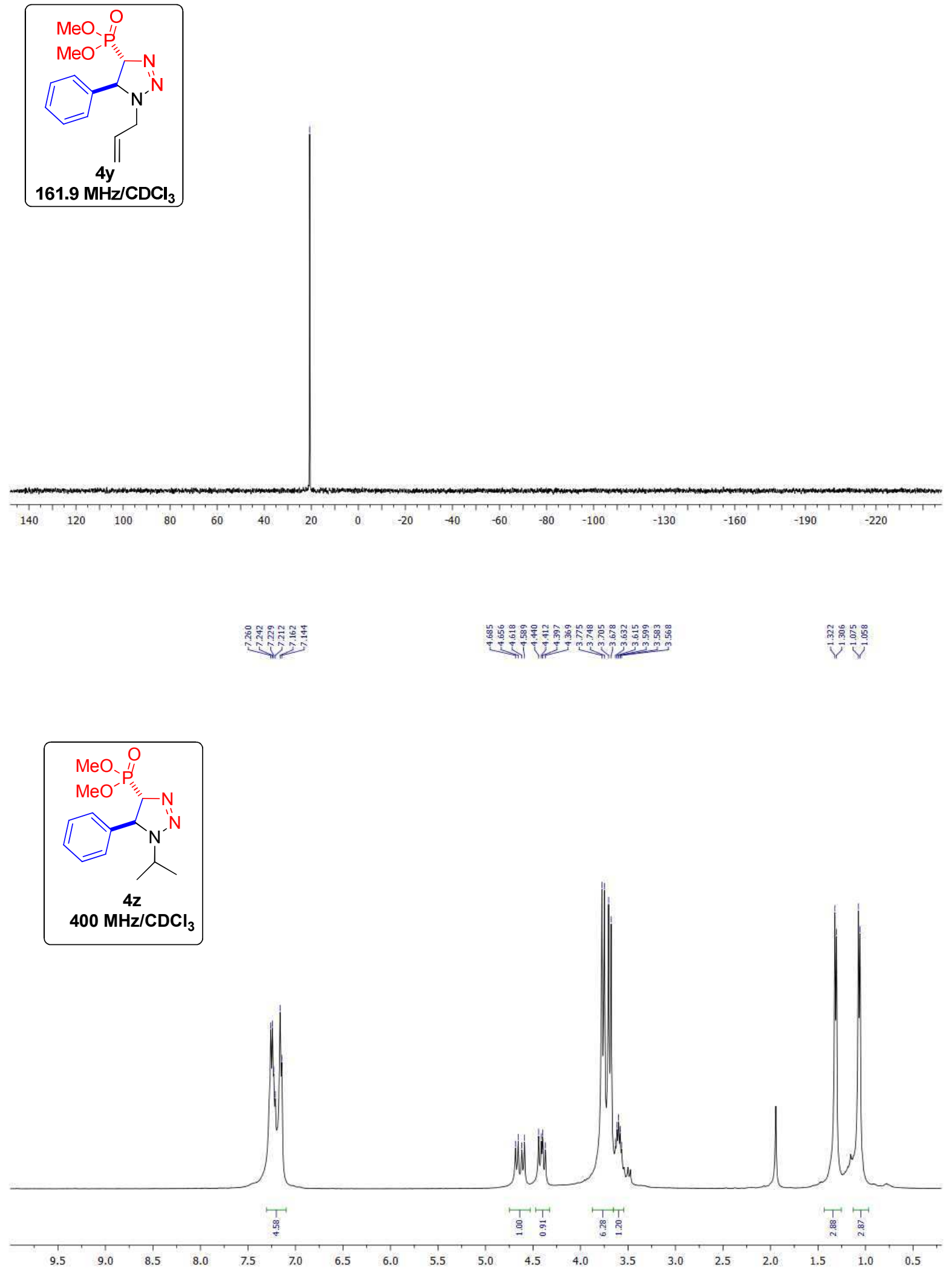


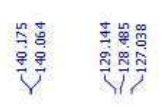

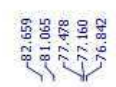

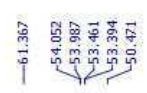

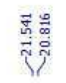

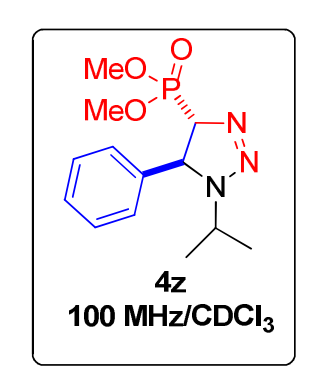

I
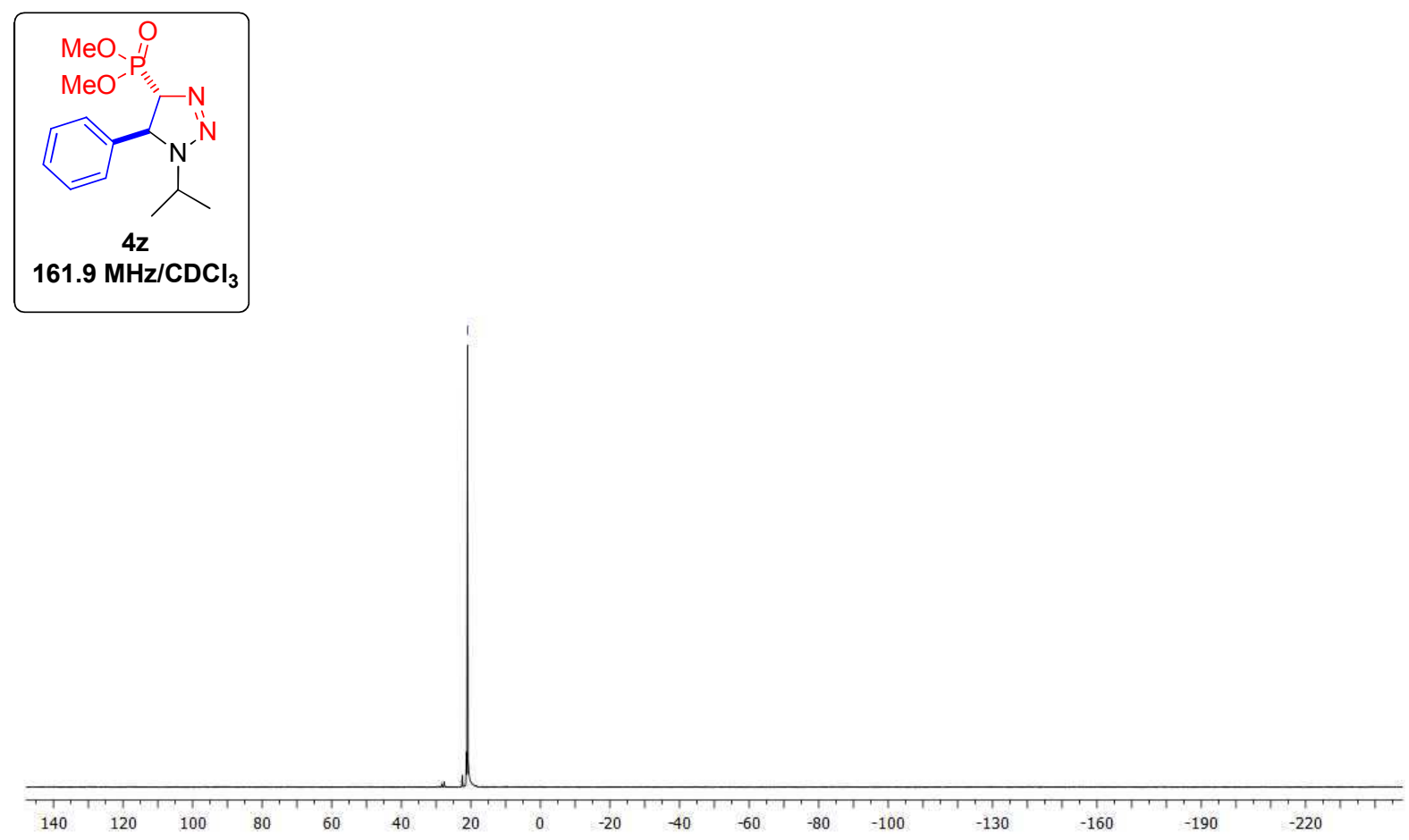

64 


$400 \mathrm{MHz} / \mathrm{CDCl}_{3}$

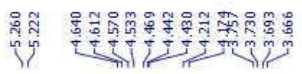
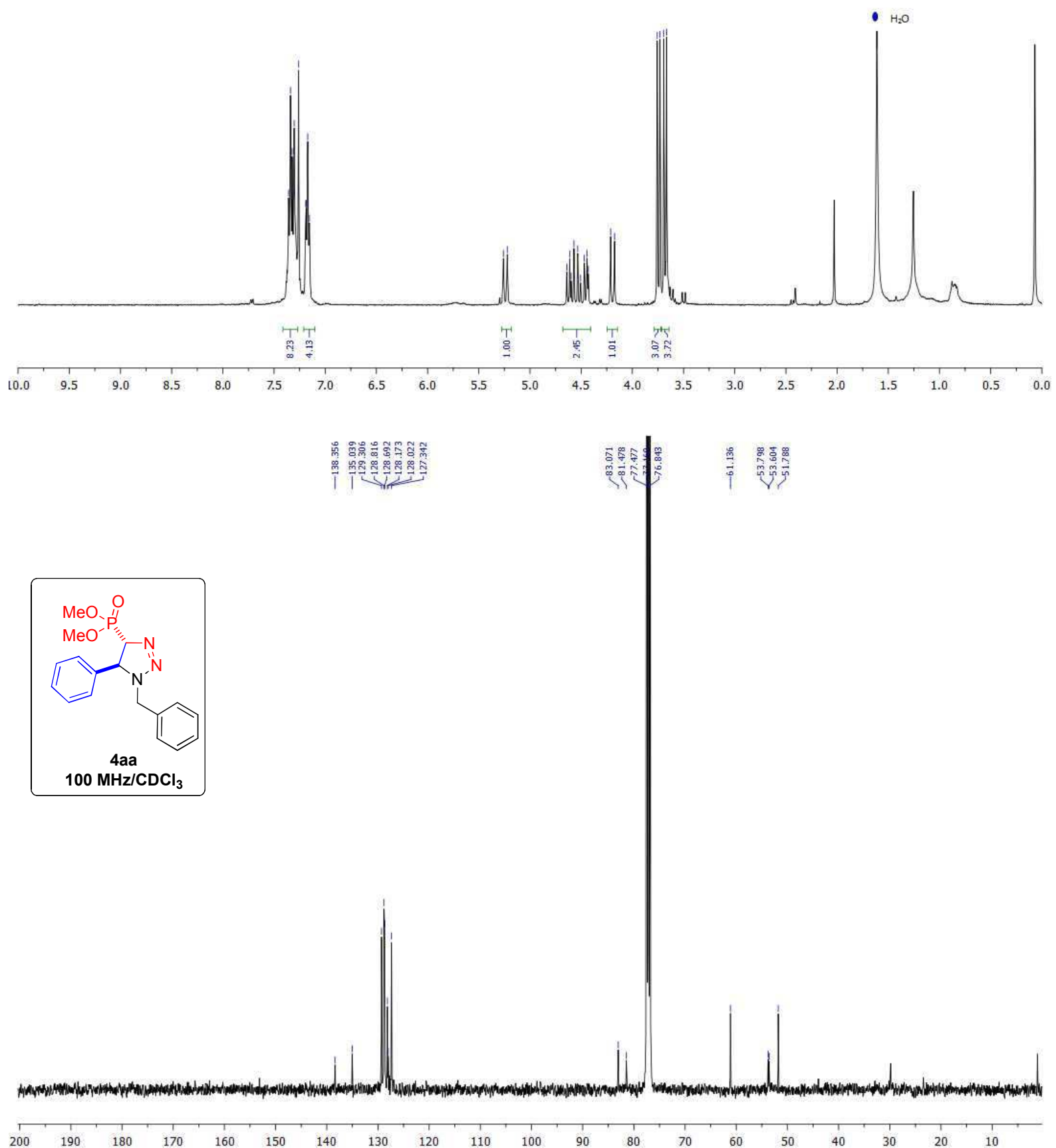


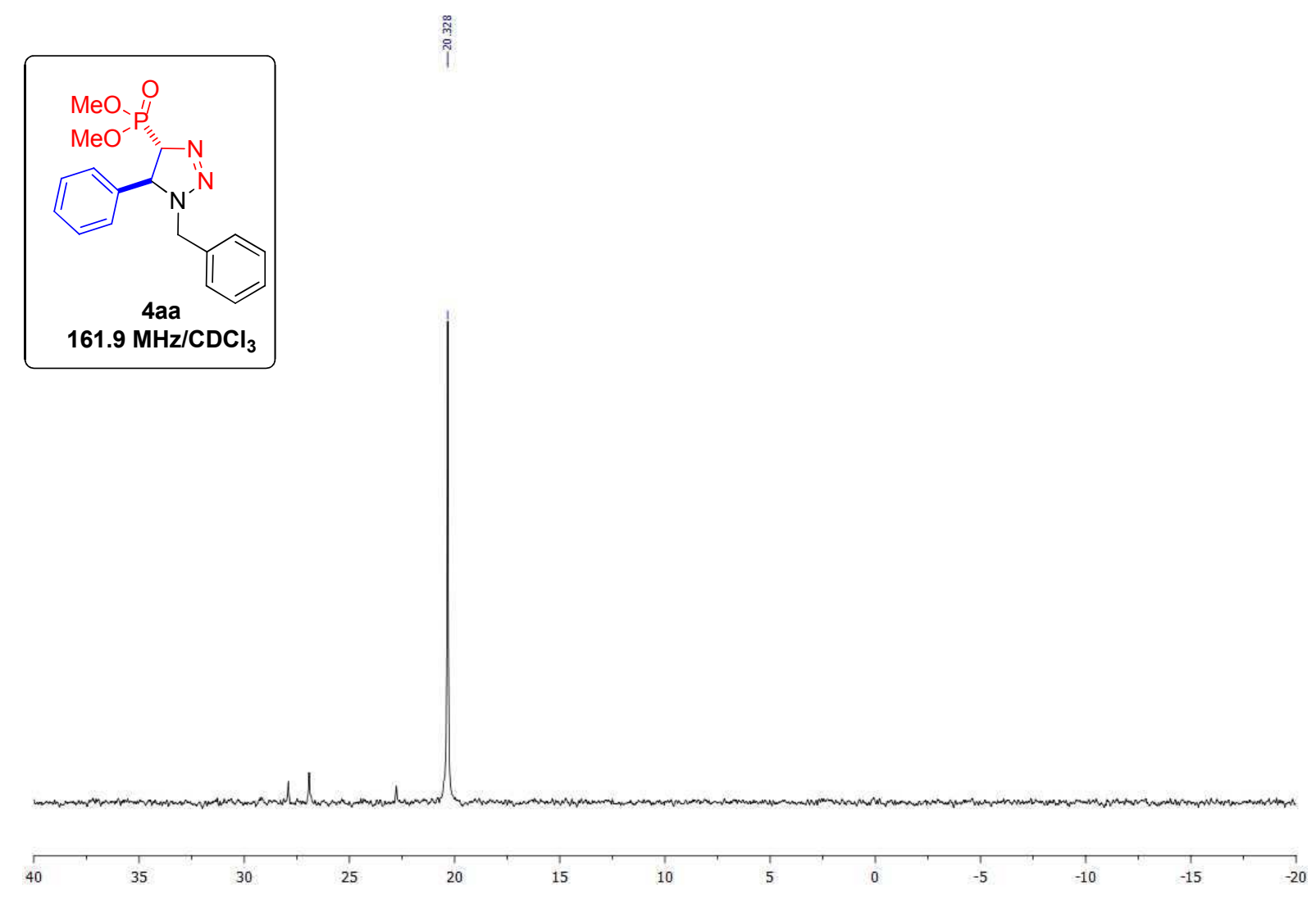

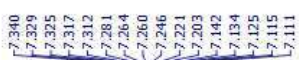

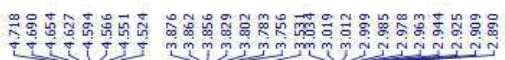
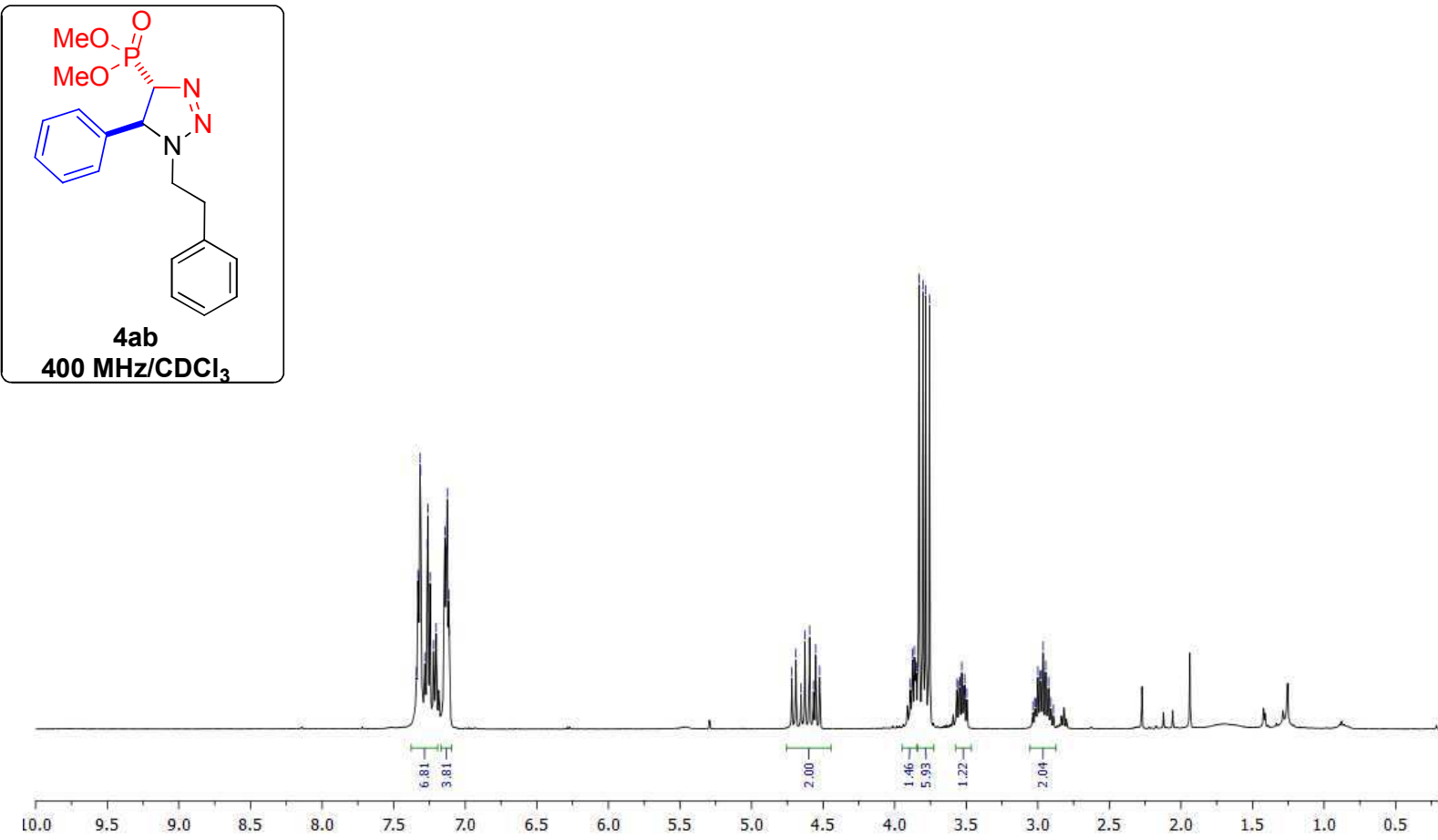


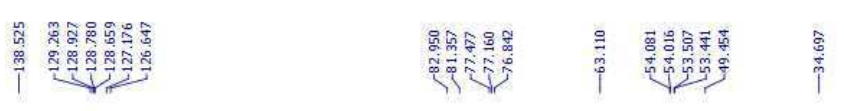
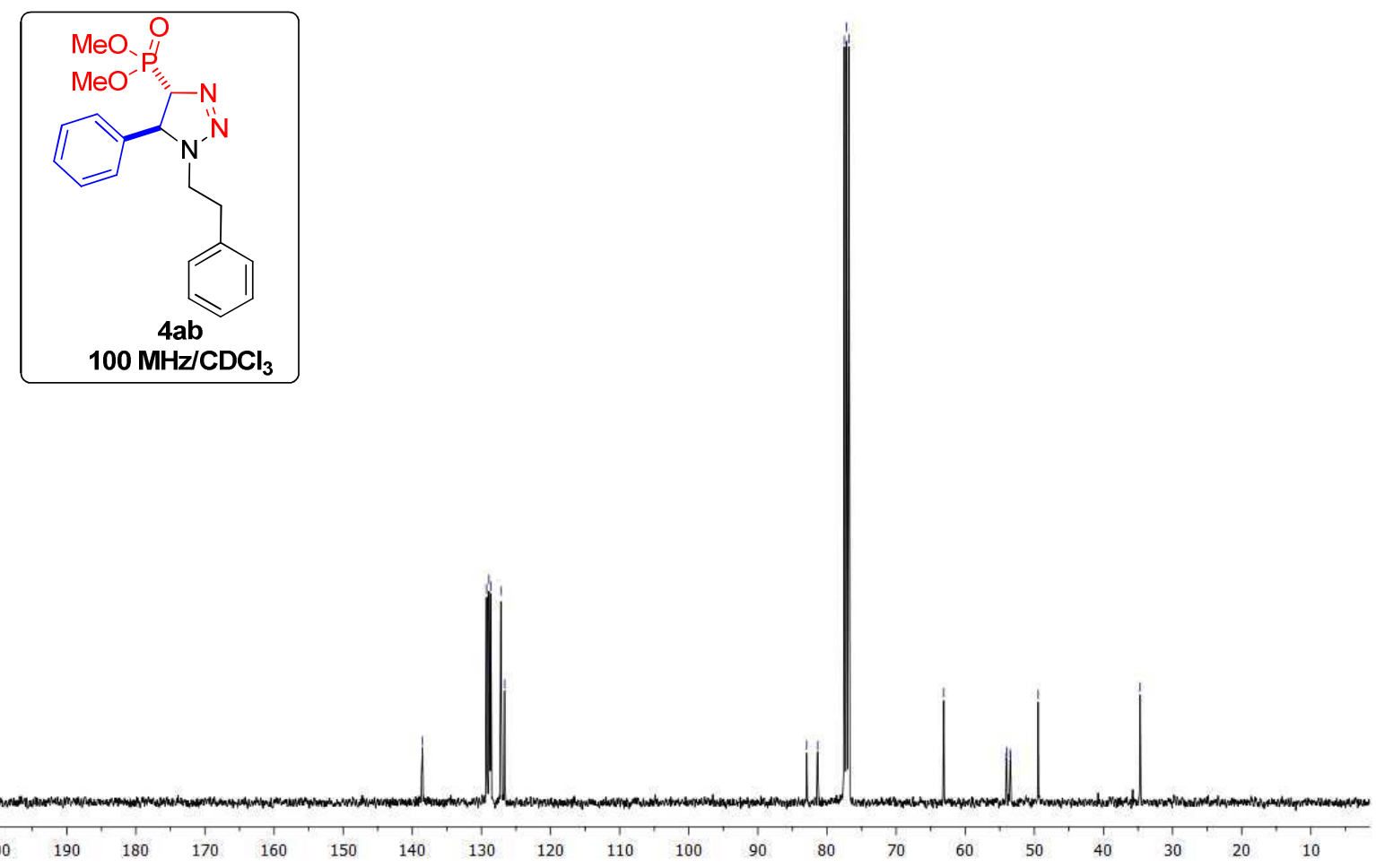

î

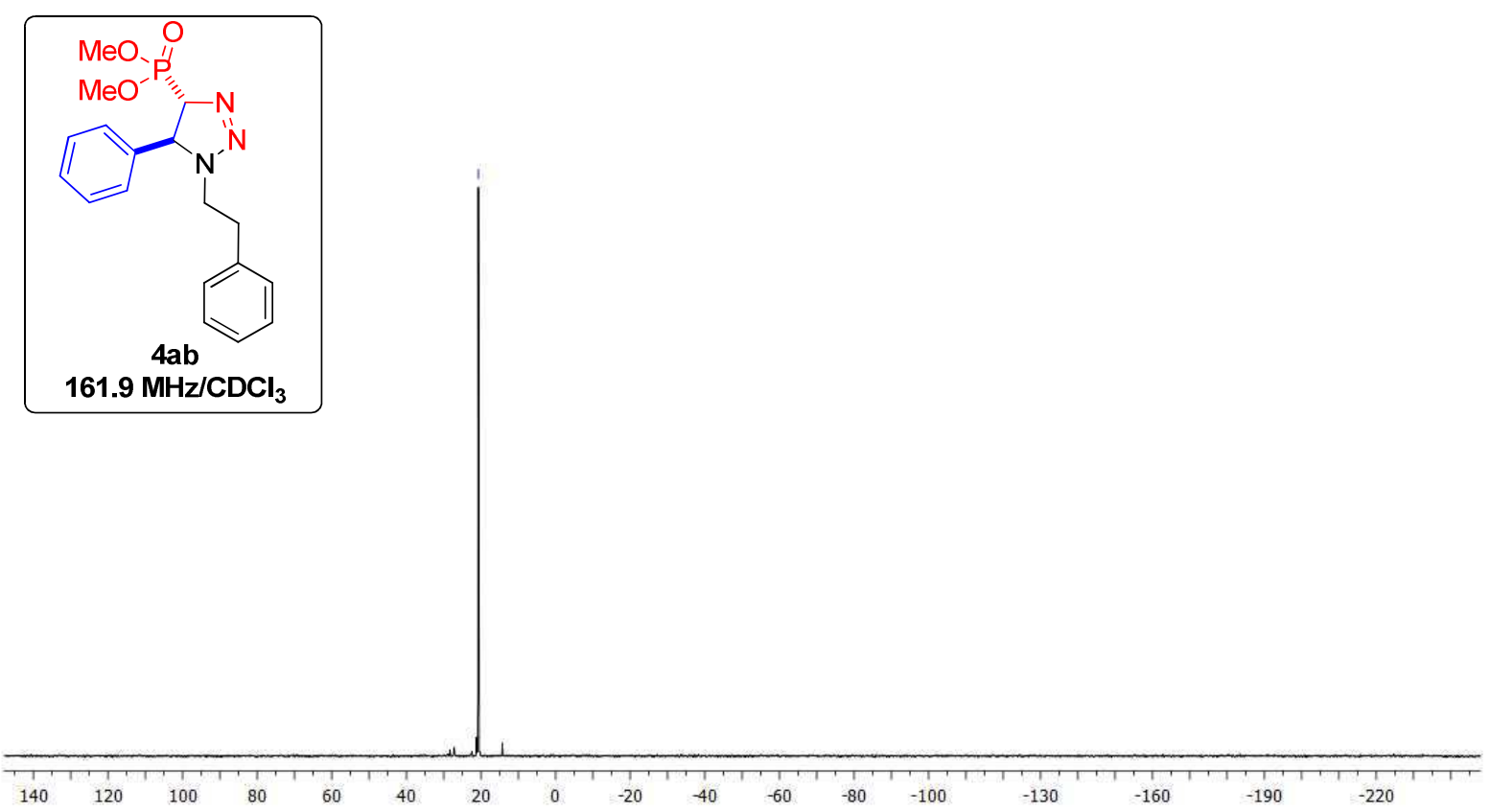

67 


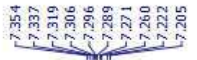

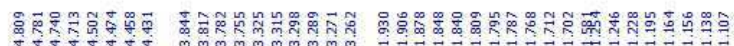<smiles>COP(OC)C1N=NN(C2CCCCC2)C1c1ccccc1</smiles>
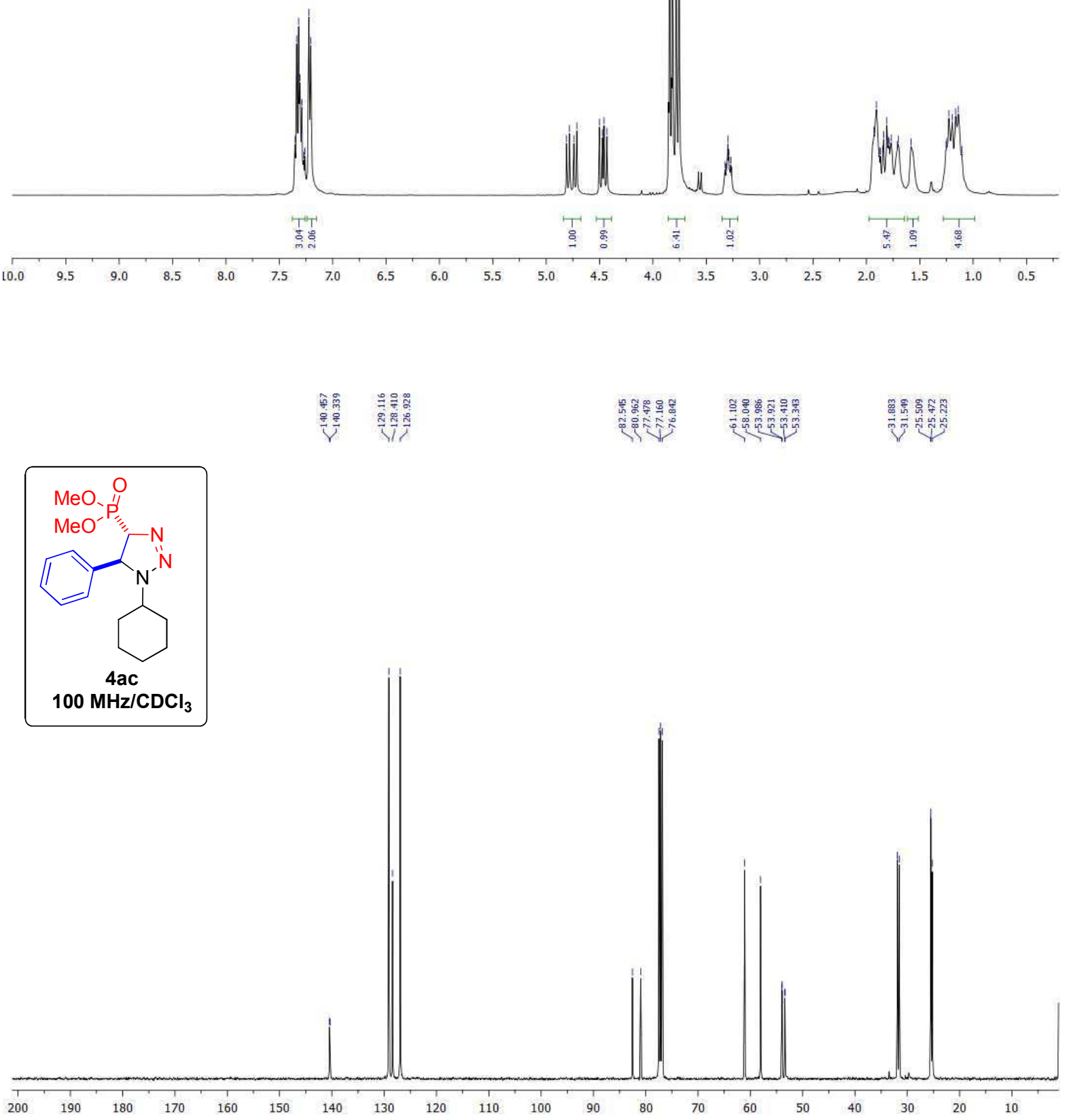


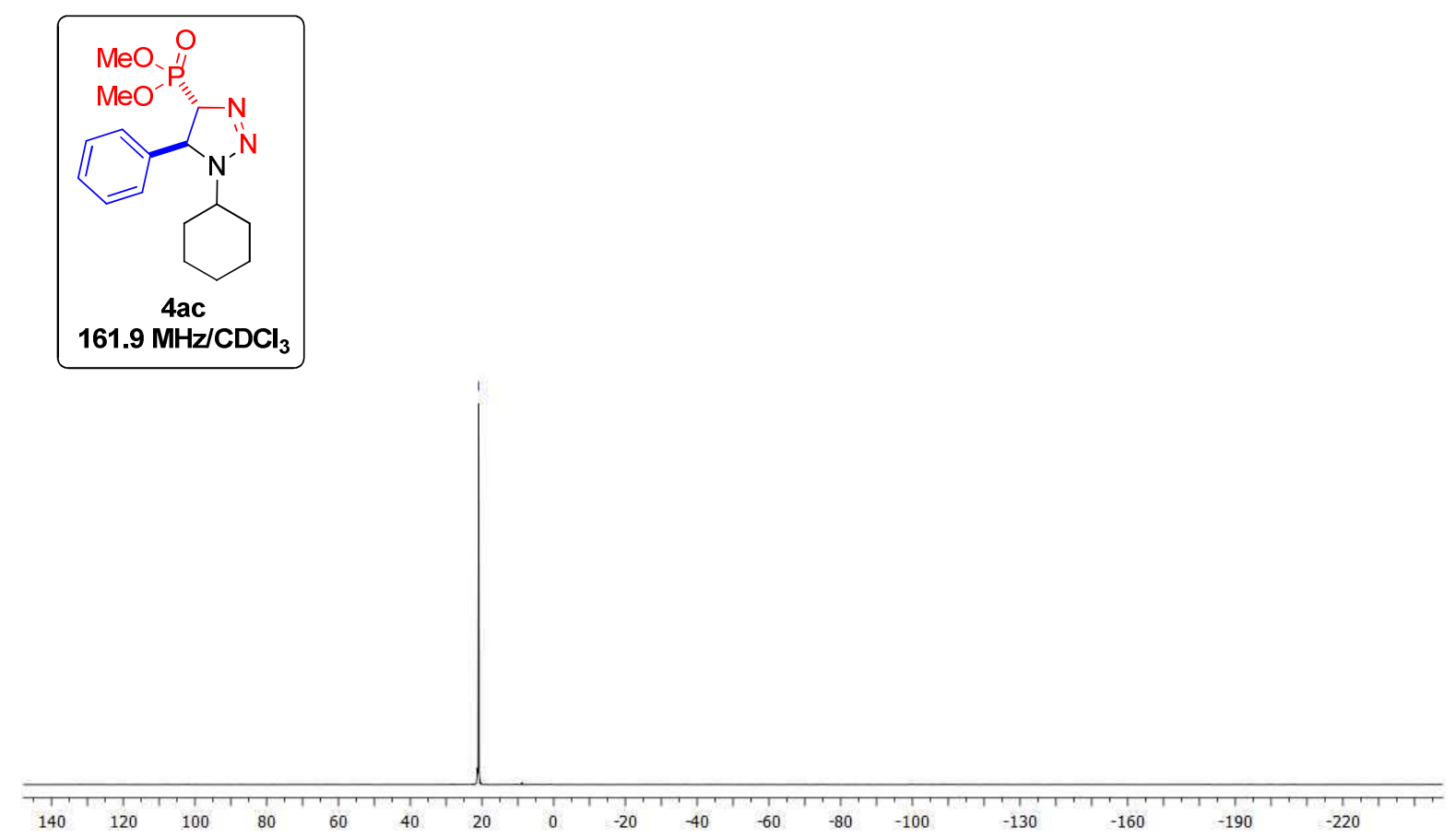

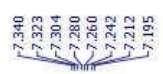

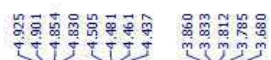

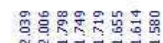

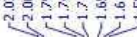

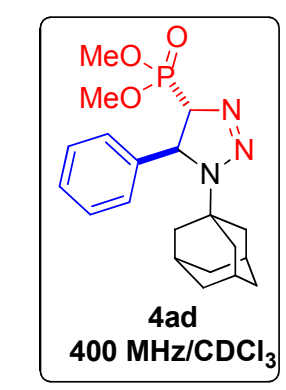

.

यู่


等等筑
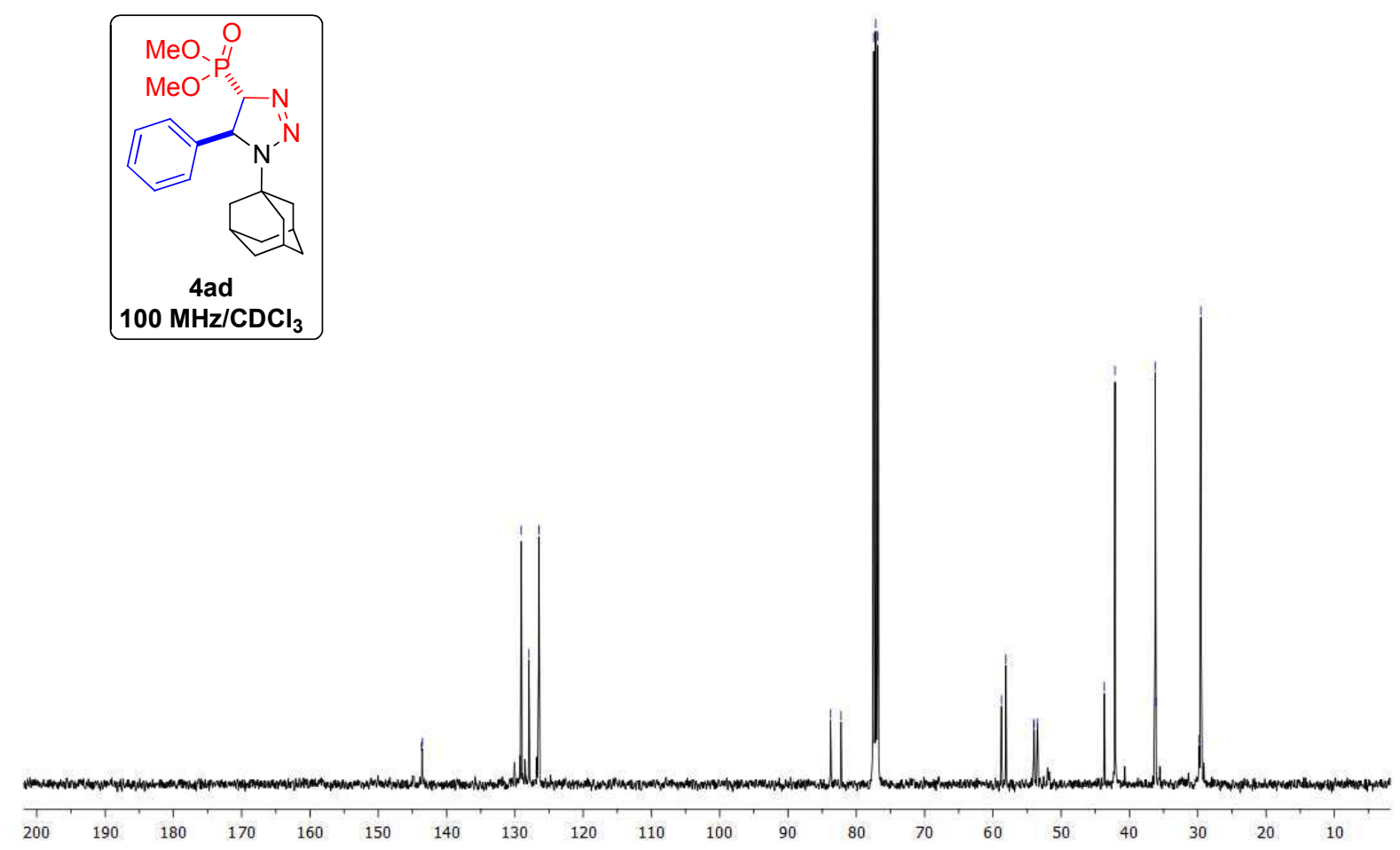

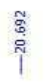

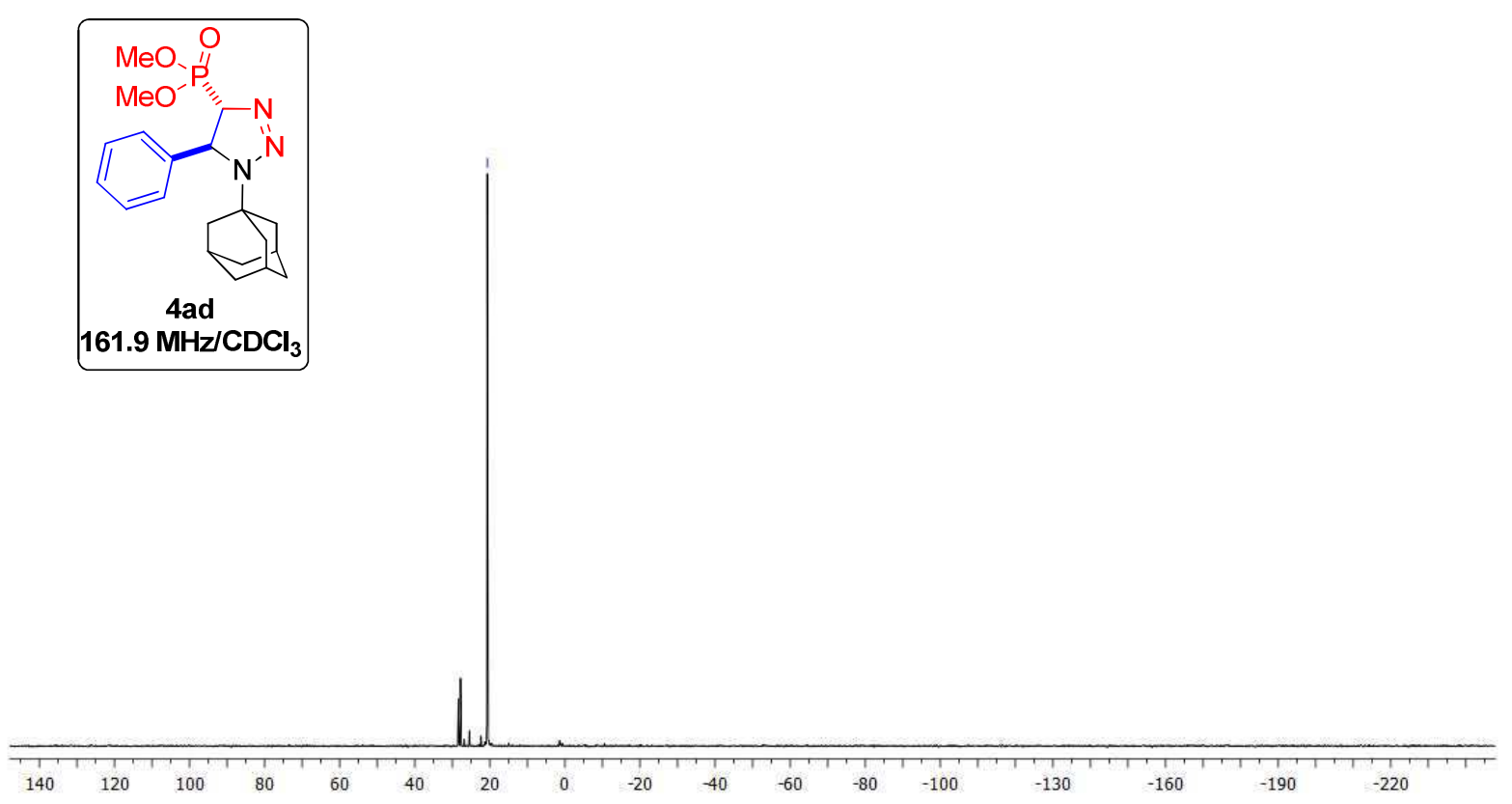

70 

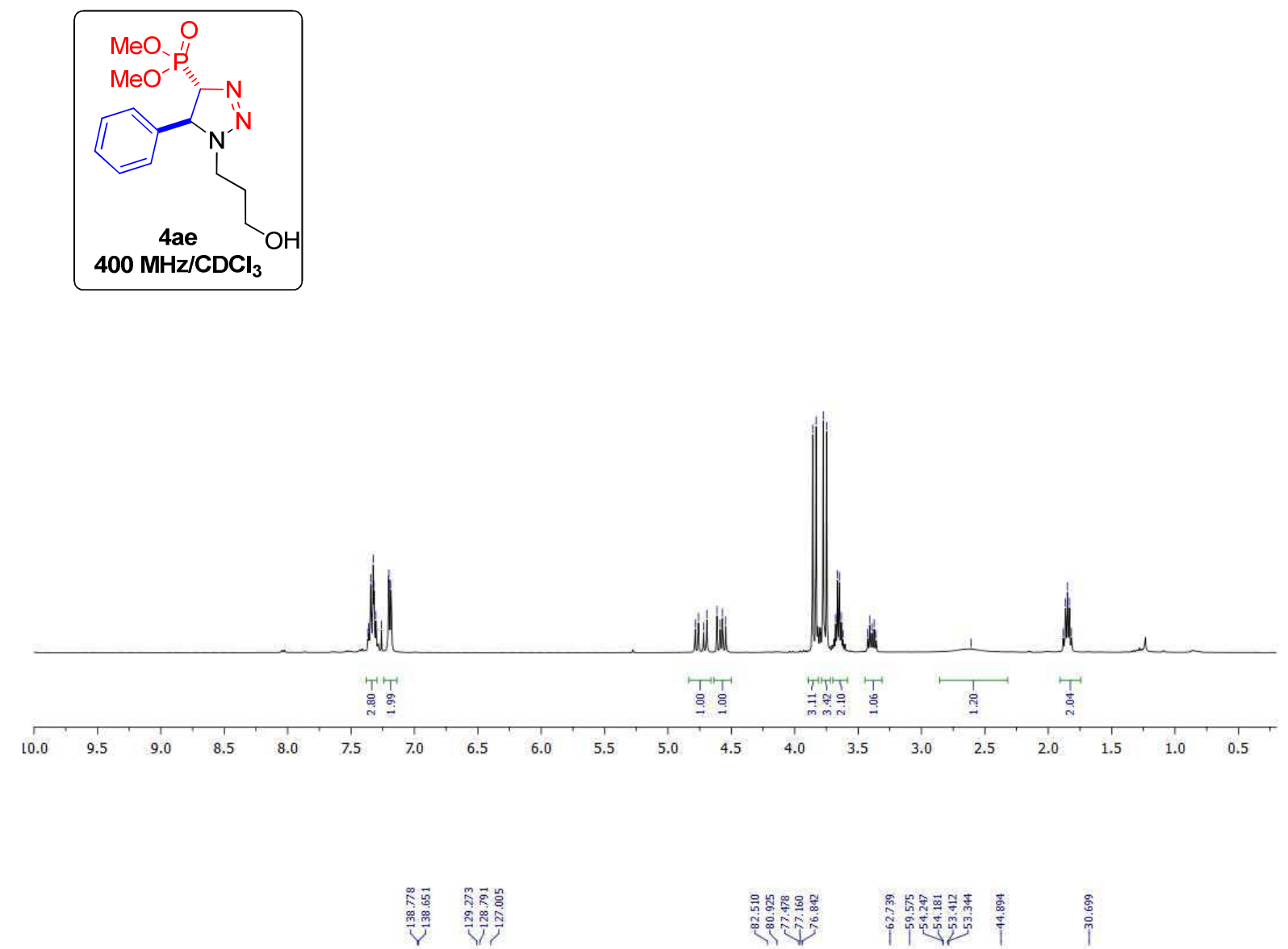

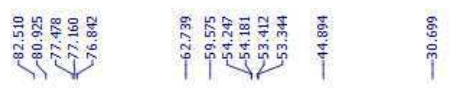
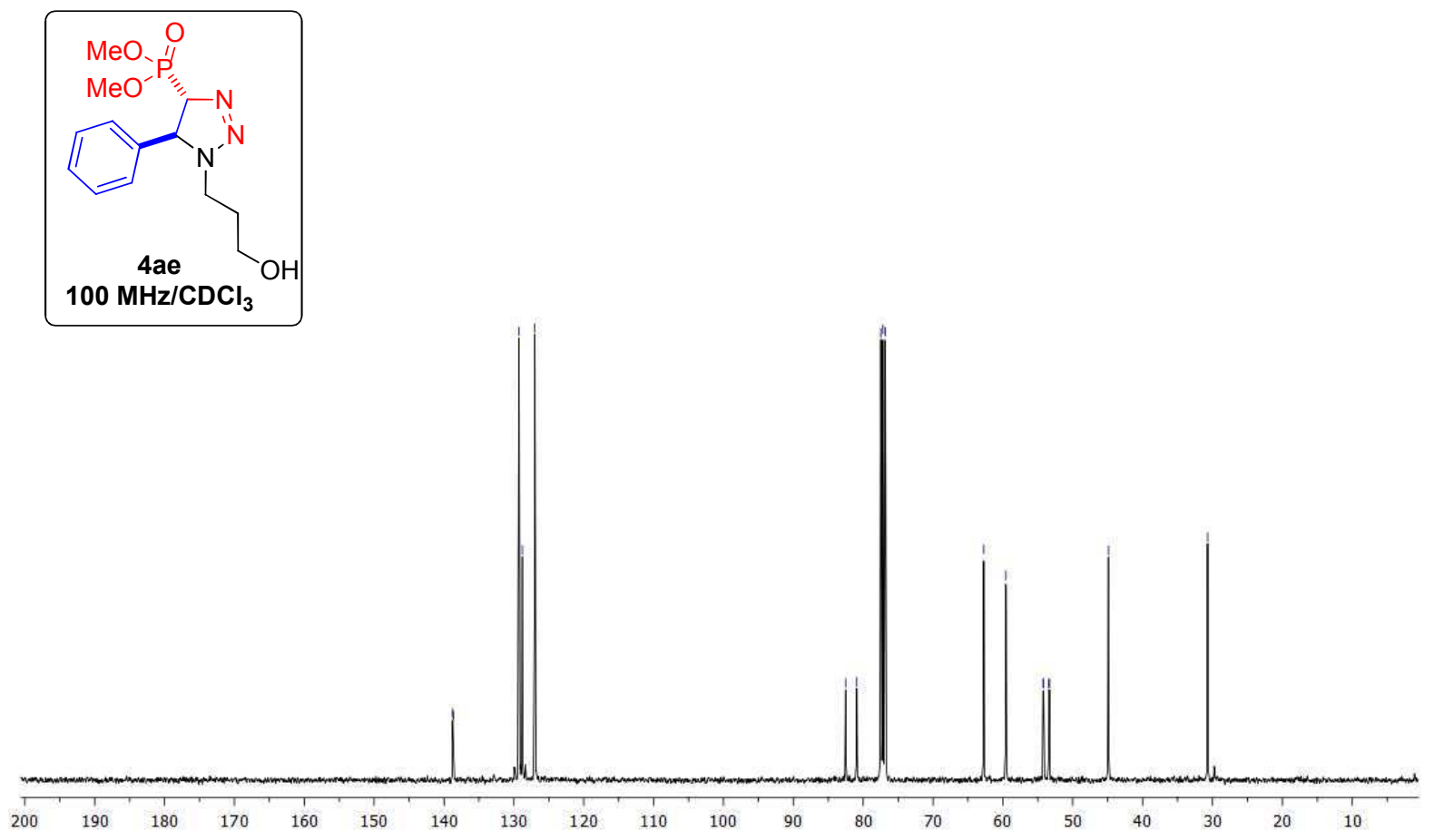


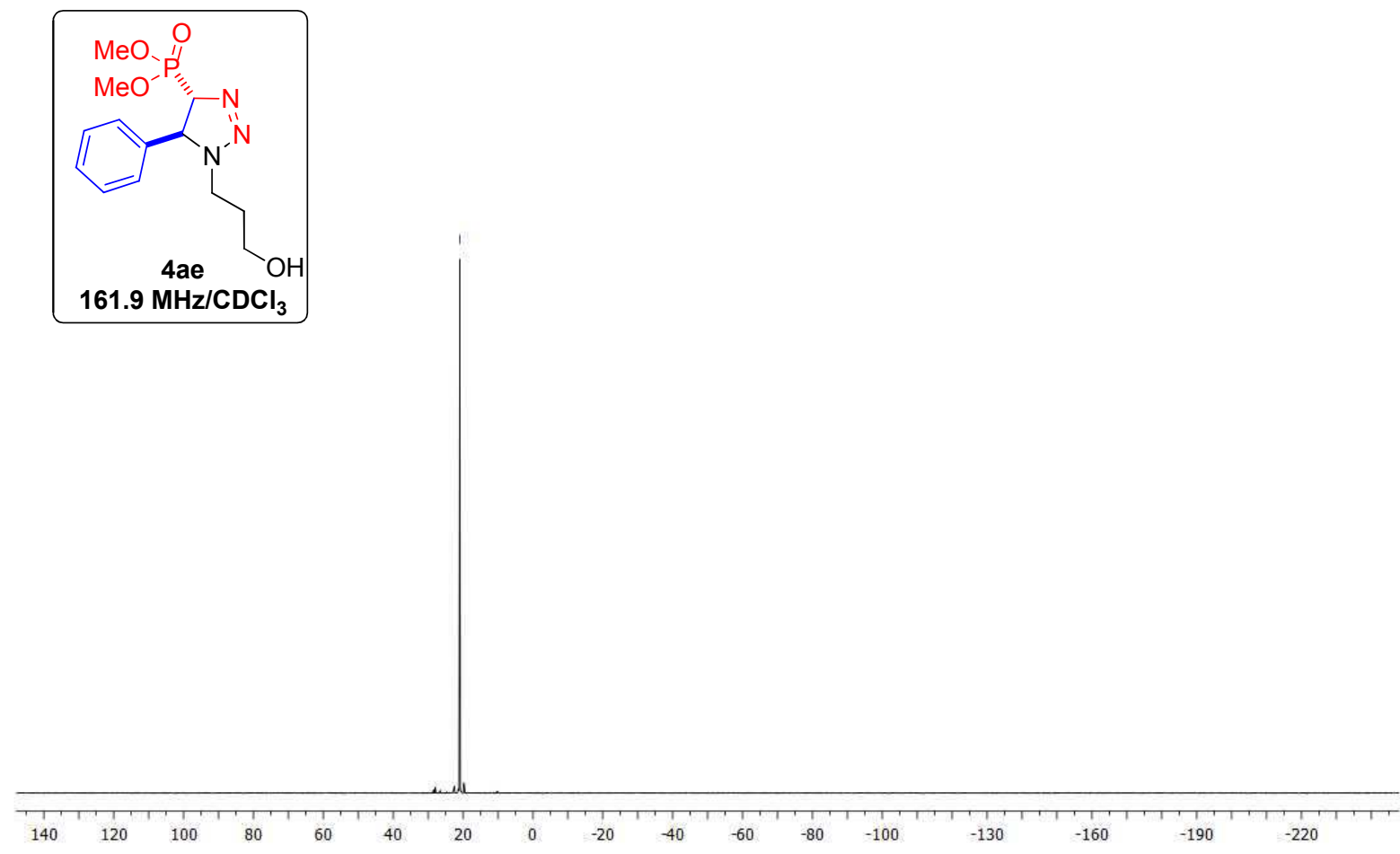

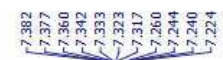

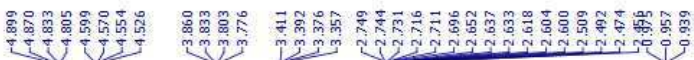

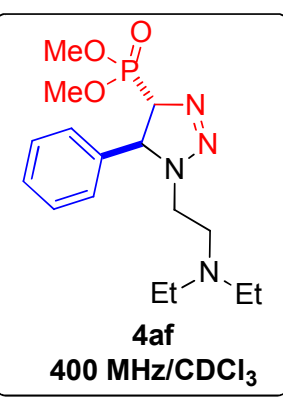

$400 \mathrm{MHz} / \mathrm{CDCl}_{3}$

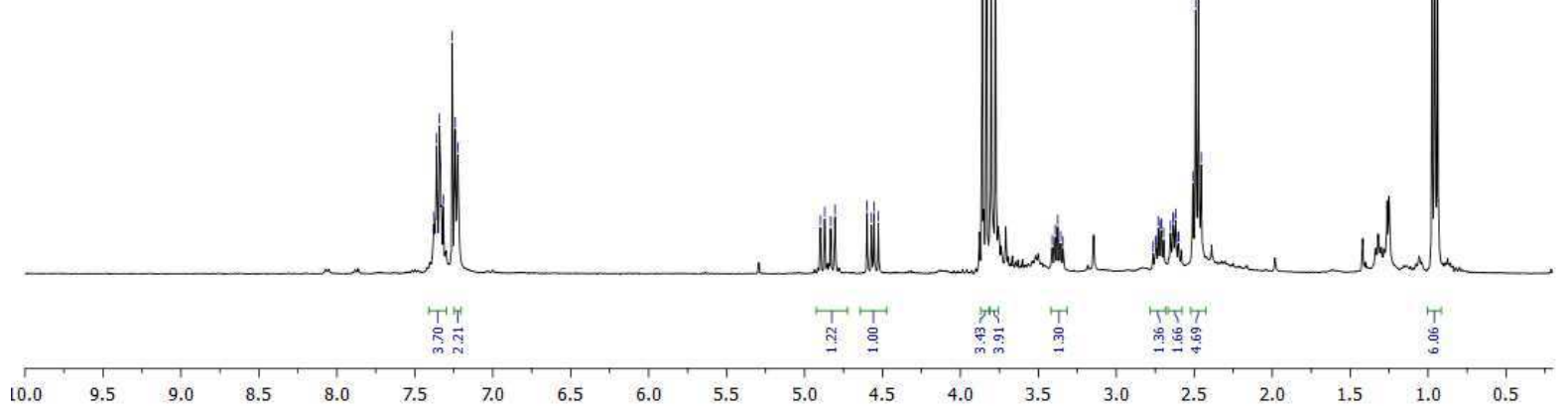



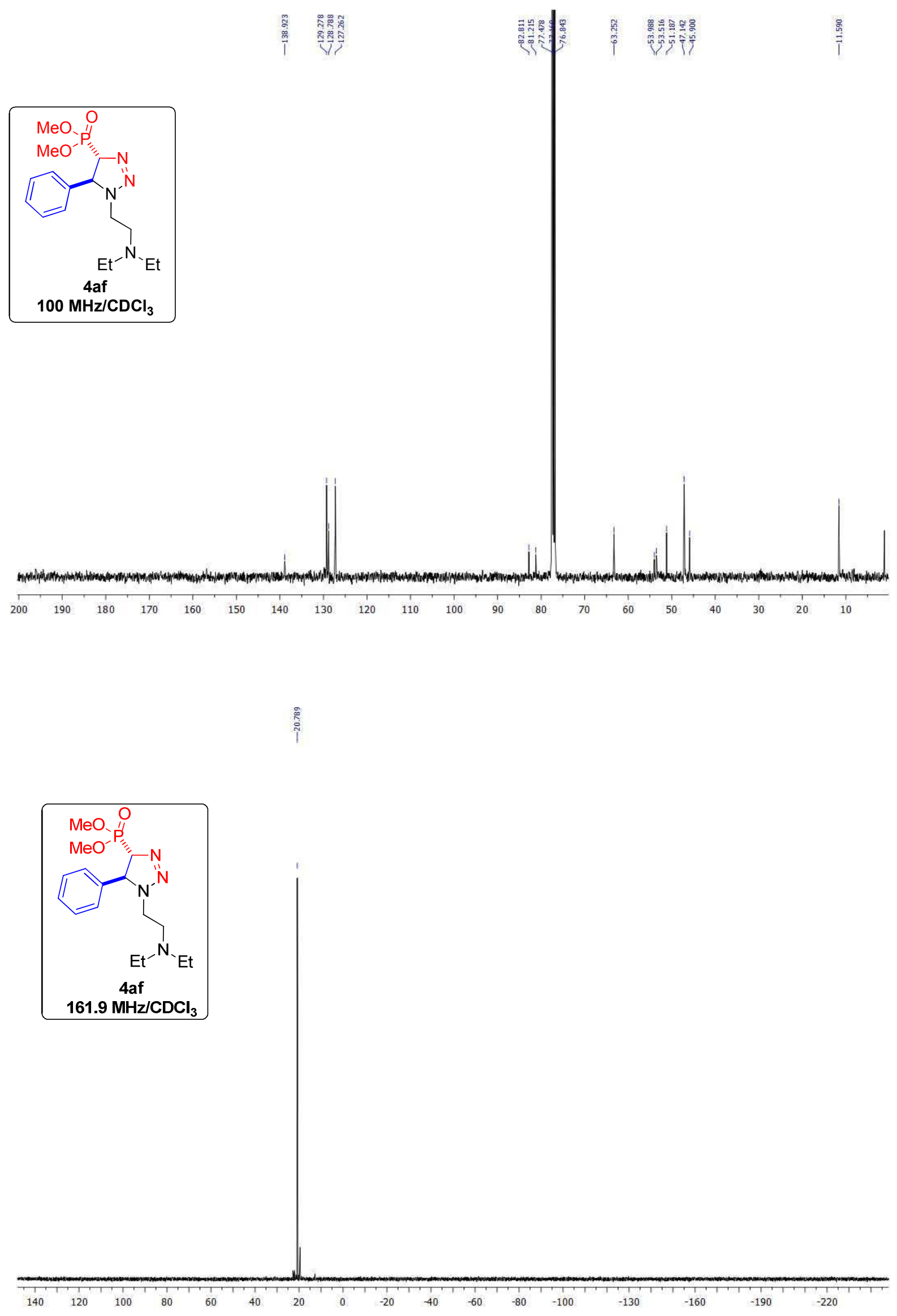

73 

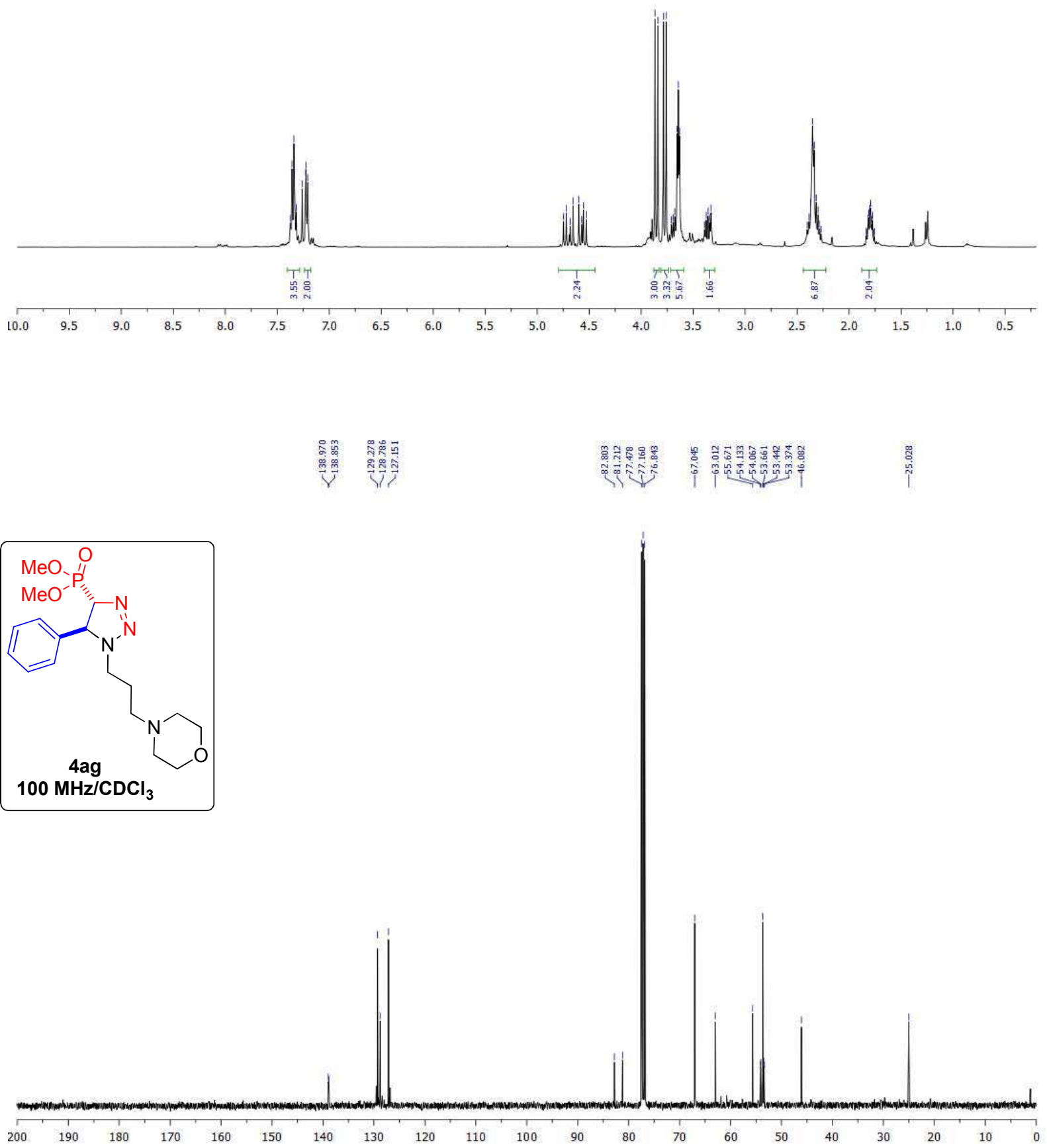

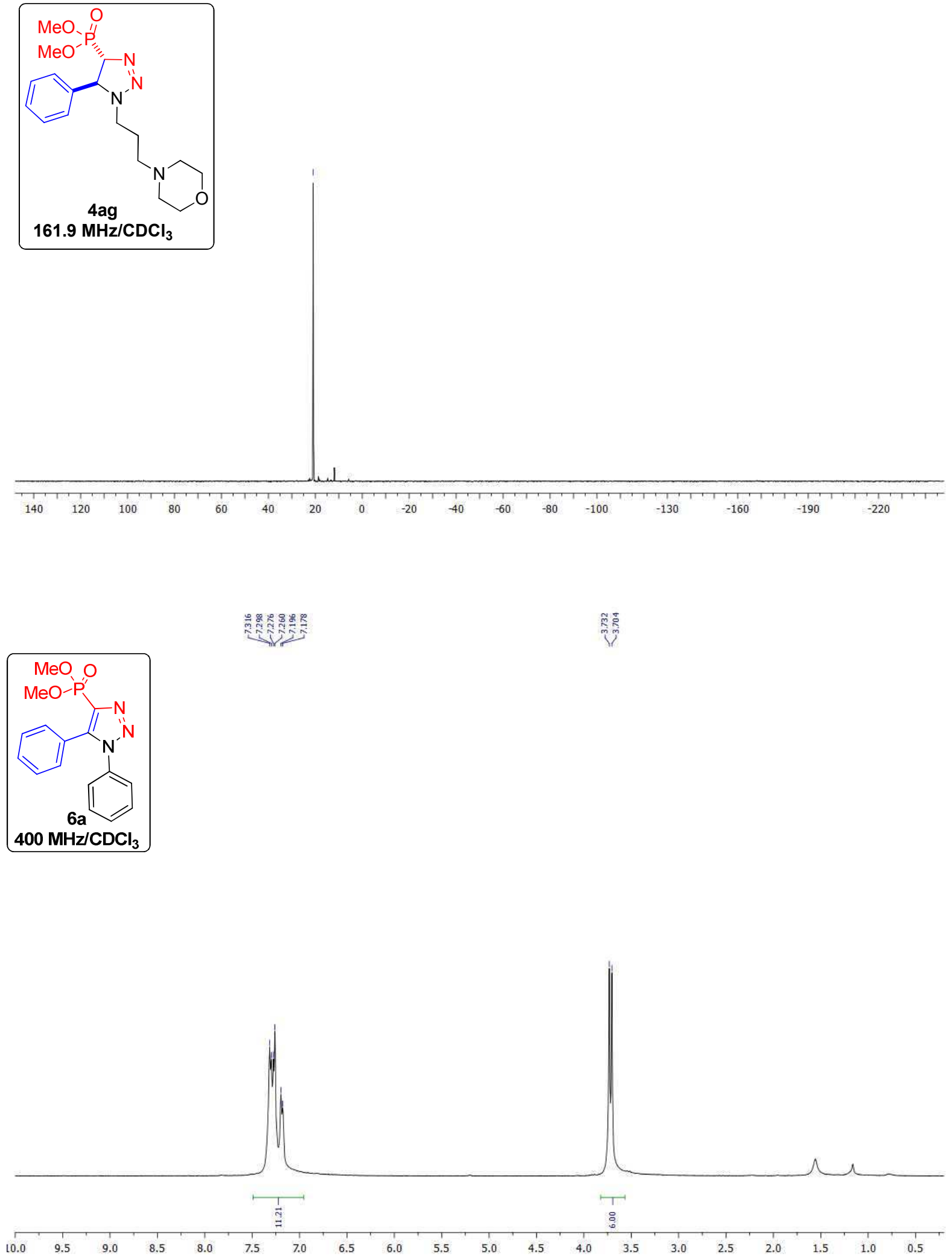

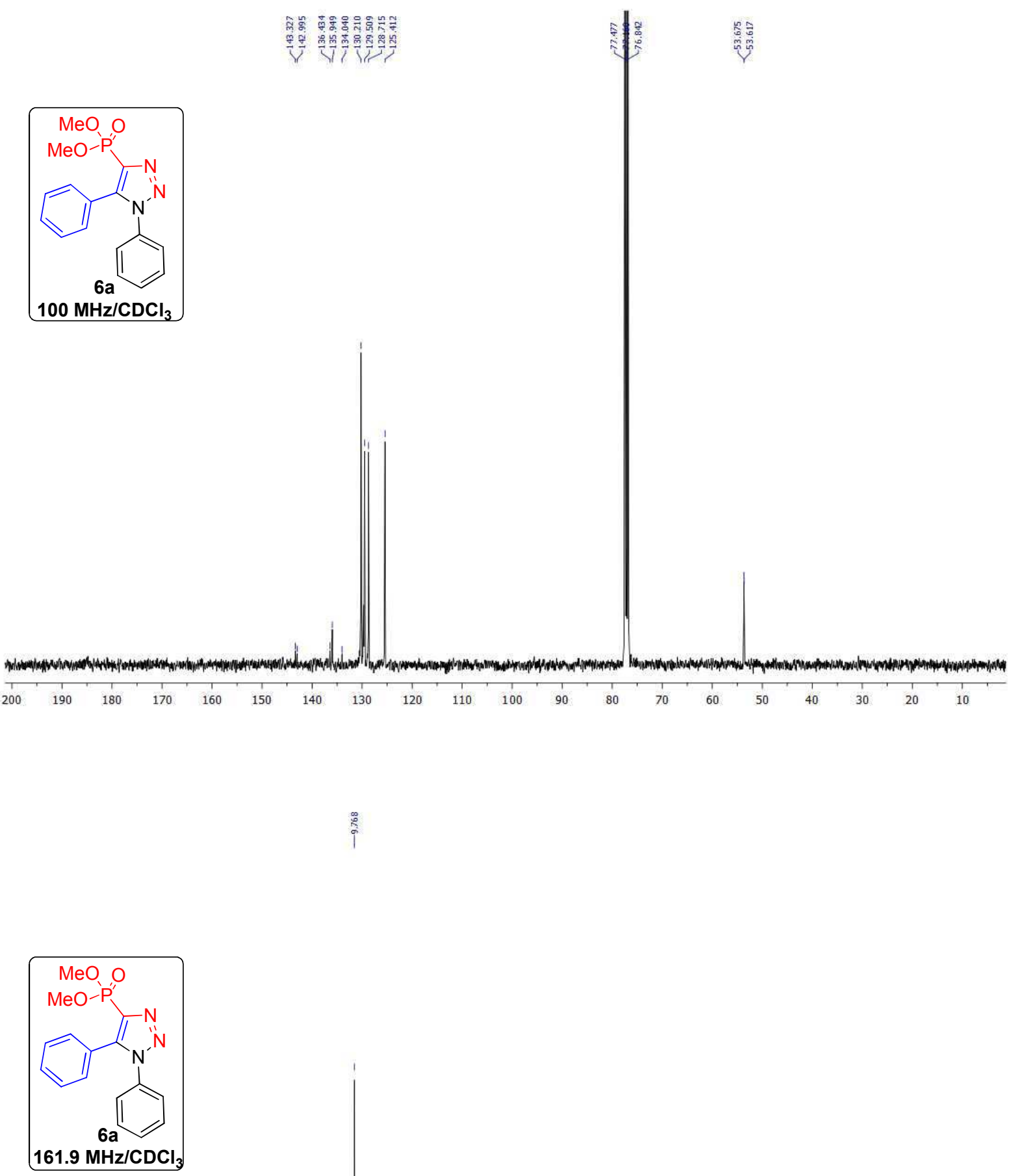


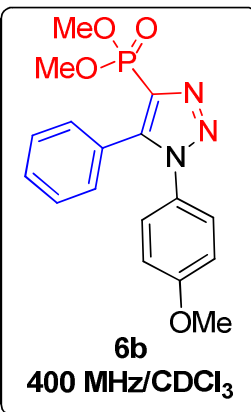

$400 \mathrm{MHz} / \mathrm{CDCl}_{3}$

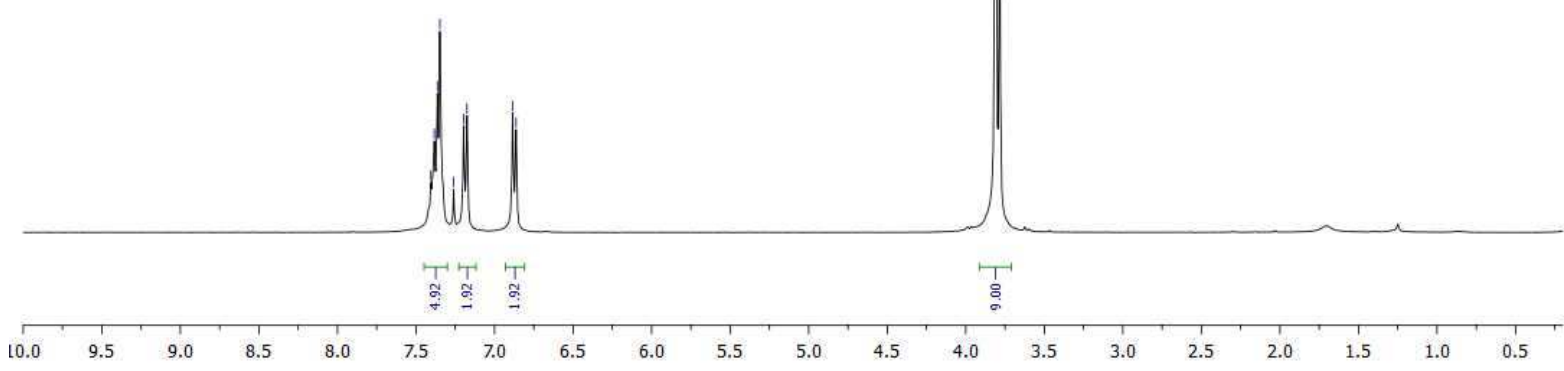

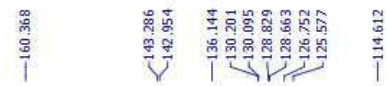
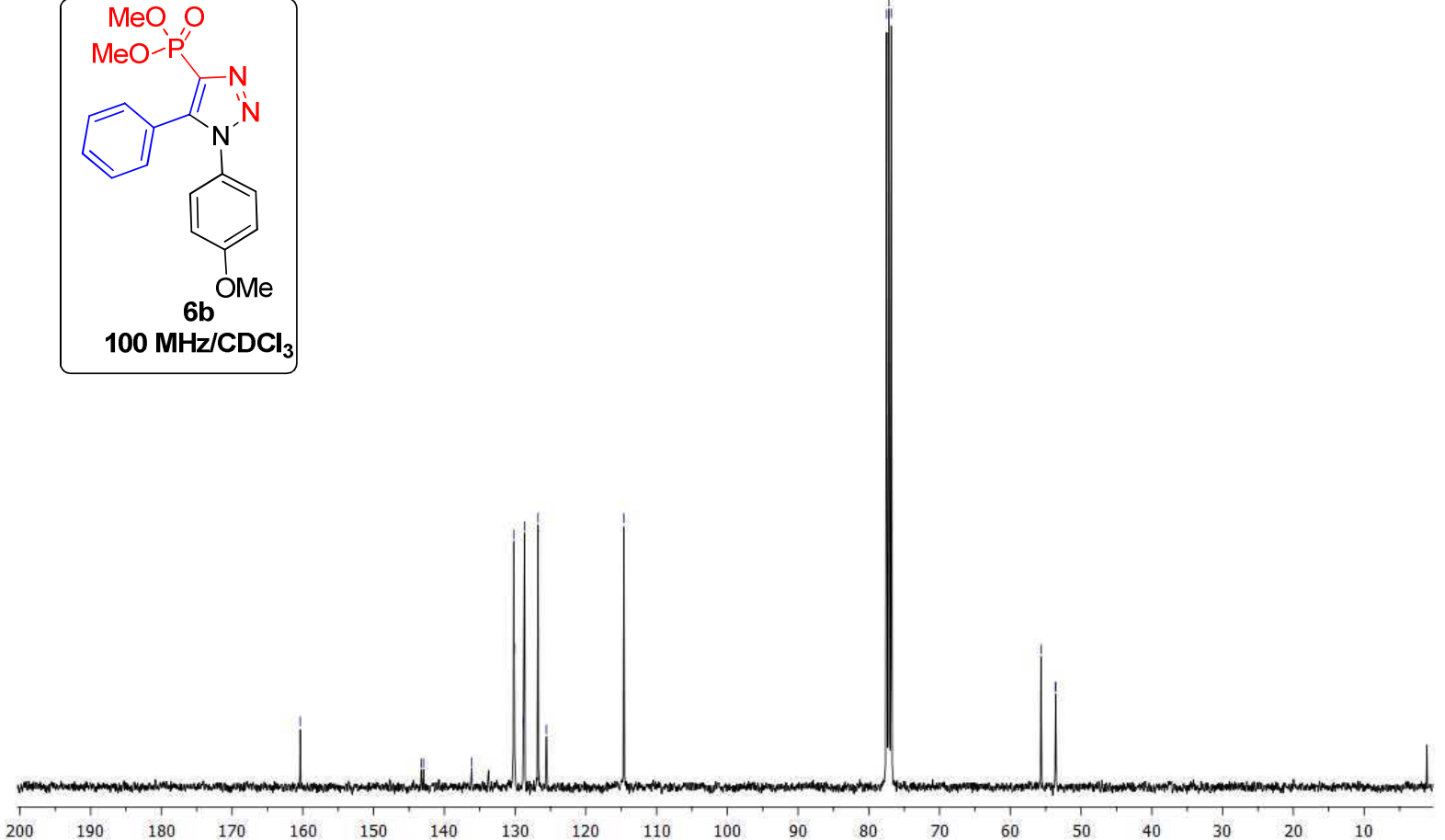

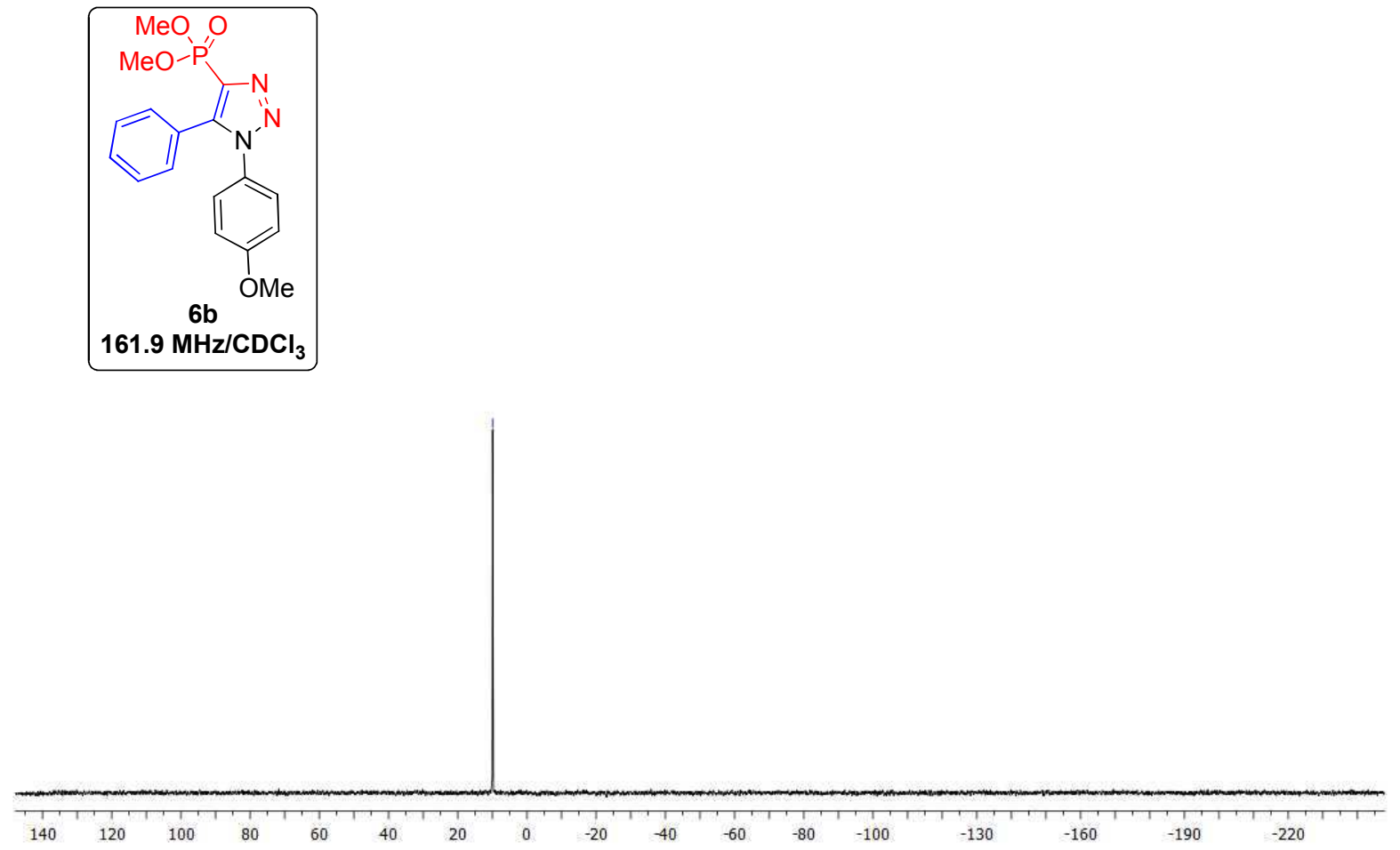

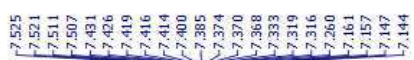
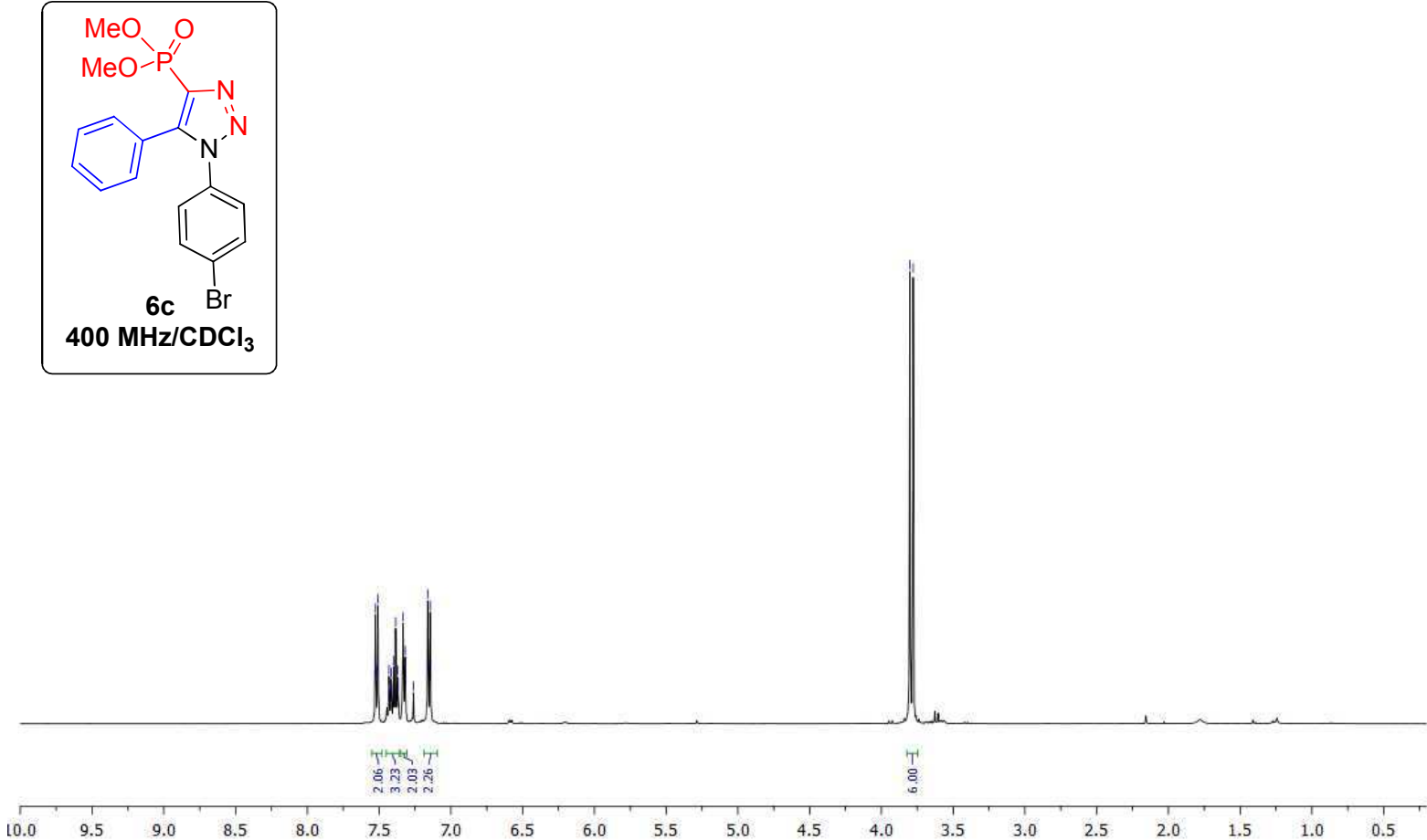

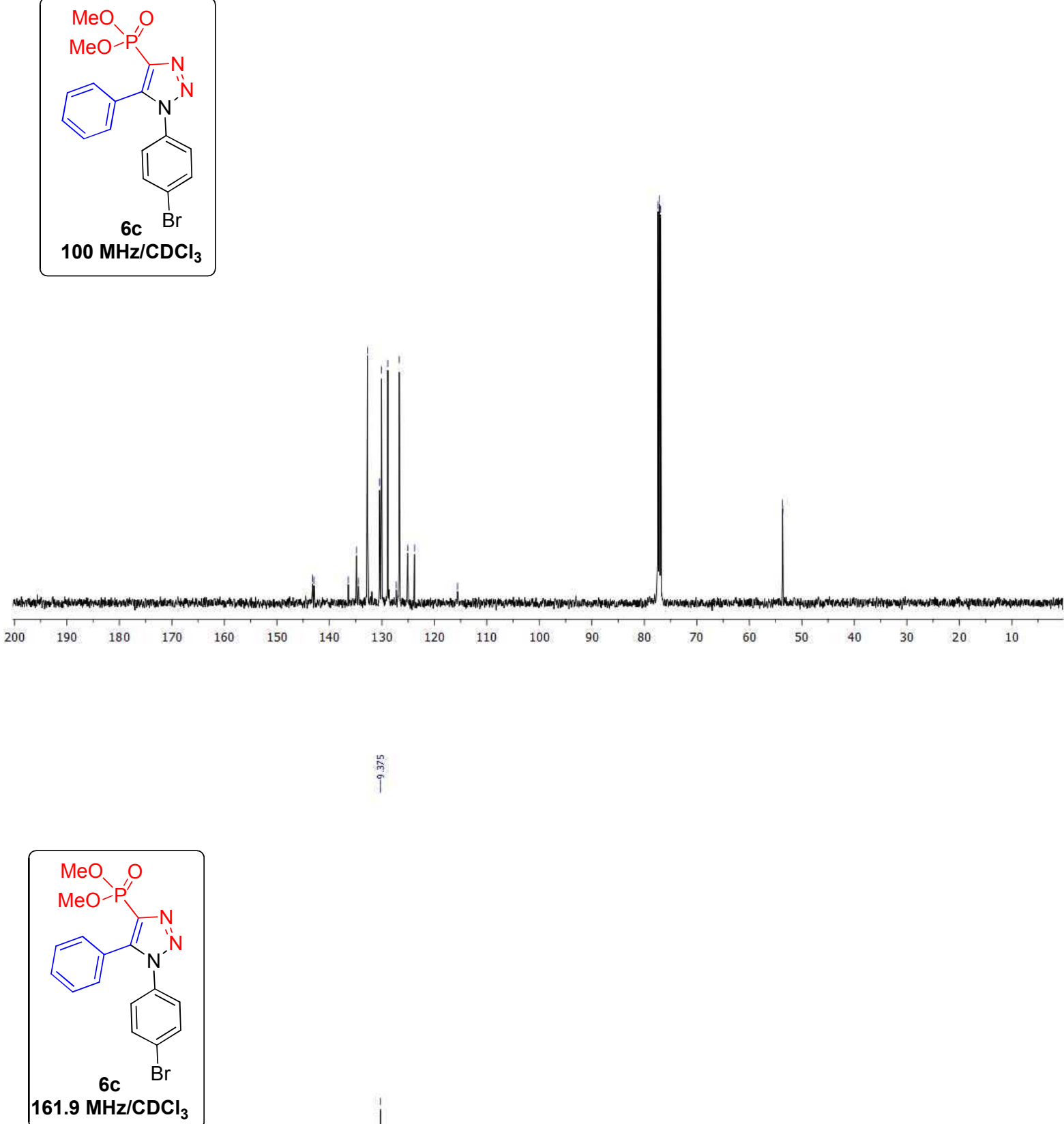

$161.9 \mathrm{MHz} / \mathrm{CDCl}_{3}$

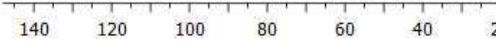

$\begin{array}{lllllllll}1 & 1 & 1 & 1 & 1 & 1 & 1 & 1 & 1 \\ 0 & -20 & -40 & -60 & -80 & -100\end{array}$ $-130$ $-190$ 

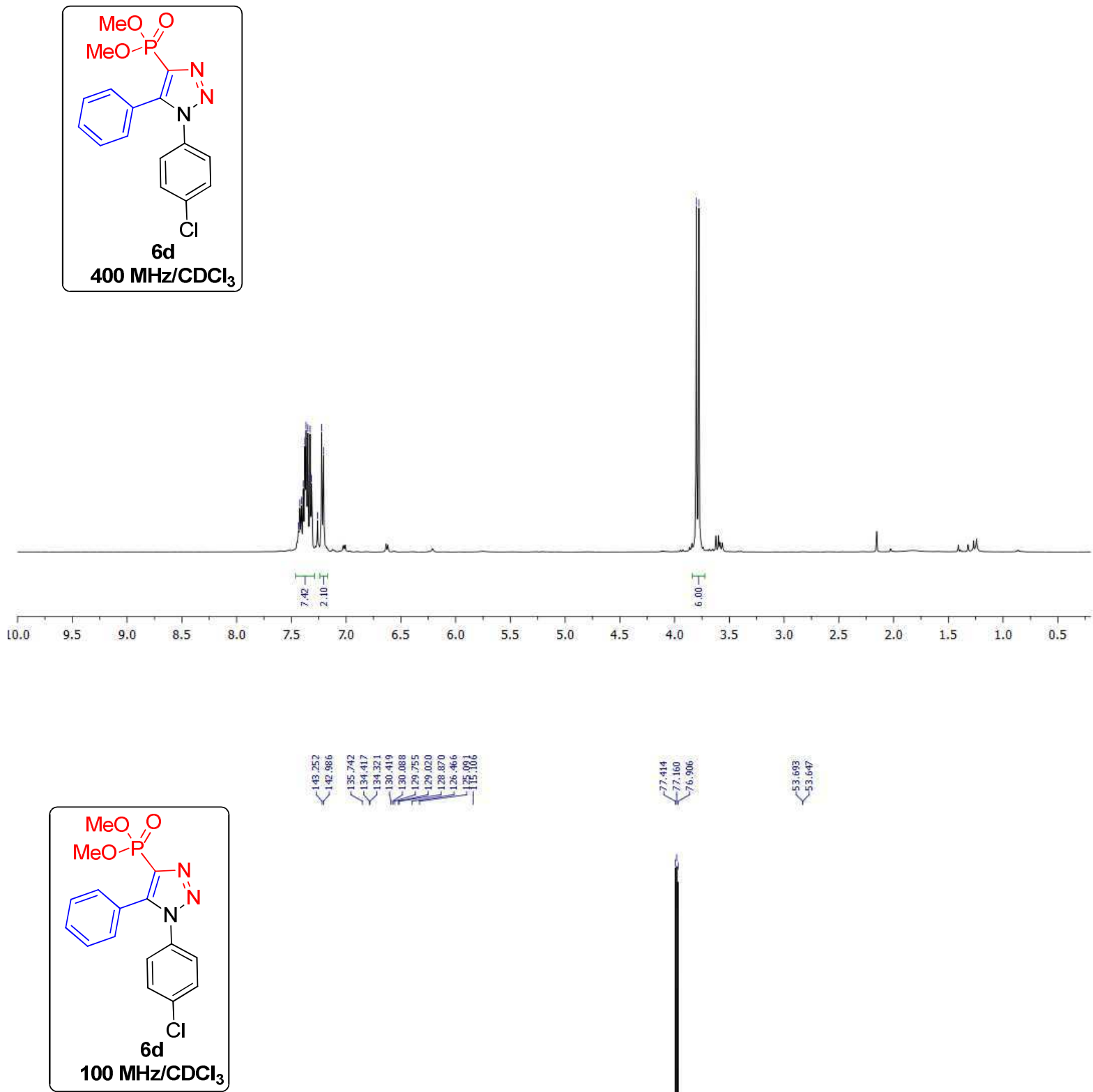

포영

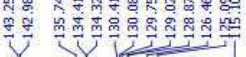

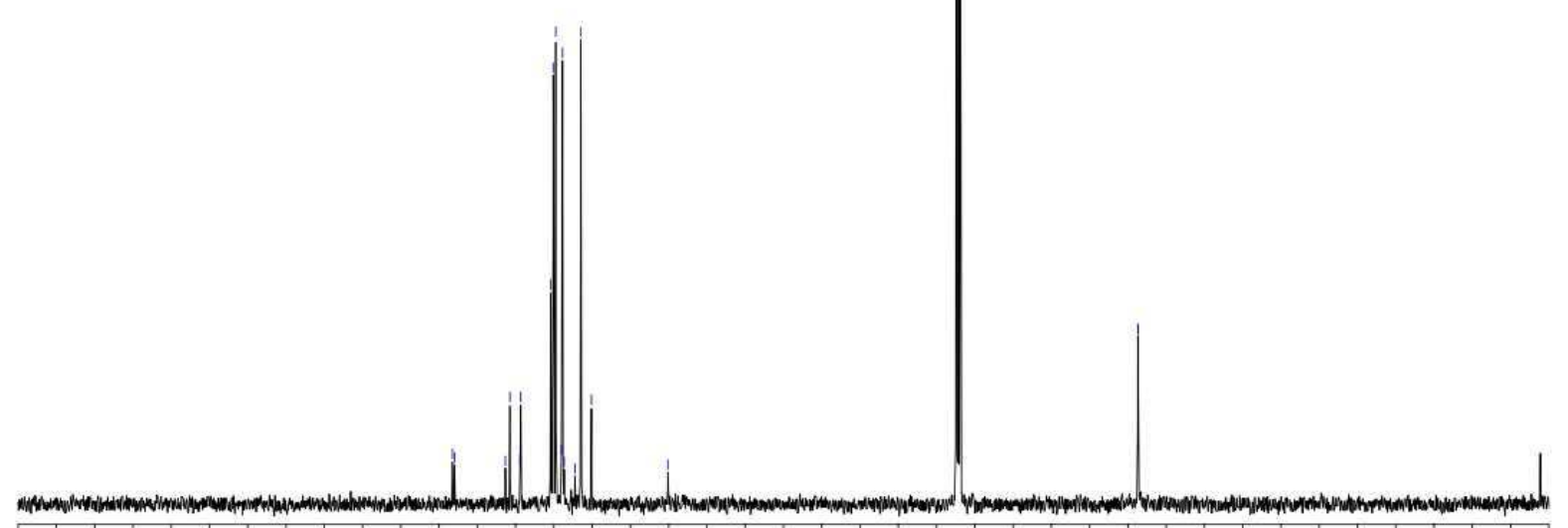

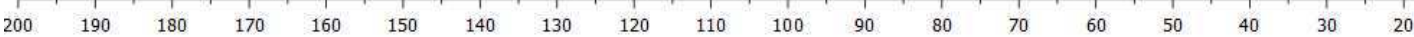




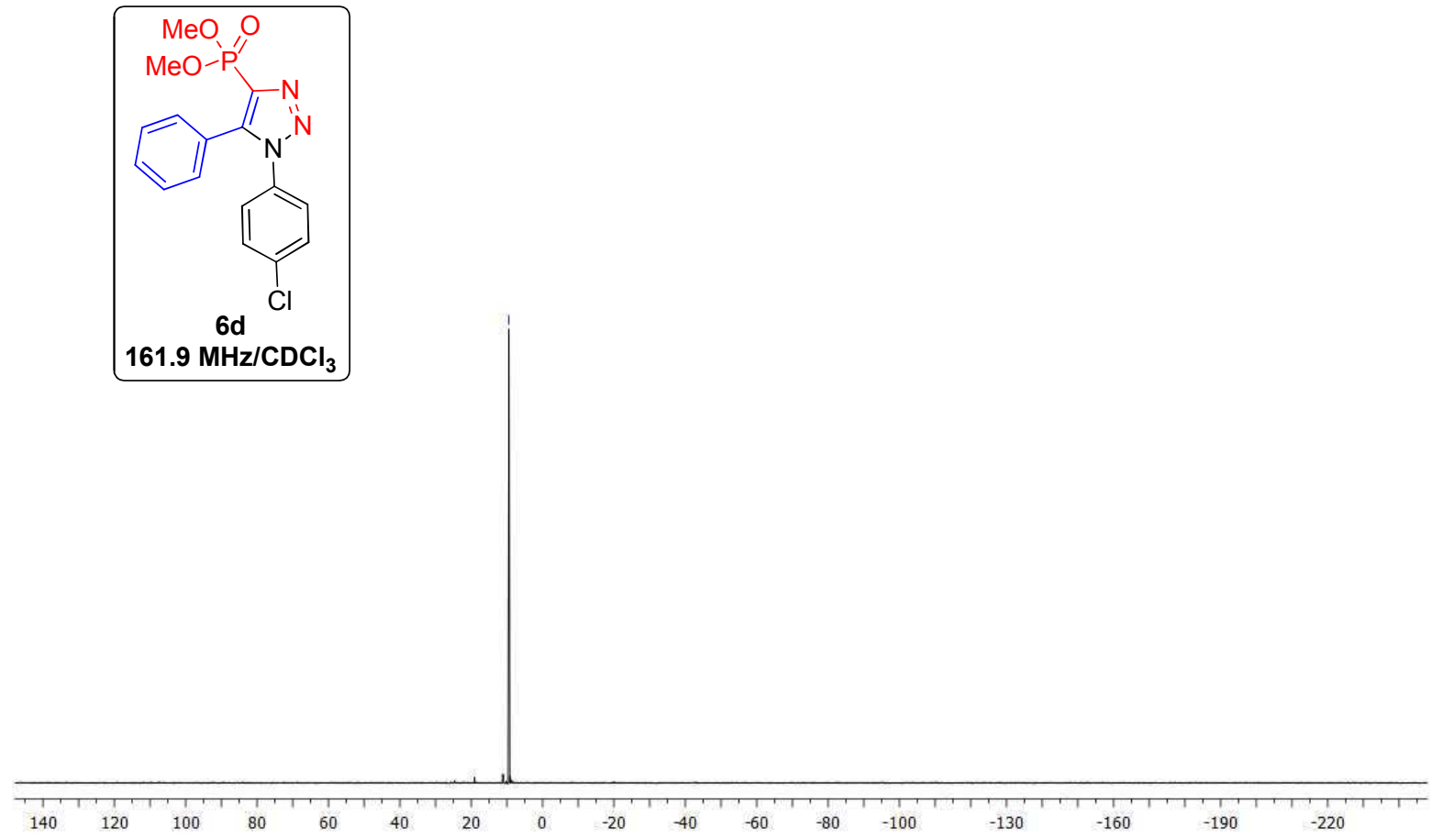

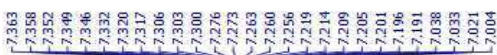
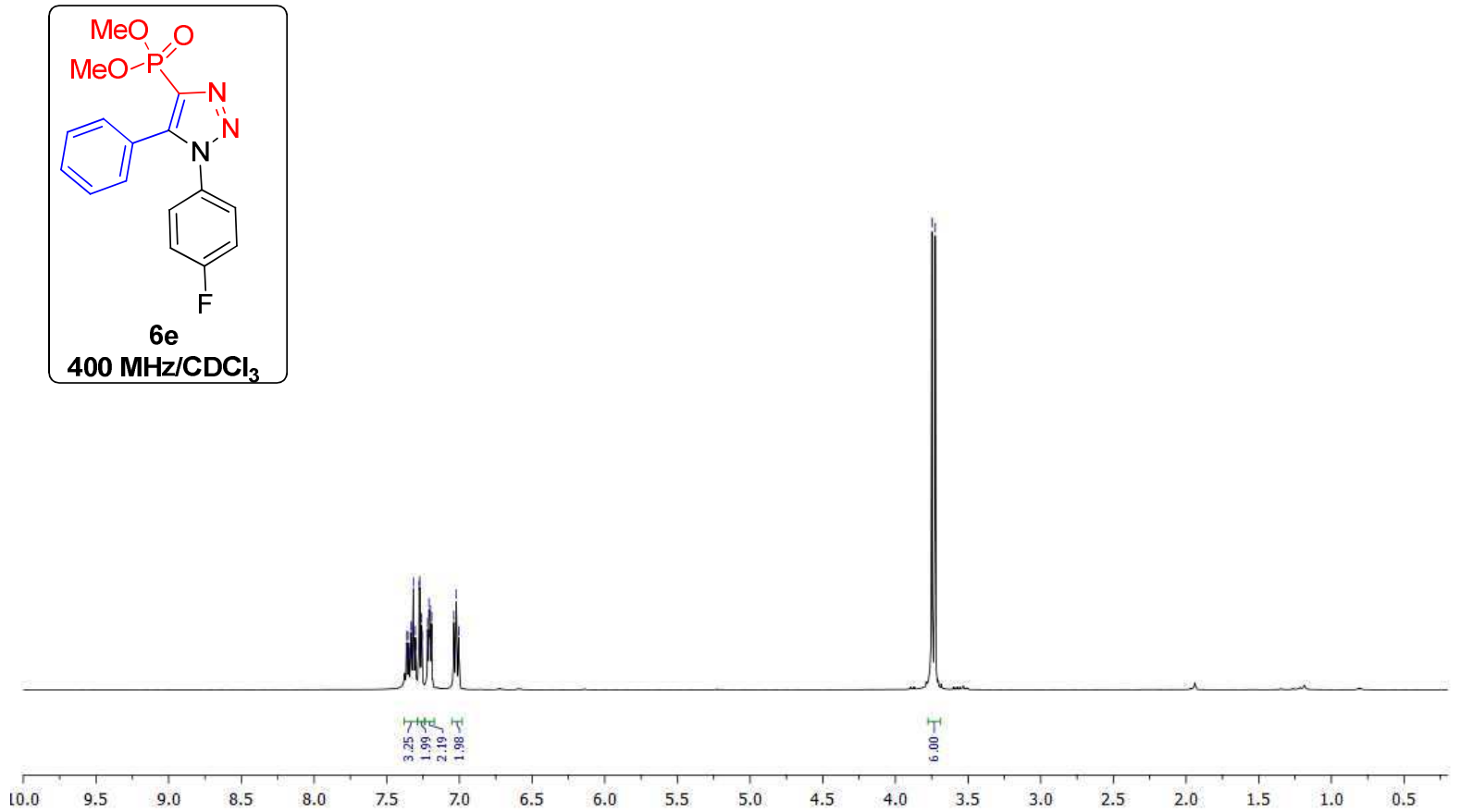

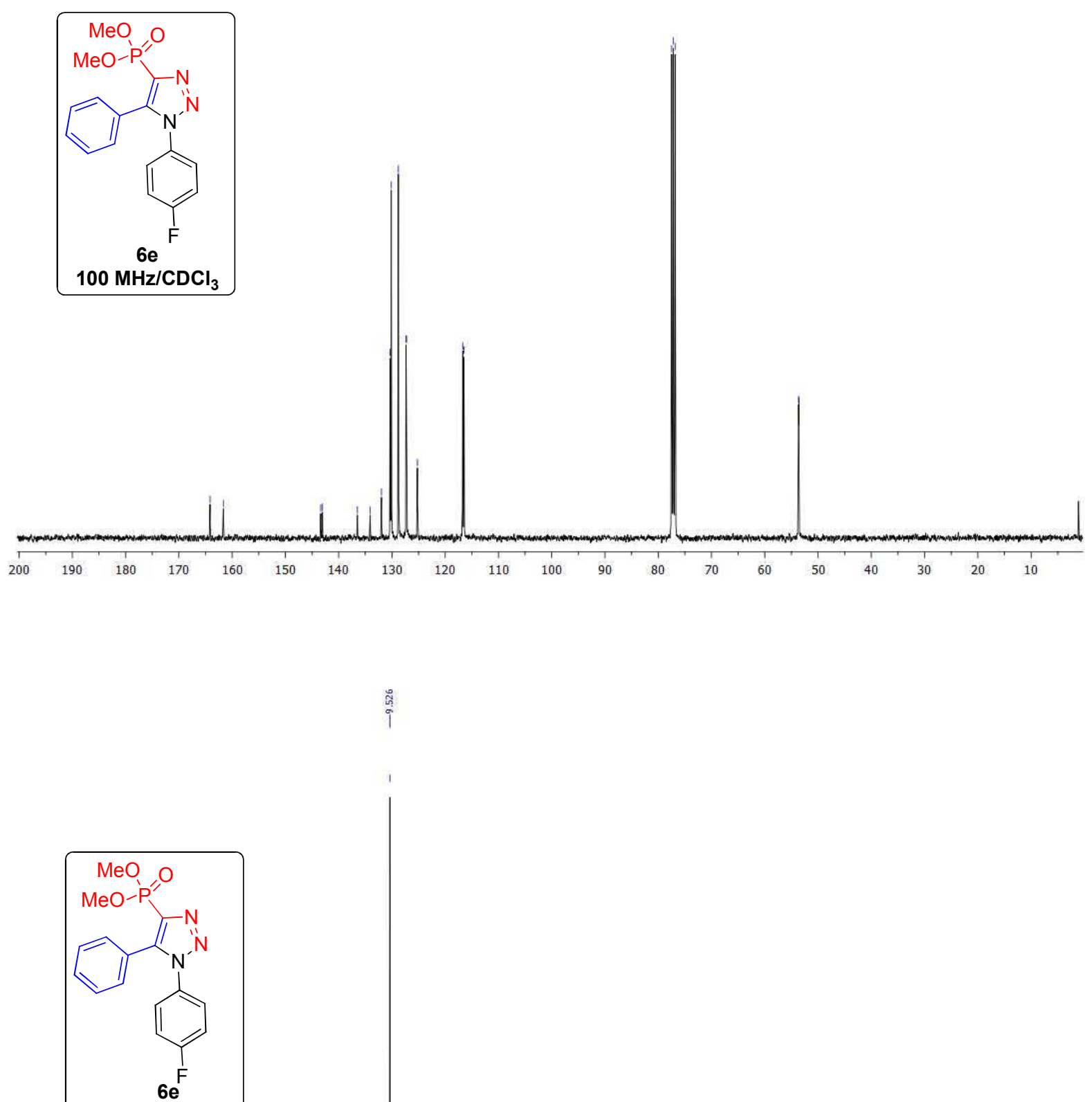

161.9 MHz/CDCl

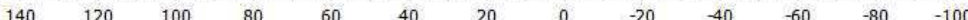



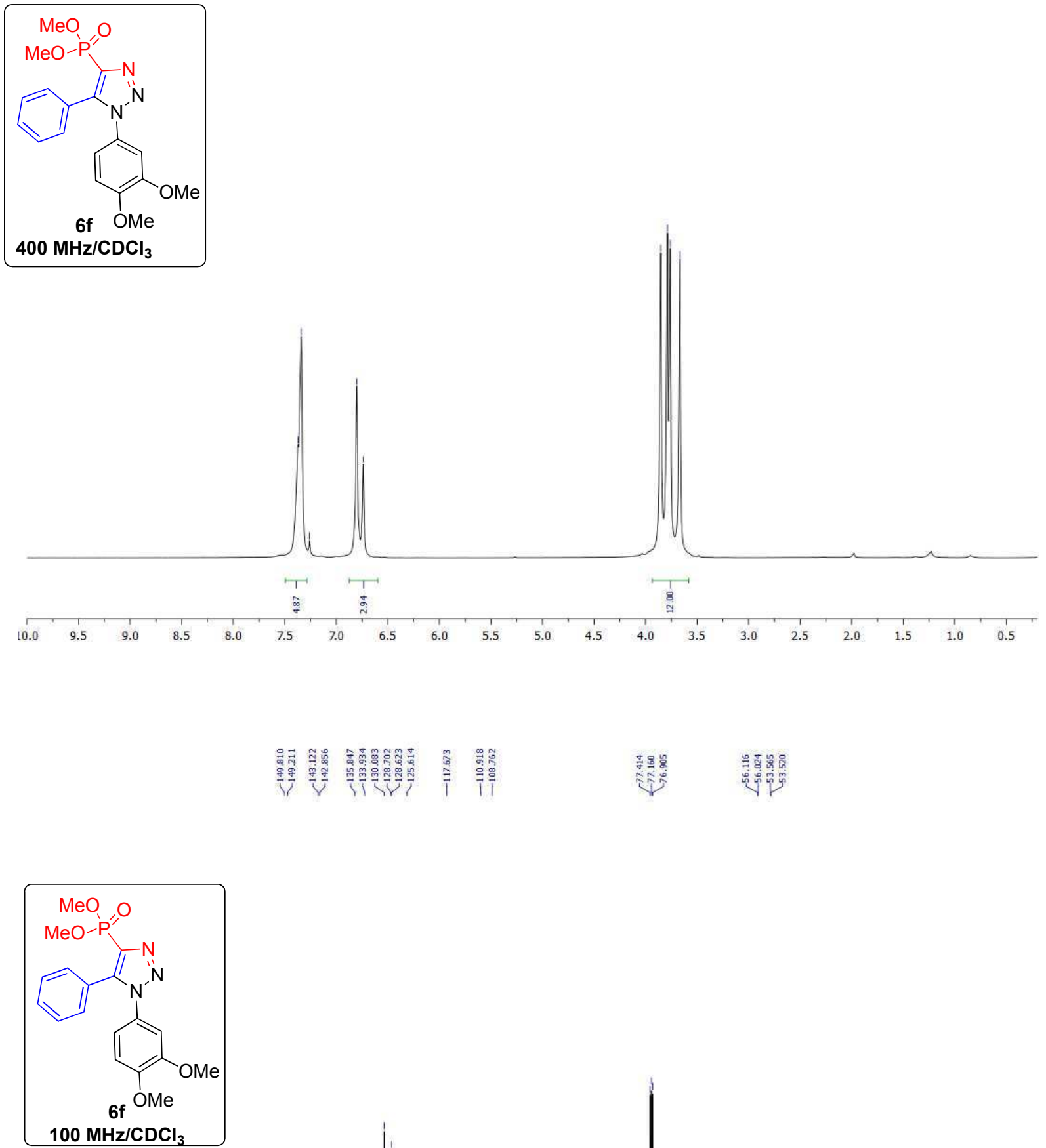

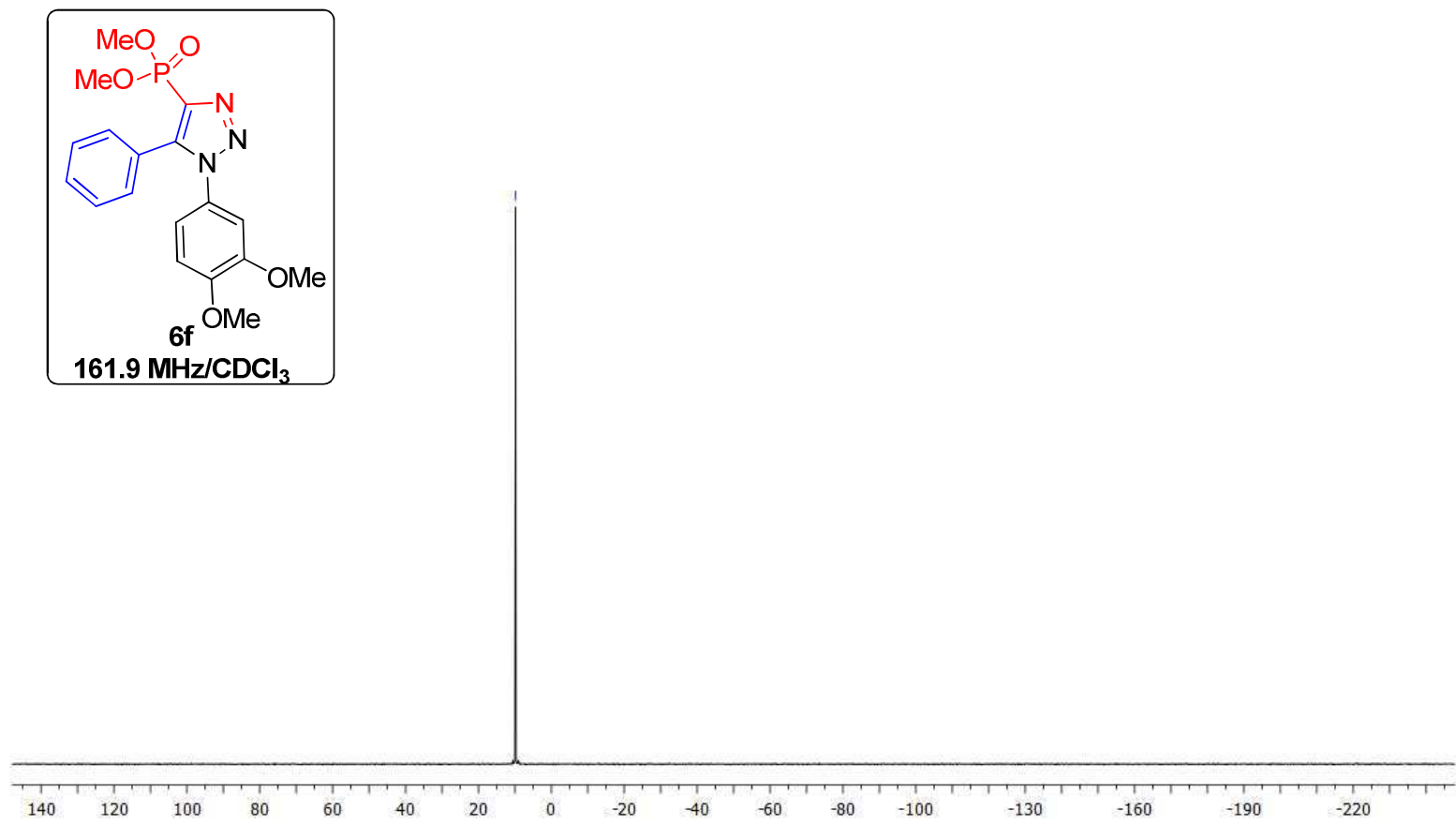

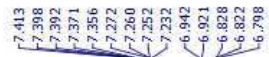
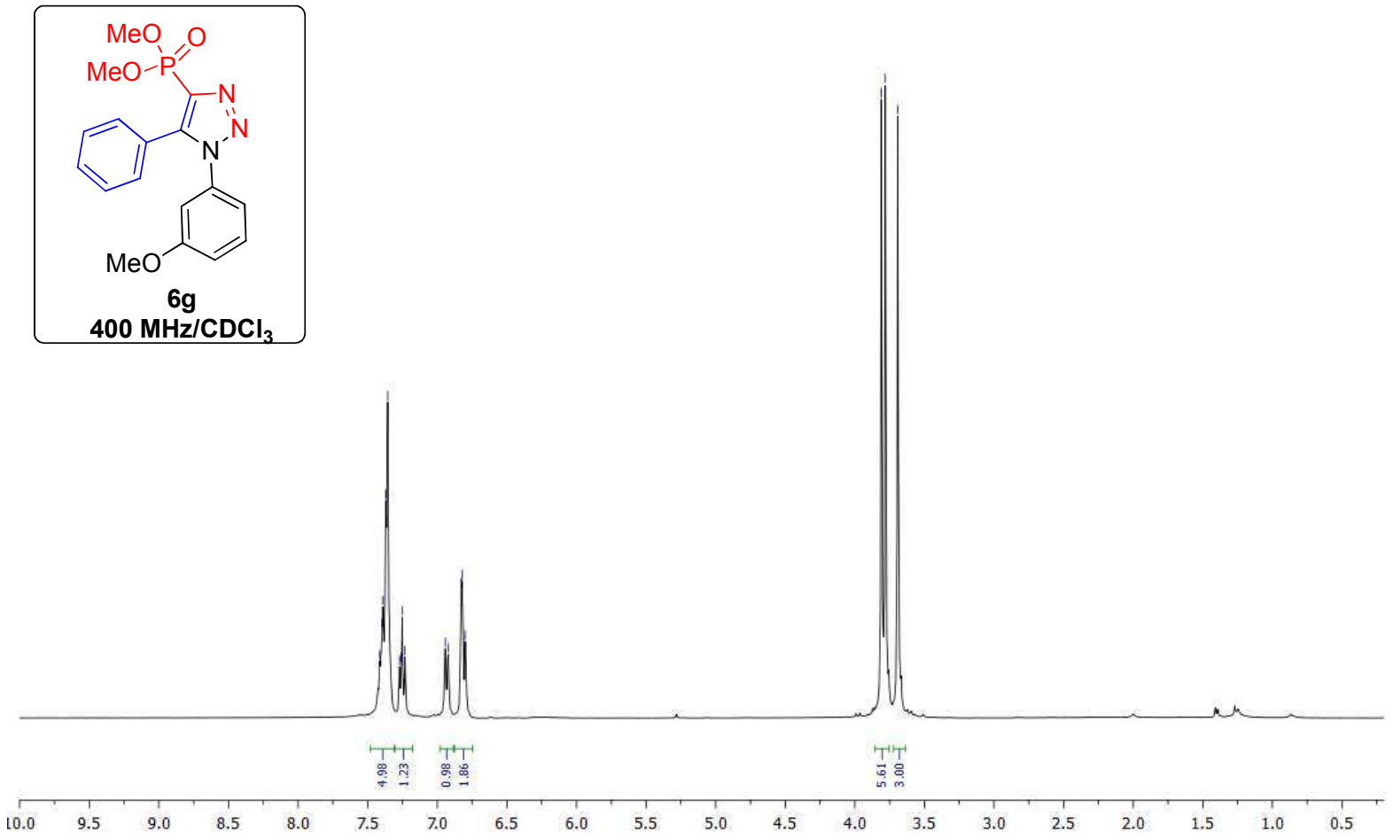

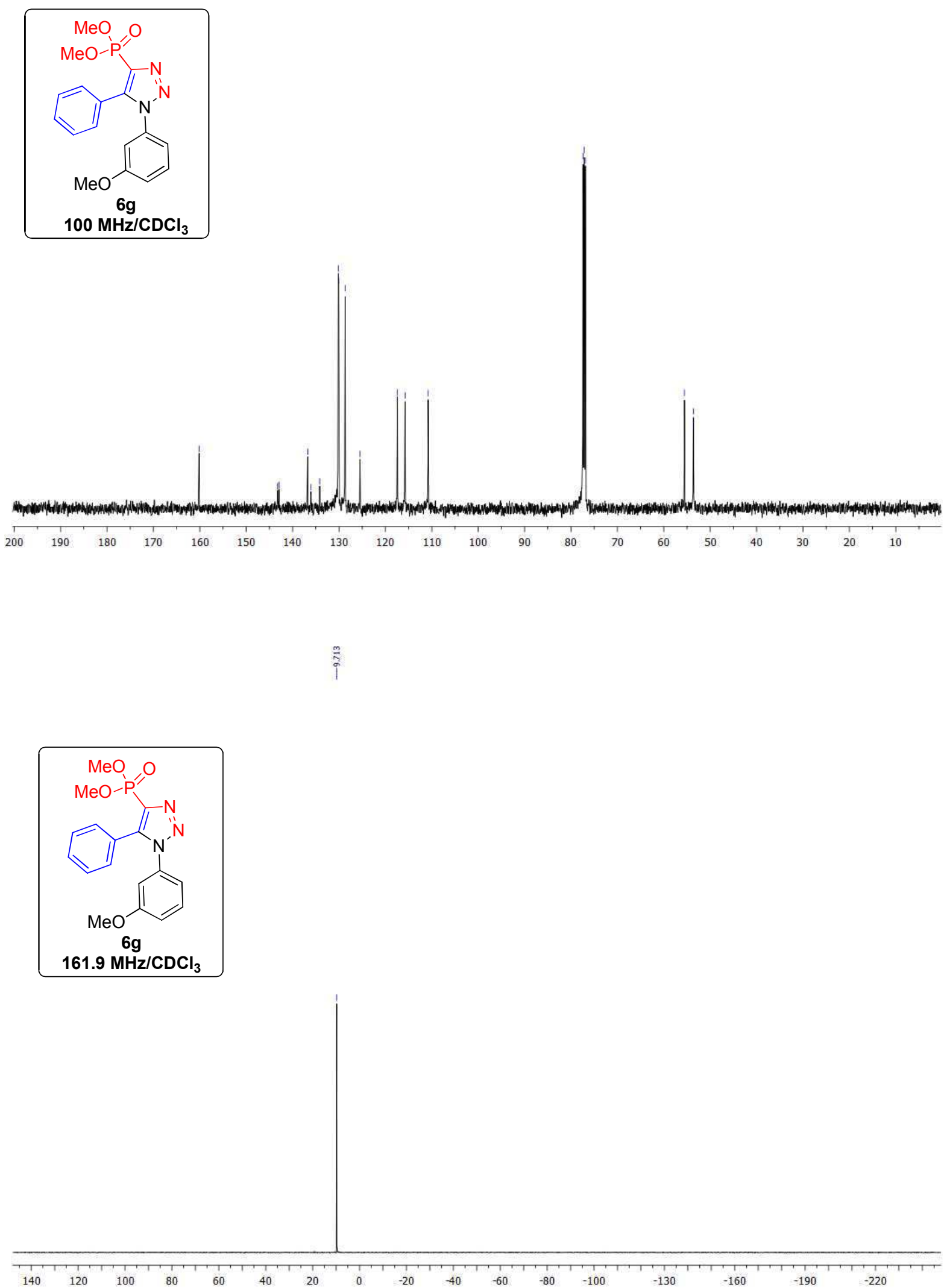

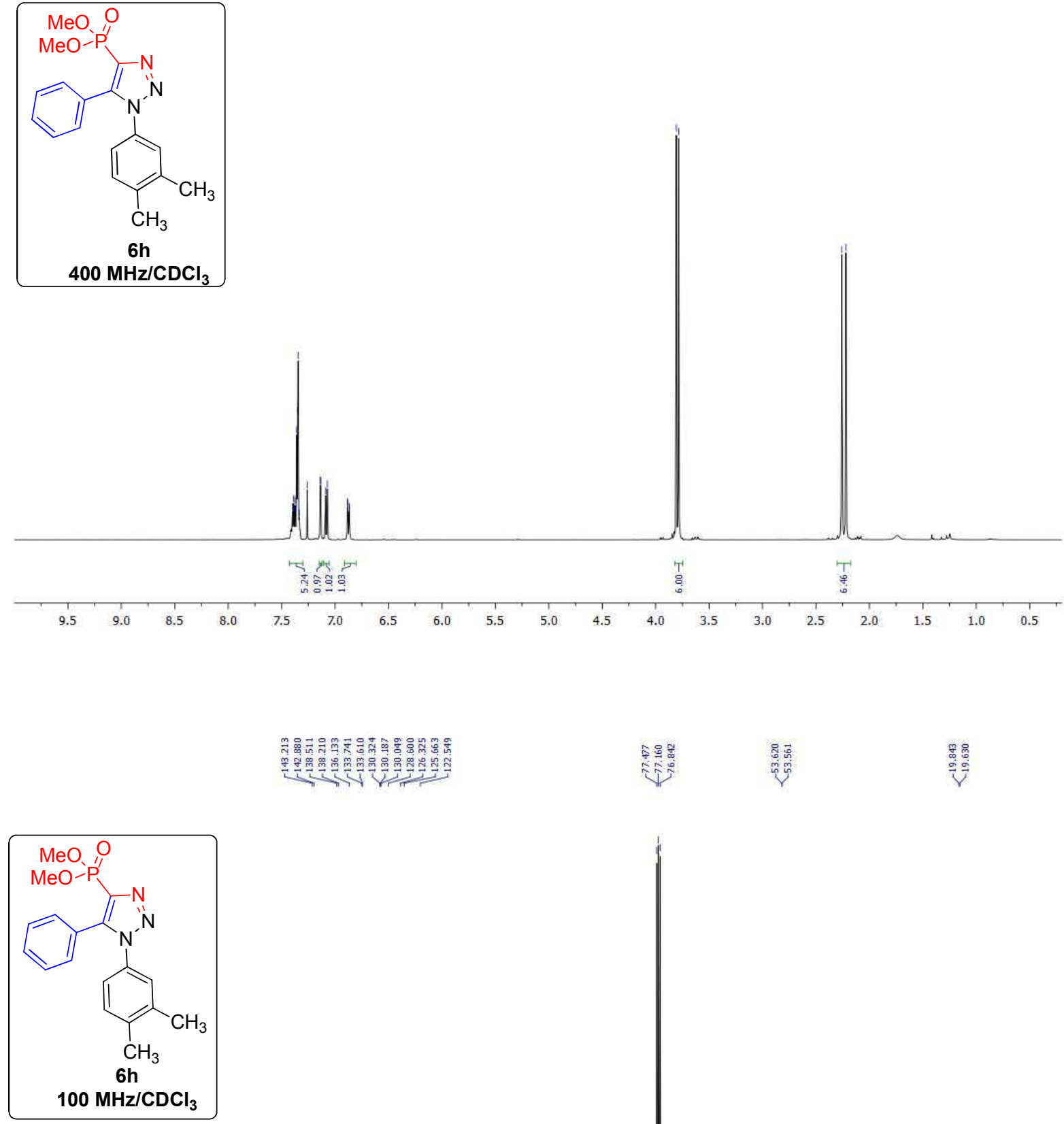

눈 눈

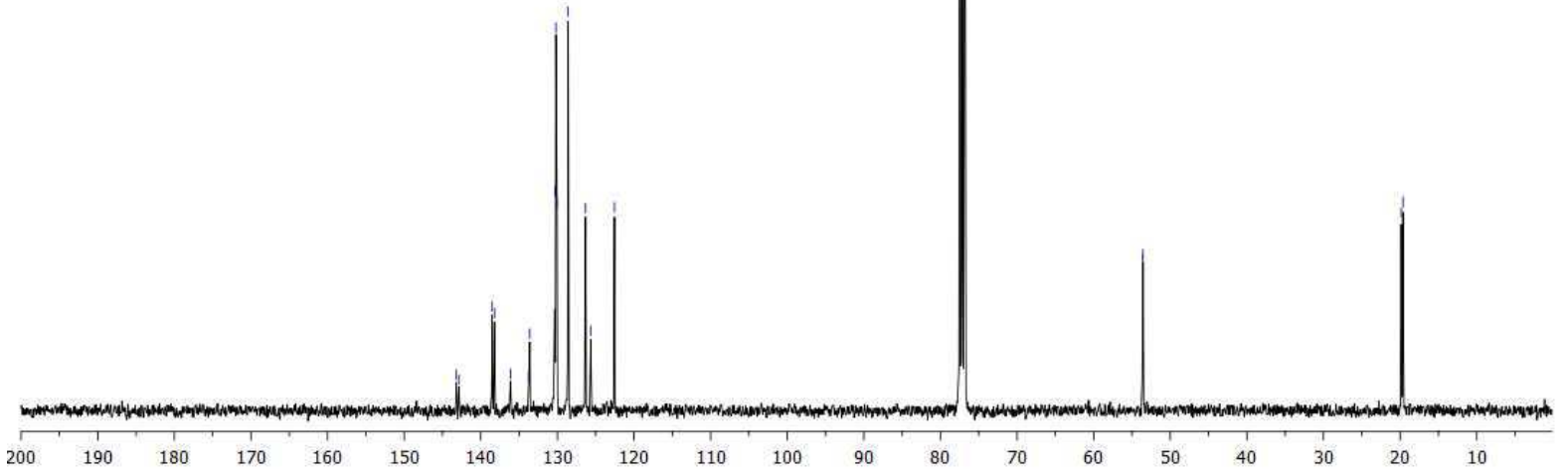



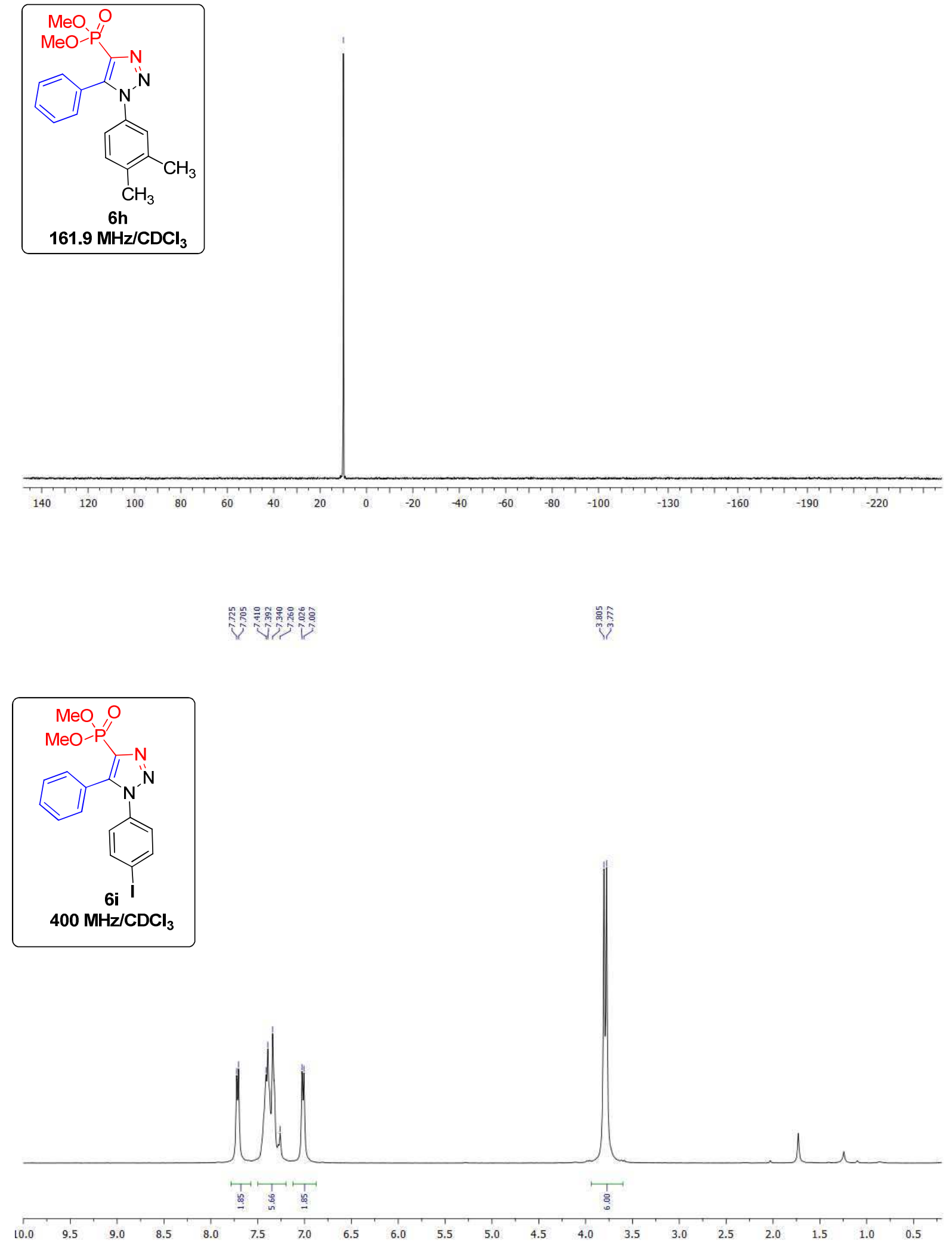


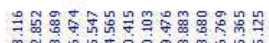

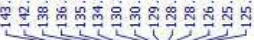

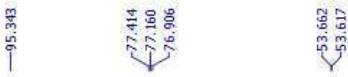
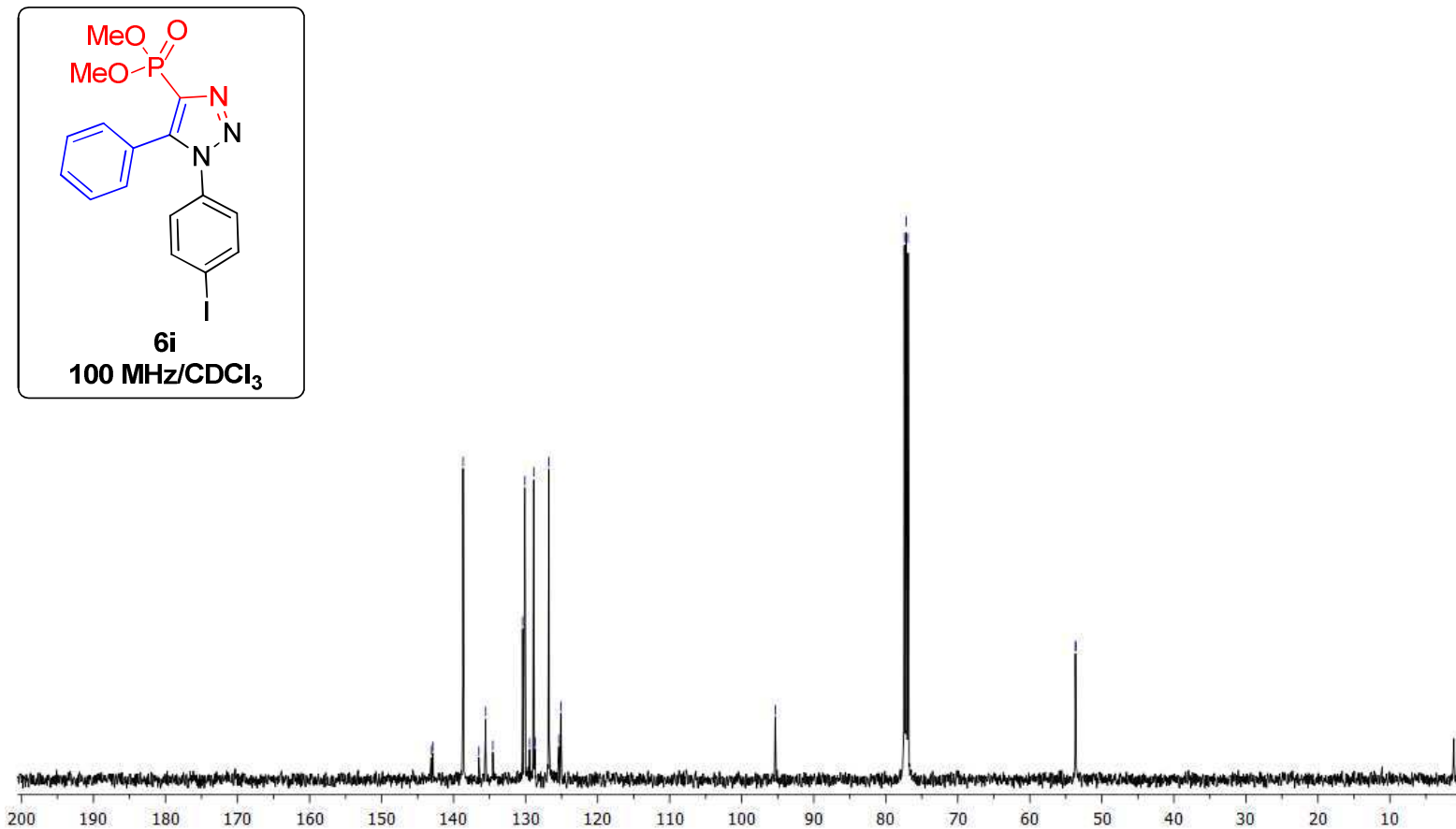

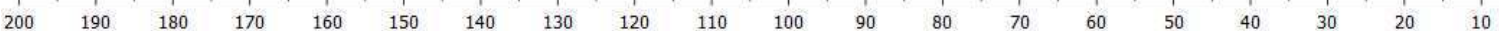
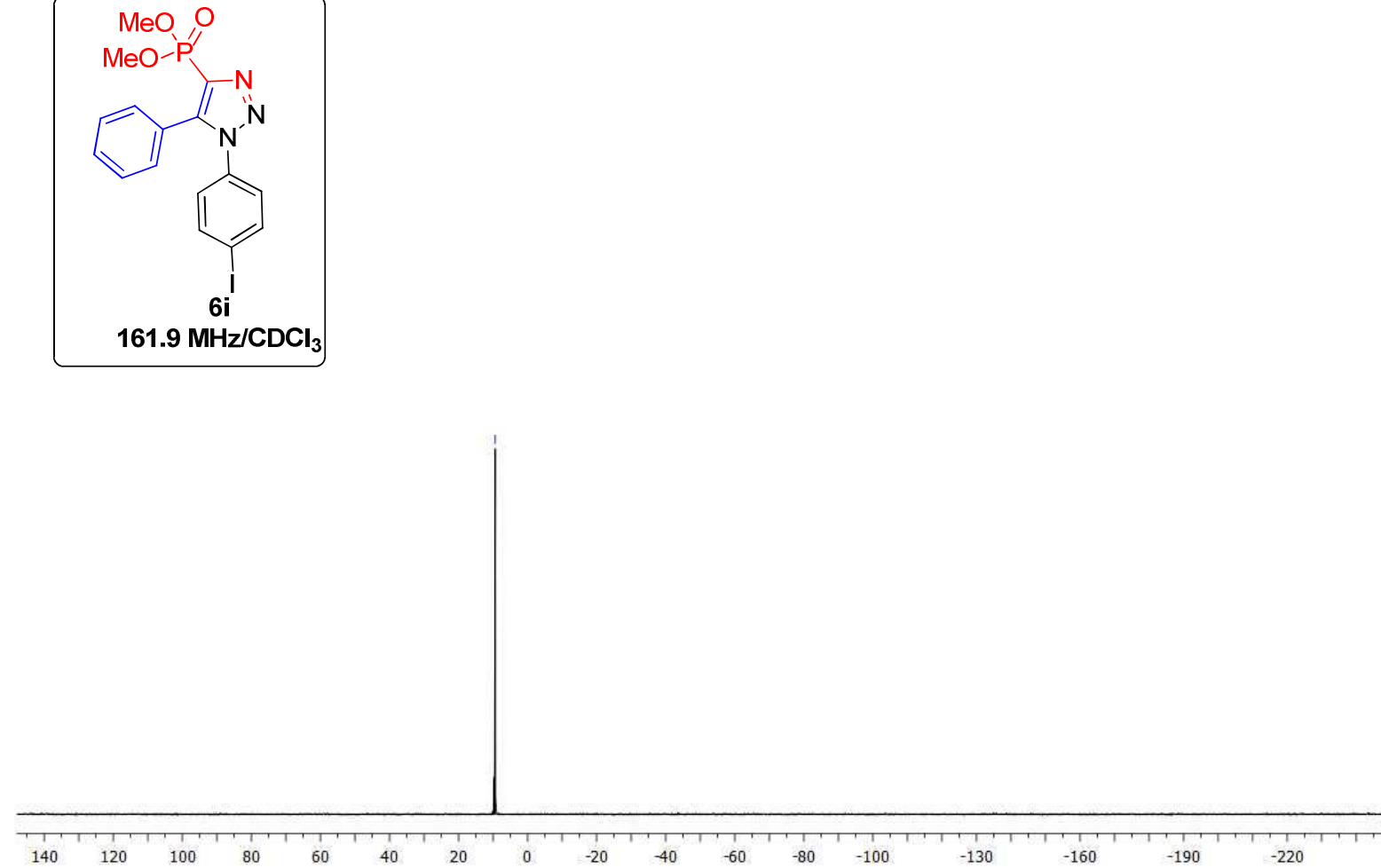

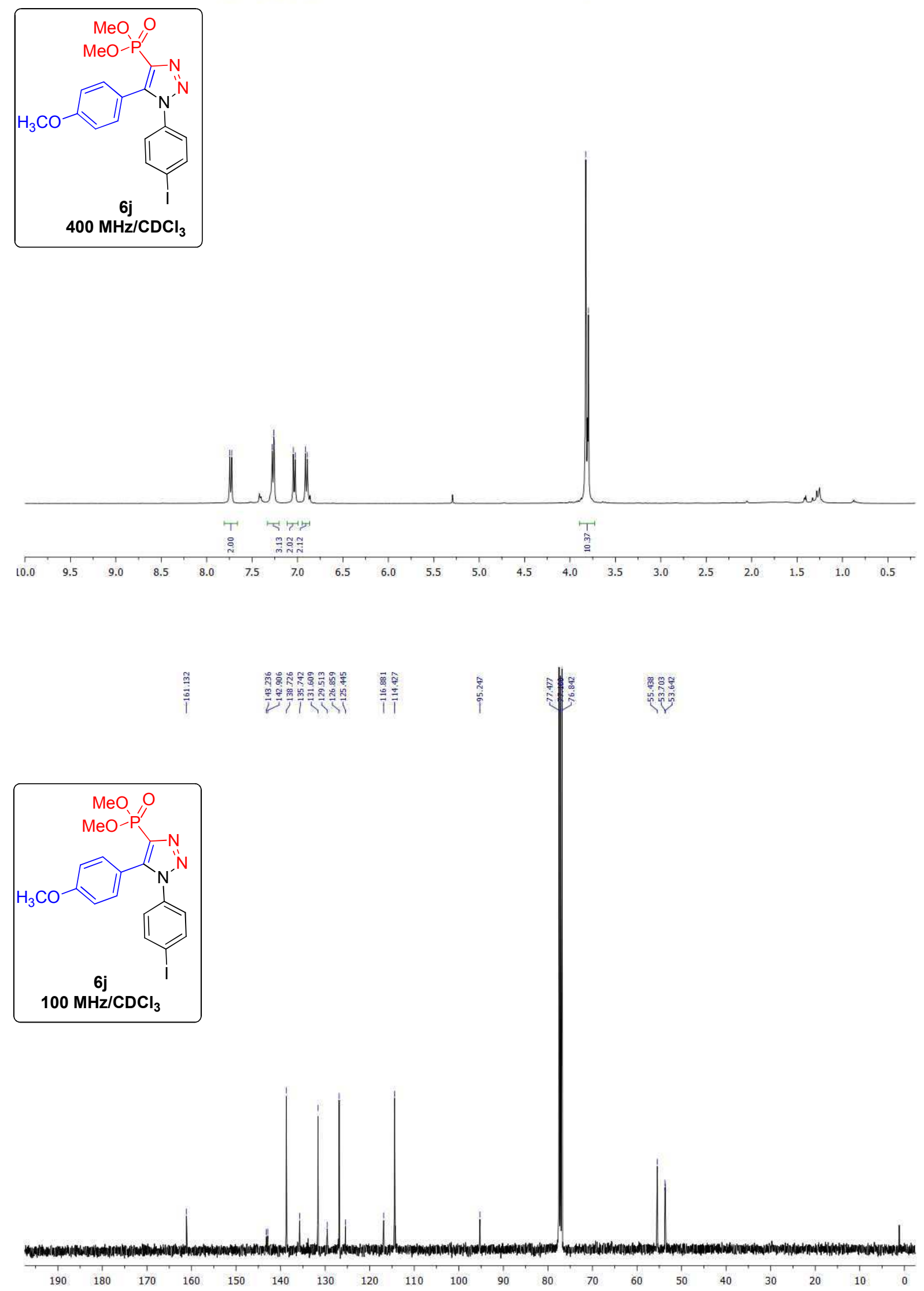

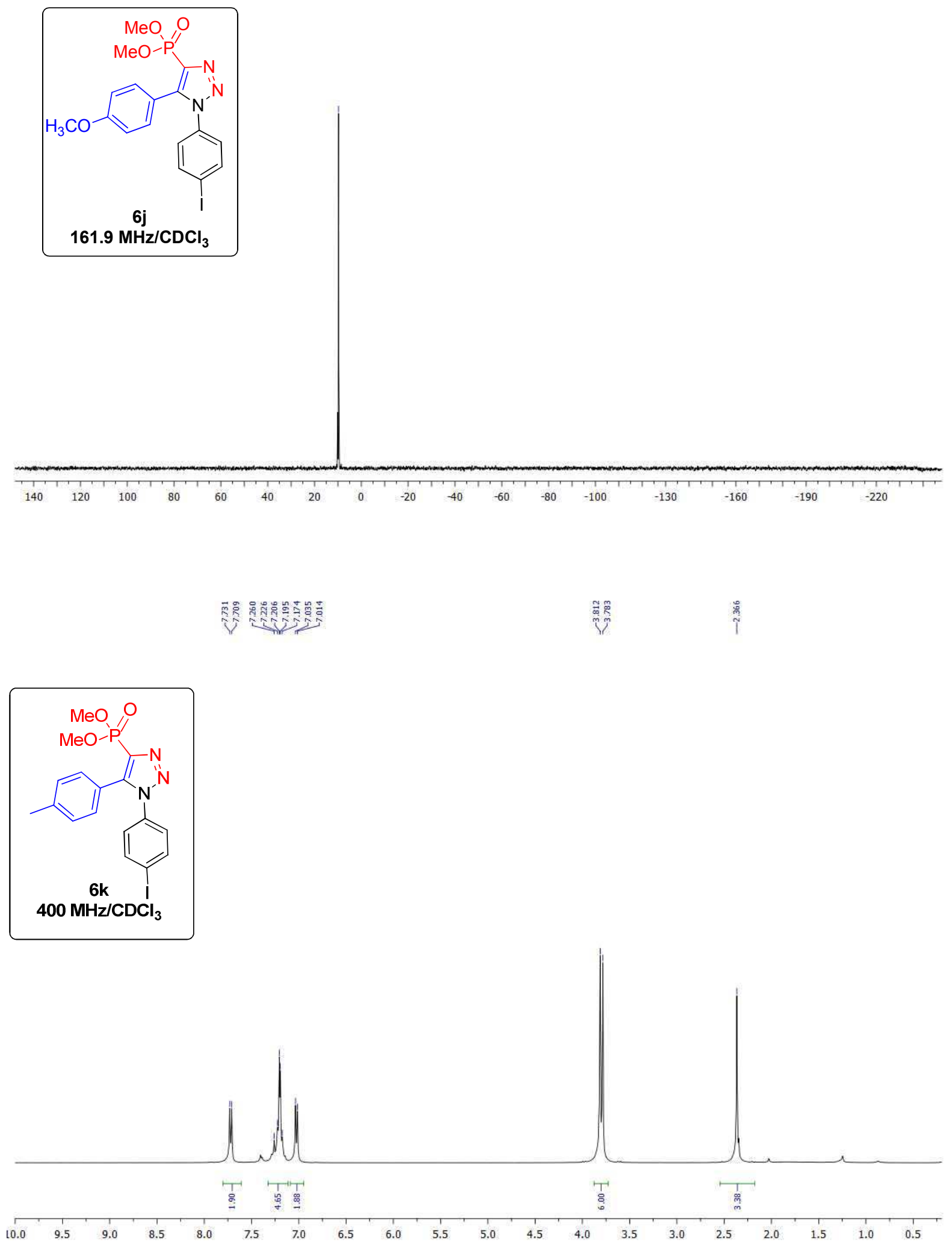


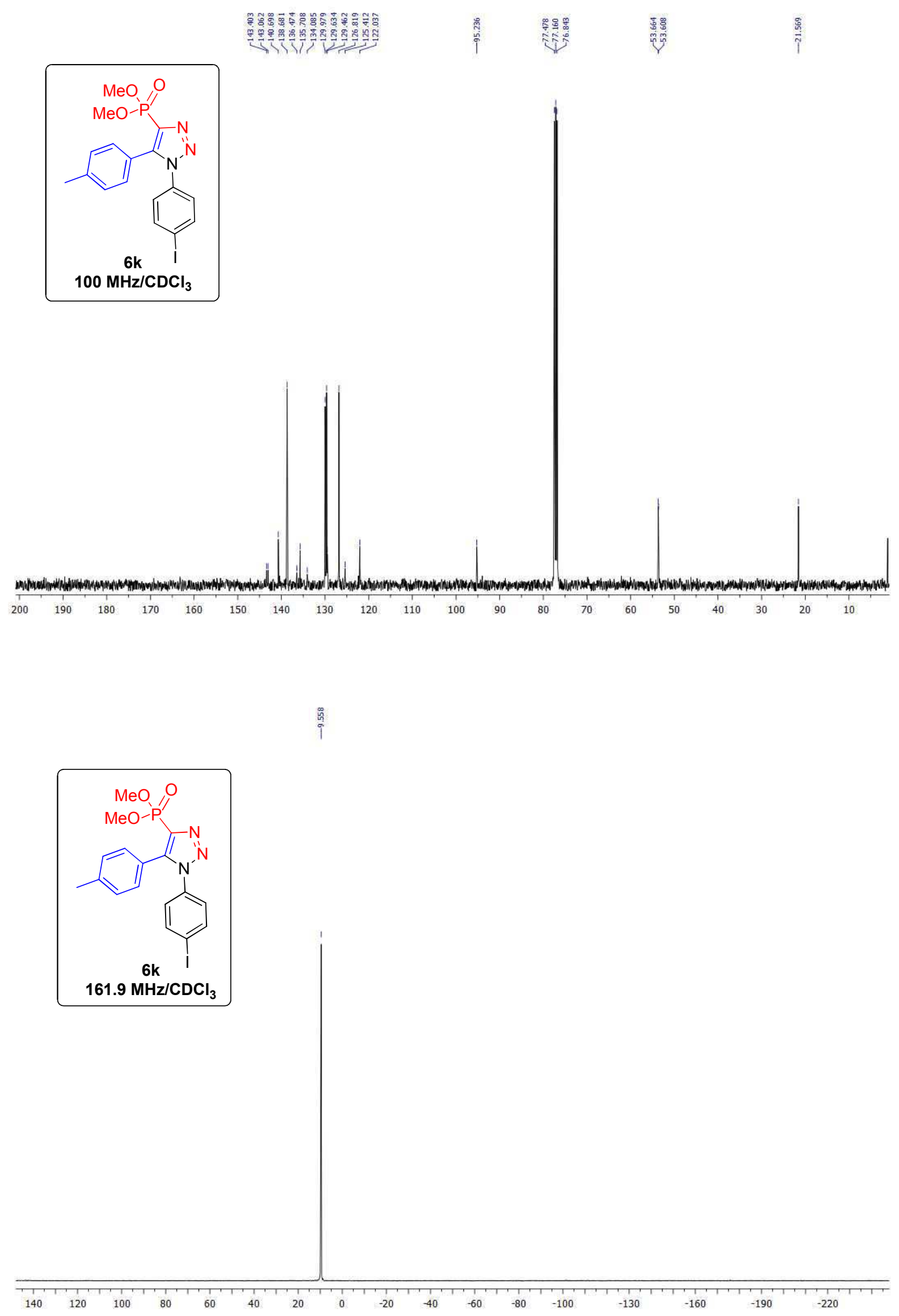



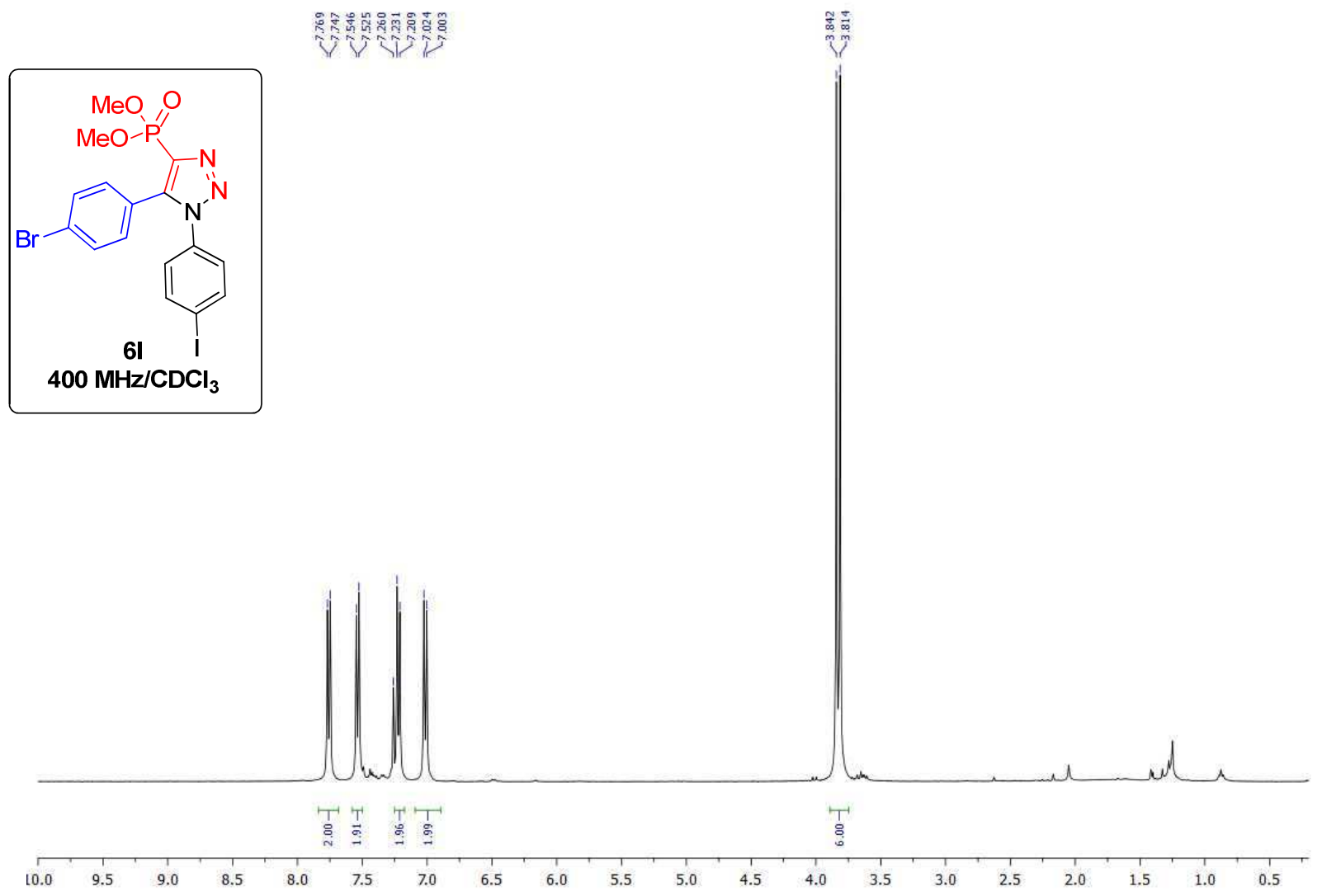

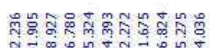

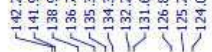
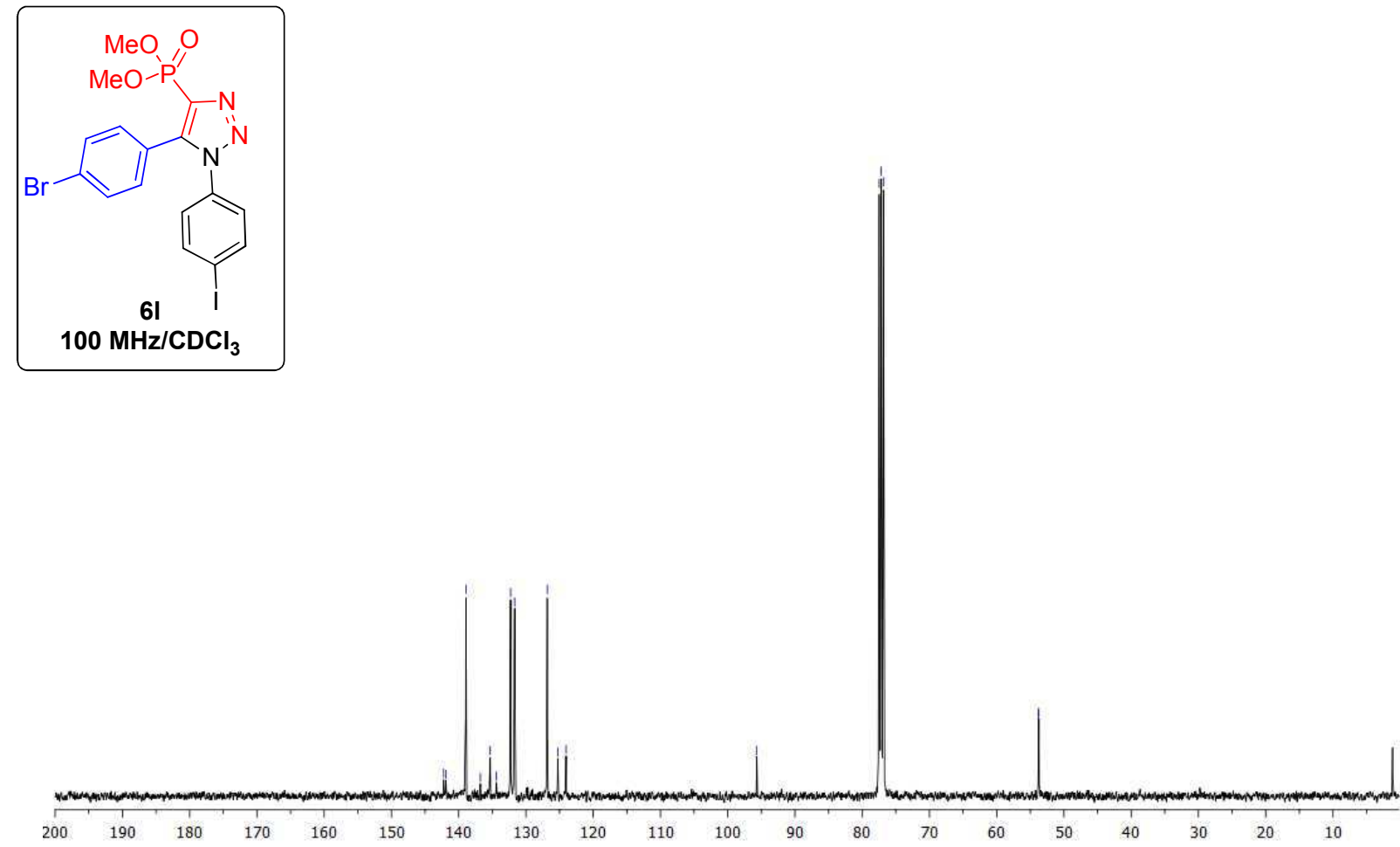


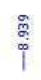
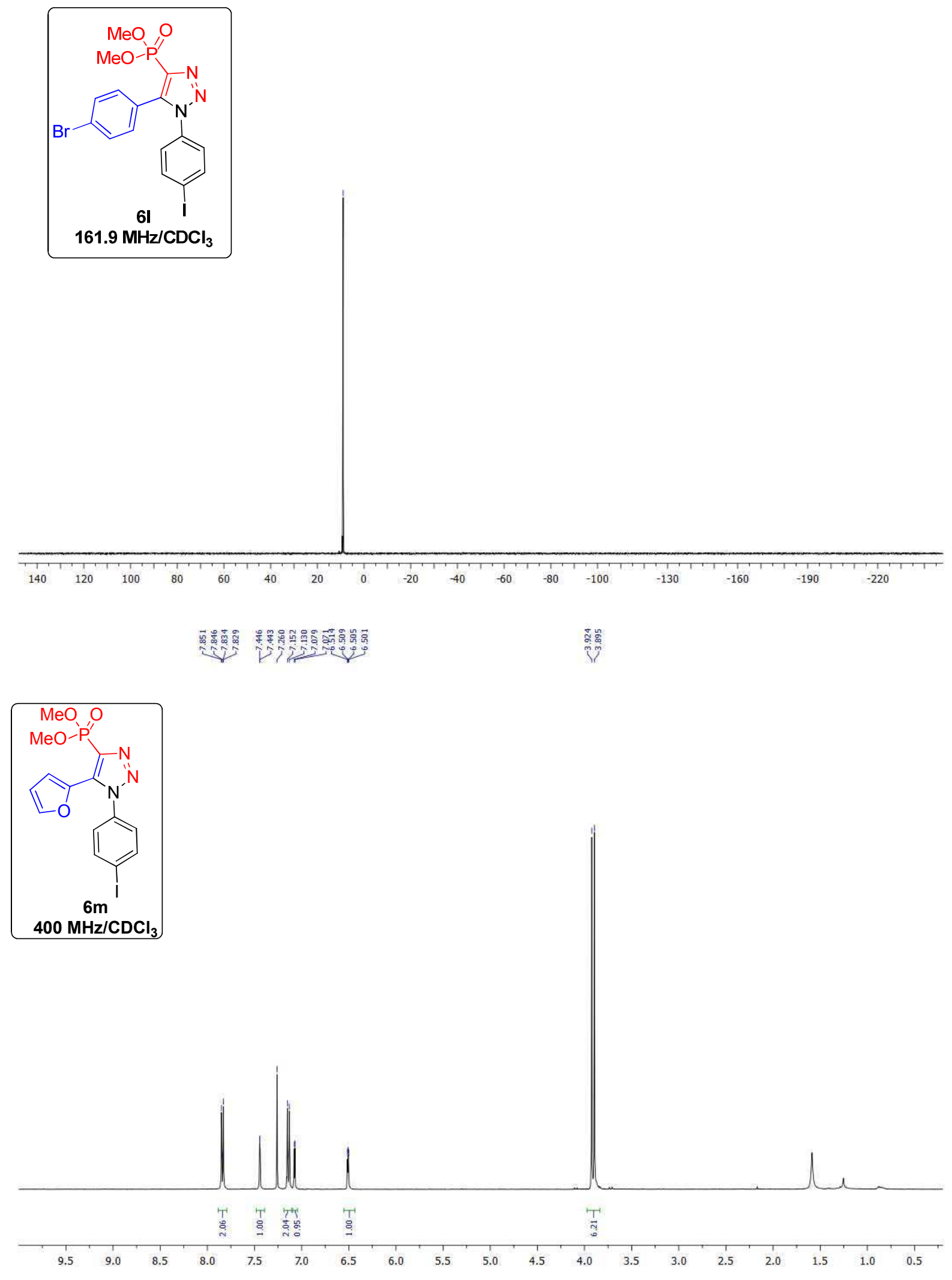

93 


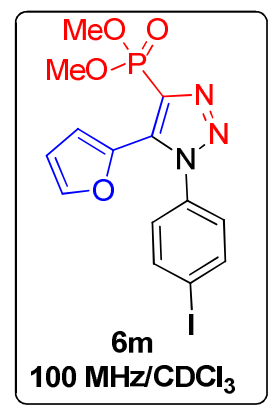

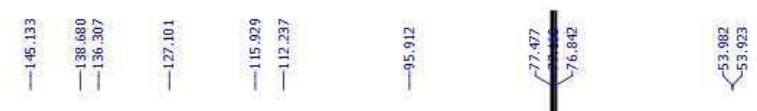

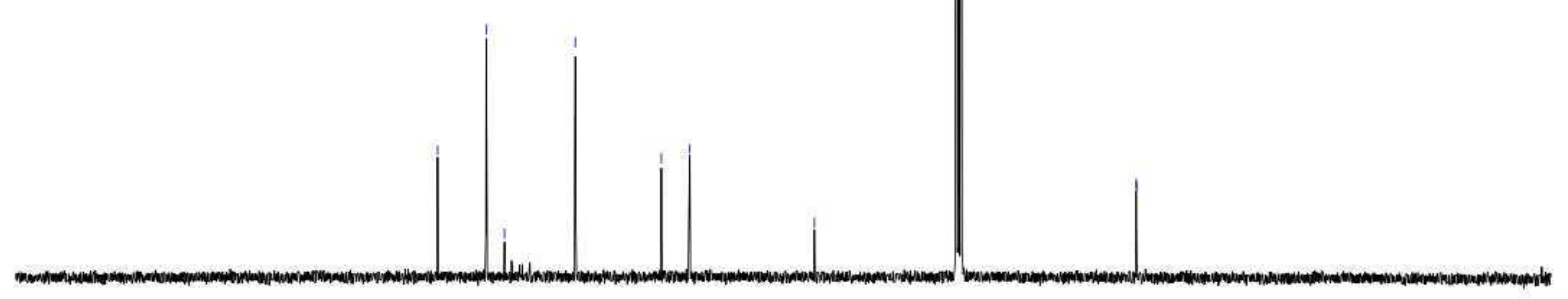

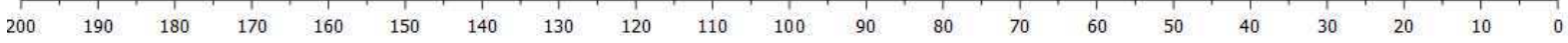

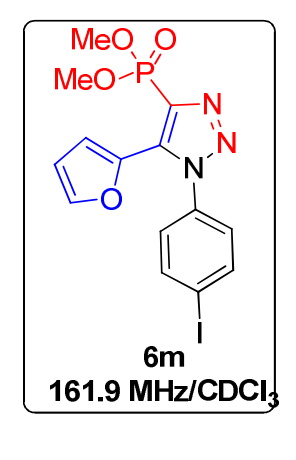

$\begin{array}{lllllllllllll}140 & 120 & 100 & 80 & 60 & 40 & 20 & 0 & -20 & -40 & -60 & -80 & -100\end{array}$

$-130$

$-160$ 


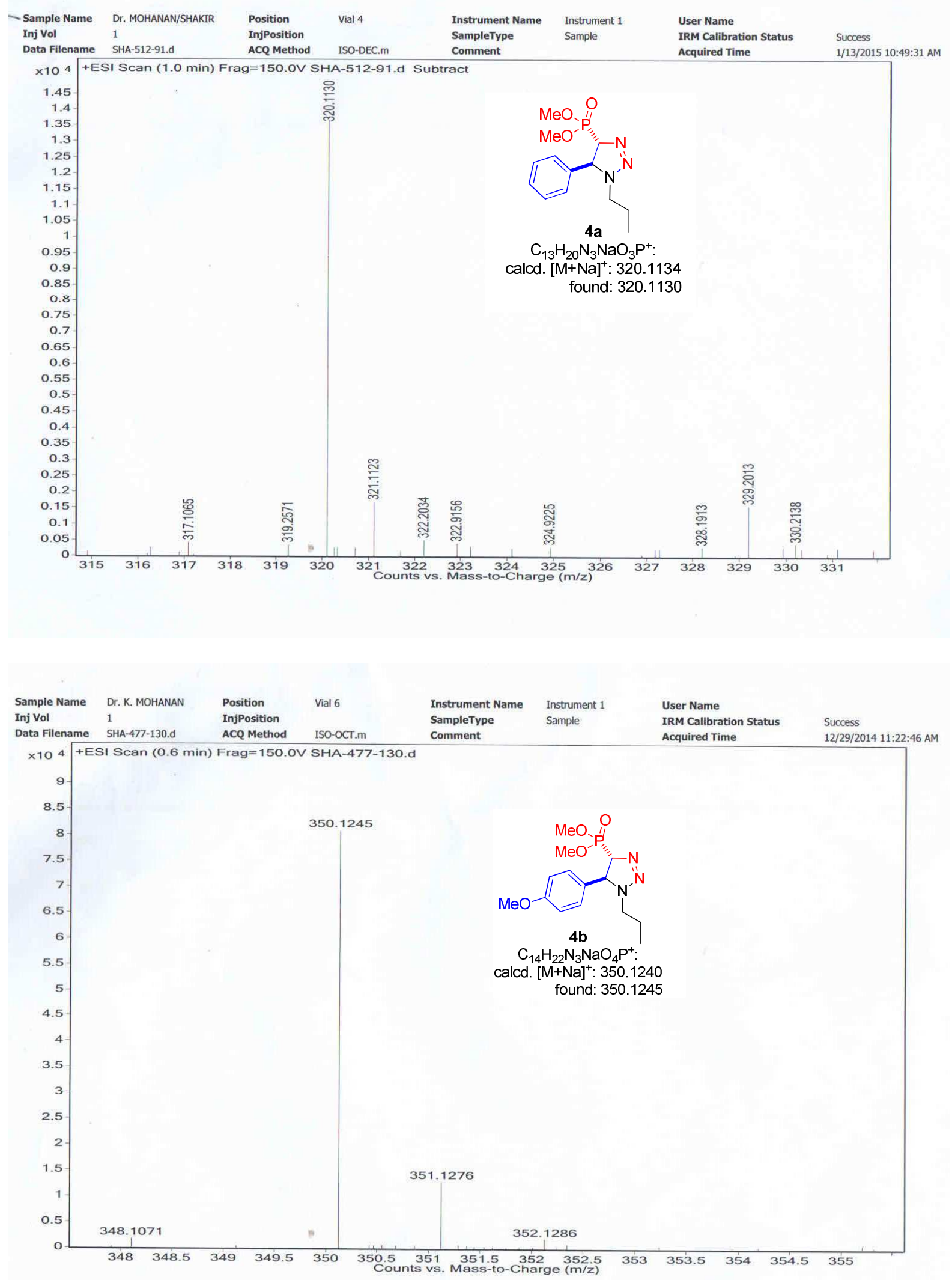




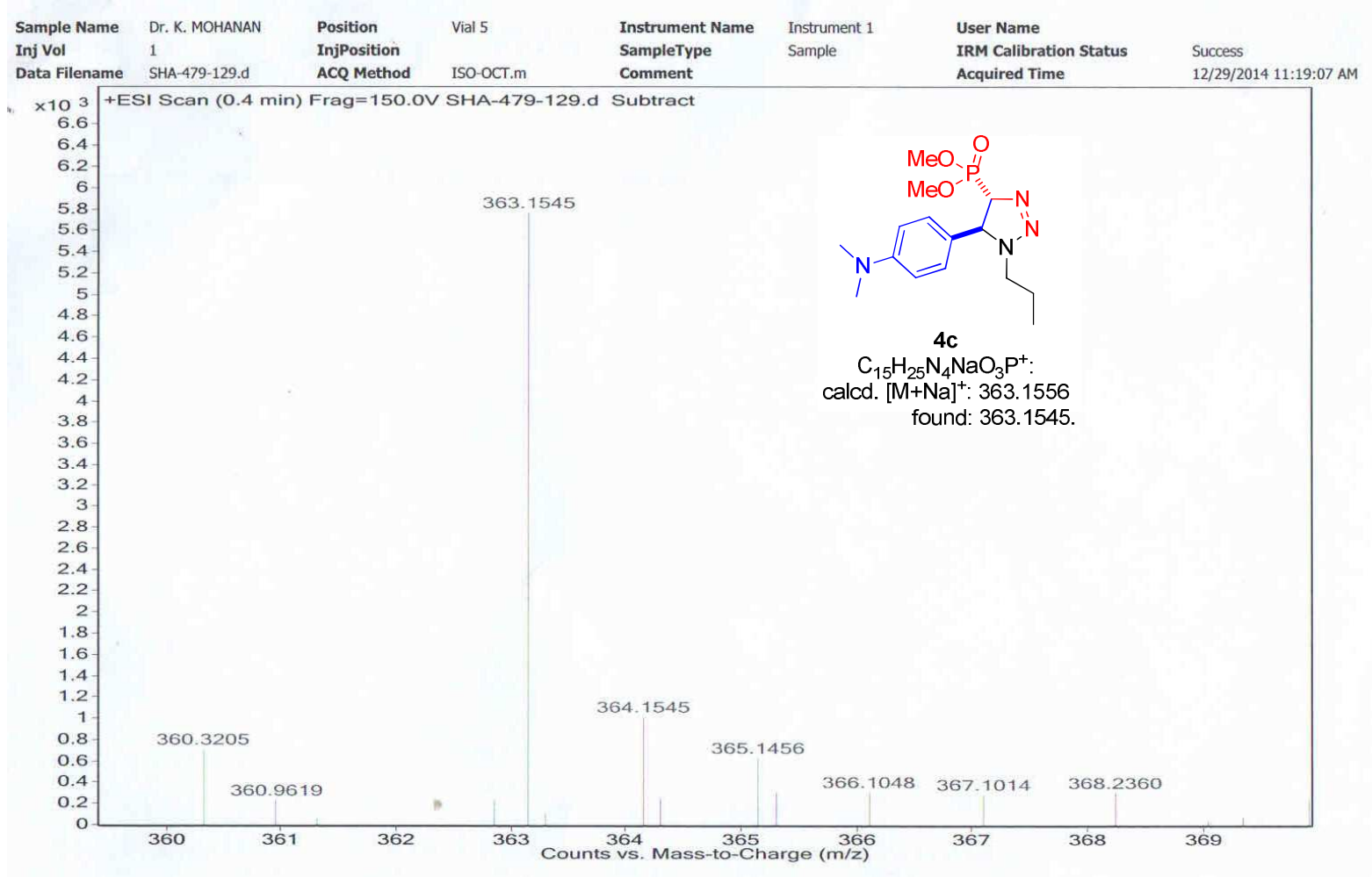

\begin{tabular}{|c|c|c|c|c|c|c|c|}
\hline Sample Name & Dr. MOHANAN/SHAKIR & Position & Vial 10 & Instrument Name & Instrument 1 & User Name & \\
\hline Inj Vol & 1 & InjPosition & & SampleType & Sample & IRM Calibration Status & Success \\
\hline Data Filename & SHA-589_080.d & ACQ Method & ISO-DEC.m & Comment & & Acquired Time & 5/14/2015 10:52:56 AM \\
\hline
\end{tabular}
$\times 10^{4}+$ ESI Scan (0.6 min) Frag=150.0V SHA-589_080.d Subtract

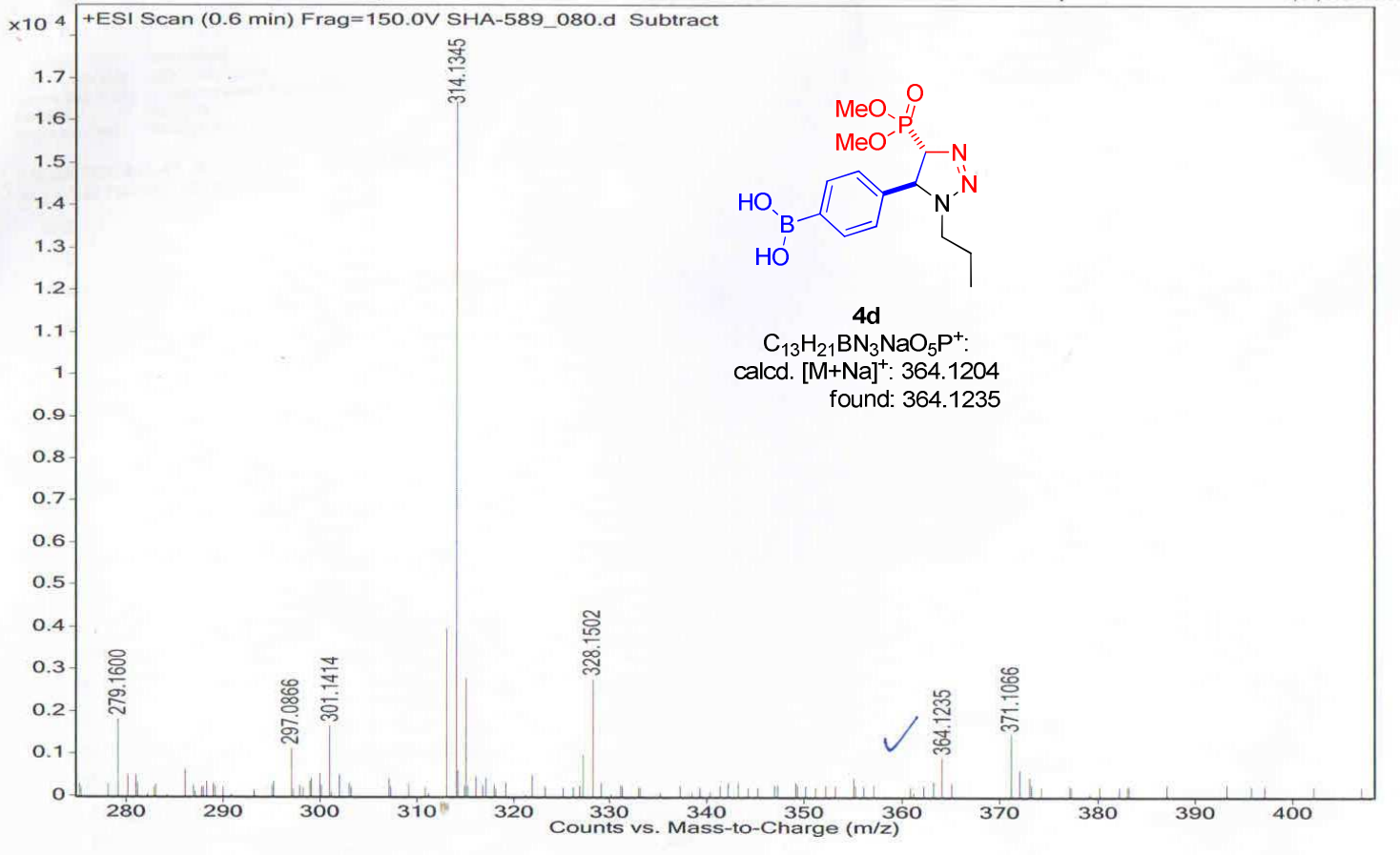



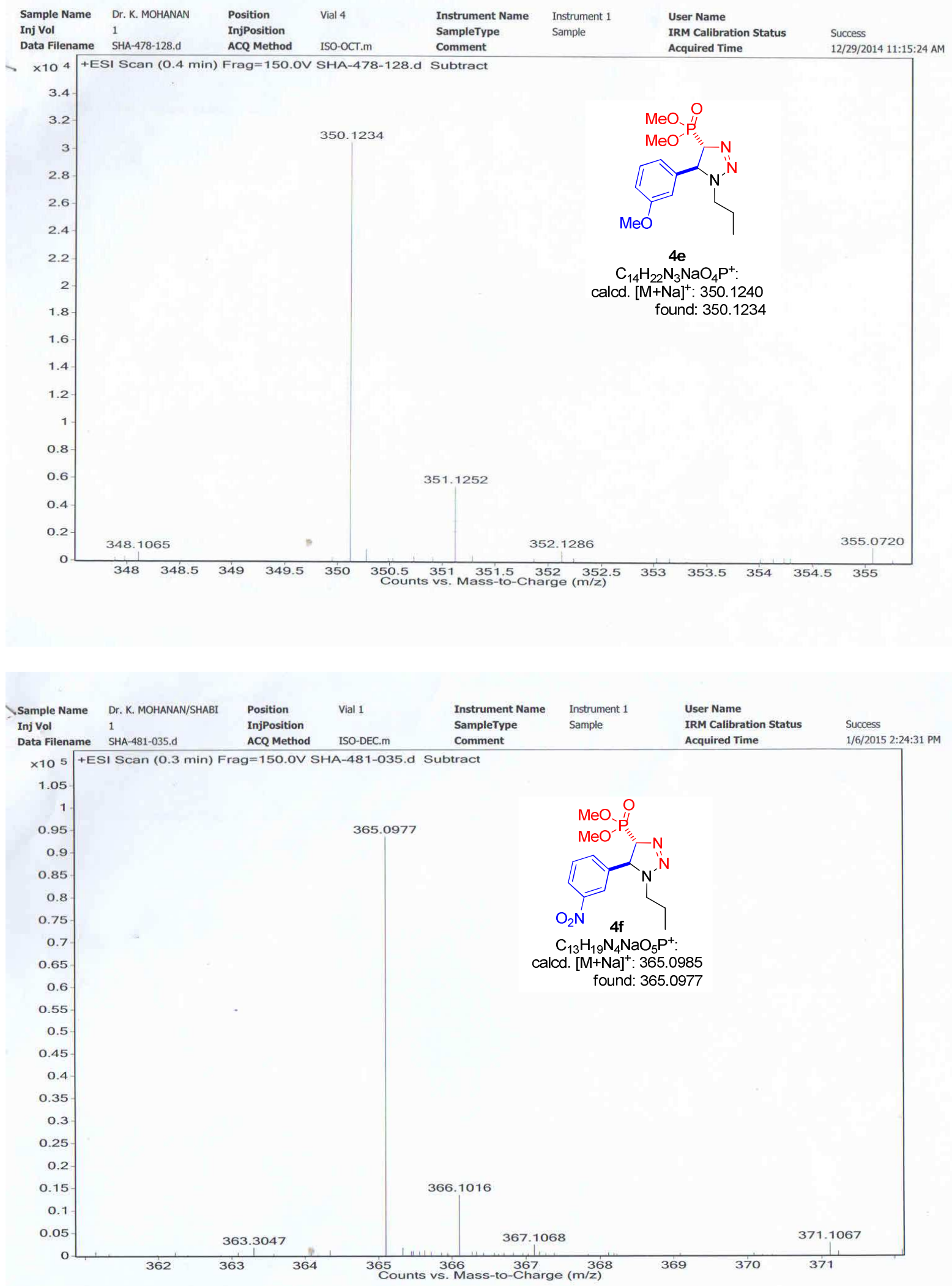


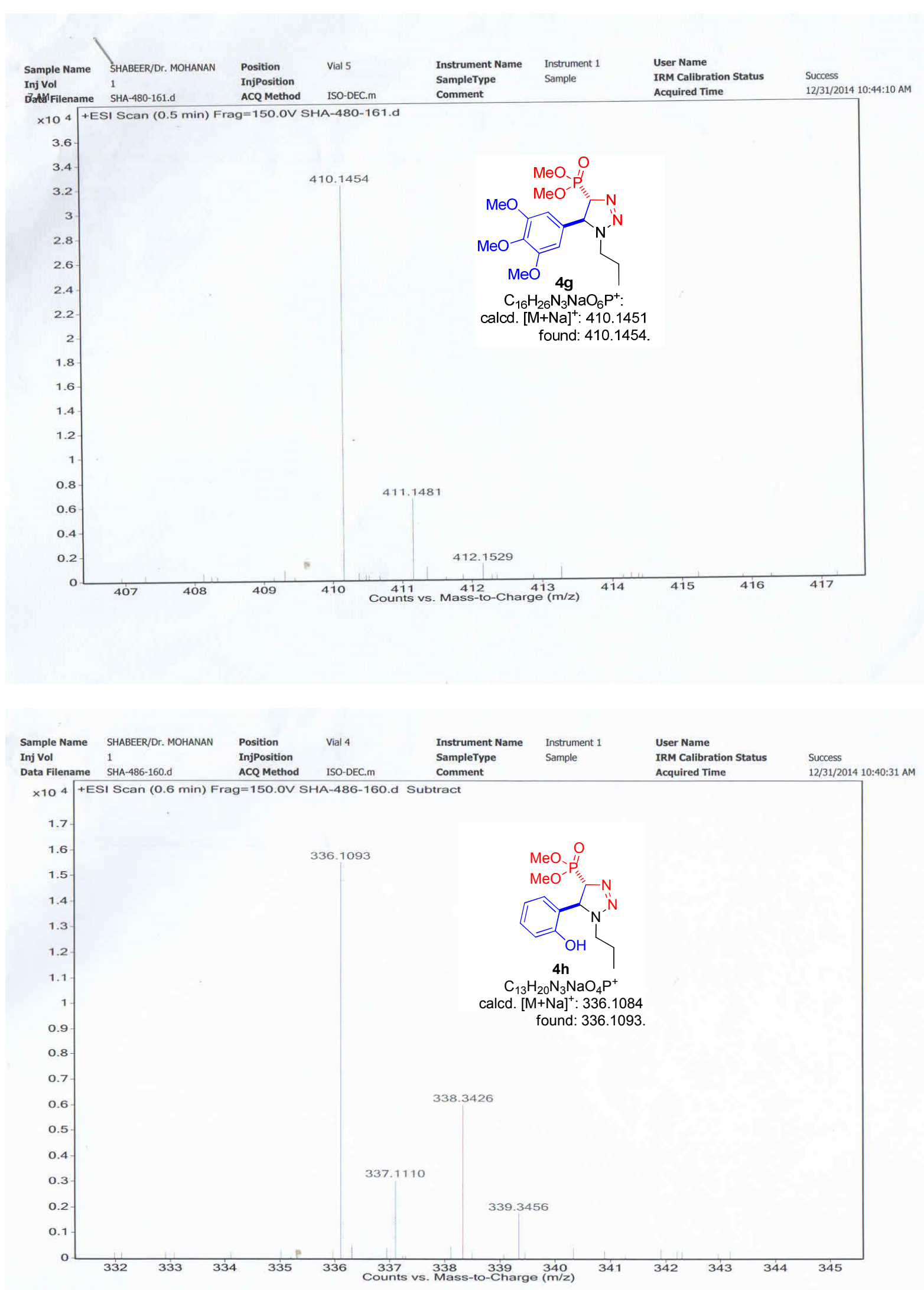



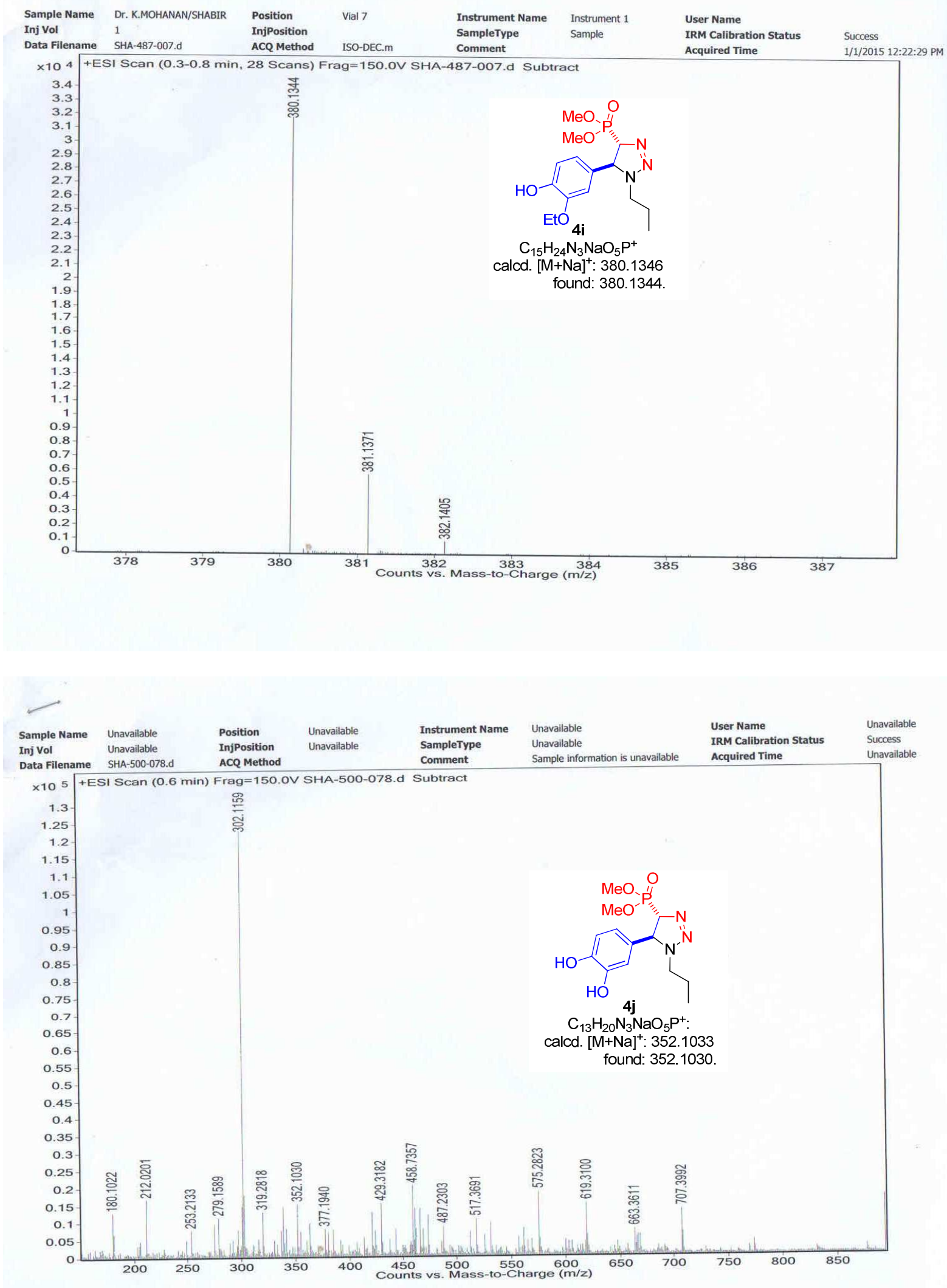
4 SHA-482\#11 RT: 0.16 AV: 1 NL: $1.52 E 7$

T: FTMS + c ESI Full ms [100.00-2000.00]
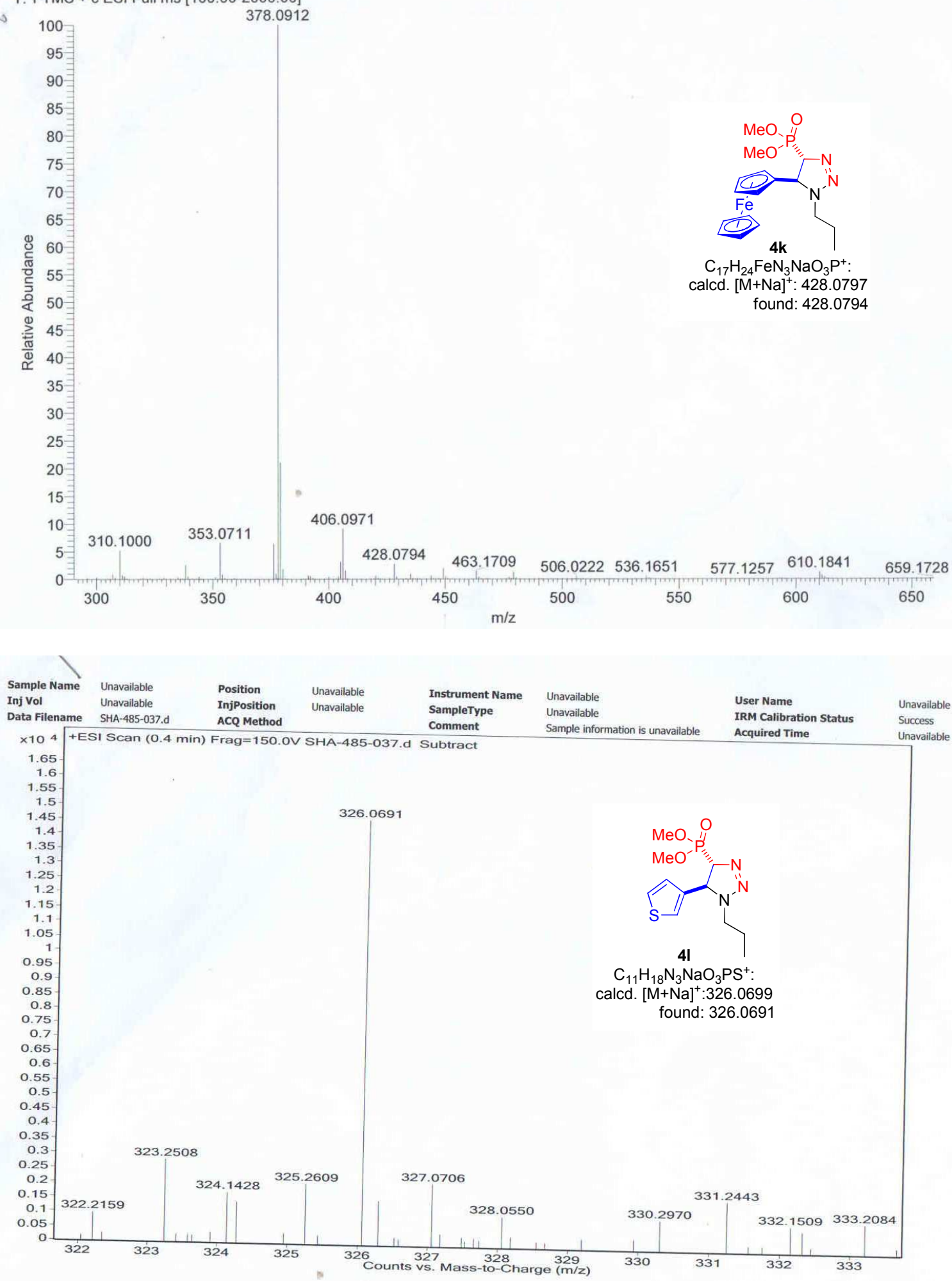

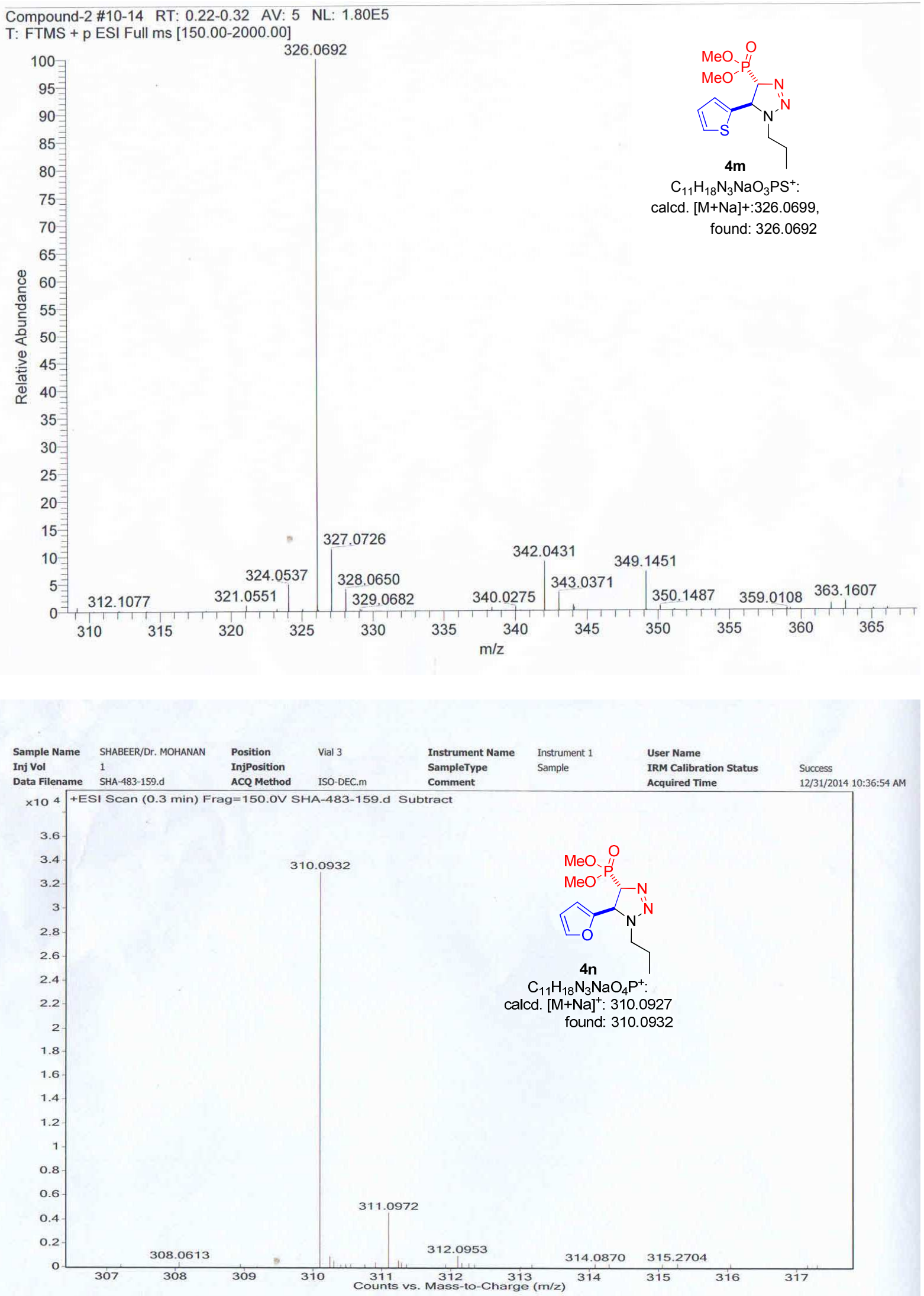

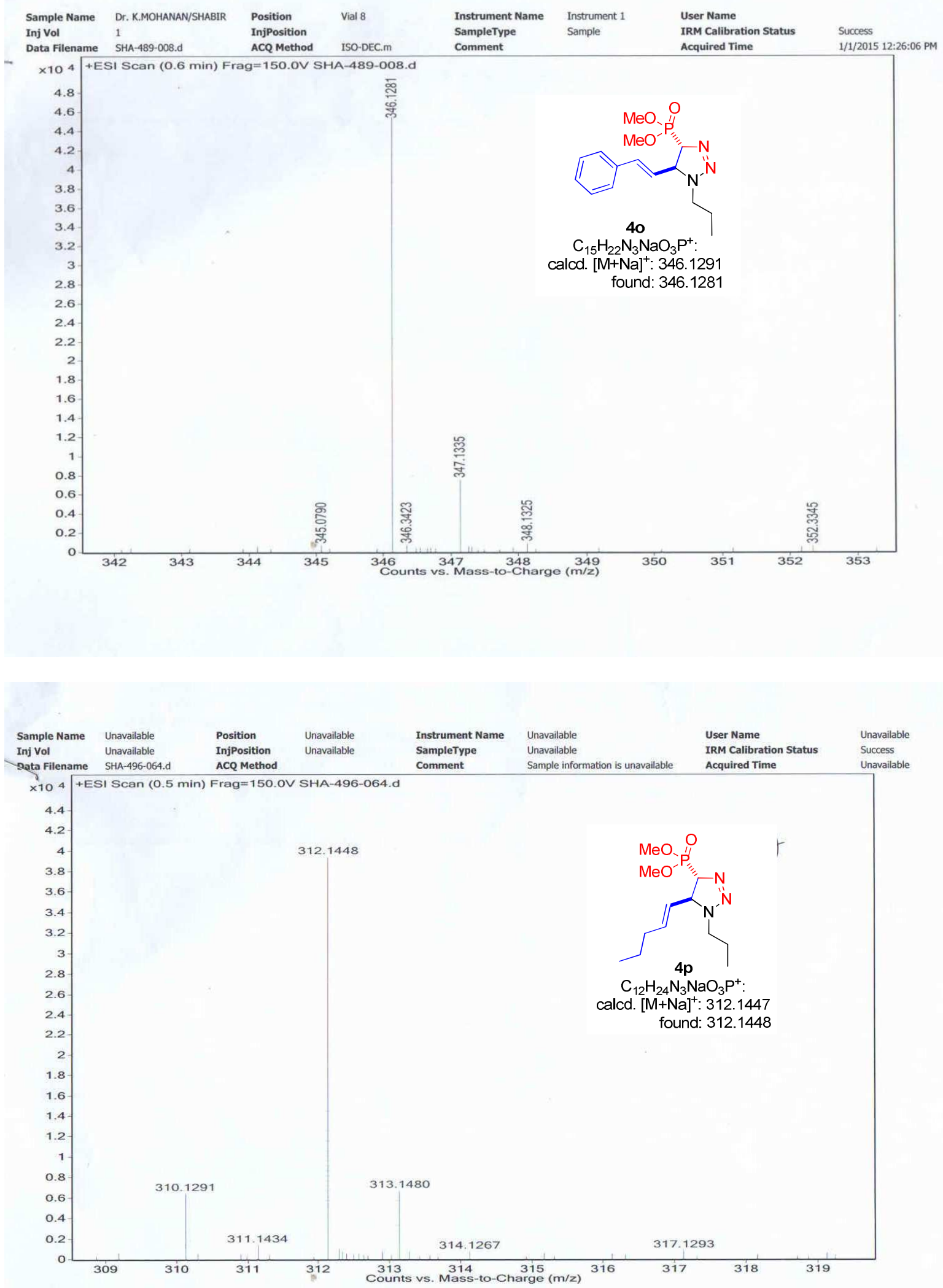

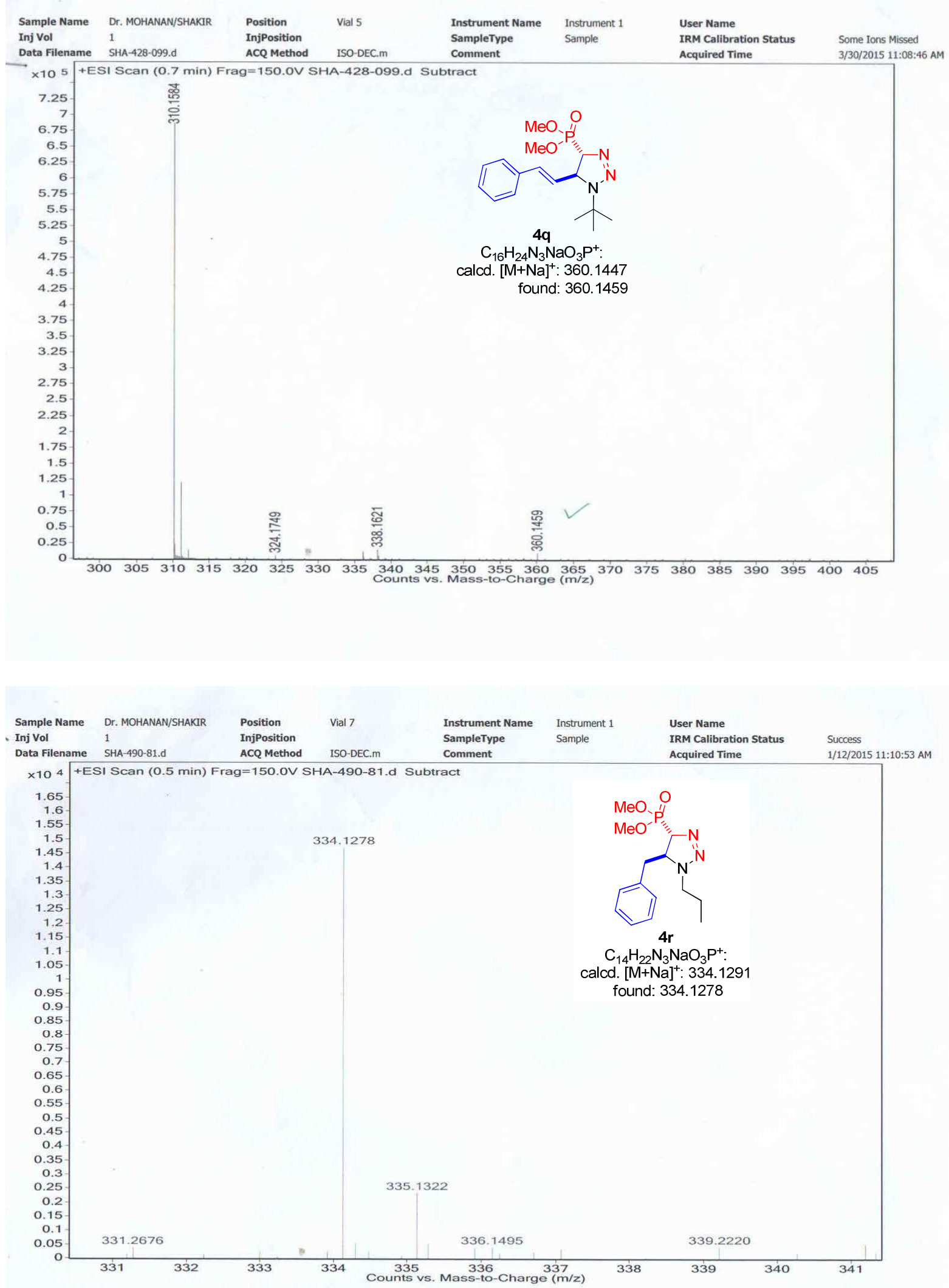


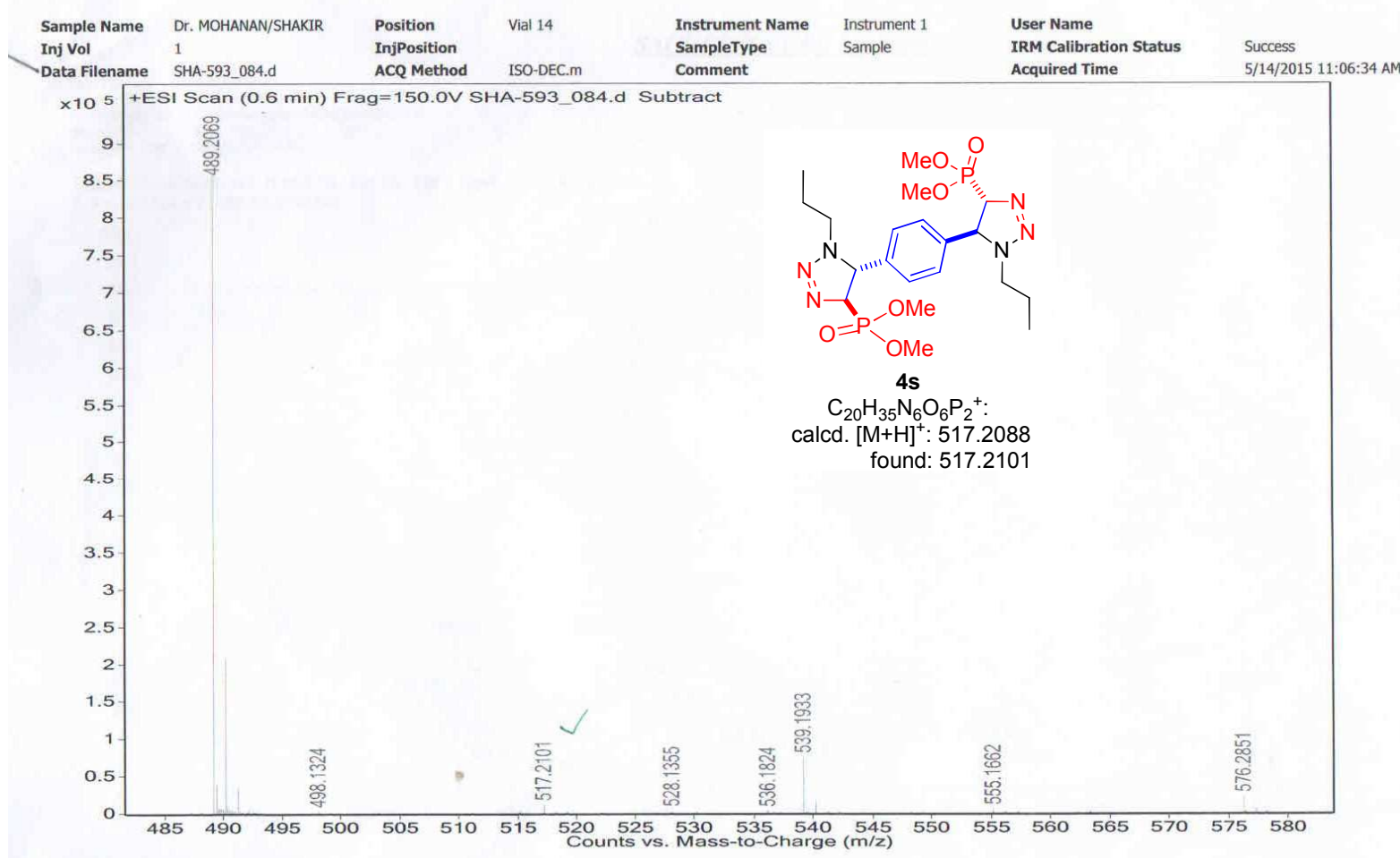

D:INTERNALSI...ISHA-447_150323122743

3/23/2015 12:27:43 PM

Dr. MOHANAN/SHAKIR

SHA-447 150323122743 \#11 RT: 0.15 AV: 1 SB: 4 0.01-0.05 NL: 6.38E6

T: FTMS + c ESI Full ms [100.00-2000.00]

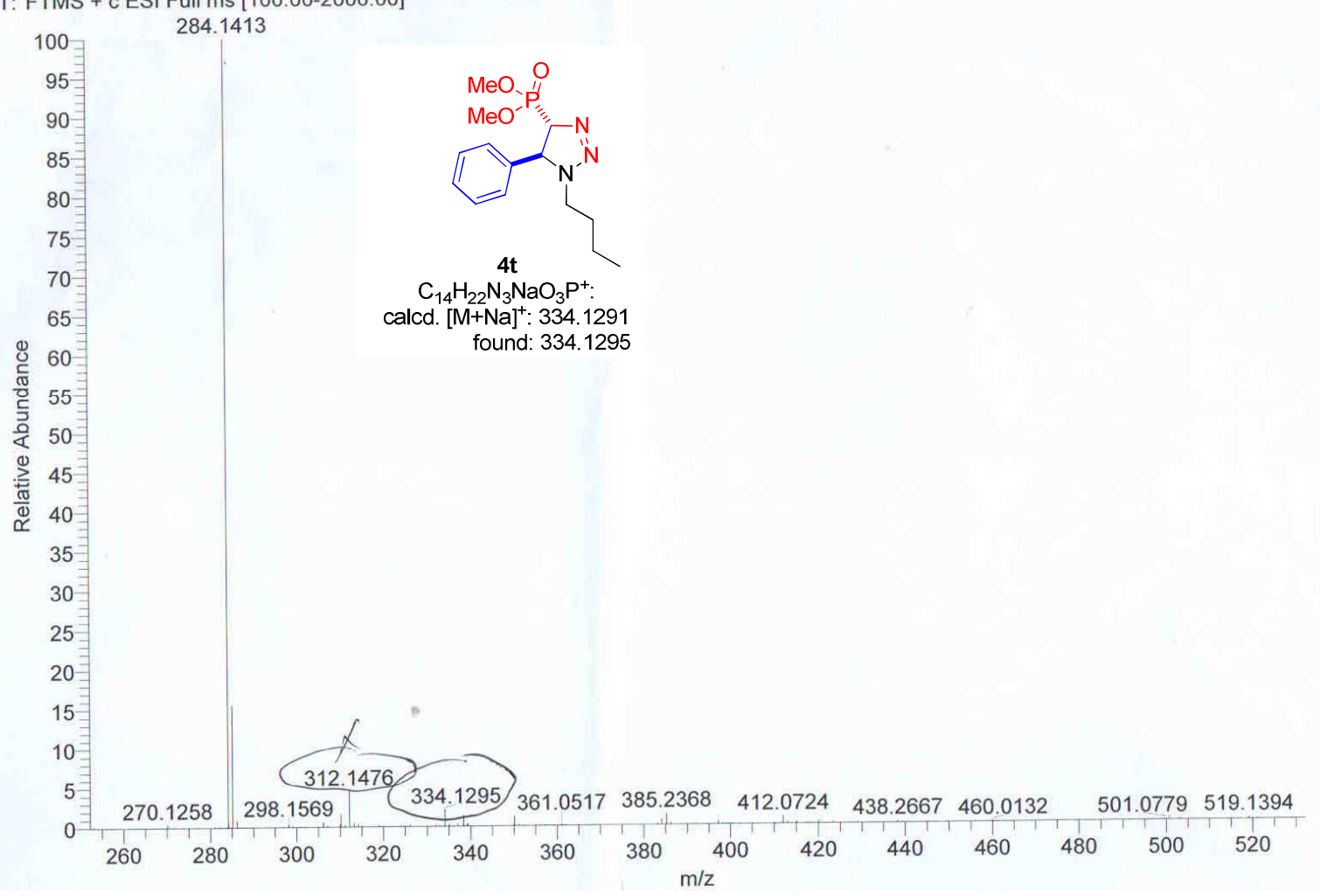


- SHA-503 \#22 RT: 0.19 AV: 1 NL: $1.52 E 6$

T: FTMS + c ESI Full ms [100.00-2000.00]
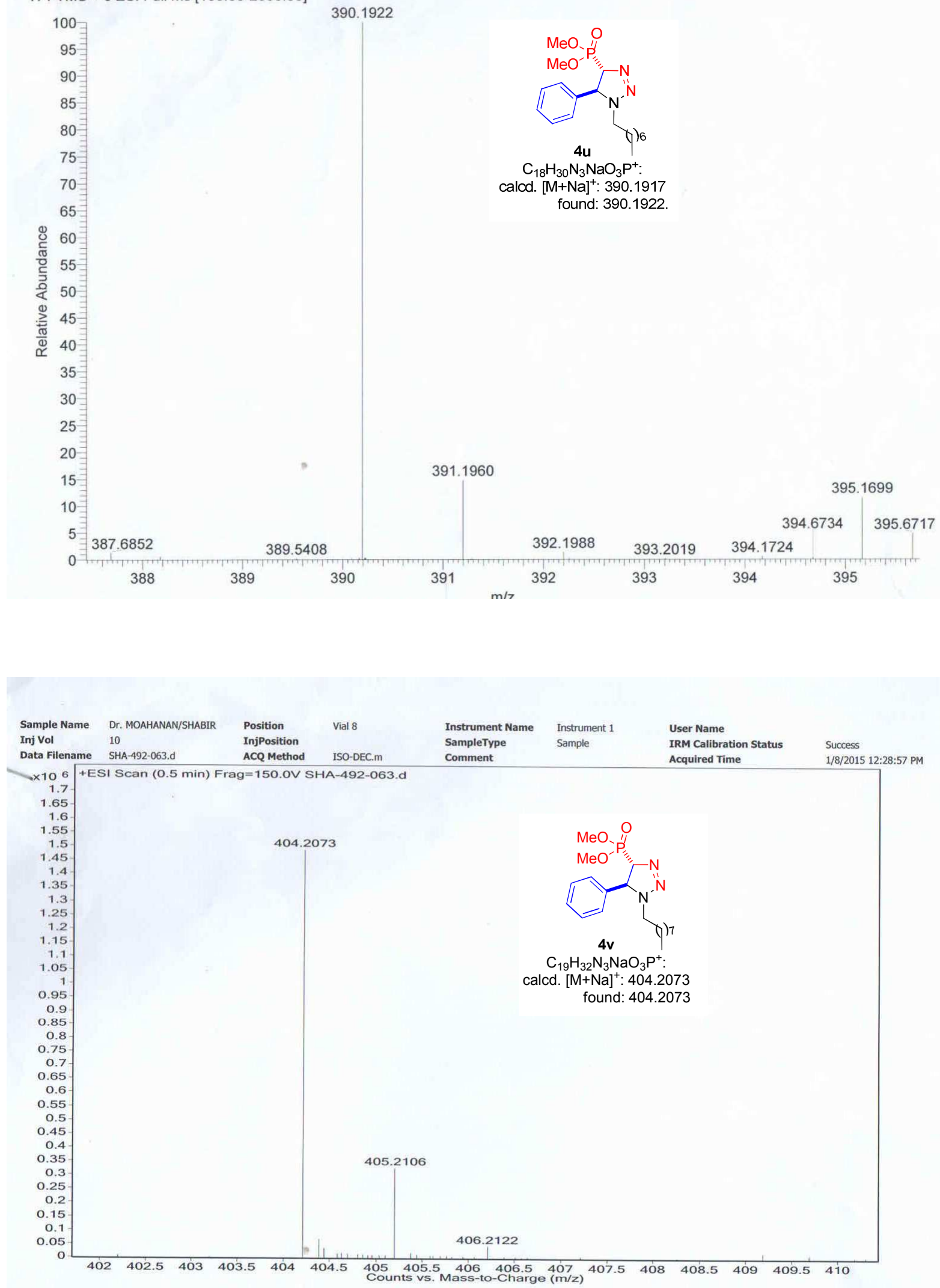

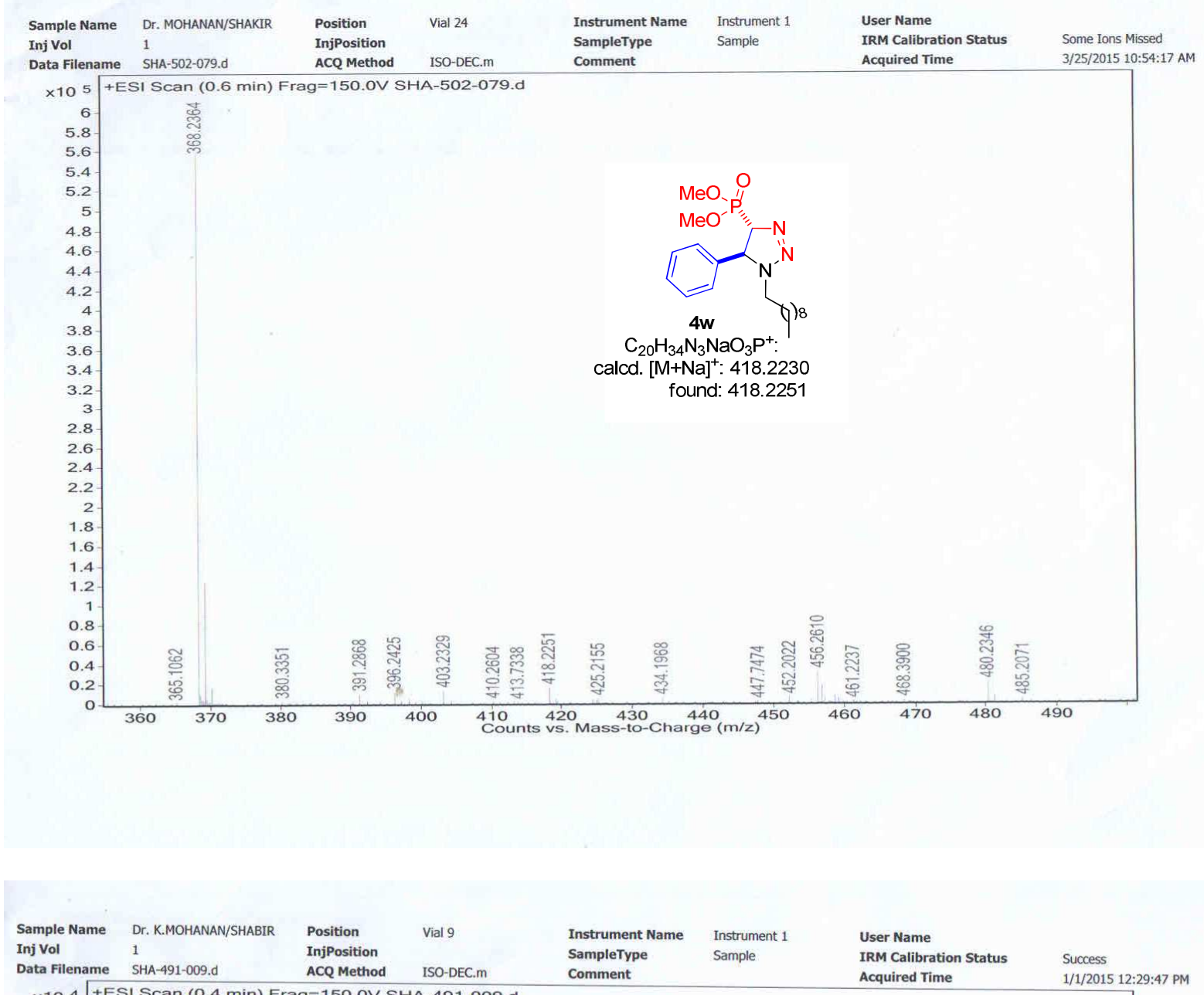
$\begin{array}{llll}\text { Acquired Time } & \text { Comment } & \text { ACQ Method } & \text { ISO-DEC.m }\end{array}$

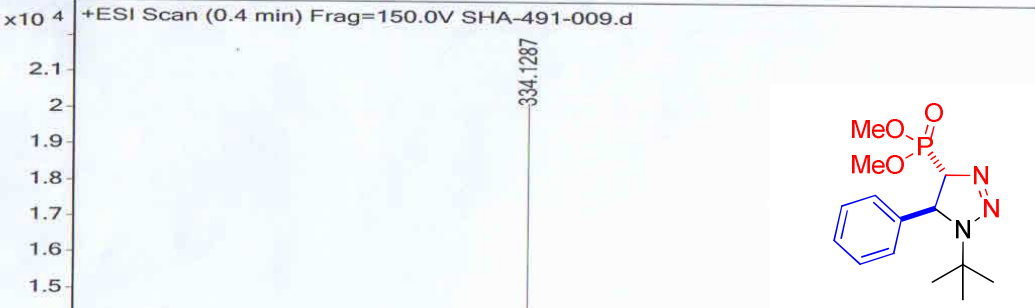

$4 \mathrm{x}$

$\mathrm{C}_{14} \mathrm{H}_{22} \mathrm{~N}_{3} \mathrm{NaO}_{3} \mathrm{P}^{+}$

calcd. [M+Na] : 334.1296

found: 334.1287 


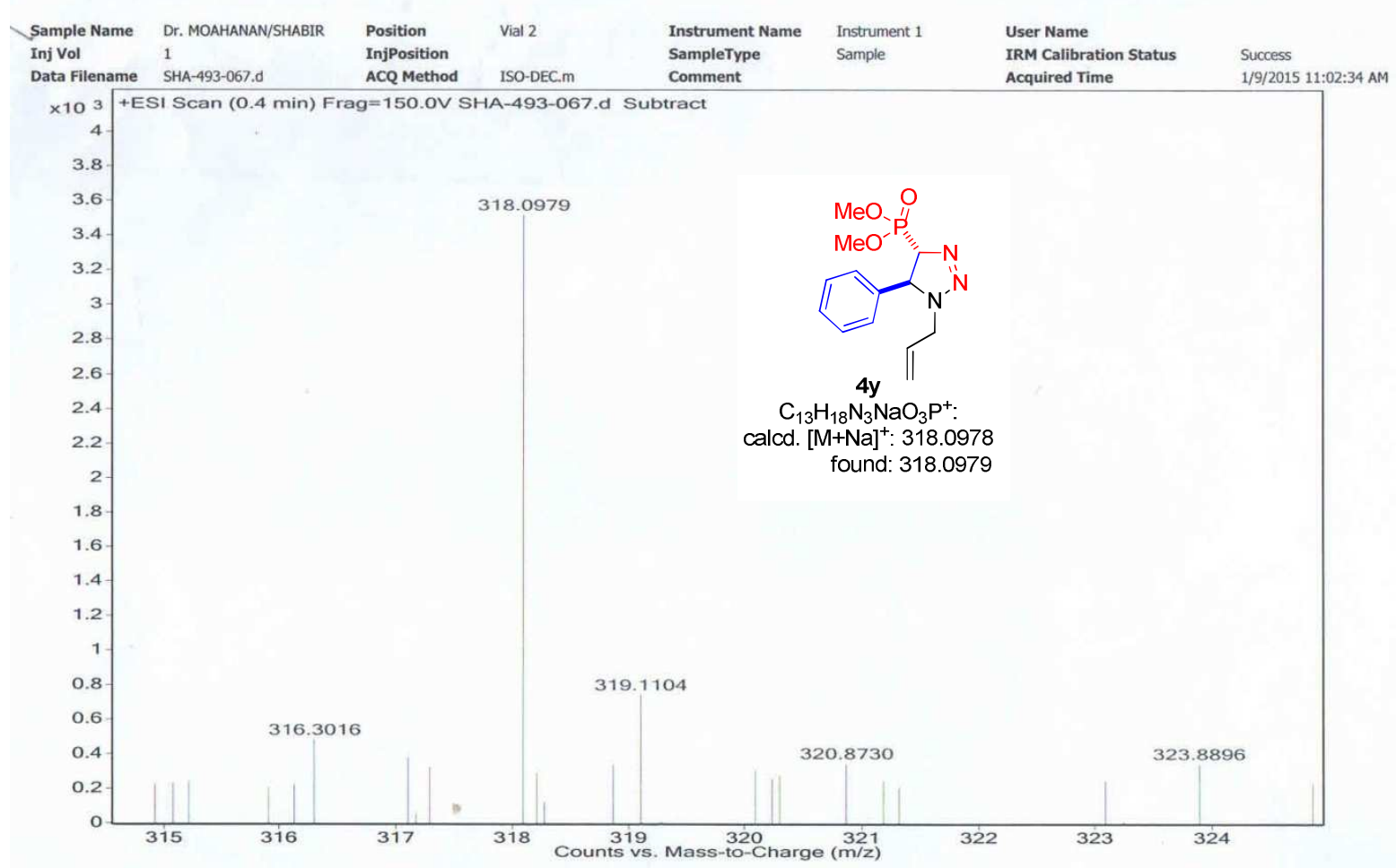

D:INTERNALS12015IDECITJR473

12/22/2015 4:08:22 PM

Dr. T.J. REDDY

TJR473 \#4-7 RT: 0.09-0.19 AV: 4 SB: 28 0.29-0.59, 0.73-1.31 NL: 2.91E5

T: FTMS + c ESI Full ms [150.00-1500.00]

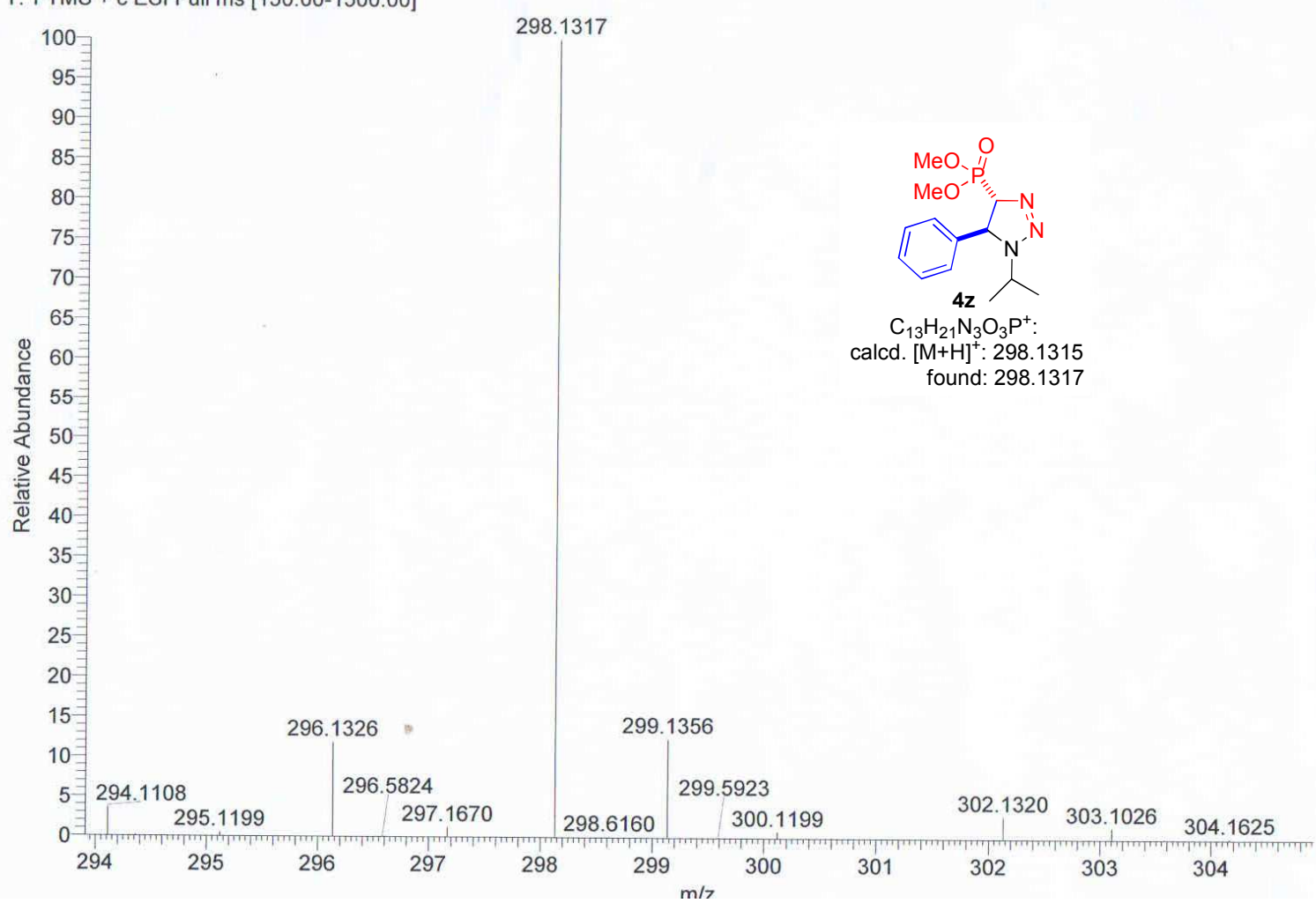




\begin{tabular}{|c|c|c|c|c|c|c|c|}
\hline Sample Name & Dr.MOHANAN/SHAKIR & Position & Vial 3 & Instrument Name & Instrument 1 & User Name & \\
\hline
\end{tabular}

$\times 104$ +ESI Scan (0.2-0.8 min, 38 Scans) Frag=150.0V SHA-674_155.d Subtract Acquired Time

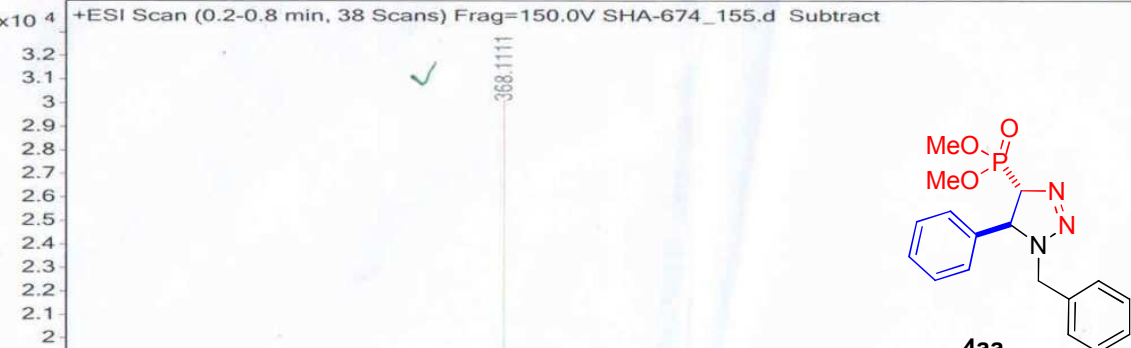

4 aa

$\mathrm{C}_{17} \mathrm{H}_{20} \mathrm{~N}_{3} \mathrm{NaO}_{3} \mathrm{P}^{+}$

calcd. $[\mathrm{M}+\mathrm{Na}]^{+}: 368.1134$

found: 368.1111

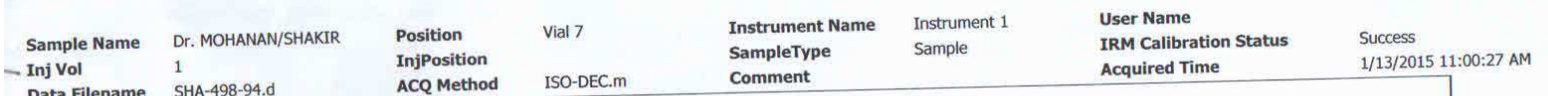

Data Filename SHA-498-94.d $\mathrm{min})$ Frag $=150$.OV SHA-498-94.d Subtract

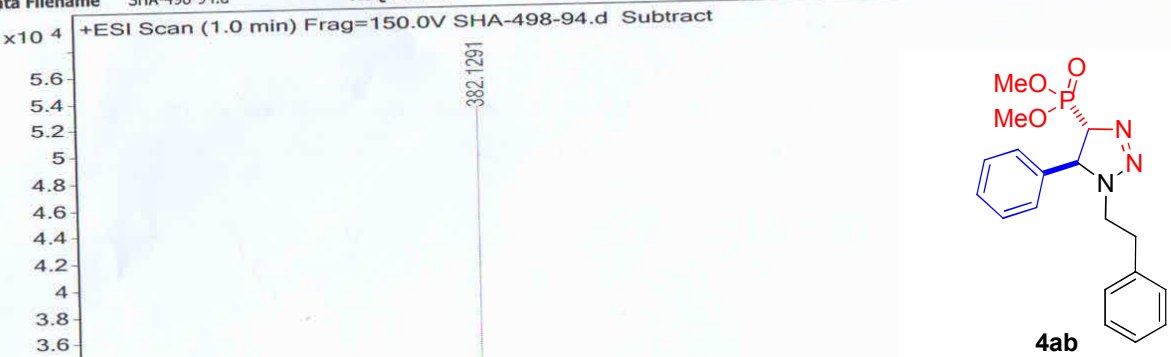

$\mathrm{C}_{18} \mathrm{H}_{22} \mathrm{~N}_{3} \mathrm{NaO}_{3} \mathrm{P}^{+}$.

calcd. [M+Na] $]^{+}: 382.1291$

found: 382.1291

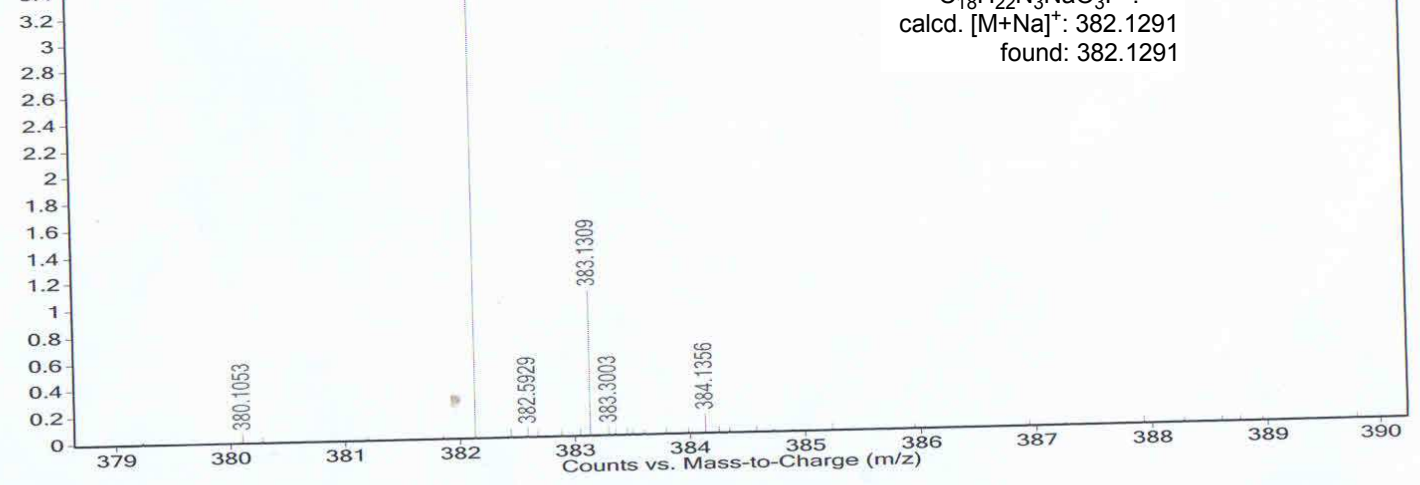


SHA-455\#10 RT: 0.13 AV: 1 SB: 5 0.01-0.06 NL: $1.63 E 7$

T: FTMS + c ESI Full ms [100.00-1200.00]
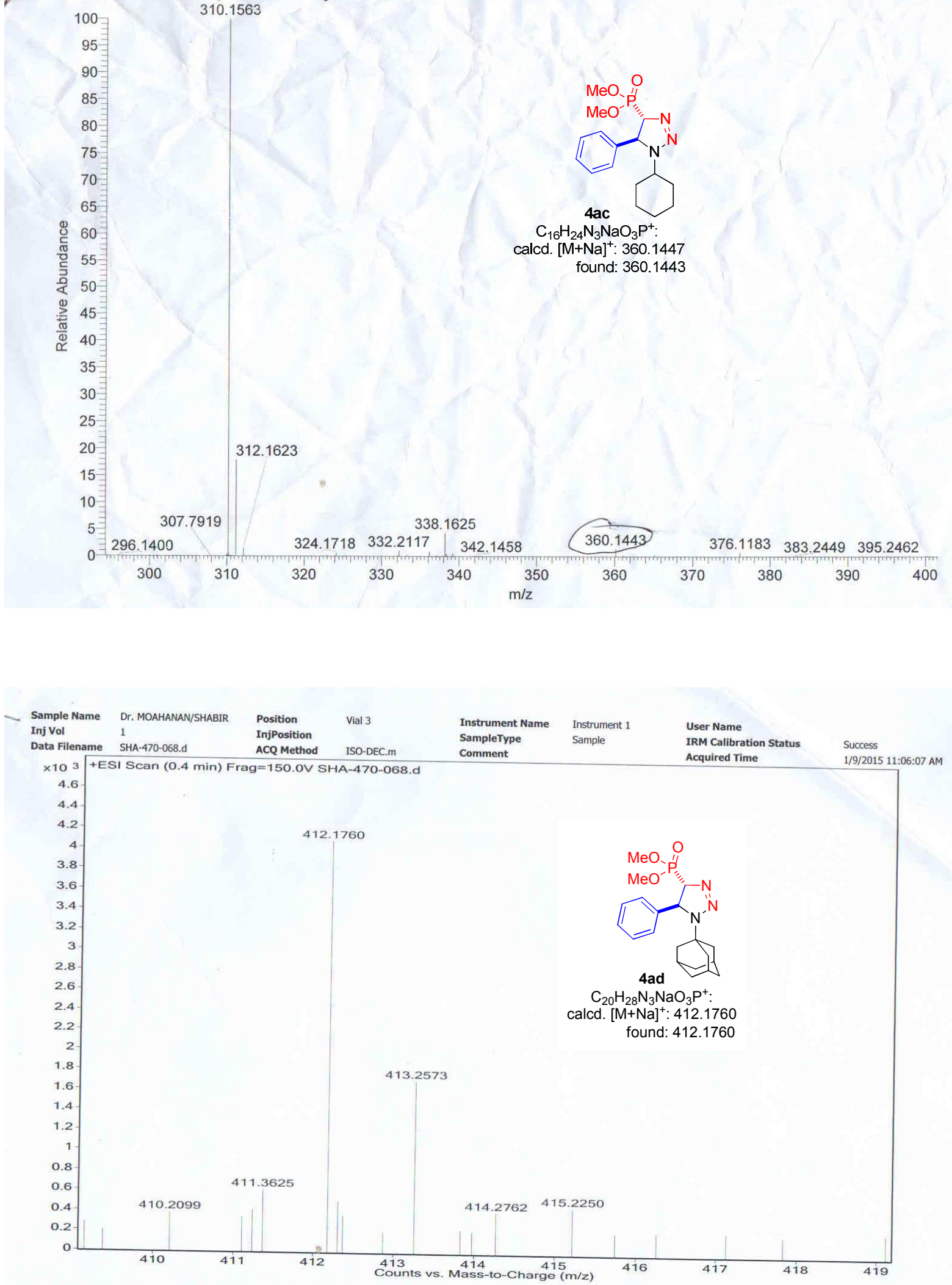


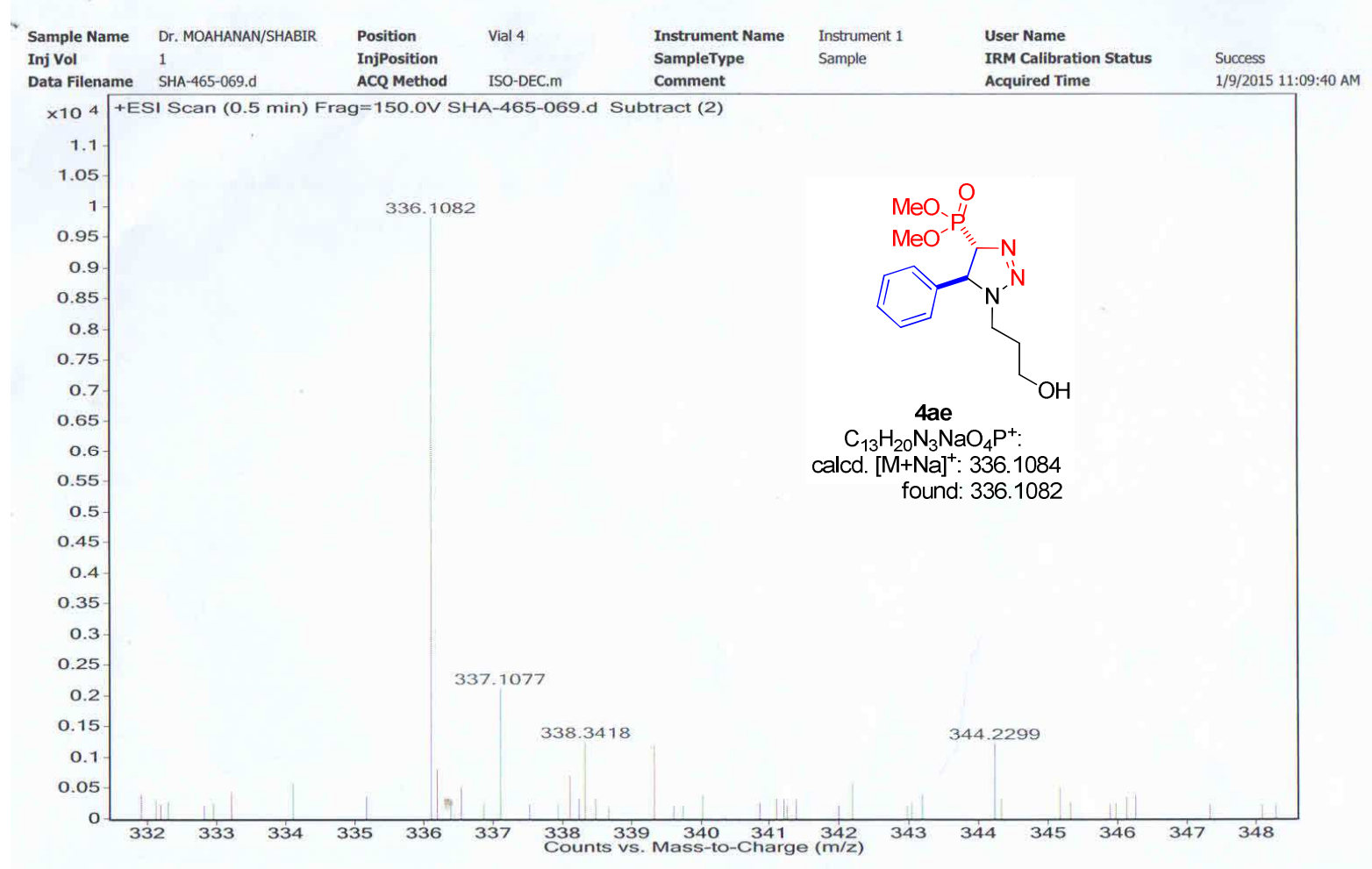

D:INTERNALSL2015IDECITJR506R

12/22/2015 4:32:49 PM

Dr. T.J. REDDY

TJR506R \#3-7 RT: 0.06-0.19 AV: 5 NL: $2.89 E 4$

T: FTMS + c ESI Full ms [150.00-1500.00]

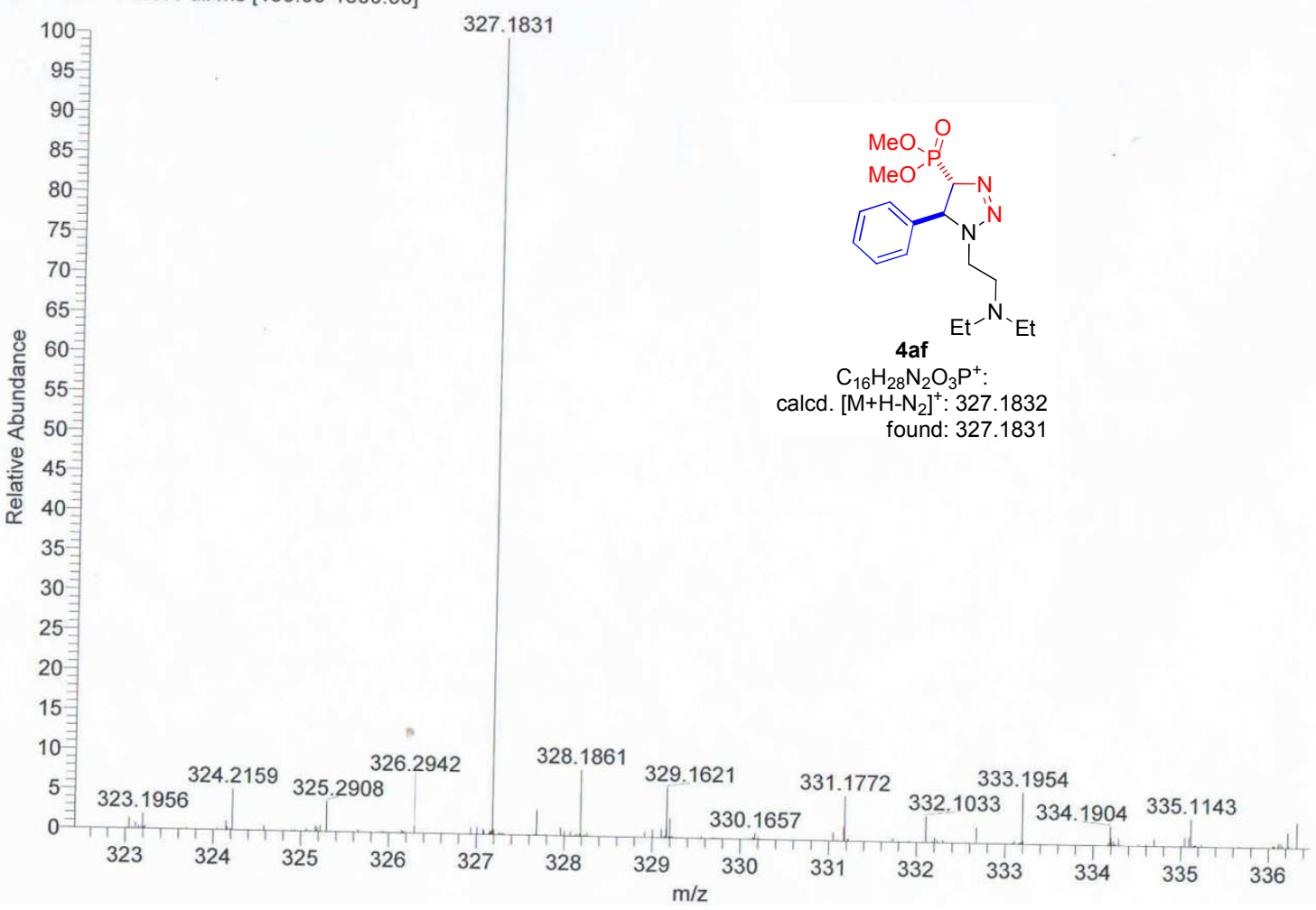




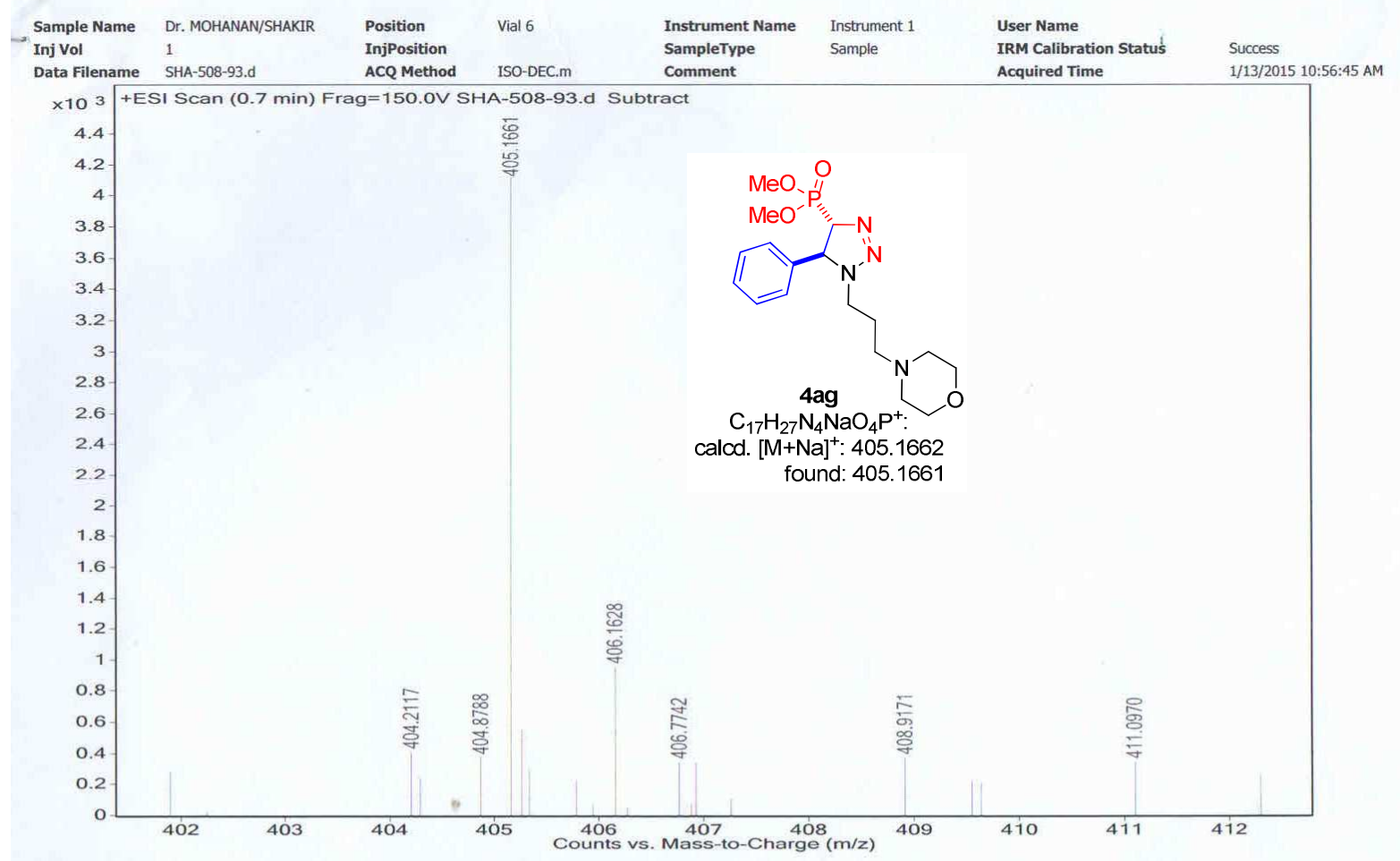

\begin{tabular}{|c|c|c|c|c|c|c|c|}
\hline Sample Name & Dr. MOHANAN/SHAKIR & Position & Vial 5 & Instrument Name & Instrument 1 & User Name & \\
\hline Data Filename & SHA-513-92.d & ACQ Method & ISO-DEC.m & Comment & & Acquired Time & 1/13/2015 10:53:09 AM \\
\hline
\end{tabular}

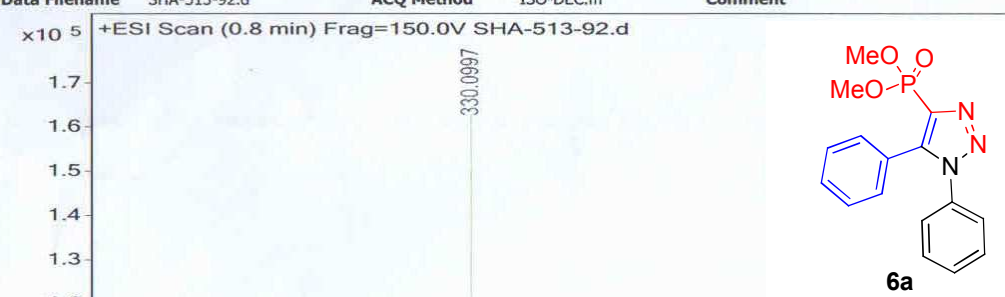

$\mathrm{C}_{16} \mathrm{H}_{17} \mathrm{~N}_{3} \mathrm{O}_{3} \mathrm{P}^{+}$:

calcd. $[\mathrm{M}+\mathrm{H}]^{+}: 330.1002$

found: 330.0997 


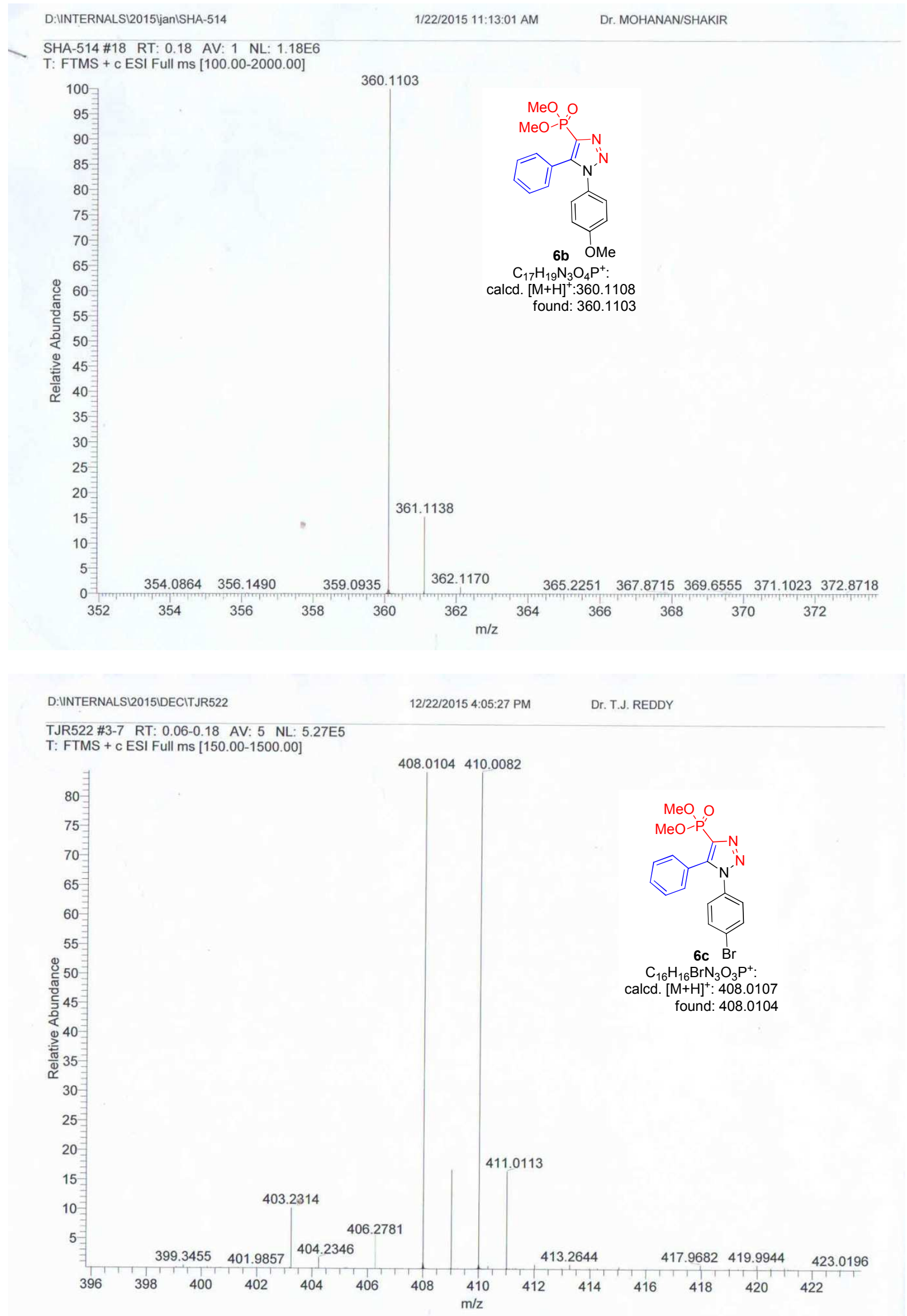



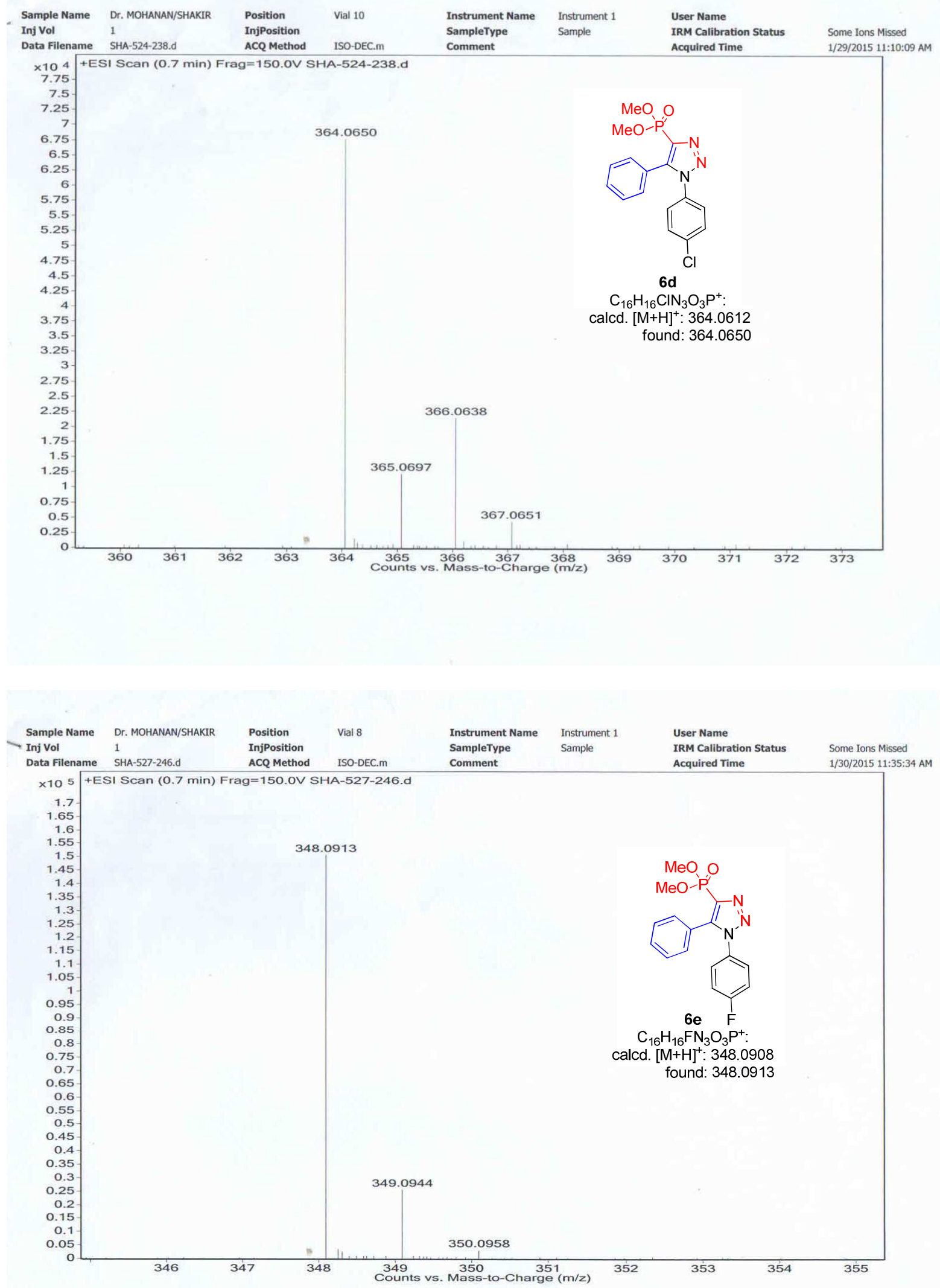


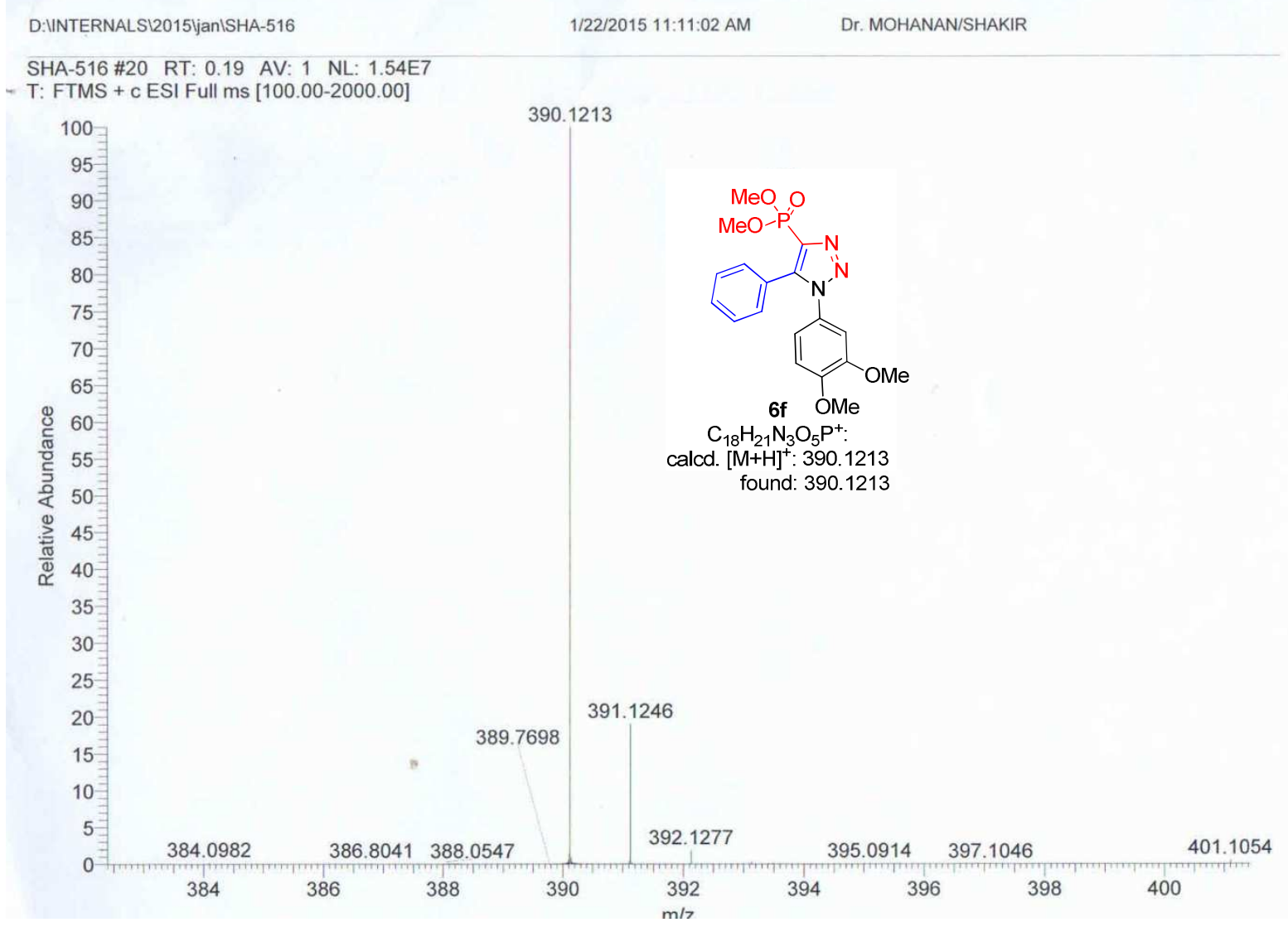

D:IINTERNALSL2015ljanISHA-517

Dr. MOHANAN/SHAKIR

SHA-517 \#26 RT: 0.24 AV: 1 NL: 2.73E6

T: FTMS + c ESI Full ms [100.00-2000.00]

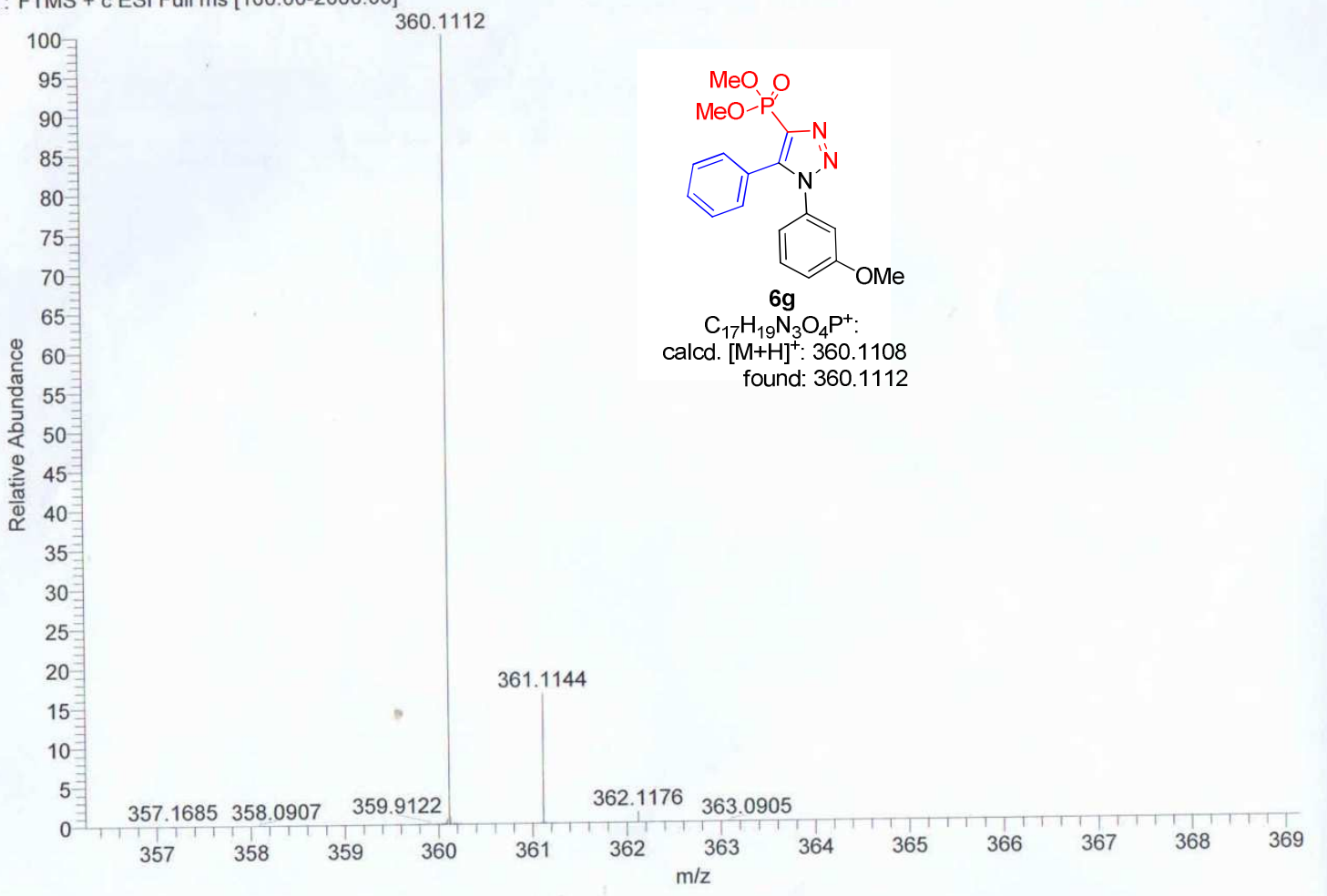




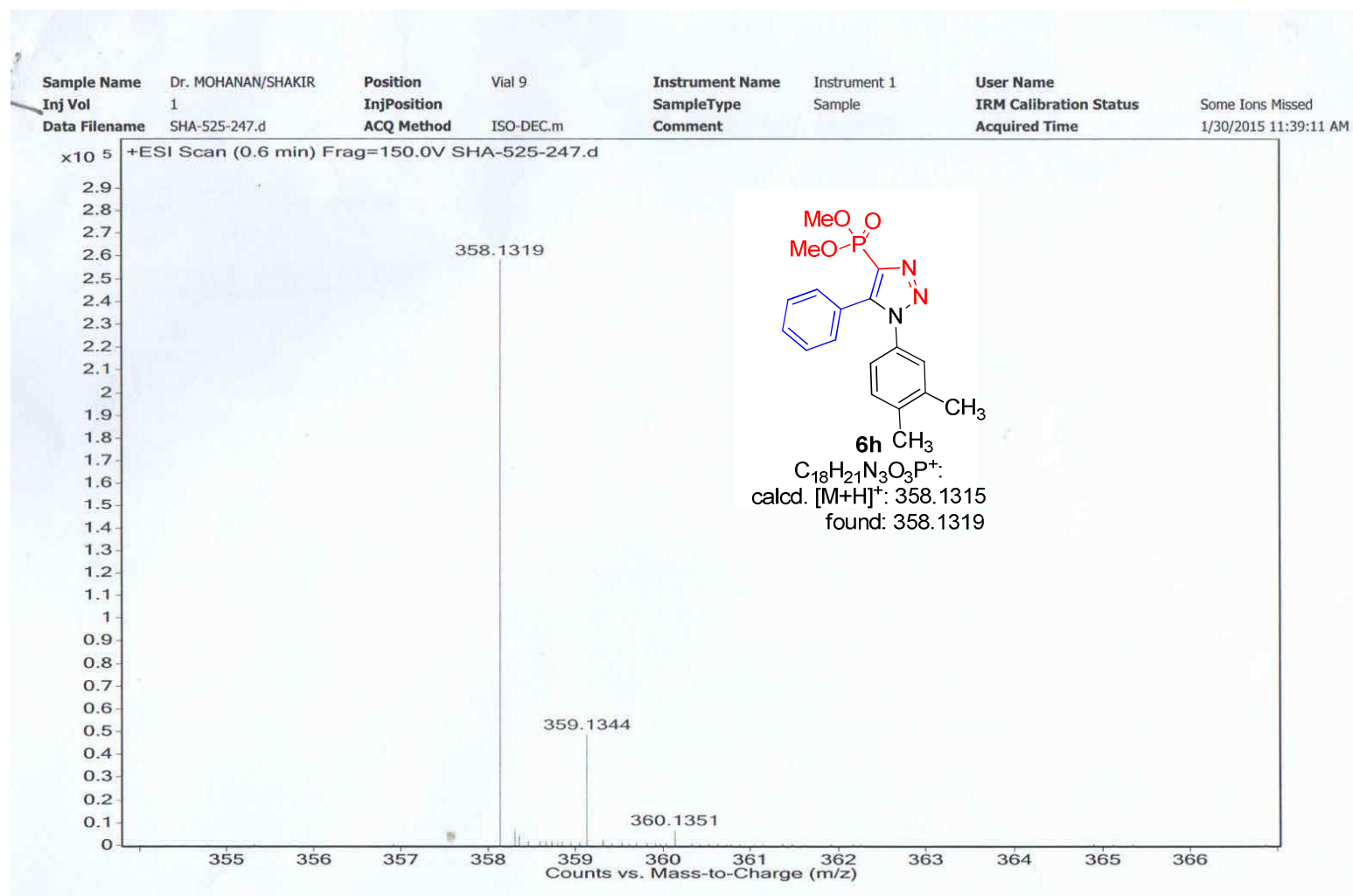

D:INTERNALSI...ISHA-520_150129120816

1/29/2015 12:08:16 PM

Dr. MOHANAN/SHAKIR

SHA-520_150129120816 \#34 RT: 0.40 AV: 1 NL: $1.02 E 6$

T: FTMS + c ESI Full ms [100.00-2000.00]

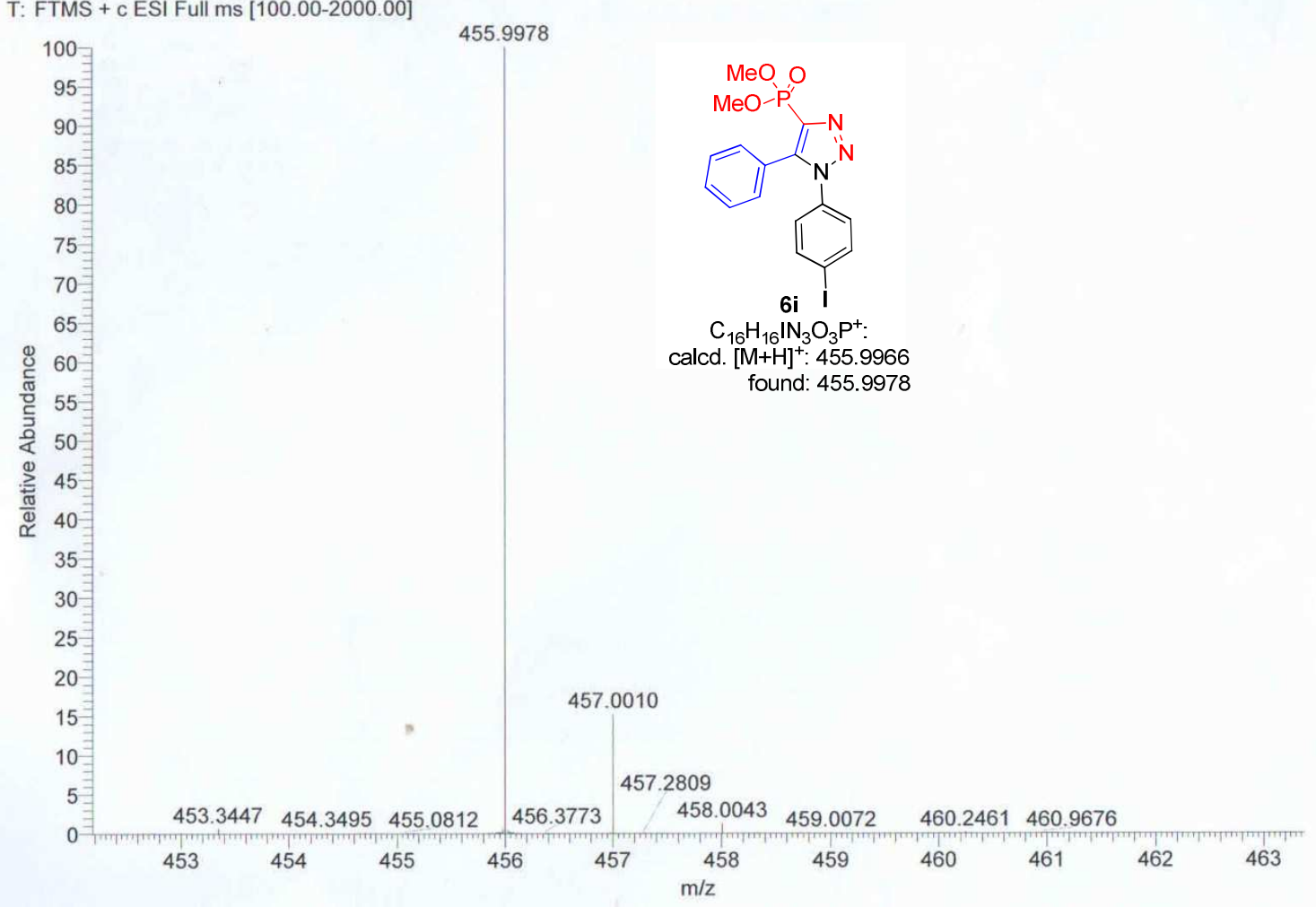


tjr-528R \#3-8 RT: 0.06-0.21 AV: 6 NL: 1.14E5

T: FTMS + c ESI Full ms [150.00-1500.00]
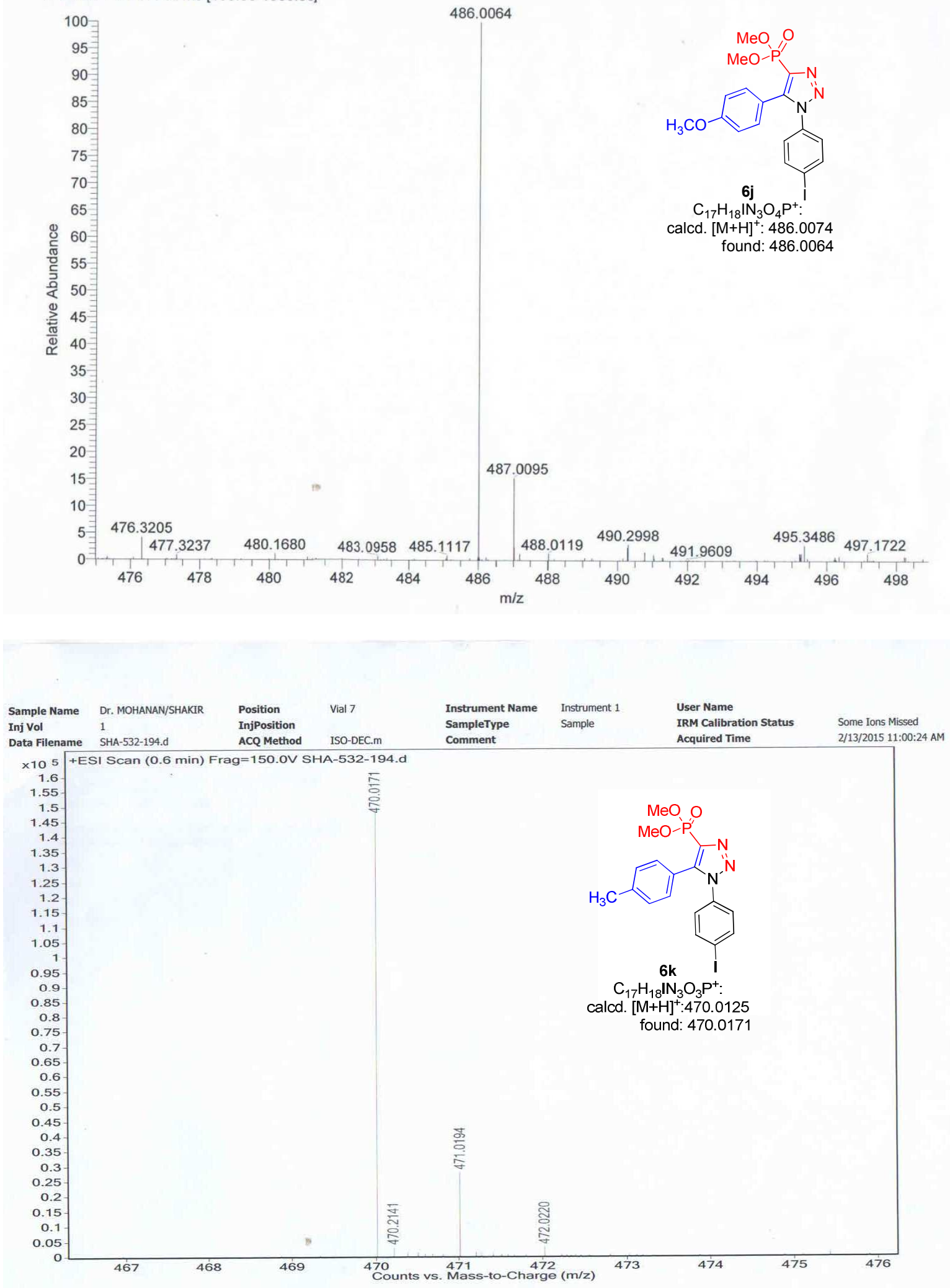


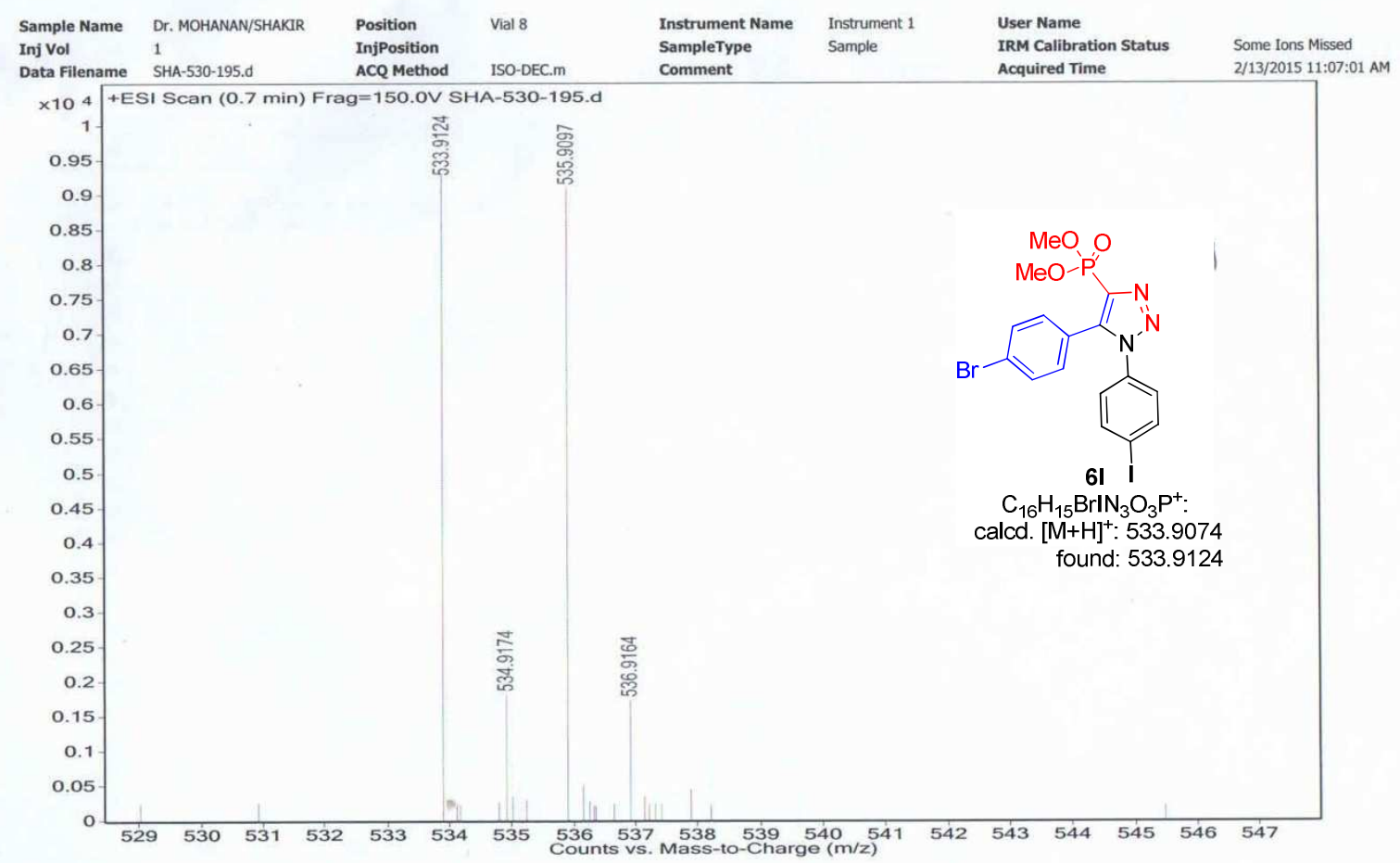

D:INTERNALSL2015IDECITJR-535

12/22/2015 3:56:35 PM

Dr. T.J. REDDY

TJR-535 \#2-7 RT: 0.03-0.18 AV: 6 SB: 28 0.28-0.59, 0.73-1.30 NL: $6.37 E 5$ T: FTMS + c ESI Full ms [150.00-1500.00]

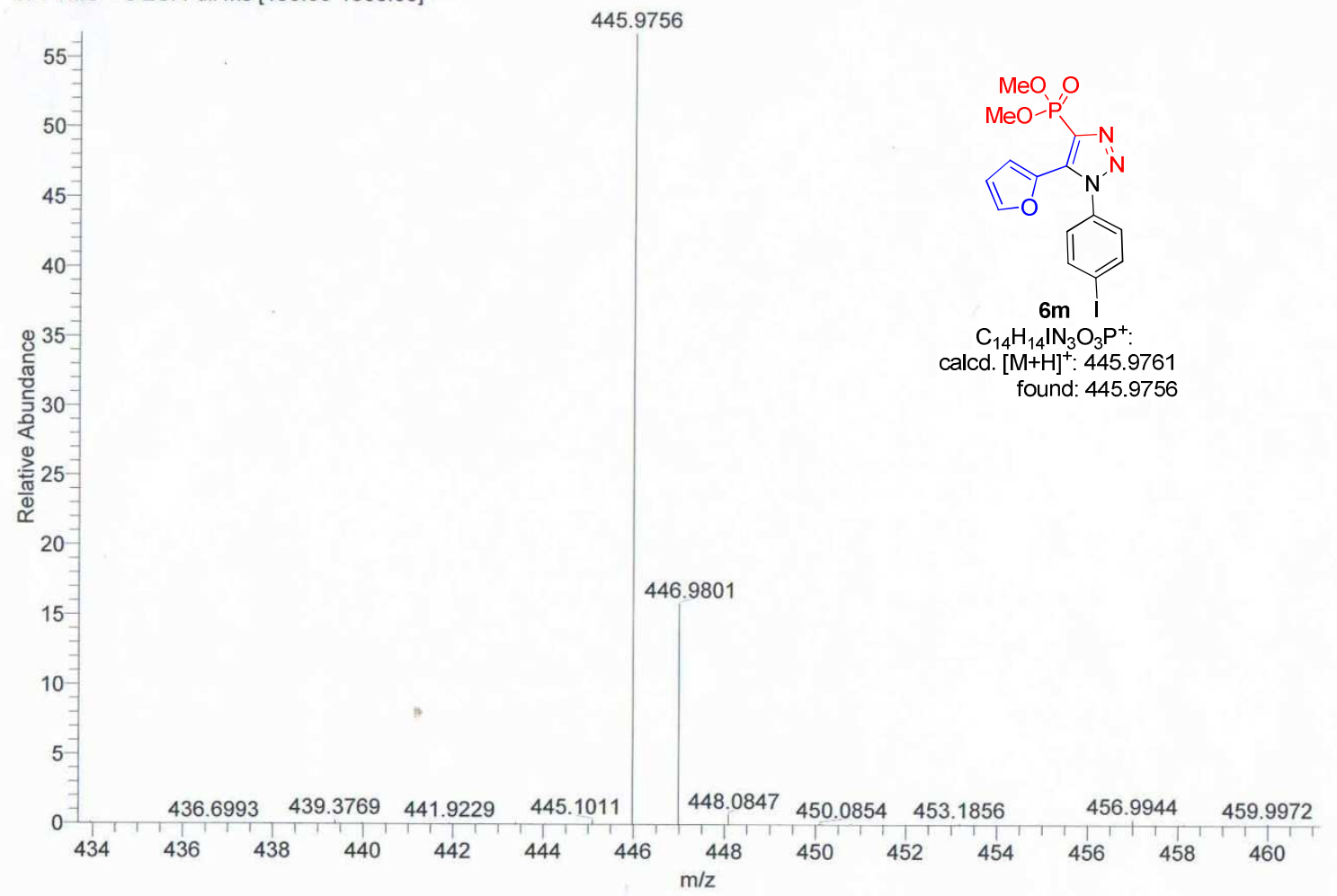

